\title{
Family-based prevention programmes for alcohol use in young people (Review)
}

Gilligan C, Wolfenden L, Foxcroft DR, Williams AJ, Kingsland M, Hodder RK, Stockings E, McFadyen TR, Tindall J, Sherker S, Rae J, Wiggers J

Gilligan C, Wolfenden L, Foxcroft DR, Williams AJ, Kingsland M, Hodder RK, Stockings E, McFadyen TR, Tindall J, Sherker S, Rae J, Wiggers J.

Family-based prevention programmes for alcohol use in young people.

Cochrane Database of Systematic Reviews 2019, Issue 3. Art. No.: CD012287.

DOI: 10.1002/14651858.CD012287.pub2. 
TABLE OF CONTENTS

HEADER

ABSTRACT

PLAIN LANGUAGE SUMMARY

SUMMARY OF FINDINGS

BACKGROUND

OBJECTIVES

METHODS

RESULTS

Figure 1.

Figure 2.

Figure 3.

DISCUSSION

Figure 4.

Figure 5.

Figure 6.

AUTHORS' CONCLUSIONS

ACKNOWLEDGEMENTS

REFERENCES

CHARACTERISTICS OF STUDIES

DATA AND ANALYSES

Analysis 1.1. Comparison 1 Any parent/family intervention vs no intervention/standard care, Outcome 1 Alcohol use_Prevalence.

Analysis 1.2. Comparison 1 Any parent/family intervention vs no intervention/standard care, Outcome 2 Alcohol use_Frequency.

Analysis 1.3. Comparison 1 Any parent/family intervention vs no intervention/standard care, Outcome 3 Alcohol use_Volume.

Analysis 1.4. Comparison 1 Any parent/family intervention vs no intervention/standard care, Outcome 4 Sensitivity_RoB_Alcohol use_Prevalence.

Analysis 1.5. Comparison 1 Any parent/family intervention vs no intervention/standard care, Outcome 5 Subgroup_universal_Alcohol use_Prevalence.

Analysis 1.6. Comparison 1 Any parent/family intervention vs no intervention/standard care, Outcome 6 Subgroup_selective/ indicated_Alcohol use_Prevalence.

Analysis 1.7. Comparison 1 Any parent/family intervention vs no intervention/standard care, Outcome 7 Subgroup_low intensity_Alcohol use_Prevalence.

Analysis 1.8. Comparison 1 Any parent/family intervention vs no intervention/standard care, Outcome 8 Subgroup_>12 months_Alcohol use_Prevalence.

Analysis 1.9. Comparison 1 Any parent/family intervention vs no intervention/standard care, Outcome 9 Subgroup_ethnicity minority_Alcohol use_Prevalence.

Analysis 1.10. Comparison 1 Any parent/family intervention vs no intervention/standard care, Outcome 10 Subgroup_ethnicity majority/caucasian_Alcohol use_Prevalence.

Analysis 1.11. Comparison 1 Any parent/family intervention vs no intervention/standard care, Outcome 11 Subgroup_no weekly or heavy drinking_Alcohol use_Prevalence.

Analysis 1.12. Comparison 1 Any parent/family intervention vs no intervention/standard care, Outcome 12 Sensitivity_RoB_Alcohol use_Frequency.

Analysis 1.13. Comparison 1 Any parent/family intervention vs no intervention/standard care, Outcome 13 Subgroup_universal_Alcohol use_Frequency.

Analysis 1.14.Comparison 1 Any parent/family intervention vs no intervention/standard care, Outcome 14 Subgroup_selective/ indicated_Alcohol use_Frequency.

Analysis 1.15. Comparison 1 Any parent/family intervention vs no intervention/standard care, Outcome 15 Subgroup_>12 months_Alcohol use_Frequency.

Analysis 1.16. Comparison 1 Any parent/family intervention vs no intervention/standard care, Outcome 16 Subgroup_ethnicity minority_Alcohol use_Frequency.

Analysis 1.17. Comparison 1 Any parent/family intervention vs no intervention/standard care, Outcome 17 Subgroup_ethnicity majority/caucasian_Alcohol use_Frequency. 
Analysis 1.18. Comparison 1 Any parent/family intervention vs no intervention/standard care, Outcome 18 Subgroup_no binge_Alcohol use_Frequency.

Analysis 1.19. Comparison 1 Any parent/family intervention vs no intervention/standard care, Outcome 19 Sensitivity_RoB_Alcohol use_Volume.

Analysis 1.20. Comparison 1 Any parent/family intervention vs no intervention/standard care, Outcome 20 Subgroup_universal_Alcohol use_Volume.

Analysis 1.21. Comparison 1 Any parent/family intervention vs no intervention/standard care, Outcome 21 Subgroup_selective/ indicated_Alcohol use_Volume.

Analysis 1.22. Comparison 1 Any parent/family intervention vs no intervention/standard care, Outcome 22 Subgroup_>12 months_Alcohol use_Volume.

Analysis 1.23. Comparison 1 Any parent/family intervention vs no intervention/standard care, Outcome 23 Subgroup_ethnicity_minority_Alcohol use_Volume.

Analysis 1.24. Comparison 1 Any parent/family intervention vs no intervention/standard care, Outcome 24 Subgroup_ethnicity majority/caucasian_Alcohol use_Volume.

Analysis 1.25. Comparison 1 Any parent/family intervention vs no intervention/standard care, Outcome 25 Subgroup_female only_Alcohol use_Volume.

Analysis 2.1. Comparison 2 Family-based and adolescent intervention vs intervention with young people alone, Outcome 1 Alcohol use_prevalence.

Analysis 2.2. Comparison 2 Family-based and adolescent intervention vs intervention with young people alone, Outcome 2 Alcohol use_frequency.

Analysis 2.3. Comparison 2 Family-based and adolescent intervention vs intervention with young people alone, Outcome 3 Sensitivity_RoB_Alcohol use_prevalence.

Analysis 2.4. Comparison 2 Family-based and adolescent intervention vs intervention with young people alone, Outcome 4 Subgroup_universal_Alcohol use_prevalence.

Analysis 2.5. Comparison 2 Family-based and adolescent intervention vs intervention with young people alone, Outcome 5 Sensitivity_RoB_Alcohol use_frequency.

Analysis 2.6. Comparison 2 Family-based and adolescent intervention vs intervention with young people alone, Outcome 6 Subgroup_universal_Alcohol use_frequency.

Analysis 2.7. Comparison 2 Family-based and adolescent intervention vs intervention with young people alone, Outcome 7 Subgroup_indicated_Alcohol use_frequency. 
[Intervention Review]

\section{Family-based prevention programmes for alcohol use in young people}

Conor Gilligan ${ }^{1}$, Luke Wolfenden², David R Foxcroft ${ }^{3}$, Amanda J Williams ${ }^{4}$, Melanie Kingsland², Rebecca K Hodder 4 , Emily Stockings ${ }^{5}$, Tameka-Rae McFadyen², Jenny Tindall4, Shauna Sherker2,6, Julie Rae6, John Wiggers 4

${ }^{1}$ School of Medicine and Public Health, University of Newcastle, Hunter Medical Research Institute, Callaghan, Australia. ${ }^{2}$ School of Medicine and Public Health, University of Newcastle, Callaghan, Australia. ${ }^{3}$ Department of Psychology, Social Work and Public Health, Oxford Brookes University, Oxford, UK. 4Hunter New England Population Health, Hunter New England Local Health District, Wallsend, Australia. ${ }^{5}$ National Drug and Alcohol Research Centre (NDARC), University of New South Wales, Sydney, Australia. ${ }^{6}$ Program Development and Evaluation, Alcohol and Drug Foundation, Melbourne, Australia

Contact address: Conor Gilligan, School of Medicine and Public Health, University of Newcastle, Hunter Medical Research Institute, Callaghan, NSW, 2308, Australia. Conor.Gilligan@newcastle.edu.au.

Editorial group: Cochrane Drugs and Alcohol Group

Publication status and date: New, published in Issue 3, 2019.

Citation: Gilligan C, Wolfenden L, Foxcroft DR, Williams AJ, Kingsland M, Hodder RK, Stockings E, McFadyen TR, Tindall J, Sherker S, Rae J, Wiggers J. Family-based prevention programmes for alcohol use in young people. Cochrane Database of Systematic Reviews 2019, Issue 3. Art. No.: CD012287. DOI: 10.1002/14651858.CD012287.pub2.

Copyright @ 2019 The Cochrane Collaboration. Published by John Wiley \& Sons, Ltd.

\section{A B S T R A C T}

\section{Background}

Alcohol use in young people is a risk factor for a range of short- and long-term harms and is a cause of concern for health services, policymakers, youth workers, teachers, and parents.

\section{Objectives}

To assess the effectiveness of universal, selective, and indicated family-based prevention programmes in preventing alcohol use or problem drinking in school-aged children (up to 18 years of age).

Specifically, on these outcomes, the review aimed:

- to assess the effectiveness of universal family-based prevention programmes for all children up to 18 years ('universal interventions');

- to assess the effectiveness of selective family-based prevention programmes for children up to 18 years at elevated risk of alcohol use or problem drinking ('selective interventions'); and

- to assess the effectiveness of indicated family-based prevention programmes for children up to 18 years who are currently consuming alcohol, or who have initiated use or regular use ('indicated interventions').

\section{Search methods}

We identified relevant evidence from the Cochrane Central Register of Controlled Trials (CENTRAL), in the Cochrane Library, MEDLINE (Ovid 1966 to June 2018), Embase (1988 to June 2018), Education Resource Information Center (ERIC; EBSCOhost; 1966 to June 2018), PsycINFO (Ovid 1806 to June 2018), and Google Scholar. We also searched clinical trial registers and handsearched references of topicrelated systematic reviews and the included studies.

\section{Selection criteria}

We included randomised controlled trials (RCTs) and cluster RCTs (C-RCTs) involving the parents of school-aged children who were part of the general population with no known risk factors (universal interventions), were at elevated risk of alcohol use or problem drinking (selective interventions), or were already consuming alcohol (indicated interventions). Psychosocial or educational interventions involving 
parents with or without involvement of children were compared with no intervention, or with alternate (e.g. child only) interventions, allowing experimental isolation of parent components.

\section{Data collection and analysis}

We used standard methodological procedures expected by Cochrane.

\section{Main results}

We included 46 studies (39,822 participants), with 27 classified as universal, 12 as selective, and seven as indicated. We performed metaanalyses according to outcome, including studies reporting on the prevalence, frequency, or volume of alcohol use. The overall quality of evidence was low or very low, and there was high, unexplained heterogeneity.

Upon comparing any family intervention to no intervention/standard care, we found no intervention effect on the prevalence (standardised mean difference (SMD) $0.00,95 \%$ confidence interval $(\mathrm{Cl})-0.08$ to 0.08 ; studies $=12$; participants $=7490 ; I^{2}=57 \%$; low-quality evidence) or frequency (SMD - $0.31,95 \% \mathrm{Cl}-0.83$ to 0.21 ; studies $=8$; participants $=1835 ; \mathrm{I}^{2}=96 \%$; very low-quality evidence) of alcohol use in comparison with no intervention/standard care. The effect of any parent/family interventions on alcohol consumption volume compared with no intervention/standard care was very small (SMD $-0.14,95 \% \mathrm{Cl}-0.27$ to 0.00 ; studies $=5$; participants $=1825 ; \mathrm{I}^{2}=42 \%$; low-quality evidence).

When comparing parent/family and adolescent interventions versus interventions with young people alone, we found no difference in alcohol use prevalence (SMD $-0.39,95 \% \mathrm{Cl}-0.91$ to 0.14 ; studies $=4$; participants $=5640 ; I^{2}=99 \%$; very low-quality evidence) or frequency $\left(\mathrm{SMD}-0.16,95 \% \mathrm{Cl}-0.42\right.$ to 0.09 ; studies $=4$; participants $=915 ; \mathrm{I}^{2}=73 \%$; very low-quality evidence). For this comparison, no trials reporting on the volume of alcohol use could be pooled in meta-analysis.

In general, the results remained consistent in separate subgroup analyses of universal, selective, and indicated interventions. No adverse effects were reported.

\section{Authors' conclusions}

The results of this review indicate that there are no clear benefits of family-based programmes for alcohol use among young people. Patterns differ slightly across outcomes, but overall, the variation, heterogeneity, and number of analyses performed preclude any conclusions about intervention effects. Additional independent studies are required to strengthen the evidence and clarify the marginal effects observed.

\section{PLAIN LANGUAGE SUMMARY}

\section{Family-based prevention of youth alcohol use}

\section{Review question}

We reviewed the evidence about the effects of family- or parent-based programmes as a way of preventing or reducing alcohol use in school-aged children.

\section{Background}

Alcohol use puts young people at increased risk for a range of short- and long-term harms and is a cause of concern for health services, policy-makers, youth workers, teachers, and parents.

\section{Search date}

The evidence was current to June 2018.

\section{Study characteristics}

We found 46 randomised controlled trials (studies where participants were randomly allocated to one of two or more intervention or control groups) that compared family-based interventions versus no intervention or an adolescent component alone. We included studies targeting general populations of parents and children (universal interventions), those targeting parents of children at increased risk of alcohol use (selective interventions), and studies targeting parents of children already using alcohol (indicated interventions). We were interested in studies following participants up to four years post intervention.

Most studies were conducted in the United States or in European countries (the Netherlands, Sweden, Poland, and Germany). One study was conducted in India. Interventions were delivered in various settings including the child's school or family home and via the Internet or print material. Interventions varied in intensity, duration, and approach, but all targeted alcohol or other drug use by promoting positive parenting approaches or enhancing parent-child relationships. The interventions focused on communication, family dynamics, rule-setting, and risk management. 
The total number of participants in the included studies was 39,822, and the young people targeted ranged from 5 to 17 years of age. Participant ethnicity was mixed, with 12 studies targeting ethnic minority groups specifically.

\section{Key results}

Overall, we found no evidence for the effectiveness of family-based interventions on the prevalence, frequency, or volume of alcohol use among young people. Some analyses focusing on specific subgroups of studies (e.g. including only universal interventions, targeting ethnic minority groups) showed small intervention effects, but considering variation in results, variation between studies, and overall low quality of the evidence, we are uncertain whether these interventions have a positive effect on young people's alcohol consumption. Some studies reported positive intervention effects on secondary outcomes (parental supply of alcohol, family involvement, alcohol misuse, and alcohol dependence) but with small numbers; these studies could not be pooled, so the evidence is insufficient. No adverse effects were reported.

\section{Quality of evidence}

Overall, only very low- or low-quality evidence shows the small effects found in this review. Many of the studies did not adequately describe how families/young people/parents were allocated to the study groups, or how they concealed the group allocation from participants and personnel. We downgraded the quality of evidence due to the heterogeneity (variability) between studies and imprecision (variation) in results. These problems with study quality could result in inflated estimates of intervention effects, so we cannot rule out the possibility that slight effects observed in this review may be overstated.

The US National Institutes of Health (NIH) and the National Institutes of Alcohol Abuse and Alcoholism (NIAAA), Drug Abuse (NIDA), and Mental Health provided funding for over half $(28 / 46)$ of the studies included in this review. Three studies provided no information about funding, and only 13 papers had a clear conflict of interest statement. 
SUMMARY OF FINDINGS

\section{Summary of findings for the main comparison. Family/parent interventions compared with control for reducing alcohol consumption in adolescents}

\section{Family/parent interventions compared with no intervention/standard care for prevalence of adolescent alcohol consumption}

Patient or population: parents/children

Settings: recruitment through schools $(n=11)$, communities $(n=6)$, paediatric emergency departments $(n=2)$, other health clinics ( $n=2)$; referral by schools, the justice system, therapists, physicians, or parents $(n=1)$; street-based recruitment $(n=1)$ or random digit dialling $(n=1)$; and delivery via resources sent home $(n=3)$; face-face in schools, homes, or community venues $(n=14)$; or via the Internet or computer $(n=2)$

Intervention: parent interventions (positive parenting and communication and counselling sessions)

Comparison: no intervention

\begin{tabular}{|c|c|c|c|c|c|c|}
\hline \multirow[t]{3}{*}{ Outcomes } & \multicolumn{2}{|c|}{ Illustrative comparative risks* $(95 \% \mathrm{Cl})$} & \multirow{3}{*}{$\begin{array}{l}\text { Relative ef- } \\
\text { fect } \\
(95 \% \mathrm{Cl})\end{array}$} & \multirow{3}{*}{$\begin{array}{l}\text { No. of partic- } \\
\text { ipants } \\
\text { (studies) }\end{array}$} & \multirow{3}{*}{$\begin{array}{l}\text { Quality of the } \\
\text { evidence } \\
\text { (GRADE) }\end{array}$} & \multirow[t]{3}{*}{ Comments } \\
\hline & Assumed risk & Corresponding risk & & & & \\
\hline & $\begin{array}{l}\text { Risk with no interven- } \\
\text { tion }\end{array}$ & Risk with parent intervention & & & & \\
\hline $\begin{array}{l}\text { Alcohol use preva- } \\
\text { lence } \\
\text { Up to } 4 \text { years post } \\
\text { intervention im- } \\
\text { pact of family/par- } \\
\text { ent interventions } \\
\text { compared to con- } \\
\text { trol on the preva- } \\
\text { lence of alcohol } \\
\text { consumption or } \\
\text { drunkenness }\end{array}$ & $\begin{array}{l}\text { Mean prevalence of life- } \\
\text { time alcohol use was } \\
85 \% a\end{array}$ & $\begin{array}{l}\text { Mean prevalence of lifetime alcohol } \\
\text { use in intervention groups was zero } \\
\text { ( } 0.16 \text { lower to } 0.16 \text { higher) }\end{array}$ & & $\begin{array}{l}7490 \\
\text { (12 RCTs) }\end{array}$ & $\begin{array}{l}\oplus \oplus \ominus \ominus \\
\text { low } b\end{array}$ & $\begin{array}{l}\text { Scores estimated using a } \\
\text { standardised mean differ- } \\
\text { ence of } 0.00(95 \% \mathrm{Cl}-0.08 \\
\text { to } 0.08)\end{array}$ \\
\hline
\end{tabular}

pact of family/par-

ent interventions

compared to con-

trol on the frequen- 


\section{Alcohol use vol-}

ume

Up to 4 years post intervention im-

pact of family/par-

ent interventions

compared to con-

trol on the volume

of alcohol con-

sumption or drunk

enness

\section{Mean number of drinks}

in the last 30 days

among control groups

was $0.83 e$
Mean number of drinks in intervention

groups was 0.18 lower ( 0.34 lower to

0.00 higher)
1825 (5 RCTs)

$\oplus \oplus \ominus \ominus$

low $f$
Scores estimated using a standardised mean difference of $-0.14(95 \% \mathrm{Cl}-0.27$ to 0.00 )

Adverse events

No studies reported this outcome

*The basis for the assumed risk (e.g. the median control group risk across studies) is provided in footnotes. The corresponding risk (and its $95 \%$ confidence interval) is

based on the assumed risk in the comparison group and the relative effect of the intervention (and its $95 \% \mathrm{Cl}$ ).

$\mathrm{Cl}$ : confidence interval; RCT: randomised controlled trial.

GRADE Working Group grades of evidence.

High quality: further research is very unlikely to change our confidence in the estimate of effect.

Moderate quality: further research is likely to have an important impact on our confidence in the estimate of effect and may change the estimate.

Low quality: Further research is very likely to have an important impact on our confidence in the estimate of effect and is likely to change the estimate.

Very low quality: We are very uncertain about the estimate.

aWe have used results (mean scores and standard deviation) from Bauman 2002 to illustrate effect sizes in terms of the measures used in that study. This study was chosen for its use of common outcome measures and intervention approach, and low risk of bias.

${ }^{b}$ Downgraded one level due to high or unclear risk of bias ratings in several domains; downgraded one level due to moderate heterogeneity that was explained only in part in subgroup analysis.

cWe have used results (mean scores and standard deviation) from Winters 2012 to illustrate effect sizes in terms of the measures used in that study. This study was chosen for its use of common outcome measures and low risk of bias.

$d$ Downgraded one level due to high or unclear risk of bias ratings in several domains; downgraded two levels due to high heterogeneity that was not explained in subgroup analysis; downgraded one level due to imprecision of results, with a wide confidence interval (crosses -0.5 and crosses zero; therefore the true effect could be either a benefit or a harm). eWe have used results (mean scores and standard deviation) from Mason 2012 to illustrate effect sizes in terms of the measures used in that study. Although this study provides only small numbers and favours the control condition, it was chosen for the availability of mean and standard deviation derived from a common outcome measure and low risk of bias. fDowngraded one level due to high or unclear risk of bias ratings in several domains; downgraded one level due to a high probability of selective reporting bias, with the metaanalysis potentially not representative of available studies (only 5 out 9 included studies were included in meta-analysis). 


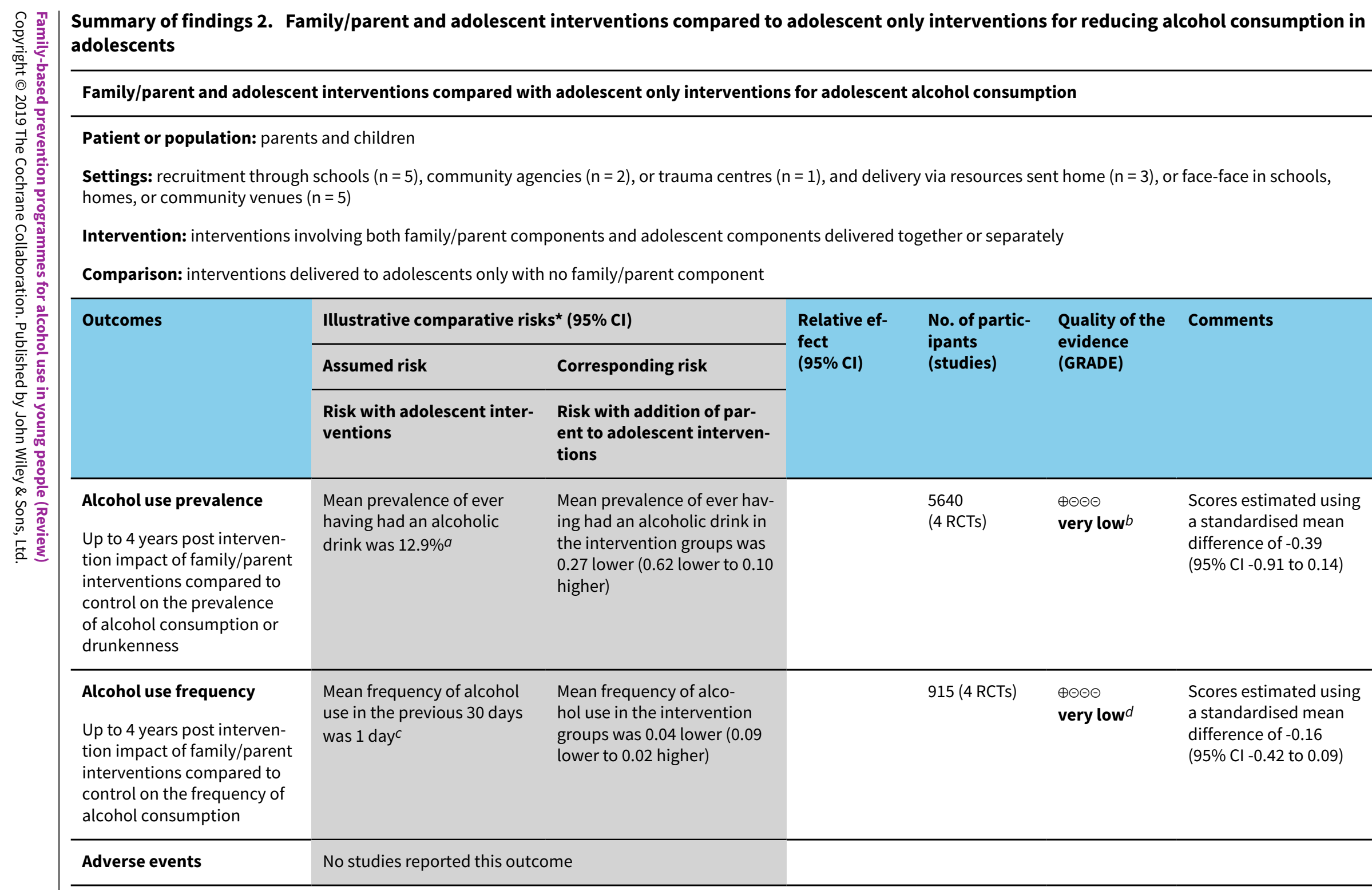

*The basis for the assumed risk (e.g. the median control group risk across studies) is provided in footnotes. The corresponding risk (and its $95 \%$ confidence interval) is based on the assumed risk in the comparison group and the relative effect of the intervention (and its $95 \% \mathrm{Cl}$ ).

Cl: confidence interval; RCT: randomised controlled trial. 
High quality: further research is very unlikely to change our confidence in the estimate of effect.

Moderate quality: further research is likely to have an important impact on our confidence in the estimate of effect and may change the estimate.

Low quality: further research is very likely to have an important impact on our confidence in the estimate of effect and is likely to change the estimate.

Very low quality: we are very uncertain about the estimate.

aWe have used results (mean scores and standard deviation 0.68) from Reddy 2002 to illustrate effect sizes in terms of the measures used in that study. This study was chosen for its use of a common outcome measure and low risk of bias.

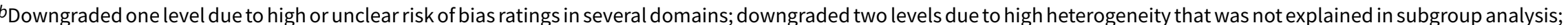
downgraded one level due to imprecision of results, with a wide confidence interval (crosses - 0.5 and crosses zero; therefore the true effect could be either a benefit or a harm). cWe have used results (mean scores and standard deviation) from Schinke 2004 to illustrate effect sizes in terms of the measures used in that study. This study was chosen for its use of a common outcome measure and low risk of bias.

$d$ Downgraded one level due to high or unclear risk of bias ratings in several domains; downgraded one level due to moderate heterogeneity that was explained only in part in subgroup analysis; downgraded one level due to imprecision of results, with a wide confidence interval (crosses - 0.5 and crosses zero; therefore the true effect could be either a benefit or a harm) and a relatively small sample size. 


\section{B A C K G R O U N D}

\section{Description of the condition}

Alcohol use ranks among the top three risk factors for the global burden of disease, accounting for $5.5 \%$ of disability-adjusted lifeyears (DALYs) globally (Lim 2012). A causal relationship has been established between alcohol and more than 200 chronic and acute diseases, as well as intentional and unintentional injuries (Rehm 2010). Overall, in 2010, alcohol-attributable injuries were responsible for $13.2 \%$ of all injury deaths and for $12.6 \%$ of all injury potential years of life lost (PYLL) (Rehm 2013). Young people contribute a high proportion of alcohol-related injuries and mortality from alcohol-attributable injury, with $11 \%$ of deaths among men aged 15 to 34 years, and 3.5\% of deaths among women aged 15 to 34 years in the European Union, being alcohol-related (Rehm 2012). In the European Union, road traffic accidents are the leading cause of death in children and young adults up to 29 years, and $33 \%$ of motor vehicle traffic injuries to males and $11 \%$ to females of all ages are due to alcohol (WHO 2012). Extensive evidence points to an association between early age of alcohol use initiation (and early intoxication) and an increased frequency of drinking, as well as increased risky drinking and alcohol-related harms later in adolescence and during adulthood (e.g. Bonomo 2004; DeWit 2000; Jackson 2015; Kuntsche 2013; Livingston 2008; Waller 2018).

Experimentation with risky behaviours typically begins in adolescence, as part of a natural 'coming of age' process (Room 2004). A dramatic increase in the use of alcohol is seen after the age of 12 , with rates gradually increasing throughout adolescence (Currie 2012). This pattern is common globally, with reports from 43 countries included in the Health Behaviour in School-Aged Children Project (Currie 2012), reports from the European Survey Project on Alcohol and Drugs (ESPAD; Hibell 2012), and results of national surveys conducted in Australia - White 2012 - and the United States (US) - Frieden 2014 - demonstrating these patterns. Any level of alcohol use is potentially harmful for young people, with evidence of an effect upon the developing brain (Bava 2010), along with a subsequent increase in risk for alcohol use disorders (Waller 2018). Early sipping of alcohol has been associated with increased odds of consuming full drinks, getting drunk, and drinking heavily later in adolescence (Jackson 2015). Consumption of at least a standard drink of alcohol at or before age 13 has been associated with an increased risk of frequent binge drinking in late secondary school (Aiken 2018). Even a single occasion of alcohol intoxication can have serious short- and long-term consequences (Courtney 2009; Quinn 2011). Internationally, guidelines for low-risk alcohol consumption include recommendations for young people (in Australia under the age of 18 years, and in the US under the age of 21) not to drink at all (NHMRC 2009; USDHHS 2015).

Although the use of alcohol is common among young people, some groups can be identified as being at elevated risk of heavy use due to a range of social, peer, and family factors. Livingston and colleagues report that young people who have had their first drink by age 13 are almost twice as likely to engage in very high-risk drinking when aged 16 to 24 (Livingston 2008). Parents who allow their children to consume alcohol in adult-supervised settings in early adolescence are more likely to have children who experience harmful alcohol consequences in mid-adolescence (McMorris 2011). Further, parents who themselves have heavy drinking occasions are more likely to have children who report heavy drinking occasions (Hingson 2014), and parental substance use and family history of alcoholism have been identified as predictors of adolescent substance use in longitudinal studies (Alati 2014; Chassin 1996; Cranford 2010; White 2000; Wills 2003). Evidence is mixed in relation to the association between socioeconomic disadvantage and risk of adolescent alcohol consumption (Hanson 2007). Some reports show drinking and drunkenness associated with lower levels of disadvantage or higher levels of household income (Reboussin 2010; Richter 2009). Other reports show higher levels of baseline problem drinking among low socioeconomic status communities (Caria 2011; Lowry 1996).

\section{Description of the intervention}

Despite the influence of peers and society during adolescence (Carter 2007; Patton 2004), parenting and home environment factors are important influencers of development (Steinberg 2001), as well as predictors of alcohol consumption and other substance use (Carter 2007; Simons-Morton 2009; Turrisi 2010; Wang 2009). Maternal and paternal knowledge of their child's friends and whereabouts is reported to act as a protective factor against substance use and to mediate the variability in substance use by grade and ethnic background (Wang 2009). This protective effect is suggested to act via an influence on peer group selection (Engels 2007; Wang 2009), the transmission of family attitudes and values (White 2010), and parental monitoring (knowledge of their child's whereabouts) (Jimenez-Iglesias 2013).

In 1994, the US Institutes of Medicine adopted a framework for the classification of mental health and substance use prevention interventions as universal, selective, or indicated/targeted (Mrazek 1994; Springer 2006). Universal prevention strategies address the entire population within a particular setting. Selective interventions are delivered to subgroups of individuals based on their membership in a group that has an elevated risk of developing problems. Indicated interventions address vulnerable individuals and help them in dealing and coping with their individual personality traits that make them more vulnerable to escalating drug use (EMCDDA 2015).

Although intervention programmes are usually classified as belonging to one of these three broad groups, the classification can be regarded as a continuum, with obvious overlap between groups. In the 2010 report "Fair Society, Healthy Lives", commissioned by the United Kingdom (UK) government to identify the most evidence-based strategies for reducing health inequalities, a key recommendation was to extend the focus of preventive activities beyond the most disadvantaged, to encompass the full spectrum of the social gradient. It was stated that to "reduce the steepness of the social gradient in health, actions must be universal, but with a scale and intensity that is proportionate to the level of disadvantage" (Marmot 2010).

Applied to alcohol prevention efforts, this 'proportionate universalism' can be interpreted as the need to conduct universal prevention programmes, but to also include more targeted (selective and indicated) interventions for higher-risk groups. Parenting skills are recognised as a key factor in the prevention of adolescent alcohol consumption and other substance use. The proportionate universalism approach maintains that all parents should be given opportunities for support and help to develop appropriate protective parenting skills, and that some parents who demonstrate a particular risk profile or who have particular 
needs (e.g. have vulnerable children) should be offered increasingly targeted (and increasingly costly) interventions (Heginbotham 2012; Marmot 2010). For this reason, this review is not limited to universal interventions, but will incorporate those classified as selective and indicated.

Classification of interventions in the present review is based on their target population, whether all parents (universal) or a select group based on characteristics of parents or their children (selective and indicated). In the context of familybased interventions for alcohol use in young people, universal interventions target parents of all children given the inherent risk of alcohol use among all sectors of the population. These interventions will likely aim to delay the initiation of alcohol use, or to reduce the frequency or volume of use among children of participating parents. Selective interventions are those targeting parents whose children have an elevated risk of substance use due to social or family risk factors. Such risk factors include low socioeconomic status or family income, along with parental alcohol consumption, alcoholism, or other substance use. Similarly, these interventions will likely aim to delay initiation or reduce consumption. Indicated interventions are defined as those that target parents or families whose children are already identified as drinkers. These interventions will more likely aim to reduce levels of consumption or the frequency of binge drinking and/or to reduce alcohol-related harms.

Parent- and family-based programmes for the prevention of alcohol use are often appended to school curricula-based interventions for young people, but may also be designed as stand-alone programmes. Such programmes frequently focus on parent-child communication and relationship building. Common elements across many programmes include focus on social competence skills, parental involvement with children, and self-regulation, although the target population and the intensity and mode of delivery are highly varied.

\section{How the intervention might work}

The theoretical basis for family-based interventions is that young people whose parents adopt appropriate parenting strategies are likely to develop positive social norms and to resist the negative external influences of peers and society. In this context, positive parenting strategies include rule-setting, appropriate communication, monitoring, and conveying positive values and attitudes (Ryan 2010). Family- and parent-based interventions for adolescent substance use operate indirectly, with the mechanism of effect working via parents rather than through a programme delivered directly to young people as the target population. As such, the developmental trajectory of particular behaviours, for example, alcohol use, is changed via improved family or parent socialisation practices (Foxcroft 2014).

\section{Why it is important to do this review}

Previous Cochrane Reviews have separately covered universal family-based programmes (Foxcroft 2011a), as well as school-based and multi-component interventions (Foxcroft 2011b; Foxcroft 2011c, respectively), for alcohol misuse among young people that incorporate family-based interventions. The most recent of these reviews was completed with studies published up to July 2010. Since the time of that review, several trials have been published, reporting on other family-based preventive programmes, and in many cases using innovative approaches including online delivery.

As well as updating the previous review (Foxcroft 2011a), the current review extends beyond universal interventions to include those classified as selective and indicated, in keeping with the concept of proportionate universalism.

Although parents and families are influential and provide a key target for intervention, family-based programmes are often expensive to run and challenging from a recruitment and engagement perspective (Haggerty 2006). It is important to gather evidence of their effectiveness, and of the differential effectiveness of various components of these programmes, to inform policy and funding decisions.

\section{O B JECTIVES}

To assess the effectiveness of universal, selective, and indicated family-based prevention programmes in preventing alcohol use or problem drinking in school-aged children (up to 18 years of age).

Specifically, on these outcomes, the review aimed:

- to assess the effectiveness of universal family-based prevention programmes for all children up to 18 years ('universal interventions');

- to assess the effectiveness of selective family-based prevention programmes for children up to 18 years at elevated risk of alcohol use or problem drinking ('selective interventions'); and

- to assess the effectiveness of indicated family-based prevention programmes for children up to 18 years who are currently consuming alcohol, or who have initiated use or regular use ('indicated interventions').

\section{METHODS}

\section{Criteria for considering studies for this review Types of studies}

Randomised controlled trials (RCTs) including cluster-randomised trials (C-RCTs).

\section{Types of participants}

Parents or guardians/carers of young people up to 18 years (of school age). For this review, we defined young people as children and adolescents and excluded those transitioning to college due to differences in context and parenting roles. We included parents of young people who have not previously consumed alcohol, currently consume alcohol, or have heavy or problematic alcohol use. Young people were also included as participants in some interventions and in the context of data collection.

\section{Types of interventions}

Any universal, selective, or indicated family-based psychosocial or educational prevention intervention.

We defined universal prevention strategies as those addressing the entire population without selection of children based on characteristics that may increase their risk of alcohol use or problem drinking, for example, those offered to all parents of children attending a school. 
We defined selective interventions as those delivered to a subgroup of children identified as having socio-demographic or other characteristics that put them at an elevated risk of alcohol use or problem drinking, for example, those delivered to families in which there is a history of substance use or mental health problems among parents, to those living in communities of low socioeconomic status, or to those engaging in delinquent behaviour.

We defined indicated interventions as those targeting a subgroup of children who currently use alcohol or who may have alcoholrelated problems.

We included prevention programmes that focused on alcohol as well as other drugs wherever alcohol outcomes were presented separately. We defined psychosocial interventions as interventions that specifically aim to develop psychological and social attributes and skills in parents or young people (e.g. parental monitoring, behavioural norms, peer resistance), so that young people are less likely to use alcohol. We defined educational interventions as those that specifically aim to raise awareness amongst parents and/or carers of how to positively influence young people, or of the risks of alcohol consumption, so that young people are less likely to use alcohol.

The comparison consisted of any alternative prevention programme (e.g. school-based, office-based, multi-component, other) where the parental component could be experimentally isolated (e.g. parent plus school compared to school only) or no programme.

\section{Types of outcome measures}

\section{Primary outcomes}

Any direct self-reported (by adolescents) measures of alcohol consumption or problem drinking. As an example, we considered the following outcomes to be relevant.

- Alcohol use (yes/no).

- Alcohol use (quantity, frequency).

- 'Binge' drinking (e.g. defined as drinking five or more drinks on any one occasion) (yes/no).

- Incidence of drunkenness.

Outcome measures related to psychological perception/attitudes or awareness of alcohol risks were deemed to be indirect; therefore we did not consider them in this review.

\section{Secondary outcomes}

Secondary outcomes could be measured through self-report (by adolescents or parents) or through police, juvenile justice, or medical records.

- Age of alcohol initiation.

- Age of drunkenness initiation.

- Alcohol-related problems or harms (e.g. drunk driving or any physical or social problem self-reported by adolescents as an alcohol-related consequence may be measured using a scale such as Rutgers Alcohol Problems Index or questions 7 to 10 of the Alcohol Use Disorders Identification Test (AUDIT)).
- Parent-reported or child-reported alcohol-related parenting behaviours (e.g. supply of alcohol, alcohol-specific communication, alcohol-specific rule-setting).

\section{Search methods for identification of studies}

\section{Electronic searches}

We searched the following databases, without restrictions by language or publication status, in June 2018. The search strategy is based on that used by Foxcroft 2011a but with the removal of terms that were designed to limit the previous review to universal interventions. Thus these searches were conducted afresh from the earliest available records with no limits placed on publication date.

- Cochrane Drugs and Alcohol Group's Specialised Register of Trials.

- Cochrane Central Register of Controlled Trials (CENTRAL, 2018 issue), in the Cochrane Library.

- MEDLINE (Ovid) (1966 to 30 June 2018).

- Embase (Embase.com) (1974 to 30 June 2018).

- Education Resource Information Center (ERIC; EBSCOhost) (1966 to 30 June 2018)

- PsycINFO (Ovid) (1806 to 30 June 2018).

- Google Scholar (modified MEDLINE search to account for 260 character limit).

- Project CORK (http://www.projectcork.org).

- ClinicalTrials.gov (clinicaltrials.gov/).

- International Clinical Trials Registry Platform (ICTRP) (apps.who.int/trialsearch/).

The subject strategies for databases were modelled on the search strategy designed for CENTRAL. Where appropriate, these were combined with subject strategy adaptations of the Cochrane highly sensitive search strategy for identifying RCTs and controlled clinical trials (as described in the Cochrane Handbook for Systematic Reviews of Interventions, Version 5.1.0, Box 6.4.b; Higgins 2011). Search strategies for major databases are provided in Appendix 1.

\section{Searching other resources}

We handsearched the reference lists of topic-related systematic reviews and included studies to identify potentially relevant citations (Dusendury 2000; Gates 2006; Hale 2014; Kuntsche 2016; Lemstra 2010; MacArthur 2012; Petrie 2007 Smit 2008; VermeulenSmit 2015; ). Unpublished reports, abstracts, dissertations, and brief and preliminary reports were eligible for inclusion.

\section{Data collection and analysis}

\section{Selection of studies}

Pairs of independent reviewers (including CG, AW, LW, JT, TS, ES) completed broad screening of titles and abstracts of all identified records (screening level 1). Afterwards, the same pairs independently assessed full-text reports of all potentially relevant records that passed the initial screen. We resolved differences in opinion arising at both screening levels through discussion and involvement of a third review author for resolution where required. Reasons for exclusion of full-text articles were recorded and are reported in Characteristics of excluded studies. 


\section{Data extraction and management}

Pairs of review authors independently extracted relevant data using an a priori defined data extraction form (CG, AW, ES, TS), and one review author (AW) entered data into Review Manager 5 software (RevMan 2014). We resolved differences in opinion arising during data extraction through discussion and involvement of a third review author for resolution where required. We extracted the following information: numbers and characteristics of participants, setting, types of experimental and control interventions, length of follow-up, types of outcomes, outcome data (sample sizes, means, standard deviations, odds ratios, confidence intervals as available), country of origin, and methodological characteristics associated with the assessment of risk of bias (randomisation procedures, blinding, data collection procedures, attrition, outcome reporting, and analysis characteristics associated with clustered studies).

\section{Assessment of risk of bias in included studies}

For each study included in the review, two review authors independently assessed the risk of bias (CG, AW, TS, ES, MK). We performed the risk of bias assessment for RCTs in this review using the criteria recommended in the Cochrane Handbook for Systematic Reviews of Interventions (Higgins 2011). The recommended approach addresses seven specific domains, namely, sequence generation and allocation concealment (selection bias), blinding of participants and providers (performance bias), blinding of outcome assessor (detection bias), incomplete outcome data (attrition bias; less than $20 \%$ loss of participants with no differential attrition between experiment groups was regarded as low risk), selective outcome reporting (reporting bias), and other sources of bias (contamination bias). For C-RCTs, we also assessed risk of recruitment bias, baseline imbalances, loss of clusters, incorrect analysis, and compatibility with individually randomised trials (herd effect). The first part of the tool involves describing what was reported to have happened in the study. The second part of the tool involves assigning a judgement related to the risk of bias for that entry, in terms of low, high, or unclear risk. To make these judgements, we used the criteria indicated by the Cochrane Handbook for Systematic Reviews of Interventions, as adapted to the addiction field. See Appendix 2 for details.

\section{Grading of evidence}

We assessed the overall quality of the evidence for the primary outcome of each study using the GRADE system. The Grading of Recommendation, Assessment, Development and Evaluation Working Group (GRADE) developed a system for grading the quality of evidence (GRADE 2004; Guyatt 2008; Guyatt 2011), which takes into account issues related not only to internal validity (risk of bias) but also to external validity, such as directness, consistency, imprecision of results, and publication bias. The 'Summary of findings' tables present the main findings of a review in a transparent and simple tabular format. In particular, they provide key information concerning the quality of evidence, the magnitude of effect of the interventions examined, and the sum of available data on the main outcomes.

In this review, we present the 'Summary of findings' tables based on type of intervention programme (universal, selective, indicated) and type of comparison (intervention vs intervention as well as comparative effectiveness trials). Summary tables cover those comparisons where sufficient studies were available to enable meta-analytical pooling.
The GRADE system uses the following criteria in assigning grades of evidence.

- High: we are very confident that the true effect lies close to that of the estimate of the effect.

- Moderate: we are moderately confident in the effect estimate: the true effect is likely to be close to the estimate of the effect, but there is a possibility that it is substantially different.

- Low: our confidence in the effect estimate is limited: the true effect may be substantially different from the estimate of the effect.

- Very low: we have very little confidence in the effect estimate: the true effect is likely to be substantially different from the estimate of effect.

Comparisons of RCTs begin with a 'high' rating and are downgraded based on serious (-1) or very serious (-2) limitation to study quality; important inconsistency $(-1)$; some $(-1)$ or major $(-2)$ uncertainty about directness; imprecise or sparse data $(-1)$; and high probability of reporting bias $(-1)$.

\section{Measures of treatment effect}

We calculated treatment effects using RevMan 2014 where possible.

\section{Dichotomous outcome data}

We analysed dichotomous outcomes by calculating the risk ratio (RR) for each trial, with the uncertainty in each result expressed using 95\% confidence intervals (Cls).

\section{Continuous outcome data}

We analysed continuous outcomes by calculating mean differences (MDs) if all studies used the same measurement scale, or standardised mean differences (SMDs) if studies used different measurement scales, each with $95 \% \mathrm{Cls}$. If data in small studies were skewed, we assessed the implications for outcomes on a caseby-case basis.

\section{Unit of analysis issues}

We ascertained additional validity threats regarding appropriate unit of analysis depending on whether randomisation was implemented at an individual or cluster level. We assessed clusterrandomised trials in the review for unit of analysis error. For studies that did not adjust for clustering, we calculated design effects and effective sample sizes using available study data and reported intraclass correlations (ICCS). Where ICCs were not available, we used a mean ICC calculated from reported ICCS of included studies to calculate effective sample sizes before inclusion in meta-analysis (Higgins 2011). We included studies with more than two trial arms in the meta-analysis by selecting the most appropriate intervention and comparison (e.g. family-based intervention vs no-intervention control, with no data taken from a classroom-based intervention arm). We included studies in two separate meta-analyses if they included a family-based intervention arm that could be compared separately with a no-intervention or standard care arm and a family and adolescent intervention.

\section{Dealing with missing data}

Where important summary data or study level characteristics were missing, we attempted to contact the authors of those included studies. Where standard deviations were missing from 
continuous data, we scanned studies for any other statistics (Cls, standard errors, $T$ values, $P$ values, $F$ values) that allowed for their calculation. Where available, we reported outcomes of trials reporting an intention-to-treat analysis.

\section{Assessment of heterogeneity}

Assessment of heterogeneity involved inspecting each included study for variability in study populations (baseline characteristics), interventions (target/focus, mode of delivery), and outcome measures (tools, instruments, scales, and outcome definitions). We considered methodological heterogeneity by inspecting variability in study design and risk of bias. Where acceptable homogeneity was found within subgroups (based on age of children, type of intervention, or substance targeted), we conducted meta-analysis for subgroups of studies. We assessed statistical heterogeneity using the $\mathrm{Chi}^{2}$ test and its $\mathrm{P}$ value, by visually inspecting the forest plots, and by using the $I^{2}$ statistic. A P value of the test lower than 0.10 or an $I^{2}$ statistic of at least $50 \%$ indicated significant statistical heterogeneity.

\section{Assessment of reporting biases}

We used funnel plots (plots of the effect estimate from each study against the sample size or effect standard error) to indicate possible publication bias. We used tests for funnel plot asymmetry only when a minimum of 10 studies were included in the meta-analysis, as fewer than 10 studies would render the power of the tests too low to distinguish chance from real asymmetry.

\section{Data synthesis}

We calculated pooled standardised mean differences (to account for heterogeneity of outcome measures) for each comparison using a random-effects model with a generic inverse variance weighting method (RevMan 2014). We calculated standardised mean differences for all outcome measures to maximise comparability, and we used the generic inverse variance method, which allows for inclusion of studies reporting data in a range of forms including both continuous and dichotomous outcomes along with those reporting odds ratios, risk ratios, or differences between means. We selected post-intervention values over changes from baseline data for inclusion in the meta-analysis, to reduce the risk of selective reporting and to maximise the number of studies that could be pooled.

We synthesised studies that provided suitable data for pooling in meta-analysis grouped by outcome. Due to small numbers of studies in each comparison, we explored effects by type of prevention intervention (i.e. universal, selective, or indicated) in subgroup analyses. Depending on study numbers in each comparison, selective and indicated interventions may be grouped together to represent more targeted approaches in contrast to universal ones; these would be regarded as further along the scale of proportionate universalism (Marmot 2010). We grouped outcomes as measuring alcohol use prevalence (measures of the prevalence of any alcohol consumption or a specified threshold of consumption such as the prevalence of drinking at least once per month); frequency (measures of the number of occasions of use in a given period); or volume (measures of the number of drinks in a given period). When studies reported multiple alcohol outcomes in one of these categories, we selected the most conservative measure capturing small or infrequent levels of use (e.g. the frequency of any drinking was selected in preference to the frequency of drunkenness, if both were available). Studies could contribute to multiple meta-analyses if they reported eligible outcomes in more than one category. From studies that reported multiple follow-up points, we extracted data from the longest follow-up period up to four years for inclusion in meta-analyses.

We selected study estimates that adjusted for potential confounding variables for inclusion in meta-analysis over estimates that did not adjust for potential confounding variables, when available. Similarly, we selected C-RCT study estimates that were adjusted for clustering for inclusion in meta-analyses over unadjusted estimates. For those C-RCTs that did not adjust for clustering, we adjusted study estimates using a mean ICC from other included studies and the effective sample size used in metaanalysis. We pooled separately studies that compared two or more alternative interventions, enabling experimental isolation of the parent intervention component.

In all instances where data could not be pooled in a metaanalysis, we have provided a narrative summary of the trial findings according to the review objectives.

\section{Subgroup analysis and investigation of heterogeneity}

We investigated the extent of heterogeneity through visual examination of forest plots and through use of the $\mathrm{Chi}^{2}$ statistic, the $\mathrm{P}$ value, and the $\mathrm{I}^{2}$ statistic. Where there was evidence of heterogeneity $\left(I^{2}\right.$ statistic $\left.>50 \%\right)$, we investigated the potential source of heterogeneity through subgroup analyses. Specifically, we conducted subgroup analyses based on the type of prevention intervention (universal, selective, indicated), the intensity of the intervention (considering duration and level of face-to-face involvement), the characteristics of participants (ethnicity and gender), and the length of follow-up (less than 12 months, or between 12 months and 4 years).

\section{Sensitivity analysis}

We performed sensitivity analysis of the main review outcomes, removing trials judged to be at high risk of bias (graded as high on three or more 'Risk of bias' measures). For C-RCTs, two or more ratings of high risk on any of the five cluster-specific risk of bias domains contributed one high risk rating to the overall assessment.

\section{RES U LTS}

\section{Description of studies}

See Characteristics of included studies, Characteristics of excluded studies, and Characteristics of ongoing studies.

\section{Results of the search}

See the CONSORT flow diagram (Figure 1). 
Figure 1. Study flow diagram.

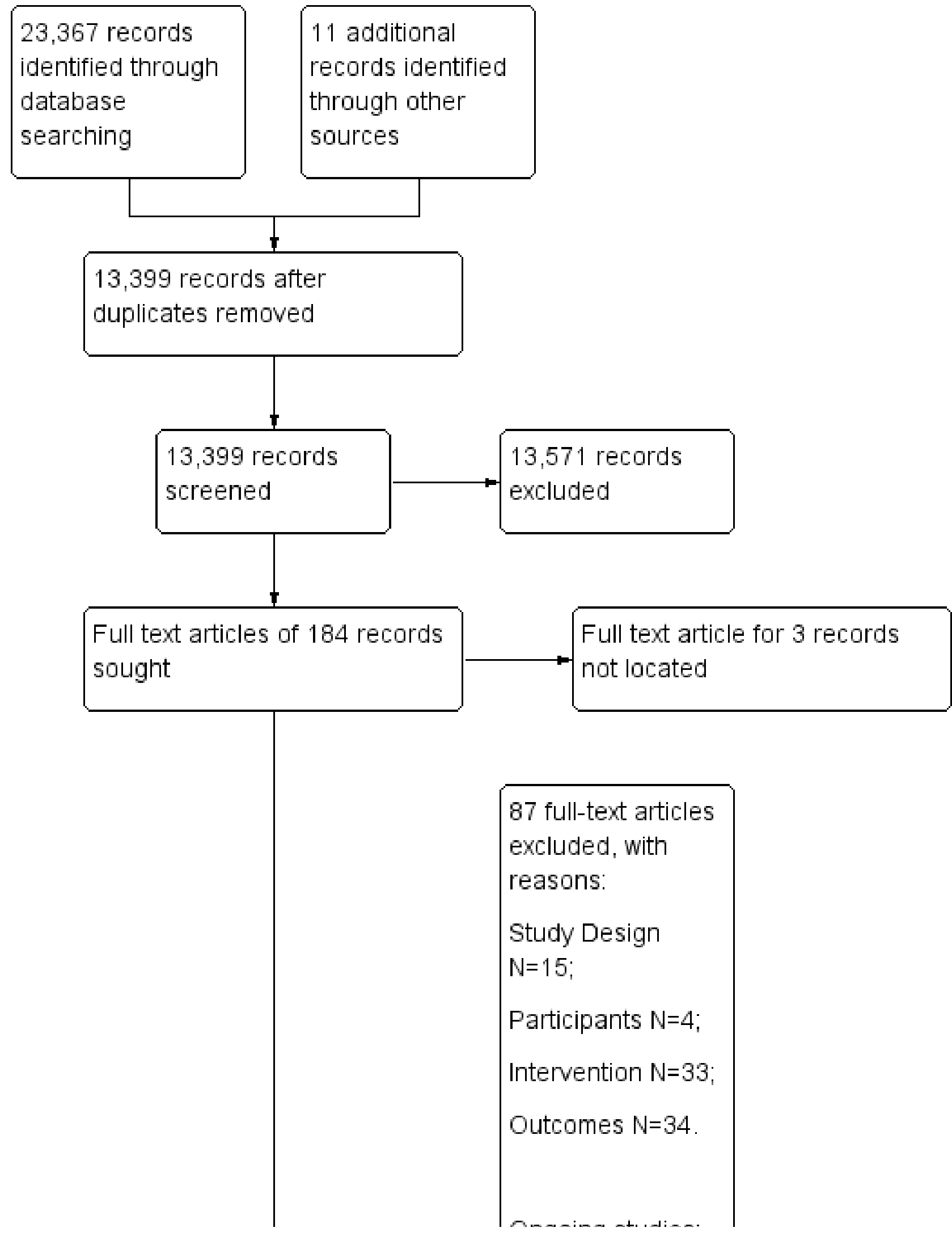


Figure 1. (Continued)

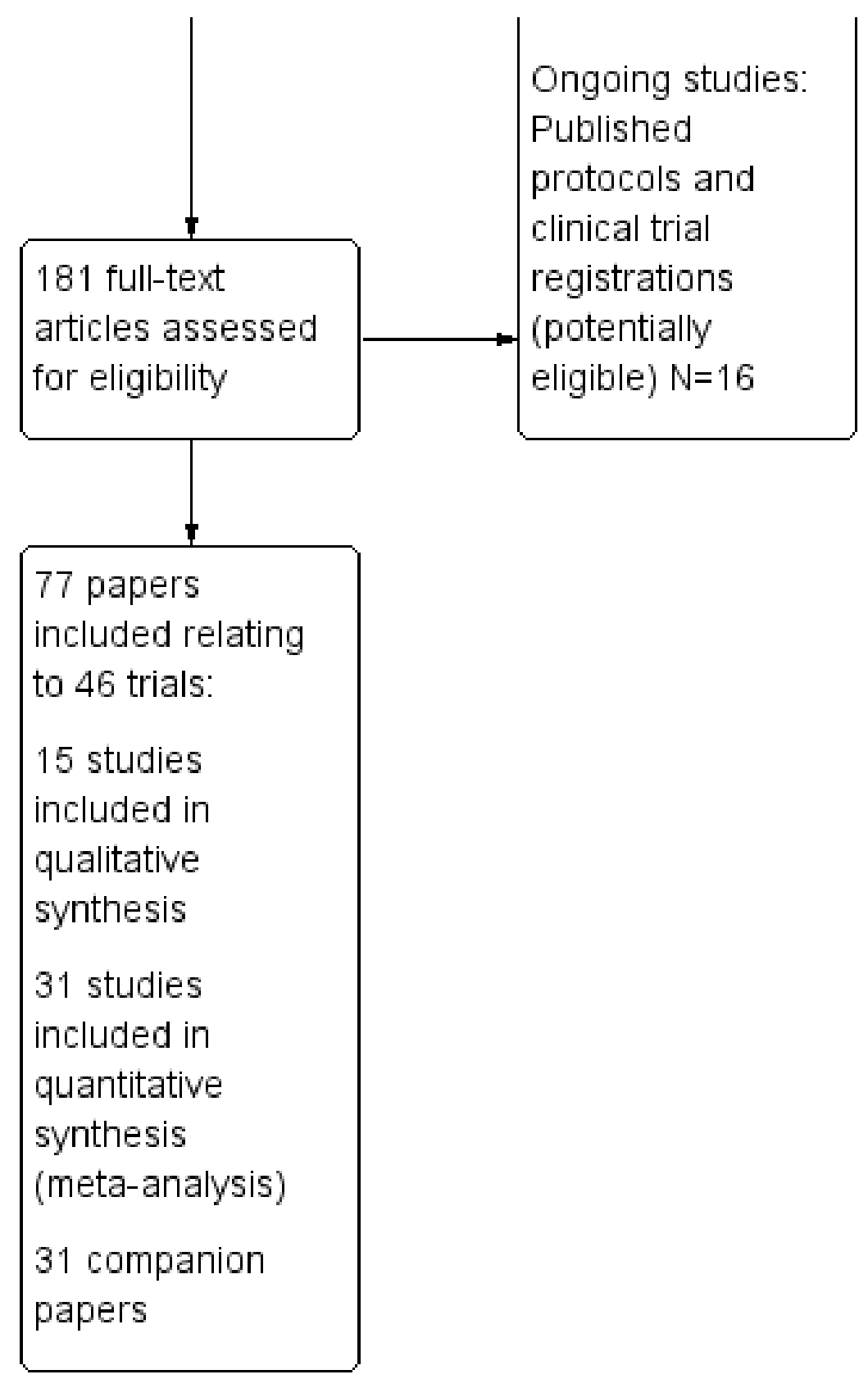

The search strategy resulted in a total of 23,367 citations, and we identified a further 11 studies by checking the reference lists of relevant systematic reviews. After removal of duplicate records, 13,399 records remained. Screening of titles and abstracts revealed 184 studies for full-text review and formal inclusion or exclusion. Of these, 46 papers met the inclusion criteria as primary studies (Arnaud 2016; Baldus 2016; Bauman 2002; Bodin 2011; Brody 2006; Catalano 1999; Cordova 2012; Dembo 2001; Estrada 2017; Fang 2010; Fosco 2013; Foxcroft 2017; Furr-Holden 2004; Haggerty 2007; Koning 2009; Liddle 2008; Linakis 2013; Loveland-Cherry 1999; Mares 2016; Mason 2012; Milburn 2012; O'Donnell 2010; Perry 2003; Prado 2012; Reddy 2002; Riesch 2012; Schinke 2004; Schinke 2009a; Schinke 2009b; Schinke 2009c; Schinke 2011; Skarstrand 2014; Spirito 2011; Spirito 2015; Spirito 2017; Spoth 1999a; Spoth 2002; Stanger 2017; Stevens 2002; Stormshak 2011; Valdez 2013; Werch
2008; Winters 2012; Wolchik 2002; Wu 2003; Wurdak 2017), and a further 31 as companion papers to included trials.

\section{Included studies}

A description of the included studies appears in the Characteristics of included studies tables. We included 46 studies with 39,822 participants (or families) randomised across the 46 included trials. Thirty-one studies were RCTs, 25 of which compared an intervention group versus a no intervention control group or a 'usual care' group (Baldus 2016; Bauman 2002; Catalano 1999; Cordova 2012; Dembo 2001; Estrada 2017; Fang 2010; Fosco 2013; Haggerty 2007; Linakis 2013; Loveland-Cherry 1999; Mason 2012; Milburn 2012; O'Donnell 2010; Prado 2012; Schinke 2009a; Schinke 2009b; Schinke 2009c; Schinke 2011; Spirito 2017; Stanger 
2017; Stormshak 2011; Valdez 2013; Wolchik 2002; Wurdak 2017), and six of which compared the effectiveness of two different family- or parent-focused interventions (Liddle 2008; Schinke 2004; Spirito 2011; Spirito 2015; Werch 2008; Winters 2012). The other 15 included studies were C-RCTs, 10 of which compared an intervention group versus a no intervention control group (Arnaud 2016; Bodin 2011; Brody 2006; Foxcroft 2017; Furr-Holden 2004; Koning 2009; Mares 2016; Riesch 2012; Skarstrand 2014; Spoth 1999a), and five of which were comparative effectiveness trials (Perry 2003; Reddy 2002; Spoth 2002; Stevens 2002; Wu 2003). In total, we classified 12 studies as comparative effectiveness trials, usually with more than two trial arms, including a comparison of a family/parent intervention coupled with adolescent intervention components versus the adolescent components alone. In such studies, experimental isolation of the parent component for analysis purposes was possible (Koning 2009; Liddle 2008; Perry 2003; Reddy 2002; Schinke 2004; Spirito 2011; Spirito 2015; Spoth 2002; Stevens 2002; Werch 2008; Winters 2012; Wu 2003).

Twenty-seven of the included studies tested the impact of interventions classified as universal, targeting all children or families; 12 were selective, targeting groups at elevated risk; and seven were classified as indicated, targeting families where young people were already using alcohol. Of studies comparing universal interventions, 13 were C-RCTs, with nine using schools as the unit of randomisation, one using county (Brody 2006), one using communities (Foxcroft 2017), one using classrooms (FurrHolden 2004), and one using paediatric clinics (Stevens 2002). Among selective and indicated interventions, only one study in each category was a C-RCT, with the selective study randomising community centres (Wu 2003), and the indicated study randomising paediatric emergency departments (Arnaud 2016). The 14 universal RCTs randomised participants at the level of adolescent-parent dyads ( $\mathrm{n}=7$; Bauman 2002; Estrada 2017; Linakis 2013; Schinke 2009a; Schinke 2009b; Schinke 2009c; Schinke 2011), families ( $n=4$; Fosco 2013; Haggerty 2007; Loveland-Cherry 1999; O'Donnell 2010), adolescents ( $n=1$; Werch 2008), communities ( $n=1$; Schinke 2004), or parents ( $n=1$; Wurdak 2017). The selective RCTs randomised individual families ( $\mathrm{n}=7$; Catalano 1999; Mason 2012; Milburn 2012; Prado 2012; Spirito 2015; Stormshak 2011; Wolchik 2002), adolescents ( $n=2$; Cordova 2012; Dembo 2001), or dyads ( $n=2$; Baldus 2016; Fang 2010). The six indicated RCTs randomised at the level of the family ( $n=3$; Spirito 2011; Spirito 2017; Valdez 2013), or at the level of the adolescent ( $n=3$; Liddle 2008; Stanger 2017; Winters 2012).

\section{Country}

Twenty-nine trials were undertaken in the United States; 16 studies examined universal interventions (Estrada 2017; Haggerty 2007; Linakis 2013; Loveland-Cherry 1999; O'Donnell 2010; Schinke 2004; Schinke 2009a; Schinke 2009b; Schinke 2009c; Schinke 2011; Werch 2008; Perry 2003; Riesch 2012; Spoth 1999a; Spoth 2002; Stevens 2002), 11 studies selective interventions (Catalano 1999; Cordova 2012; Dembo 2001; Fang 2010; Mason 2012; Milburn 2012; Prado 2012; Spirito 2015; Stormshak 2011; Wolchik 2002; Wu 2003), and six studies targeted interventions (Liddle 2008; Spirito 2011; Spirito 2017; Stanger 2017; Valdez 2013; Winters 2012). Two trials were conducted in the Netherlands (both universal C-RCTs; Koning 2009; Mares 2016), two in Sweden (both universal C-RCTs; Bodin 2011; Skarstrand 2014), one in Poland (a universal C-RCT; Foxcroft 2017), three in Germany (one universal RCT - Wurdak 2017; one selective
RCT - Baldus 2016; and one indicated C-RCT - Arnaud 2016), and one in India (a universal C-RCT - Reddy 2002).

\section{Participants}

Ethnicity of participants was mixed. Twelve trials included exclusively or over-represented specific ethnic groups. Four studies exclusively - Wu 2003, Brody 2006 - or predominantly - FurrHolden 2004, Liddle 2008 - involved African American participants. Three further studies included a close to 50:50 ratio of African American and Caucasian (or other) participants (Dembo 2001; Haggerty 2007; Riesch 2012). Four studies involved only Hispanic or Mexican American participants (Cordova 2012; Estrada 2017; Prado 2012; Valdez 2013), and one study involved only Asian American participants (Fang 2010). A further 12 studies involved participants from a mix of ethnic backgrounds: two mostly Caucasian and African American (Loveland-Cherry 1999; Werch 2008); four mostly Causasian and Hispanic/Latino (Mason 2012; Milburn 2012; Spirito 2011; Spirito 2015); and six a mixture of all these groups (O'Donnell 2010; Schinke 2004; Schinke 2009b; Schinke 2009c; Schinke 2011; Stormshak 2011). Twelve studies included a mix of ethnicities but predominantly Caucasian American (Bauman 2002; Catalano 1999; Linakis 2013; Loveland-Cherry 1999; Perry 2003; Schinke 2009a; Spirito 2017; Spoth 1999a; Spoth 2002; Stanger 2017; Winters 2012; Wolchik 2002). One study involved a broader range of ethnic groups including a minority of Native American and Pacific Islander participants (Fosco 2013). The remaining nine studies did not target particular ethnic groups nor report particular cohort breakdowns.

The age of children targeted through the interventions ranged from 5 to 17 years (average approximately 13 years). Furr-Holden 2004 involved very young children, with an average age of 6.2 years, and Stanger 2017 involved the oldest cohort, with an average age of 16.1 years. In general, the average age of adolescent participants was higher in trials of selective (approximately 13 years) and indicated (approximately 15.5 years) trials than in trials of universal interventions (approximately 12 years). Six studies exclusively targeted girls, four of which provided universal interventions (O'Donnell 2010; Schinke 2009a; Schinke 2009b; Schinke 2009c), and two of which gave selective interventions (Fang 2010; Schinke 2011).

\section{Recruitment and eligibility}

Of the universal interventions, a majority recruited participants via schools ( $n=17$; Bodin 2011; Brody 2006; Estrada 2017; Fosco 2013; Furr-Holden 2004; Haggerty 2007; Koning 2009; LovelandCherry 1999; Mares 2016; O'Donnell 2010; Perry 2003; Reddy 2002; Riesch 2012; Skarstrand 2014; Spoth 1999a; Spoth 2002; Werch 2008). Five studies used community advertisements such as newspapers, flyers, and "craigslist" (Schinke 2009a; Schinke 2009b; Schinke 2009c; Schinke 2011; Wurdak 2017); two recruited through community agencies such as after school care and social organisations (Foxcroft 2017; Schinke 2004), one through paediatric emergency departments (Linakis 2013), one through paediatric clinics (Stevens 2002), and one through telephone recruitment (Bauman 2002).

Among the selective interventions, three studies recruited participants specifically from low socioeconomic status or atrisk areas, with two of these recruiting through schools (Baldus 2016; Stormshak 2011), and one through community organisations and recreation centres (Wu 2003). Four recruited youth who had identified behaviour problems (recruited through schools - 
Cordova 2012), delinquency (recruited through the juvenile justice system - Dembo 2001; Prado 2012), or emotional or behavioural disorder (referred from mental health clinics, truancy courts, or response to advertisements - Spirito 2015). Three studies targeted children of at risk parents, with one recruiting families through a methadone clinic (Catalano 1999), one recruiting the children of depressed parents through health clinics (Mason 2012), and one recruiting the children of divorced parents identified through court records (Wolchik 2002). One study targeted families with a homeless adolescent recruited through community organisations such as shelters (Milburn 2012). One further study targeted girls from minority ethnic groups identified through community advertisements (Fang 2010).

The seven indicated interventions all involved youth who were already identified as using or abusing alcohol. Two studies recruited participants who attended a paediatric emergency department or trauma centre after an alcohol-related incident (Arnaud 2016; Spirito 2011), one recruited gang-affiliated youths via a street-based outreach approach (Valdez 2013), and one recruited youth who had been identified in a school setting as abusing alcohol and other drugs (Winters 2012). The remaining three studies relied on referrals from truancy courts, schools, juvenile justice, or welfare agencies (Liddle 2008; Spirito 2017; Stanger 2017).

\section{Setting and mode of delivery}

Researchers delivered interventions in a range of settings including the child's school, the child's family home, and the Internet or delivered print material. Of the universal interventions, they delivered eight to parents via print materials or audio CD sent by post or via email, or sent home with children (Bauman 2002; Mares 2016; O'Donnell 2010; Perry 2003; Reddy 2002; Schinke 2004; Werch 2008; Wurdak 2017), with one sent by post ( $n=1$; O'Donnell 2010); four were computer mediated (Schinke 2009a; Schinke 2009b; Schinke 2009c; Schinke 2011); two involved presentations or workshops at the child's school (Bodin 2011; Fosco 2013); and ten involved face-to-face sessions, with a combination of group/individual/family sessions delivered at the school or in a community venue (Brody 2006; Estrada 2017; Foxcroft 2017; FurrHolden 2004; Haggerty 2007; Koning 2009; Riesch 2012; Skarstrand 2014; Spoth 1999a; Spoth 2002), or at individual parent/family sessions provided in the family home (Loveland-Cherry 1999), or in a healthcare setting (Linakis 2013; Stevens 2002).

Of the selective interventions, one was delivered via CD-ROM and Internet (Fang 2010), and ten via face-to-face sessions using a combination of group, individual parent, and family approaches (Baldus 2016; Catalano 1999; Cordova 2012; Dembo 2001; Mason 2012; Milburn 2012; Prado 2012; Spirito 2015; Stormshak 2011; Wolchik 2002). Individual parent and family sessions were most commonly delivered in the family home. One study involved faceto-face sessions for youth only and a 20-minute video for parents (Wu 2003). All indicated interventions were delivered through faceto-face sessions with parents and youth separately or together, or by a combination of both.

Across all trials, programme intensity varied from six sessions of 20 minutes' duration delivered over three years (Bodin 2011), to twice weekly 90-minute meetings, a five-hour retreat, and group workshops occurring over a nine-month period (Catalano 1999). In general, the selective and indicated interventions were of a consistently higher intensity than the universal ones, with all but one - Fang 2010 - involving at least one face-to-face session. Faceto-face interventions varied in duration/frequency from a single session in Arnaud 2016 to weekly sessions over periods ranging from five (in Milburn 2012) to 16 weeks (in Valdez 2013) to annual sessions provided over three years (Bodin 2011; Fosco 2013). Interventions delivered by other means also varied, with some spread over four weeks (Wurdak 2017), and others up to six months (Bauman 2002). Duration of follow-up ranged from immediate posttest to 15 years post intervention (Wolchik 2002). A small number of trials reported follow-up beyond four years post randomisation ( $n=2$; Furr-Holden 2004; Wolchik 2002); we did not include these trials in the meta-analysis.

\section{Interventions and comparisons}

Although the interventions implemented varied in intensity, duration, and approach, all targeted alcohol or other drug use, and generally did so by promoting positive parenting approaches or by enhancing parent-child relationships. The interventions focused on elements such as communication, family dynamics, rule-setting, and risk management. Of the 46 included studies, 23 included a separate youth component in the form of a classroom curriculum or other adolescent-focused resource $(n=4$; Catalano 1999; Perry 2003; Reddy 2002; Schinke 2004), or individual or group youth sessions (and/or involvement in family sessions) as part of face-toface interventions ( $n=18$; Catalano 1999; Cordova 2012; Dembo 2001; Estrada 2017; Foxcroft 2017; Loveland-Cherry 1999; Milburn 2012; Prado 2012; Riesch 2012; Skarstrand 2014; Spirito 2011; Spirito 2015; Spoth 2002; Stanger 2017; Stevens 2002; Valdez 2013; Winters 2012; Wolchik 2002).

\section{Universal interventions}

Of the universal interventions, eight targeted alcohol specifically (Bodin 2011; Brody 2006; Koning 2009; Loveland-Cherry 1999; Mares 2016; Schinke 2004; Werch 2008; Wurdak 2017), 12 targeted substance use more generally (Bauman 2002; Foxcroft 2017; FurrHolden 2004; Linakis 2013; Riesch 2012; Schinke 2009a; Schinke 2009b; Schinke 2009c; Schinke 2011; Spoth 1999a; Spoth 2002; Stevens 2002), five targeted problem behaviours and substance use (Fosco 2013; Haggerty 2007; Perry 2003; Skarstrand 2014; Estrada 2017), and the remainder targeted alcohol as well as tobacco (Bauman 2002), sexual behaviour (O'Donnell 2010), or tobacco alone (Reddy 2002).

Six universal studies used the original structure or an adaptation of the Strengthening Families Program (SFP), which is based on the Social Development Model and aims to enhance parent and child interactions to reduce risk factors for substance use and substance use initiation (Brody 2006; Foxcroft 2017; Riesch 2012; Skarstrand 2014; Spoth 1999a; Spoth 2002). Investigators in each of these studies ran multiple face-to-face sessions over a period of several weeks. Generally in the first hour, parents and adolescents attended separate workshops before coming together for family workshops in the second hour. Workshop sessions were focused on skill-building and relationship development, using role-plays and games. The most common model for the Strengthening Families Program consisted of seven sessions over seven weeks and was used in four studies (Brody 2006; Foxcroft 2017; Riesch 2012; Spoth 2002). One study adapted the SFP to include two parts; part 1 included six separate parent and youth sessions and one family session, and part 2 involved four separate sessions and one joint 
session (Skarstrand 2014). One selective study also used the SFP in its seven-session format with four booster sessions (Baldus 2016).

One of these studies assessed the SFP as a complement to a 15session classroom-based curriculum of Life Skills Training (LST) for children in grades seven and eight (Spoth 2002), thereby investigating effects of the SFP via a comparative effectiveness approach (one arm was LST only, and the other LST plus SFP). Another study adopted a five-session model of the SFP, with children only attending one of these sessions, and compared this to the Preparing for Drug Free Years (PDFY) programme involving six sessions with separate parent and child training, as well as a final session with the family (Spoth 1999a). The remainder of these SFP-based studies compared the programme versus no programme (Riesch 2012; Skarstrand 2014), or versus an attention control involving the distribution of information leaflets via mail (Brody 2006; Foxcroft 2017).

Seven other universal studies also involved face-to-face sessions with small groups of individual parents or families (Estrada 2017; Fosco 2013; Furr-Holden 2004; Haggerty 2007; Linakis 2013; Loveland-Cherry 1999; Stevens 2002). Three studies involved group seminars or workshops for parents, with one providing nine sessions (Furr-Holden 2004), one eight sessions (Estrada 2017), and one seven sessions (Haggerty 2007). Another study involved individual motivational interviewing-based sessions with parents who were attending an emergency department with a child for a non-alcohol- or drug-related issue (Linakis 2013). This programme also included telephone booster sessions and mailings and was compared with an enhanced usual care approach including mailing of brochures about the influence of parents on adolescents. One universal study used home visits with families to deliver a motivational interviewing/social cognitive theorybased intervention and to overcome barriers to assessment of parent elements of interventions and/or parent attendance at school events, with three home sessions plus boosters delivered to families from three school districts and compared with a no program control group randomised at the family level (LovelandCherry 1999). The final universal study involving face-to-face sessions delivered at home did so only for families who were identified through the school-based part of the programme as being at risk (Fosco 2013). As such, this component of the intervention was regarded as the selective component. The universal component of the intervention involved a family resource centre in schools and delivery of special interest face-to-face seminars for parents. This intervention was compared with a no programme control

Two universal studies used the Orebro Prevention Program or adaptation (Bodin 2011; Koning 2009). This programme involves presentations to parents at schools and the development of a set of agreed rules among parents. Both studies compared the programme versus a no intervention control, and Koning et al included three arms, also comparing the effectiveness of a student intervention $(\mathrm{SI})$ ) with and without the parent intervention $(\mathrm{PI} ; \mathrm{SI}$ +/-PI versus PI) (Koning 2009).

The remaining 11 universal studies used either paper or computerbased modules with no face-to-face component. Eight studies involved mailing material to parents (e.g. booklets, postcards, audio-CDs; Bauman 2002; Mares 2016; O'Donnell 2010; Perry 2003; Reddy 2002; Schinke 2004; Werch 2008; Wurdak 2017). Four of these studies compared parent mailings versus a no program or waitlist control (Bauman 2002; Mares 2016; O'Donnell 2010; Wurdak 2017), and four were comparative effectiveness trials (Perry 2003; Reddy 2002; Schinke 2004; Werch 2008), in which parent mailings were assessed as a complement to, or in comparison to, an alternate intervention such as a classroom curriculum (Perry 2003; Reddy 2002), a CD-ROM programme for adolescents (Schinke 2004), or a set of alternate adolescent postcards (Werch 2008). Four studies were based on mother-daughter education and a cognitive-behavioural skills training approach using computeror CD-ROM-mediated sessions, all compared with a no program control (Schinke 2009a; Schinke 2009b; Schinke 2009c; Schinke 2011).

\section{Selective interventions}

Of the selective interventions, only one study targeted alcohol specifically (Stormshak 2011), with three targeting alcohol and substance use (Fang 2010; Mason 2012; Spirito 2015), and eight targeting alcohol/substance use (Baldus 2016; Catalano 1999; Cordova 2012; Dembo 2001; Milburn 2012; Prado 2012; Wolchik 2002; Wu 2003), along with other problem behaviours such as unsafe sex in Prado 2012 or selling drugs in Wu 2003.

Less variation existed in the interventions delivered in selective studies compared to universal interventions. Ten studies used faceto-face sessions with a mixture of group, parent only, or family counselling based on the principles of motivational interviewing, cognitive-behavioural therapy, or similar counselling approaches (Baldus 2016; Catalano 1999; Cordova 2012; Dembo 2001; Mason 2012; Milburn 2012; Prado 2012; Spirito 2015; Stormshak 2011; Wolchik 2002). The 'intensity' of these interventions ranged from a single family session with assessment task and boosters delivered by mail, as in Spirito 2015, to multiple home visits with families. Two studies used the Family Check-Up intervention, comprising assessment, feedback, and motivational interviewing principles (Spirito 2015; Stormshak 2011). One study involved five sessions with youth and parents at home (Milburn 2012), two studies involved ten such visits (Dembo 2001; Mason 2012), and one study involved nine group sessions as well as ten family sessions (Family Unidas; Cordova 2012). One study involved 11 group sessions with mothers as well as two individual sessions tailored to the intervention (Wolchik 2002). One study involved a total of 54 contact hours per family, with a mixture of group and individual sessions and a parent retreat (Catalano 1999). Only two selective studies did not involve face-to-face contact with parents, with one using a nine-session web-based programme targeting mothers' relationships with their daughters (Fang 2010), and the other complementing a face-to-face youth programme with a 20-minute video for parents (Wu 2003).

Most of these selective studies compared an intervention versus either standard practice (e.g. standard methadone clinic, standard referral processes; Catalano 1999; Milburn 2012; Prado 2012), an enhanced 'usual care' condition (Baldus 2016; Cordova 2012; Dembo 2001; Wolchik 2002), or no programme (Fang 2010; Mason 2012; Stormshak 2011). Two selective studies were comparative effectiveness trials that compared the intervention versus an alternative, such as a psychoeducational session in Spirito 2015 or a child-focused intervention in Wu 2003.

\section{Indicated interventions}

Of the indicated interventions, one specifically targeted alcohol (Spirito 2011); three targeted risk behaviours and drug use 
(including alcohol) (Arnaud 2016; Liddle 2008; Valdez 2013); one targeted substance use including alcohol (Winters 2012); and two targeted alcohol and marijuana use (Spirito 2017; Stanger 2017). In all cases, we considered for this review only outcomes related specifically to alcohol.

All indicated interventions included face-to-face sessions based on motivational interviewing (Arnaud 2016; Liddle 2008; Spirito 2011; Spirito 2017), cognitive-behavioural therapy (Stanger 2017), or brief intervention principles (Valdez 2013; Winters 2012). Intensity varied, with two studies involving a single family motivational interviewing session, as well as a youth component (Spirito 2011; Spirito 2017); one involving two sessions with youth and one with a parent (Winters 2012); and one involving 16 family therapy sessions (Valdez 2013). These interventions were compared with usual care (e.g. referrals, social and behavioural services; in Arnaud 2016 and Valdez 2013) or a no programme control (Winters 2012), and four studies compared the effectiveness of family or parent therapy with adolescent motivational interviewing (Spirito 2011), cognitivebehavioural therapy (Liddle 2008), or psychoeducation (Spirito 2017). One indicated study compared abstinence-based incentives in the intervention group versus attendance-based incentives in the control group (Stanger 2017).

\section{Outcomes}

We grouped outcome measures used in meta-analysis as prevalence, frequency, or volume. Twenty studies reported measures of prevalence (Baldus 2016; Bauman 2002; Bodin 2011; Brody 2006; Catalano 1999; Cordova 2012; Foxcroft 2017; FurrHolden 2004; Haggerty 2007; Koning 2009; Mares 2016; O'Donnell 2010; Prado 2012; Reddy 2002; Riesch 2012; Skarstrand 2014; Spoth 1999a; Spoth 2002; Stevens 2002; Wu 2003). These studies included those assessing 'initiation' of or any alcohol in the child's lifetime (Baldus 2016; Bauman 2002; Brody 2006; Foxcroft 2017; FurrHolden 2004; Haggerty 2007; Mares 2016; Reddy 2002; Riesch 2012; Skarstrand 2014; Spoth 1999a; Spoth 2002; Stevens 2002), some of which also reported this measure for a cohort of baseline nondrinkers (Baldus 2016; Bauman 2002; Brody 2006); those reporting the lifetime prevalence of drunkenness (Skarstrand 2014); and those reporting the prevalence of weekly use (Bodin 2011), or use in the last 90 days (Cordova 2012; Prado 2012), 6 months (Catalano 1999; Wu 2003), or 12 months (O'Donnell 2010).

Seventeen studies reported alcohol use frequency outcomes (Arnaud 2016; Dembo 2001; Estrada 2017; Fang 2010; Liddle 2008; Linakis 2013; Milburn 2012; Perry 2003; Schinke 2004; Schinke 2009b; Spirito 2011; Stanger 2017; Valdez 2013; Werch 2008; Winters 2012; Wolchik 2002; Wurdak 2017). These studies all reported on the number of occasions of drinking, with the exception of one study that reported on occasions of binge drinking (Arnaud 2016). Most studies reported frequency of use in the past 30 days (Dembo 2001; Fang 2010; Liddle 2008; Linakis 2013; Schinke 2004; Schinke 2009b; Spirito 2011; Valdez 2013; Werch 2008; Wolchik 2002; Wurdak 2017), and others reported use over time periods of 90 days (Estrada 2017; Milburn 2012; Winters 2012), 36 weeks (Stanger 2017), or 12 months (Perry 2003).

Ten studies reported alcohol use volume outcomes (Arnaud 2016; Fosco 2013; Loveland-Cherry 1999; Mason 2012; Schinke 2009a; Schinke 2009c; Schinke 2011; Spirito 2015; Spirito 2017; Stormshak 2011). Most reported on the number of drinks consumed in the past 30 days (Arnaud 2016; Fosco 2013; Mason 2012; Schinke 2009a;
Schinke 2009c; Schinke 2011; Spirito 2017; Stormshak 2011), and two studies used a quantity-frequency scale calculated over 3 months in Spirito 2015 and over 12 months in Loveland-Cherry 1999.

\section{Excluded studies}

A total of 179 records remained after title and abstract screening, of which 176 full-text articles were located for further review. We considered 85 articles to be ineligible after assessment of the full text (reasons for exclusion were study design $(\mathrm{N}=15)$, participants $(N=4)$, interventions $(N=34)$, and outcomes $(N=32))$. See Characteristics of excluded studies for further details.

\section{Studies awaiting classification}

We could not determine the eligibility of three trials, as no full text was available. See Characteristics of studies awaiting classification.

\section{Ongoing studies}

We identified 16 ongoing trials by their published protocol or by a clinical trial registration, for which neither published nor unpublished data were available (Characteristics of ongoing studies). These include five trials regarded as universal, two as selective, and nine as indicated.

The universal trials included a C-RCT comparing a range of health interventions for adolescents, including one related to alcohol and delivered to parents (Ford 2015); an RCT of the Strengthening African American Families STEPS program targeting 11-15 yearolds (Kogan 2018), an RCT comparing the Family Matters and Strengthening Families programmes (vs a no program control group) among families with an 11- or 12-year-old child attending Kaiser Permanente medical centres (Miller 2009); an RCT of a webbased 'Smart Choices 4 teens' program targeting alcohol and sexual behaviour (Miller 2018), and a C-RCT of a UK adaptation of the Strenthening Families Program, comparing a seven-session model versus usual care (Segrott 2014).

The selective trials included an RCT testing the effects of a parenting programme for Latino families versus a waitlist control (Allen 2012), along with an RCT testing an American Indian adaptation of the Strengthening Families programme with orientation towards cultural traditions of Anishinaabe communities versus a no intervention control group (Whitbeck 2016).

The indicated intervention trials included an RCT trialling home-based behavioural therapy for adolescents with disruptive behaviour disorder and regular substance use versus usual care (Bukstein 2006); a C-RCT testing an extensive prevention programme involving adolescent and parent components and an indicated component for youth with symptoms of mental health or substance use problems versus treatment as usual (Conrod 2017); an RCT testing the feasibility of a motivational enhancement therapy intervention for adolescents with and without a parenting wisely programme for parents and a drug education programme for adolescents with and without a parenting wisely programme for parents among adolescents with drug-related charges (Hops 2012); an RCT of enhanced contingency management for adolescents with a current substance use disorder, with and without a parent management training programme (McCart 2017); an RCT of adolescent brief intervention and an e-parenting skills intervention for parents of adolescents admitted to a trauma service with 
a positive screening for alcohol or drug use compared to brief intervention alone (Mello 2016); an RCT of multi-dimensional family therapy compared to family motivational interviewing and a standard care control group for adolescents presenting to the emergency room or trauma unit with alcohol problems (Rowe 2010); an RCT of a contingency management programme compared to usual care for youth in the justice system with a newly opened probation case (Sheidow 2017); an RCT of a computer-assisted motivational interviewing programme and an online parenting wisely programme for adolescents in the justice system who have a positive result for marijuana use on intake (Spirito 2017b); and an
RCT comparing adolescent group therapy versus transitional family therapy for adolescents with a DSM-IV diagnosis of alcohol abuse or dependence (Stanton 2007).

\section{Risk of bias in included studies}

The assessment results of risk of bias for the included studies are presented in Figure 2 and Figure 3 . None of the 46 included studies were at low risk in all risk-of-bias domains (Higgins 2011). Overall eight studies were regarded as high risk (with three or more 'high' ratings) for the purpose of sensitivity analysis.

\section{Figure 2. Risk of bias graph: review authors' judgements about each risk of bias item presented as percentages} across all included studies.

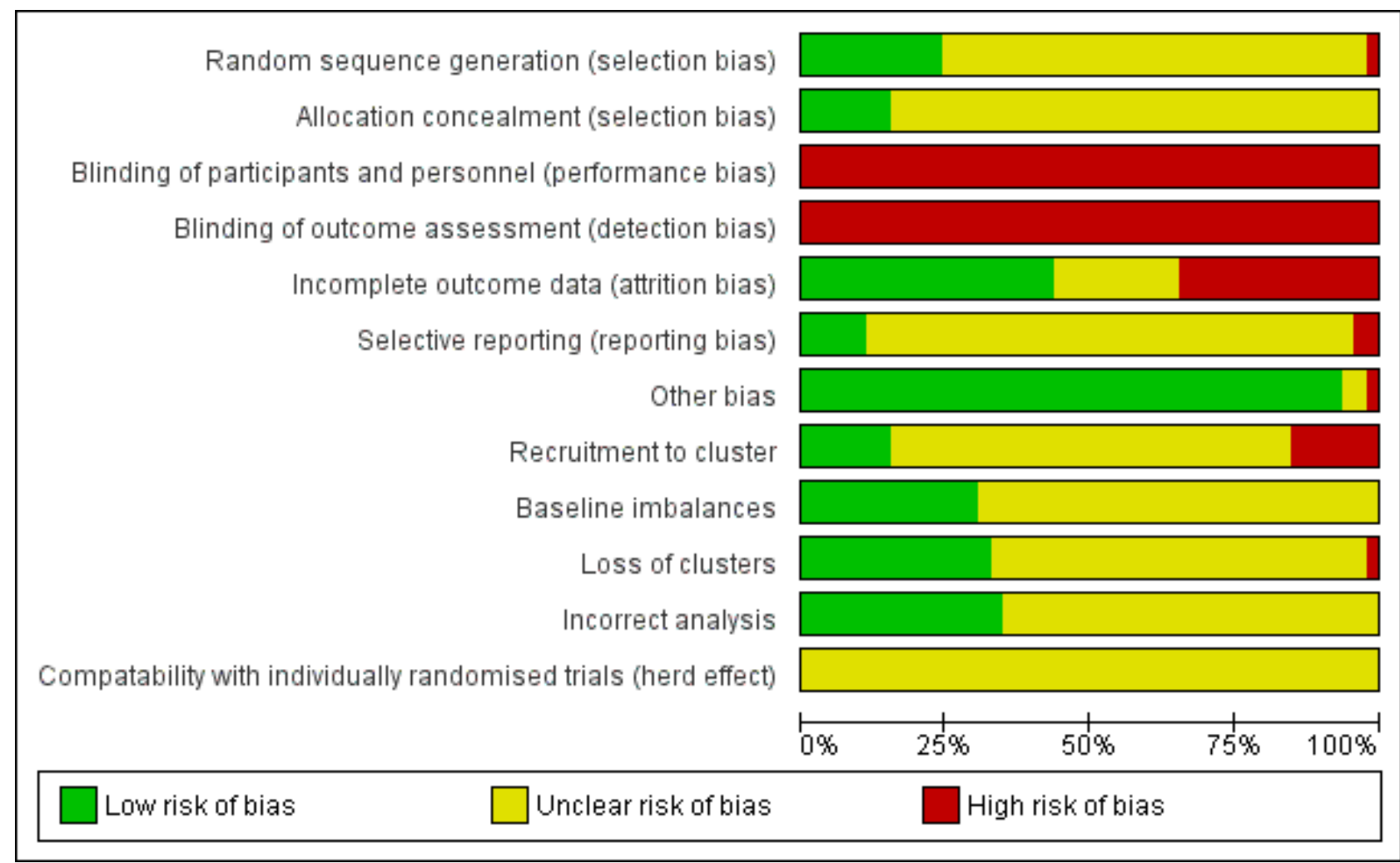


Figure 3. Risk of bias summary: review authors' judgements about each risk of bias item for each included study.

\begin{tabular}{|c|c|c|c|c|c|c|c|c|c|c|c|c|}
\hline & 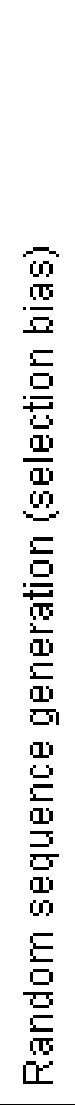 & 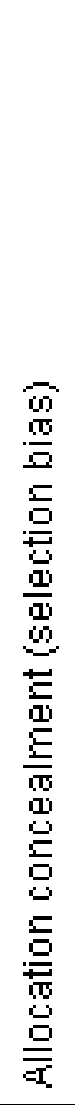 & 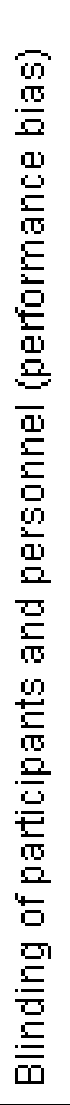 & 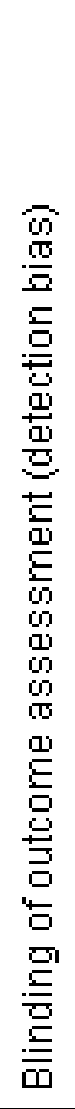 & 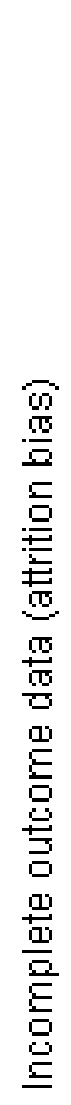 & 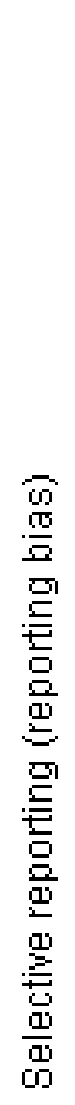 & $\begin{array}{l}\stackrel{0}{\mathbf{\omega}} \\
\stackrel{5}{\mathbf{5}} \\
\stackrel{5}{5}\end{array}$ & 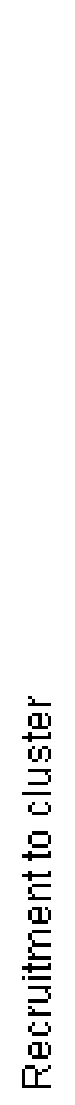 & 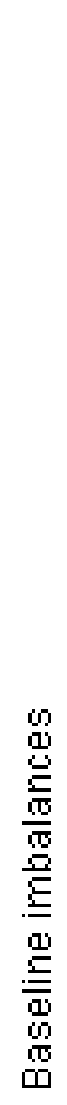 & 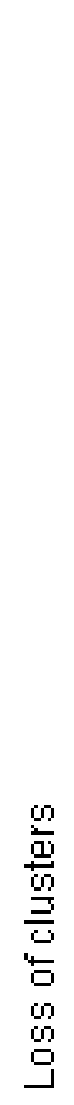 & 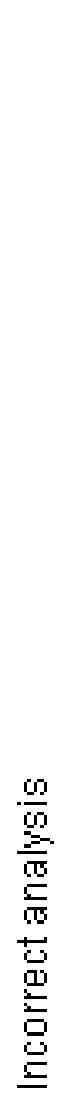 & 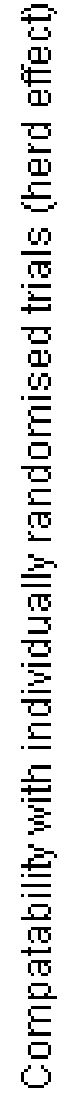 \\
\hline Arnaud 2016 & $?$ & $?$ & & & . & $?$ & $?$ & 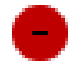 & $?$ & + & + & $?$ \\
\hline Baldus 2016 & $?$ & $?$ & & & + & $?$ & + & $?$ & $?$ & $?$ & $?$ & $?$ \\
\hline Bauman 2002 & $?$ & $?$ & & & $?$ & $?$ & + & $?$ & $?$ & $?$ & $?$ & $?$ \\
\hline Bodin 2011 & + & + & & & & + & & + & + & + & + & $?$ \\
\hline Brody 2006 & $?$ & $?$ & & & $?$ & $?$ & & & + & + & + & $?$ \\
\hline Catalano 1999 & $?$ & $?$ & & & $A$ & $?$ & & $?$ & $?$ & $?$ & $?$ & $?$ \\
\hline Cordova 2012 & + & $?$ & & & 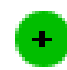 & $?$ & + & $?$ & $?$ & $?$ & $?$ & $?$ \\
\hline Dembo 2001 & $?$ & $?$ & & & + & 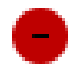 & & $?$ & $?$ & $?$ & $?$ & $?$ \\
\hline Estrada 2017 & $?$ & $?$ & & & & $?$ & + & $?$ & $?$ & $?$ & $?$ & $?$ \\
\hline Fang 2010 & $?$ & $?$ & & & . & $?$ & 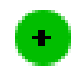 & $?$ & $?$ & $?$ & $?$ & $?$ \\
\hline
\end{tabular}


Figure 3. (Continued)

\begin{tabular}{|c|c|c|c|c|c|c|c|c|c|c|c|c|}
\hline \multirow{2}{*}{$\begin{array}{l}\text { ' cory }<410 \\
\text { Fosco } 2013\end{array}$} & . & $\cdot$ & $\gamma$ & 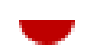 & $\sigma$ & $\cdot$ & $\sigma$ & $\cdot$ & $\cdot$ & $\cdot$ & . & $\cdot$ \\
\hline & $?$ & $?$ & & & $?$ & $?$ & $?$ & $?$ & $?$ & $?$ & $?$ & $?$ \\
\hline Foxcroft 2017 & $\odot$ & + & & & $\odot$ & - & $\odot$ & $\odot$ & + & $\odot$ & $\oplus$ & $?$ \\
\hline Furr-Holden 2004 & $?$ & $?$ & & & $\odot$ & + & 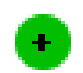 & + & + & 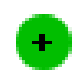 & + & $?$ \\
\hline Haggerty 2007 & $?$ & $?$ & & & + & ? & + & $?$ & $?$ & $?$ & $?$ & $?$ \\
\hline Koning 2009 & $?$ & + & & & $\odot$ & ? & + & $\odot$ & + & $\Theta$ & + & ? \\
\hline Liddle 2008 & $?$ & $?$ & & & - & ? & + & $?$ & $?$ & $?$ & $?$ & $?$ \\
\hline Linakis 2013 & $?$ & $?$ & 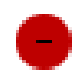 & & $\odot$ & $?$ & 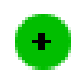 & $?$ & $?$ & $?$ & $?$ & $?$ \\
\hline Loveland-Cherry 1999 & $?$ & $?$ & & & $\odot$ & ? & 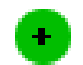 & $?$ & $?$ & $?$ & $?$ & $?$ \\
\hline Mares 2016 & + & $?$ & & & - & + & + & + & + & + & + & $?$ \\
\hline Mason 2012 & $?$ & $?$ & & & + & ? & + & $?$ & $?$ & $?$ & $?$ & $?$ \\
\hline Milburn 2012 & + & + & & & - & $?$ & + & $?$ & $?$ & $?$ & ? & $?$ \\
\hline O'Donnell 2010 & $?$ & $?$ & & & $\odot$ & ? & + & $?$ & $?$ & $?$ & $?$ & $?$ \\
\hline Perry 2003 & $?$ & $?$ & & & + & $?$ & + & $?$ & + & + & + & $?$ \\
\hline Prado 2012 & $?$ & + & & & $\odot$ & $?$ & 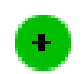 & $?$ & $?$ & $?$ & $?$ & $?$ \\
\hline Reddy 2002 & $?$ & $?$ & & & + & ? & + & $?$ & + & + & + & $?$ \\
\hline Riesch 2012 & $?$ & $?$ & & & $\odot$ & ? & $\odot$ & - & + & $\odot$ & $\oplus$ & $?$ \\
\hline Schinke 2004 & $?$ & $?$ & & & $?$ & $?$ & $\oplus$ & + & + & $\oplus$ & $\oplus$ & $?$ \\
\hline Schinke 2009a & $?$ & $?$ & & & + & ? & + & $?$ & $?$ & $?$ & $?$ & $?$ \\
\hline Schinke 2009b & $?$ & $?$ & & & + & $?$ & + & $?$ & $?$ & $?$ & $?$ & $?$ \\
\hline Schinke $2009 \mathrm{c}$ & $?$ & $?$ & & & $?$ & $?$ & $\odot$ & $?$ & $?$ & $?$ & $?$ & $?$ \\
\hline \multirow[t]{2}{*}{ Schinke 2011} & $?$ & $?$ & & & $?$ & ? & $\odot$ & $?$ & $?$ & $?$ & $?$ & $?$ \\
\hline & & & & & & & & & & & & \\
\hline
\end{tabular}


Figure 3. (Continued)

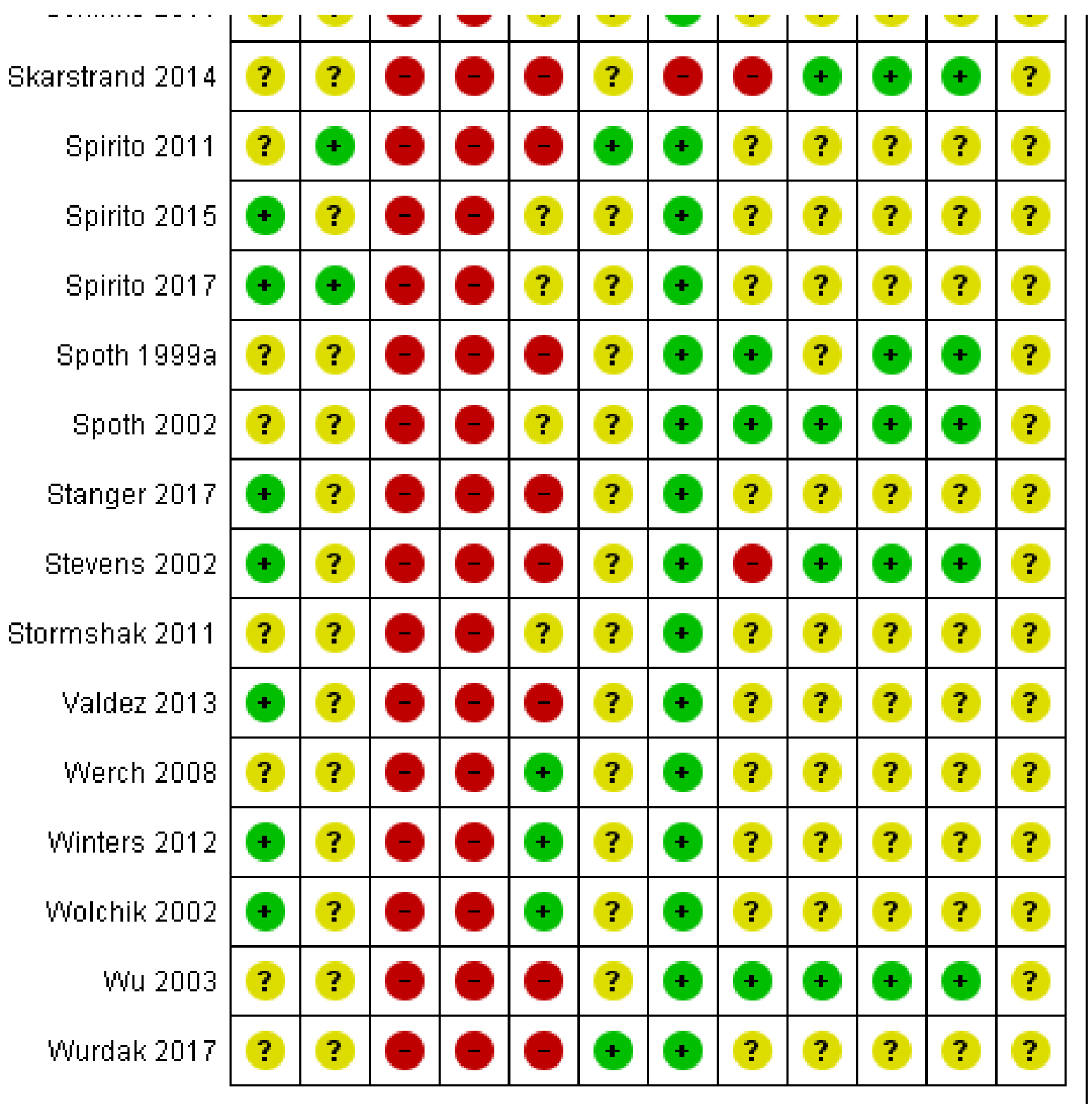

\section{Allocation}

\section{Random sequence generation}

We rated 11 of the 46 studies at low risk of bias for random sequence generation. Four studies used a computer-generated random number sequence (Mares 2016; Stevens 2002; Valdez 2013; Wolchik 2002), four studies used urn randomisation (Cordova 2012; Spirito 2015; Spirito 2017; Winters 2012), one study used minimum likelihood allocation (Stanger 2017), and two studies used a coin toss (Bodin 2011; Milburn 2012). We judged the method of sequence generation in one study to be high risk, as four of 20 communities were not randomised and their data were retained (Foxcroft 2017). For the remaining 34 studies, the method of sequence generation was unclear.

\section{Allocation concealment}

Of the 46 studies, only seven provided sufficient detail to establish that participant allocation to experimental groups was concealed from those conducting the research; we rated these as having low risk of selection bias for this domain (Bodin 2011; Foxcroft 2017; Koning 2009; Milburn 2012; Prado 2012; Spirito 2011; Spirito 2017). We were unable to make a judgement on the remaining 39 studies using the details provided; therefore, those studies had unclear risk of selection bias with regard to allocation concealment.

\section{Blinding}

In all 46 studies, blinding of participants and programme deliverers (performance bias) and blinding of outcome assessment (detection bias) was not achievable due to the nature of the interventions tested and because the outcomes were self-reported; therefore, 
we rated these studies as having high risk of performance and detection bias.

\section{Incomplete outcome data}

We rated 20 studies at low risk of bias for incomplete outcome data, as they reported less than $20 \%$ loss of participants and showed no differential attrition between experimental groups (Arnaud 2016; Baldus 2016; Catalano 1999; Cordova 2012; Dembo 2001; Estrada 2017; Fang 2010; Furr-Holden 2004; Haggerty 2007; Linakis 2013; Mason 2012; O'Donnell 2010; Perry 2003; Prado 2012; Reddy 2002; Schinke 2009a; Schinke 2009b; Werch 2008; Winters 2012; Wolchik 2002). Sixteen studies had high risk of bias due to high attrition rates $(>20 \%)$ or had less than $20 \%$ loss of participants but unequal attrition between experiment groups (Bodin 2011; Foxcroft 2017; Koning 2009; Liddle 2008; Loveland-Cherry 1999; Mares 2016; Milburn 2012; Riesch 2012; Skarstrand 2014; Spirito 2011; Spoth 1999a; Stanger 2017; Stevens 2002; Valdez 2013; Wu 2003; Wurdak 2017). We rated the remaining 10 studies as having unclear risk for incomplete outcome data, as details were insufficient to permit a judgement.

\section{Selective reporting}

Five studies had low risk of reporting bias, as outcomes reported were consistent with the prespecified clinical trial registries and/ or the study protocol (Bodin 2011; Furr-Holden 2004; Mares 2016; Spirito 2011; Wurdak 2017). We judged two studies to be at high risk of reporting bias - the first as a direct comparison of the intervention group versus the control group was not presented (Dembo 2001), and the second because an outcome referred to in the protocol was not reported (Foxcroft 2017). We rated the remaining 39 studies as having unclear risk for incomplete outcome data, as details were insufficient to permit a judgement.

\section{Other potential sources of bias}

We assessed the potential for contamination bias as another potential source of bias in the 46 studies and judged only one study to be at high risk of contamination (Skarstrand 2014), as study authors noted that control schools were exposed to other alcohol interventions during the intervention period.

For the 16 C-RCTs, we assessed risk of recruitment bias, baseline imbalances, loss of clusters, incorrect analysis, and compatibility with individually randomised trials (herd effect). We considered seven studies to have low risk of recruitment bias based on appropriate recruitment techniques applied before allocation to clusters, seven to have high risk of bias (based on individual allocation to clusters occurring after randomisation), and the remaining studies to have unclear risk of bias (based on insufficient information). For baseline imbalances, we considered all studies to be at low risk of bias based on similar characteristics of groups at baseline (no baseline imbalances or imbalances accounted for in the analyses), except two studies that provided insufficient information to permit judgement (Arnaud 2016; Spoth 1999a). Only one study had high risk of bias for loss of clusters (Koning 2009). We judged all 16 studies as having low risk for incorrect analysis (based on adequate adjustment for the effect of clustering); however, review authors were required to adjust for clustering on behalf of the authors of four studies (i.e. we did not rate these studies as high risk because we were able to address the lack of adjustment for clustering; Brody 2006; Schinke 2004; Spoth 1999a; Wu 2003).
Information was insufficient to permit judgement of the herd effect for all studies.

\section{Effects of interventions}

See: Summary of findings for the main comparison Family/ parent interventions compared with control for reducing alcohol consumption in adolescents; Summary of findings 2 Family/ parent and adolescent interventions compared to adolescent only interventions for reducing alcohol consumption in adolescents

Examination of interventions tested, trial settings, and study populations suggested that the included studies were sufficiently homogenous, and we conducted meta-analyses by pooling data from trials where interventions and outcome measures were considered similar. Otherwise, we provided a narrative synthesis of trial findings.

For the purposes of meta-analysis, we pooled outcome measures as those measuring prevalence of alcohol use (including prevalence of lifetime use, any use in the last six months, and weekly use); frequency of use (including the number of occasions of use in the last 30 or last 90 days); and volume of use (including the number of drinks in the previous 30 days or a composite score generated from quantity and frequency measures).

\section{Primary outcomes}

Family-based intervention versus no intervention/standard care

\section{Prevalence of alcohol use}

\section{Any family-based intervention versus no intervention/standard care}

Overall, meta-analytical pooling of post-intervention data revealed that there was no intervention effect on the prevalence of alcohol use (standardised mean difference (SMD) 0.00, 95\% confidence interval $(\mathrm{Cl})-0.08$ to 0.08 ; studies $=12$; participants $=7490 ; \mathrm{I}^{2}=$ $57 \%$; Analysis 1.1; low-quality evidence). Results were similar in sensitivity analyses of studies with low risk of bias (SMD - $0.12,95 \%$ $\mathrm{Cl}-0.35$ to 0.11 ; studies $=4$; participants $=1733 ;\left.\right|^{2}=73 \%$; Analysis 1.4).

In subgroup analyses by prevention approach, results were similar.

\section{Universal family-based intervention versus no intervention/standard care}

Results show SMD $0.02(95 \% \mathrm{Cl}-0.06$ to 0.11 ; studies = 10 ; participants $=189 ; \mathrm{I}^{2}=60 \%$; Analysis 1.5$)$.

\section{Selective or indicated family-based intervention versus no intervention/standard care}

Results show SMD $-0.16(95 \% \mathrm{Cl}-0.36$ to 0.05 ; studies $=2$; participants $=357 ;\left.\right|^{2}=0 \%$; Analysis 1.6).

Effects on alcohol use prevalence were also consistent in subgroup analysis including the three studies whose participant population was regarded as a minority ethnic group (Brody 2006; Haggerty 2007; Riesch 2012) (SMD -0.20, 95\% Cl -0.42 to 0.02; studies = 3; participants $=325 ;\left.\right|^{2}=0 \%$; Analysis 1.9$)$.

Results remained consistent with the main analysis for studies regarded as providing low-intensity interventions (Analysis 1.7), those reporting on outcomes measured more than 12 months from randomisation or intervention delivery (Analysis 1.8), those primarily including an ethnic majority or Caucasian participants 
(Analysis 1.10), and those measuring lifetime or past six month use of any alcohol as opposed to heavy consumption (Analysis 1.11).

Several studies that could not be pooled measured alcohol use prevalence and reported mixed results in keeping with the metaanalysis. Furr-Holden 2004 collected data at five, six, and seven years post randomisation and reported that they found little evidence of the effect of their universal intervention on early onset of alcohol use. O'Donnell 2010 evaluated a universal parent education programme targeting parents of girls and reported that fewer girls in the intervention group drank at follow-up than in the control group (Adjusted Odds Ratio [AOR] 0.38, 95\% Cl 0.15 to $0.97 ; \mathrm{P}<0.05$ ) but did not report participant numbers by group to enable pooling. Two studies could not be pooled because the only data available conveyed the results of linear growth curve analyses. Cordova 2012 reported significant effects of their selective intervention on past 90 day alcohol use among US-born adolescents (regression coefficient/slope $[b]=-0.425, P$ $=0.017)$ but not among foreign-born adolescents $(\mathrm{b}=0.172, \mathrm{P}$ $=0.357$ ). Also, overall, the increase in alcohol use from baseline to 30-month follow-up was more modest than that observed in the control group. Prado 2012 report that although their selective Familias Unidas intervention was efficacious in reducing substance use, investigators found no intervention effect specific to current alcohol use $(b=-0.47, P=0.14)$

\section{Frequency of alcohol use}

\section{Any family-based intervention versus no intervention/standard care}

Overall, meta-analytical pooling of these studies revealed no intervention effects with substantial variability and heterogeneity $\left(\right.$ SMD $-0.31,95 \% \mathrm{Cl}-0.83$ to 0.21 ; studies $=8$; participants $=1835 ; \mathrm{I}^{2}$ =96\%; Analysis 1.2; very low-quality evidence). Sensitivity analysis including only studies with low risk of bias showed overall no intervention effects and heterogeneity that remained substantial (SMD 0.09, $95 \% \mathrm{Cl}-0.24$ to 0.43 ; studies $=5$; participants $=1488 ; \mathrm{I}^{2}$ $=87 \%$; Analysis 1.12).

\section{Subgroup analysis}

\section{Universal family-based intervention versus no intervention/standard} care

Universal studies measuring the effectiveness of parent/family interventions found no effects overall on frequency of alcohol use (SMD 0.18, 95\% Cl -0.40 to 0.75; studies = 3; participants $=1090 ; \mathrm{I}^{2}$ =92\%; Analysis 1.13).

\section{Selective or indicated family-based intervention versus no intervention/standard care}

We pooled selective and indicated trials together as only one selective study reported alcohol use frequency. In this metaanalysis, the interventions again had no effect, although the SMD indicated slight favouring of the intervention (SMD $-0.65,95 \% \mathrm{Cl}$ -1.64 to 0.33 ; studies $=5$; participants $=745 ;\left.\right|^{2}=97 \%$; Analysis 1.14 ) .

We observed a similar lack of effect in subgroup analyses including only studies with follow-up of outcome measures more than 12 months from intervention delivery or randomisation (SMD - 0.31 , $95 \% \mathrm{Cl}-0.83$ to 0.21 ; studies $=8$; participants $=1835 ; \mathrm{I}^{2}=96 \%$; Analysis 1.15) and those including ethnic majority or Caucasian participants (SMD 0.15, 95\% Cl -0.13 to 0.43 ; studies $=5$; participants $=799 ; I^{2}=71 \%$; Analysis 1.17). The effects of interventions delivered to ethnic minority groups approached significance (SMD -1.19, 95\% $\mathrm{Cl}-2.83$ to 0.46 ; studies $=3$; participants $=1037 ; \mathrm{I}^{2}=98 \%$; Analysis 1.16).

Studies that could not be pooled reported mixed effects on alcohol use frequency but overall were consistent with the lack of effects revealed in meta-analysis. Two studies reported data from growth curve analyses only (Dembo 2001; Estrada 2017). Dembo 2001 reported that youth who completed their selective Family Empowerment intervention reported getting drunk on alcohol less often than those who did not complete the programme. Data specific to intervention effects by group are not presented, with analysis focusing on predictors of drunkenness frequency. Estrada 2017 used growth curve analyses to examine trajectories of alcohol and drug use and reported no significant effects of their universal intervention on 90-day alcohol use. Wolchik 2002 reported outcomes beyond four years post randomisation, but we excluded these from meta-analysis due to heterogeneity. These study authors reported that they observed no significant alcohol effects of their selective mother plus child programme for children of divorce compared with control, but they noted significant effects of the mother only programme on alcohol consumption compared to control $(P=0.005)$.

One study provided no data regarding error of measurement, meaning that the data were not usable (Milburn 2012). Milburn 2012 report a significant effect of their selective cognitive-behavioural therapy intervention on the number of occasions of alcohol use in the three months before assessment (effect size $=0.38, \mathrm{P}=0.003$ ) but did not report whether alcohol was used by homeless youth in the trial. This study also reported that intervention participants reduced alcohol use significantly more than those given the control $(P=0.003)$.

\section{Volume of alcohol use}

\section{Any family-based intervention versus no intervention/standard care}

Overall, meta-analysis revealed a very small effect of parent/family interventions on alcohol consumption volume (SMD $-0.14,95 \% \mathrm{Cl}$ -0.27 to 0.00 ; studies $=5$; participants $=1825 ; I^{2}=42 \%$; Analysis 1.3 ; low-quality evidence). We performed sensitivity analysis that included studies rated as having low risk of bias and found that results remained consistent but showed increased variability and heterogeneity (SMD $-0.15,95 \% \mathrm{Cl}-0.32$ to 0.03 ; studies $=4$; participants $=1397 ;\left.\right|^{2}=52 \%$; Analysis 1.19).

Subgroup analyses by prevention approach yielded similar results.

Universal family-based intervention versus no intervention/standard care

We performed a subgroup analysis pooling universal studies measuring alcohol consumption volume, including three studies and maintaining a small positive effect of interventions (SMD -0.21, $95 \% \mathrm{Cl}-0.32$ to -0.10 ; studies $=3$; participants $=1481 ; \mathrm{I}^{2}=0 \%$; Analysis 1.20).

Selective or indicated family-based intervention versus no intervention/standard care

One selective study - Mason 2012 and one indicated study - Arnaud 2016 - were pooled together, revealing no overall effects of these interventions on alcohol volume (SMD $0.06,95 \% \mathrm{Cl}-0.15$ to 0.27 ; studies $=2$; participants $=344 ; 1^{2}=0 \%$; Analysis 1.21). 
We performed further subgroup analyses and found intervention effects remaining small for studies involving ethnic minority groups (SMD - $0.24,95 \% \mathrm{Cl}-0.36$ to -0.12 ; studies $=3$; participants $=1081$; $\mathrm{I}^{2}=0 \%$; Analysis 1.23) and for studies including females only (SMD $-0.25,95 \% \mathrm{Cl}-0.37$ to -0.13 ; studies $=2$; participants $=1053 ; \mathrm{I}^{2}$ $=0 \%$; Analysis 1.25). Effects were absent for studies measuring outcomes at or beyond 12 months from intervention delivery or randomisation (SMD $-0.16,95 \% \mathrm{Cl}-0.35$ to 0.02 ; studies $=3$; participants $=988 ; I^{2}=30 \%$; Analysis 1.22$)$ and for studies including ethnic majority or Caucasian participants (SMD $-0.01,95 \% \mathrm{Cl}-0.17$ to 0.15 ; studies $=2$; participants $=744 ; I^{2}=0 \%$; Analysis 1.24 ).

Three studies that could not be pooled reported intervention effects on alcohol use volume. Fosco 2013 reported data from latent growth curve analyses showing that their universal intervention was associated with an increase in students' self-regulation, which in turn was associated with a reduction in risk for antisocial behaviours including alcohol use that was significant at the $\mathrm{P}<0.05$ level. Stormshak 2011 conducted complier average causal effect (CACE) modelling to examine treatment effects on youth whose families engaged with the selective Family Check-Up intervention and reported that the intervention was successful in reducing the growth of alcohol use and other risk behaviours among middle school youth. Schinke 2009a did not report sample numbers by group for their universal programme, precluding pooling. Intervention by time interactions found that intervention arm girls reported less alcohol consumption than control arm girls over the past week $(P<0.01)$, month $(P<0.05)$, and year $(P<0.01)$.

\section{Family-based and adolescent intervention versus intervention with young people alone}

\section{Prevalence of alcohol use}

\section{Any family-based and adolescent interventions versus interventions} with young people alone

Overall, researchers found no effect of parent/family interventions on alcohol use prevalence compared to interventions with young people alone (SMD $-0.39,95 \% \mathrm{Cl}-0.91$ to 0.14 ; studies $=4$; participants $=5640 ; \mathrm{I}^{2}=99 \%$; Analysis 2.1 ; very low-quality evidence). The interventions trialled in these studies included both family/parent and youth components and were compared with youth components only.

Sensitivity analysis limited to those studies with low risk of bias yielded similar results (SMD $-0.61,95 \% \mathrm{Cl}-1.84$ to 0.63 ; studies $=2$; participants $=3891 ; I^{2}=100 \%$; Analysis 2.3 ).

\section{Subgroup analyses}

Universal family-based and adolescent interventions versus interventions with young people alone

Also, we detected no effects in subgroup analysis including universal studies only (SMD $-0.44,95 \% \mathrm{Cl}-1.08$ to 0.20 ; studies $=3$; participants $=5351 ; I^{2}=99 \%$; Analysis 2.4 ).

Insufficient numbers of studies precluded other subgroup analyses.

We did not pool two studies due to lack of experimental isolation of the parent component of the intervention (Spirito 2015), or to heterogeneity of the comparison group (Stevens 2002). Spirito 2015 compared a selective parent-focused intervention versus a brief psychoeducational intervention for adolescents in which parents attended the sessions; both conditions involved booster mailouts for parents over a six-month period. This study reported a significant interaction of the study condition by time on adolescent report of refusal to drink alcohol $\left(F(1,56)=7.05, P<0.05\right.$, partial $\eta^{2}$ $=0.11$ ), such that adolescents in the family check-up intervention condition reported significant increases in alcohol refusal from baseline to six months when compared to adolescents in the psychoeducational condition, who reported significant decreases in alcohol refusal. Stevens 2002 compared a universal family-based alcohol and tobacco programme delivered through paediatric primary care versus a family-based safety (gun, seatbelt, bicycle helmet) programme, also delivered through paediatric primary care. Researchers reported no significant intervention effects.

\section{Frequency of alcohol use}

Comparative effectiveness trials measuring alcohol use frequency reported no effects overall. The SMD slightly favoured a decreased frequency of use, but variability and heterogeneity were substantial (SMD $-0.16,95 \% \mathrm{Cl}-0.42$ to 0.09 ; studies $=4$; participants $=915 ; \mathrm{I}^{2}$ $=73 \%$; Analysis 2.2 ; very low-quality evidence). Sensitivity analysis with the three studies classified as low risk of bias remained consistent (SMD $-0.21,95 \% \mathrm{Cl}-0.50$ to 0.08 ; studies $=3$; participants $=432 ; I^{2}=80 \%$; Analysis 2.3).

\section{Subgroup analyses}

Two universal studies measuring alcohol use frequency were pooled in a subgroup analysis, again revealing an absence of intervention effect (SMD $-0.30,95 \% \mathrm{Cl}-0.68$ to 0.07 ; studies $=2$; participants $=596 ; \mathrm{I}^{2}=84 \% ;$ Analysis 2.6$)$ versus interventions with young people alone (Schinke 2004; Werch 2008). Similarly, twoindicated studies measuring alcohol use frequency showed no overall effect (SMD 0.01, $95 \% \mathrm{Cl}-0.21$ to 0.23 ; studies $=2 ;$ participants $=319 ; 1^{2}=0 \%$; Analysis 2.7) (Spirito 2011; Winters 2012). These studies compared interventions targeted towards adolescents only, such as a CD-ROM programme (Schinke 2004), postcards containing health promotion messages (Werch 2008), individual motivational interviewing (Spirito 2011), and adolescent brief intervention (Winters 2012), with interventions which included these adolescent components along with parent involvement.

Results among two of the three studies that could not be pooled in this category remained consistent with the overall lack of intervention effects. Perry 2003 conducted linear growth curve analyses and reported that the universal DARE plus program enhanced the DARE curriculum, with both girls and boys in the DARE Plus group less likely to increase their alcohol use over time. We did not pool two studies due to heterogeneity and the lack of experimental isolation of the parent component of the intervention. Spirito 2017 compared an indicated parentfocused intervention versus a brief psychoeducational intervention for adolescents in which parents attended the sessions, and both conditions involved booster mail-outs for parents over a six-month period. This study reported no treatment effect by condition. Similarly, in their indicated intervention programme, Liddle 2008 compared multi-dimensional family therapy versus cognitive-behavioural therapy delivered to adolescents but with parents involved in the first two sessions and in signing a treatment contract. No differential treatment effects were reported for alcohol use between groups. 


\section{Volume of alcohol use}

No comparative effectiveness trial reporting on the volume of alcohol use could be pooled in meta-analysis.

\section{Secondary outcomes}

Several studies reported secondary outcomes in addition to alcohol use outcomes, but we did not pool these due to the heterogeneity of measures.

\section{Universal interventions}

One study reported on parental supply of alcohol at home following a universal intervention based on the Orebro programme and noted a statistically significant programme effect compared to control at 12 months' and 30 months' follow-up using intention-totreat analysis $(\mathrm{P}=0.03$ and 0.01 , respectively) and using multiple imputation at 30 months only $(P=0.02)$ (Bodin 2011).

Another study measured alcohol misuse following a universal intervention involving three family sessions at home (LovelandCherry 1999). Researchers used a composite of eight items and found that prior drinkers in the intervention condition reported less alcohol misuse and a sharper decline in alcohol problems than prior drinkers in the control group, but those who were not prior drinkers showed a slight increase in alcohol misuse, with intervention group participants only reporting minimally lower rates of misuse than control group participants. Effects of the intervention condition by time interaction were significant for alcohol misuse $(P<0.01)$. We did not include this outcome in the meta-analysis as the study also reported a quantity-frequency composite, which we used in the alcohol use volume category.

Schinke 2004 measured family involvement including monitoring and the number of times parents had spoken to adolescents about not drinking following a universal CD-ROM-based programme. Study authors reported higher family involvement scores at two and three years' follow-up among the intervention group versus the control group.

\section{Selective interventions}

Prado 2012 reported on a diagnosis of alcohol dependence using adolescent reports on the Diagnostic Interview Schedule for Children (DISC) predictive scales following a selective intervention based on family and group counselling sessions. This study reported a decrease in the percentage of adolescents with dependence in the intervention group and an increase in the control group. In growth curve analysis, the difference over time between groups was significant $(b=-1.16, P=0.02, P=0.93)$.

\section{Indicated interventions}

Two studies of indicated interventions reported relevant secondary outcomes. Winters 2012 used an Adolescent Diagnostic Interview (ADI) following a brief intervention-based programme to measure symptoms of both alcohol abuse and dependency in the prior 90 days, and reported effect sizes of 2.0 and 2.1, respectively, with higher rates of 'absence' of these symptoms in the intervention group than in the control group ( $\mathrm{P}<0.01$ for both).

Arnaud 2016 also measured alcohol problems using the brief Rutgers Alcohol Problems Index following their brief motivational interviewing programme. Study authors report that although alcohol problems were reduced over time in both intervention and control groups, between-group differences were not significant.

\section{DISCUSSION}

\section{Summary of main results}

This update and expansion of a previous review - Foxcroft 2011a - identified a large number of randomised controlled trials (RCTs) and cluster-randomised controlled trials (C-RCTs) investigating family-based interventions aimed at reducing alcohol use in young people. We have included a total of 46 studies in this review.

Overall this comprehensive systematic review and meta-analysis found little evidence to show that universal, selective, and indicated interventions with parents or families are effective in reducing measures of adolescent alcohol consumption (prevalence, frequency, and volume of alcohol consumption) when compared to no intervention or standard care. Some evidence suggests that under certain circumstances, universal and selective/ indicated family-based interventions had small, differential effects on measures of adolescent alcohol use; however, in light of the number of analyses conducted, the variation in effects observed, the high risk of bias assessed across studies, and the level of heterogeneity noted, the overall interpretation of outcomes indicates no effect.

Analysis of studies comparing the effects of a family-based intervention versus no intervention or usual care control on the prevalence of alcohol use showed no impact. Subgroup analyses examining the effects of selective and indicated interventions, targeting at risk or already using groups of adolescents, showed no clear effects on alcohol prevalence. Similarly, results show no discernible impact of studies comparing a family-based intervention as part of another intervention versus the other intervention alone. Studies that could not be pooled reported mixed findings, with three studies reporting no intervention effects (Furr-Holden 2004; Prado 2012; Spirito 2015), and three reporting some statistically significant positive effects (Cordova 2012; O'Donnell 2010; Stevens 2002).

Overall we found no clear evidence of intervention effects on the frequency of alcohol use, but the patterns observed in subgroup analyses warrant further consideration. Meta-analysis of all studies measuring the effects of family/parent interventions compared to control on the frequency of alcohol use also failed to show a reduction in this outcome. Subgroup analyses suggest that universal interventions targeting low-risk groups have the potential for harmful effects on this outcome, and selective and indicated interventions are more likely to favour a reduction in the frequency of use. Potential for effectiveness is also observed in subgroup analyses of studies that focus on ethnic minority groups. A small positive effect was found in meta-analysis of comparative effectiveness trials. Of the seven studies that could not be pooled, four reported some positive effects of their interventions on the frequency of alcohol use (Dembo 2001; Milburn 2012; Perry 2003; Wolchik 2002), and three reported no differential effects between groups (Estrada 2017; Liddle 2008; Spirito 2017).

It is possible that the lower-risk groups targeted in universal interventions and represented in ethnic majority populations fail to demonstrate positive effects due to a lower population prevalence of alcohol consumption, making differences between groups more 
difficult to detect in inadequately powered studies. Conversely, higher-risk groups such as those targeted in selective and indicated interventions have higher rates of consumption, making detection of differences more achievable. It is also possible that consumption frequency is a more relevant and powerful measure of effect in selective and indicated studies, with reduction in consumption frequency a more realistic expectation for participants who are at risk or are already using. Among the populations targeted in universal interventions, it is expected that a natural increase in the prevalence of drinking over time would occur in both intervention and control groups. The observed patterns raise a question as to whether highlighting the topic of drinking in the intervention programmes might actually stimulate this increase more in intervention groups than in control groups.

Differing patterns were also observed in meta-analysis of studies measuring intervention effects on alcohol use volume. In subgroup analyses, the interventions classified as universal had a small intervention-favouring effect on the volume of alcohol consumed, as did the subgroup of studies involving ethnic minority participants and those involving females only. The two pooled studies trialling selective or indicated interventions showed an overall negative (potentially harmful) effect on alcohol consumption volume. Comparative effectiveness trials measuring alcohol consumption volume could not be pooled. Three studies that could not be pooled reported positive intervention effects on alcohol use volume (Fosco 2013; Schinke 2009a; Stormshak 2011). These patterns should be interpreted with caution, however, with small numbers of studies contributing to each of these findings and to subgroup analyses of studies involving females only, including two studies conducted by the same group (Schinke 2009c; Schinke 2011). A broader range of independent studies is needed to contribute to these analyses and to confirm the borderline effects observed here.

Notwithstanding these limitations, the patterns observed warrant further investigation. The patterns are somewhat in conflict with those reported for alcohol frequency above, suggesting that there may be important differences between these constructs. It is possible that in a similar way to adult consumption, adolescent alcohol consumption varies as a product of both of these measures, with less frequent but higher-volume consumption representing greater risk than the reverse.

Effects on secondary outcomes could not be pooled due to heterogeneity, and results were mixed. Bodin reported positive intervention effects on parental supply of alcohol both 12 and 30 months after their trial of the Orebro prevention programme. One universal study measured alcohol misuse outcomes (LovelandCherry 1999), with positive intervention effects reported among participants who were prior drinkers at baseline but negative effects reported among those who were not.

One selective study - Prado 2012 - and one indicated study Winters 2012 - measured alcohol abuse and dependence, with both reporting lower rates of these diagnoses among intervention groups, although Winters 2012 reported significant differences only between their brief intervention with adolescents and parents versus control - not versus the adolescent brief intervention only. One indicated programme also measured alcohol problems and found no differences between groups (Arnaud 2016).
Across all types of interventions, programmes consistently focused on parent-child relationships and communication and on promoting positive parenting approaches in their effort to reduce alcohol or other substance use. No clear differences between the interventions used in effective and ineffective trials are discernible. Findings show a pattern of increased programme intensity in selective and indicated interventions, which is worth considering. Kuntsche 2016 suggested that increased programme intensity of parent-based interventions is associated with increased effectiveness, but noted that high-intensity effective interventions are more commonly trialled in high-risk populations. It is possible that in selective and indicated interventions, and with older adolescent groups, high-intensity interventions are more appropriate, but that universal interventions are more likely to be effective when they require less intensive or face-to-face parental involvement. Within the universal interventions, at an individual study level, a pattern exists whereby effective studies (e.g. Bodin 2011, Schinke 2009b, Schinke 2009c) require less parental face-toface involvement than those that are ineffective (e.g. Linakis 2013, Spoth 2002). Subgroup analysis including only the universal studies regarded as lower intensity (defined as self-directed, computermediated, or including face-to-face contact that coincides with other parental commitments at school) approaches significance but remains highly heterogeneous (not shown).

The selective and indicated interventions tended to be aimed towards older adolescents, with approximate average ages for universal, selective, and indicated studies of 12,13 , and 15.5 years, respectively. Two studies included participant groups with average age less than 10 years (Furr-Holden 2004; LovelandCherry 1999). One of these - Furr-Holden 2004 - was not included in the meta-analysis for other reasons, but when a subgroup analysis was performed excluding Loveland-Cherry 1999, the results did not differ (not reported). These patterns of increasing age and increasing programme intensity for increasing risk level of participants are likely to reflect an appropriate application of the proportionate universalism concept (Marmot 2010).

\section{Overall completeness and applicability of evidence}

Forty-six trials with 39,822 randomised participants met the inclusion criteria for this review. Most trials $(n=29)$ were reported from the United States; others were conducted primarily in Europe (Netherlands, Germany, Poland, Sweden); and only one study - Reddy 2002 - was conducted in a developing nation (India). This fact limits the external validity of the evidence and the generalisability of results to developing nations. Six trials were specific to females only, reporting gender-specific interventions, but subgroup analysis by gender was limited, as these studies did not report comparable outcomes. Also four of these six studies were linked to the same programme and were led by the same primary researcher, meaning that additional, independent studies are required to strengthen evidence associated with the impact of family-based interventions on girls' alcohol use. The age of adolescents targeted in the included studies largely represents children of secondary school age before or during the time when initiation to alcohol commonly begins. This review excluded studies targeting adolescents transitioning to college, as the role of parents is different in this context, and advice has to account for students likely to be moving away from home and into college housing settings. Thus the included studies are more homogenous in their limitation to adolescents of average age less than 18 years. 
The included study settings and family-based programmes were consistent with those found in prevention settings in the United States and Europe.

\section{Quality of the evidence}

Despite improvements over time in the methodological quality of trials of alcohol misuse prevention for young people (Foxcroft 2011a), there remain important methodological limitations and reporting problems. Overall, 17 of the included studies were regarded as high risk for the purpose of sensitivity analysis. Although these analyses did not lead to discernible differences in study results, it is important to note the common sources of bias across included studies, with most studies rated as having high risk of bias for blinding of participants and personnel, and for blinding of outcome assessment, due to the nature of the interventions and the study design. Most studies provided insufficient detail to enable assessment of allocation (selection bias) and reporting bias.
We used the GRADE approach to assess the quality of evidence for the primary outcome of alcohol use, conducting assessments separately for each outcome and comparison type. See Summary of findings for the main comparison and Summary of findings 2 . The quality of evidence for a reduction in alcohol use ranged from low to very low.

We assessed the quality of evidence for trials comparing parent/ family interventions versus no intervention/standard care in reducing the prevalence of alcohol use as low due to downgrading on account of risk of bias and unexplained heterogeneity. Downgrading for risk of bias (one level) was due to unclear risk of bias ratings in several domains, and downgrading for heterogeneity (one level) was due to moderate heterogeneity that was explained only partially in subgroup analyses. We did not downgrade quality for publication bias due to symmetry in funnel plots (Figure 4; Figure 5; Figure 6).

Figure 4. Funnel plot of comparison: 1 Parent/family intervention vs control_2, outcome: 1.1 Alcohol use_Prevalence.

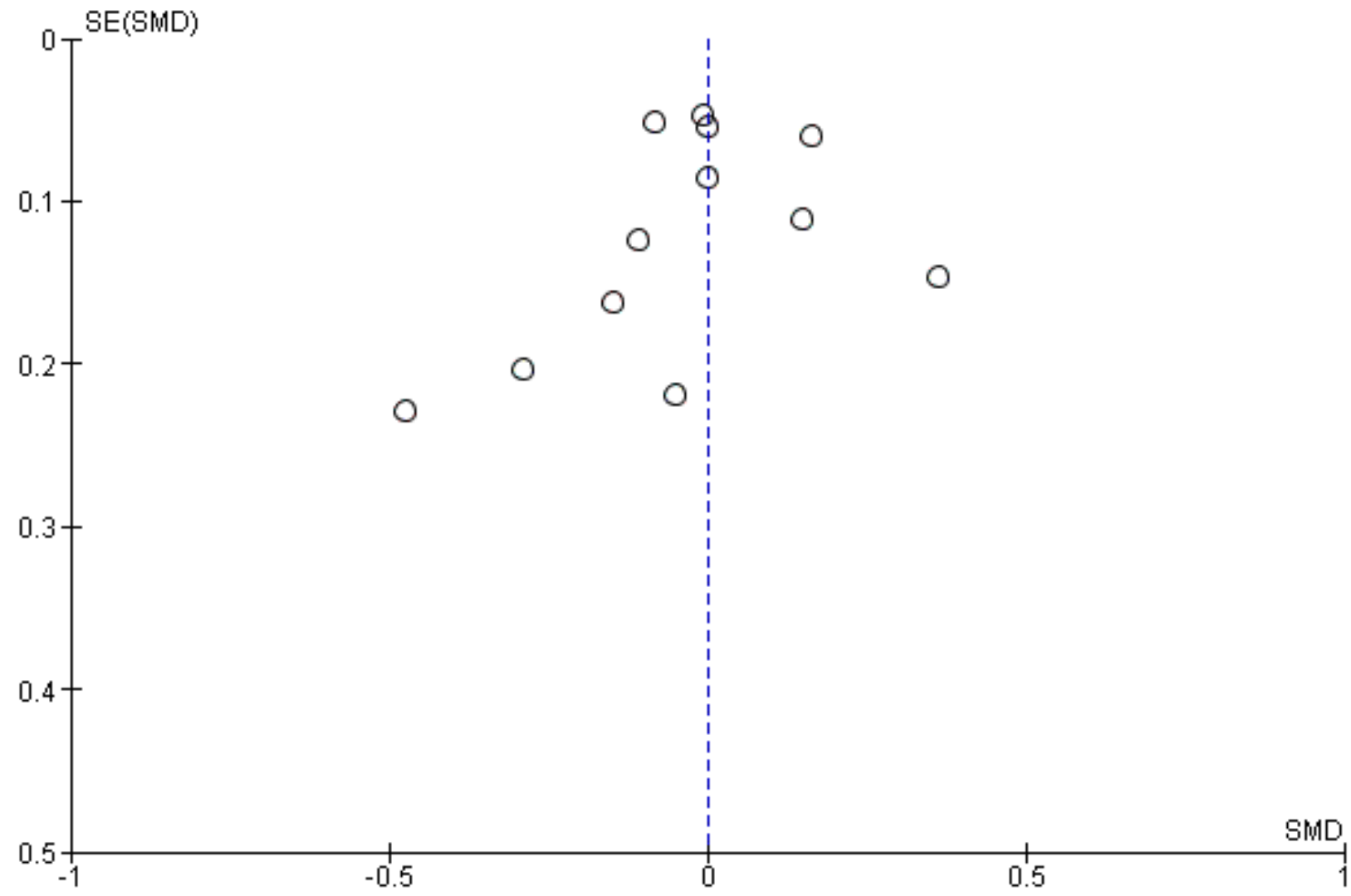


Figure 5. Funnel plot of comparison: 1 Parent/family intervention vs control_2, outcome: 1.5 Subgroup_universal_Alcohol use_Prevalence.

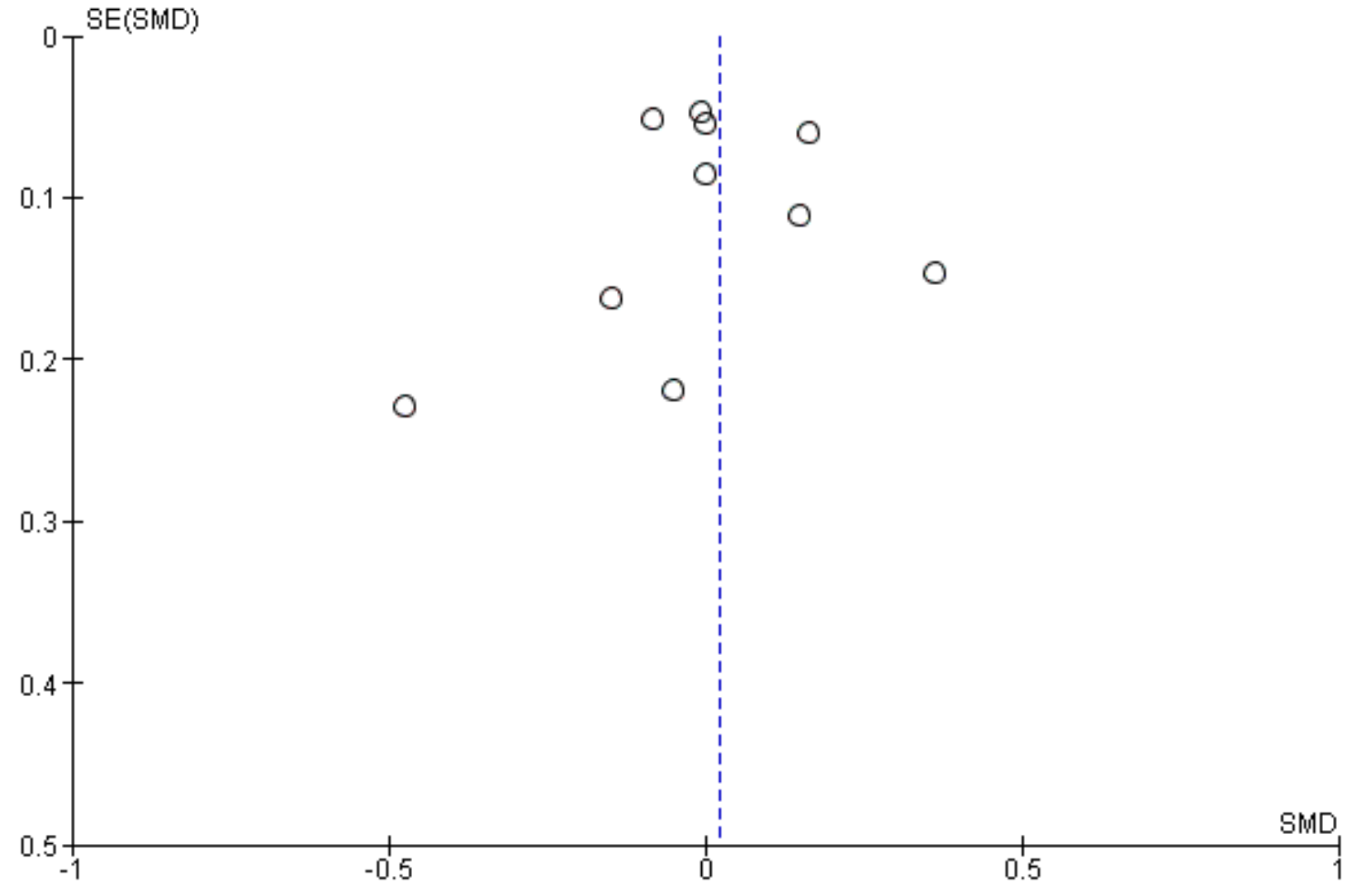


Figure 6. Funnel plot of comparison: 1 Parent/family intervention vs control_2, outcome: 1.8 Subgroup_>12 months_Alcohol use_Prevalence.

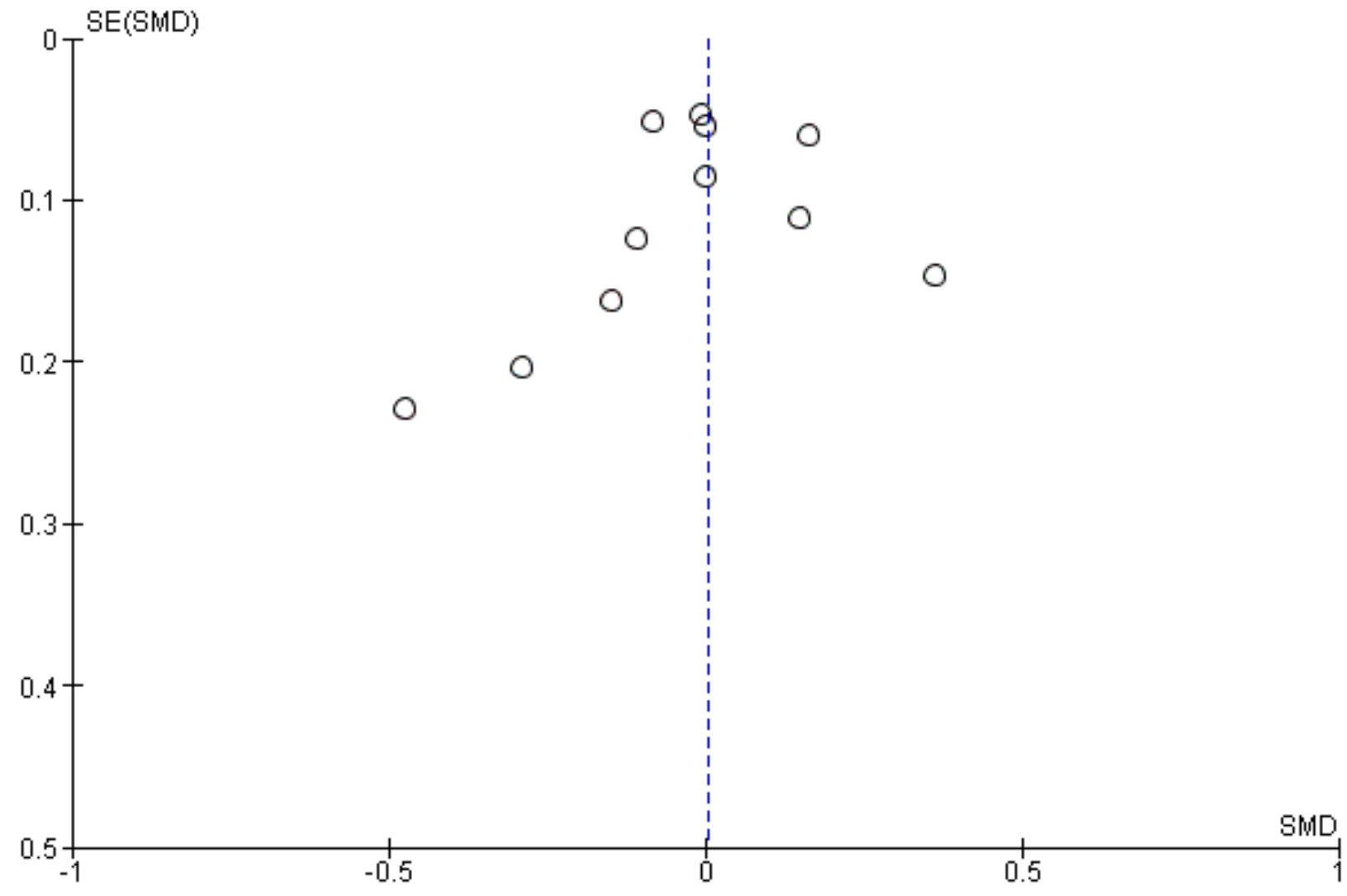

We assessed the quality of evidence for trials comparing parent/ family interventions versus no intervention/standard care in reducing the frequency of alcohol use as very low due to downgrading on account of risk of bias, heterogeneity, and imprecision. Downgrading one level for risk of bias was due to high or unclear risk of bias ratings in several domains. Dowgrading two levels for heterogeneity was related to high heterogeneity that was not explained in subgroup analyses. Downgrading one level for imprecision of results was a result of a wide confidence interval that crosses both -0.5 and zero, meaning that the true effect could be either beneficial or harmful.

We assessed the quality of evidence for trials comparing parent/ family interventions versus no intervention/standard care in reducing the volume of alcohol use as low due to downgrading one level on account of high or unclear risk of bias ratings in several domains, and downgrading one level due to a high probability of publication bias. We assessed risk of publication bias as high due to the small proportion of included studies that could be pooled in the meta-analysis.

For comparative effectiveness trials, we assessed the quality of evidence for measuring alcohol use prevalence as very low due to downgrading on account of high or unclear risk of bias ratings in several domains (one level) and imprecision of results (one level) with a wide confidence interval that crosses both -0.5 and zero, meaning that the true effect could be either beneficial or harmful. Also, we downgraded this evidence two levels on account of high heterogeneity that was not explained in subgroup analyses. We assessed comparative effectiveness trials measuring alcohol use frequencies as providing very lowquality evidence due to downgrading on account of risk of bias (one level), heterogeneity (one level), and imprecision (one level). Downgrading for imprecision of results was a result of a wide confidence interval that crosses both -0.5 and zero and a relatively small sample size, meaning that the true effect could be either beneficial or harmful.

\section{Potential biases in the review process}

This review used a comprehensive, rigorous methodology and a broad search strategy, and pairs of independent review authors conducted screening, data extraction, and appraisal of risk of bias. Furthermore, review authors did not restrict publications on the basis of language. Therefore it is unlikely that relevant trials were missed. Further information was sought for five of the included studies by contacting the lead or corresponding author; information was received from two of these study authors.

\section{Agreements and disagreements with other studies or reviews}

The effort to update and expand the Foxcroft 2011a review led to inclusion of an additional 34 studies and enabled meta-analytical pooling of data. This analysis has shifted the overall result from one of promising positive effects to one of essentially no effect. It is important to consider the substantial heterogeneity and 
potential biases in the included studies in any interpretation of current results. These findings are also somewhat in conflict with those reported by Smit 2008 in a review conducted before the Foxcroft Cochrane review, as well as in a recent update by Kuntsche 2016. Each of these reviews points towards small but positive effects of universal, family-based prevention interventions. The addition of 12 selective and 7 indicated intervention studies to the present review demonstrates the likely differential effectiveness of intervention programmes according to the target population, with positive intervention effects less clear across these studies. The breakdown of outcome measures provided in the present review could also contribute to differences in findings, with effects seen to differ across measures of prevalence, frequency, and volume of use.

Kuntsche 2016 conducted a systematic literature review of parent-based interventions for preventing or reducing adolescent substance use, including 39 publications dealing with 13 intervention programmes. Five of the included programmes are also included in the present review, but Kuntsche 2016 included only studies published in the 12 years since publication of a previous review (Petrie 2007), studies involving adolescents of mean age 10 to 18 years (excluding studies involving younger children), and studies reporting as an outcome the initiation of alcohol, tobacco, or cannabis. This review also included some quasi-experimental studies with pre-post evaluation design. Review authors concluded that support for parents in improving parent-child communication and monitoring their offspring's activities while providing strict rules against underage substance use can help curb adolescent substance use. The review authors noted several limitations and gaps in their review that require further investigation. These questions relate in particular to programme intensity, with a trend observed whereby the intensity of a programme has a direct impact on its effectiveness, and where more intense programmes are most commonly trialled in older, high-risk (ethnic minority) populations. As also identified in the present review, questions remain regarding intensity in relation to types of programmes (universal, selective, indicated) and to ages and types of participants. Further, this review reports narratively on the findings of included studies grouped broadly as investigating alcohol use, drunkenness, or alcohol problems.

Vermeulen-Smit 2015 reviewed a similar set of studies, including 39 articles reporting on 18 different programmes but describing outcomes related to illicit substances. These review authors included only programmes in which at least half of the intervention was aimed at parents and reported that such programmes had a small favourable effect on the initiation and frequency of marijuana use but provided no clear evidence of an impact on other illicit drug use.

\section{AUTHORS' CONCLUSIONS}

\section{Implications for practice}

Current evidence suggests that family-based prevention programmes alone are not likely to be effective in reducing adolescent alcohol use. Further, consideration should be given to the relationship between alcohol consumption frequency and volume, with the potential for practically significant public health benefits of a reduction in alcohol consumption volume. Conversely though, the public health significance of a reduction in consumption frequency could be outweighed by a negative impact on or increase in the volume of consumption. Funders of prevention programmes should carefully consider the target group and should ensure that targeted outcomes and programme intensity appropriately match the age and risk level of the group. The timing and approach of universal prevention programmes should be carefully considered to avoid any potential for these interventions to inadvertently stimulate alcohol use in young people who were not already drinking.

\section{Implications for research}

Given the small numbers of studies included in each of these subgroup analyses, the variability in effect sizes, and the high level of unexplained heterogeneity, it is recommended that further evaluation should be conducted, with particular attention paid to the content and context of programme delivery and the differential effects on key outcomes. Questions remain about the impact of programme intensity and context (target population and mode of delivery) on measured effects. Future research should investigate the differential effects of various intervention types on different outcome measures to better understand the implications of effects on alcohol use prevalence, frequency, and volume. Further evaluation of previous and future studies with the view toward identifying the components most likely to be appropriate and effective in universal, selective, and indicated interventions is recommended. Further improvement is also required in study design, analysis, and reporting to minimise the risk of bias and improve the quality of evidence generated.

\section{ACKNOWLEDGEMENTS}

We acknowledge the work of previous Cochrane Review authors, whose efforts have contributed to the present protocol and review; in particular, David Foxcroft and Alexander Tsertsvadze. We also acknowledge the funding and intellectual contributions made by the Australian Drug Foundation (ADF). 


\section{RE F E R E N C E S}

\section{References to studies included in this review}

Arnaud 2016 \{published data only\}

Arnaud N, Diestelkamp S, Wartberg L, Sack PM, Daubmann A, Thomasius R. Short- to midterm effectiveness of a brief motivational intervention to reduce alcohol use and related problems for alcohol intoxicated children and adolescents in pediatric emergency departments: a randomized controlled trial. Academic Emergency Medicine 2017;24(2):186-200.

\section{Baldus 2016 \{published data only\}}

Baldus C, Thomsen M, Sack P-M, Broning S, Arnaud N, Daubmann A, et al. Evaluation of a German version of the strengthening families programme 10-14: a randomised controlled trial. European Journal of Public Health 2016;26(6):953-9.

\section{Bauman 2002 \{published data only\}}

*Bauman KE, Ennett ST, Foshee VA, Pemberton M, King TS, Koch GG. Influence of a family program on adolescent smoking and drinking prevalence. Prevention Science 2002;3(1):35-42.

Bauman KE, Ennett ST, Foshee VA, Pemberton M, King TS, Koch GG. Influence of a family-directed program on adolescent cigarette and alcohol cessation. Prevention Science 2000;1(4):227-37.

Bauman KE, Foshee VA, Ennett ST, Pemberton M, Hicks KA, King TS, et al. The influence of a family program on adolescent tobacco and alcohol use. American Journal of Public Health 2001;91(4):604-10.

\section{Bodin 2011 \{published data only\}}

Bodin MC, Strandberg AK. The Orebro prevention programme revisited: a cluster-randomized effectiveness trial of programme effects on youth drinking. Addiction 2011;106(12):2134-43.

\section{Brody 2006 \{published data only\}}

Brody GH, Chen YF, Kogan SM, Murry VM, Brown AC. Longterm effects of the Strong African American Families Program on youth's alcohol use. Journal of Consulting and Clinical Psychology 2010;78(2):281-5.

* Brody GH, Murry VM, Kogan SM, Gerrard M, Gibbons FX, Molgaard V, et al. The Strong African American Families Program: a cluster-randomised prevention trial of long-term effects and a mediational model. Journal of Consulting and Clinical Psychology 2006;74(2):356-66.

Gerrard M, Gibbons FX, Brody GH, Murry VM, Cleveland MJ, Wills TA. A theory-based dual-focus alcohol intervention for preadolescents: the Strong African American Families Program. Psychology of Addictive Behaviors 2006;20(2):185-95.

\section{Catalano 1999 \{published data only\}}

Catalano RF, Gainey RR, Fleming CB, Haggerty KP, Johnson NO. An experimental intervention with families of substance abusers: one-year follow-up of the Focus On Families project. Addiction 1999;94(2):241-54.
Cordova 2012 \{published data only\}

* Cordova D, Huang S, Pantin H, Prado G. Do the effects of a family intervention on alcohol and drug use vary by nativity status?. Psychology of Addictive Behaviors 2012;26(3):655-60.

Pantin H, Prado G, Lopez B, Huang S, Tapia MI, Schwartz SJ, et al. A randomized controlled trial of Familias Unidas for Hispanic adolescents with behavior problems. Psychosomatic Medicine 2009;71(9):987.

\section{Dembo 2001 \{published data only\}}

Dembo R, Schmeidler J, Seeberger W, Shemwell M, Rollie M, Pacheco K, et al. Long-term impact of a Family Empowerment Intervention on juvenile offender psychosocial functioning. Journal of Offender Rehabilitation 2001;33(1):59-109.

* Dembo R, Wothke W, Livingston S, Schmeidler J. The impact of a family empowerment intervention on juvenile offender heavy drinking: a latent growth model analysis. Substance Use \& Misuse 2002;37(11):1359-90.

\section{Estrada 2017 \{published data only\}}

Estrada Y, Lee TK, Huang S, Tapia MI, Velazquez MR, Martinez MJ, et al. Parent-centered prevention of risky behaviors among Hispanic youths in Florida. American Journal of Public Health 2017;107(4):607-13.

Fang 2010 \{published data only\}

Fang L, Schinke SP. Mediation effects of a culturally generic substance use prevention program for Asian American adolescents. Asian American Journal of Psychology 2014;5(2):116-25

* Fang L, Schinke SP. Two-year outcomes of a randomized, family-based substance use prevention trial for Asian American adolescent girls. Psychology of Addictive Behaviors 2013;27(3):788-98

Fang L, Schinke SP, Cole KC. Preventing substance use among early Asian-American adolescent girls: initial evaluation of a web-based, mother-daughter program. Journal of Adolescent Health 2010;47(5):529-32.

\section{Fosco 2013 \{published data only\}}

Fosco GM, Frank JL, Stormshak EA, Dishion TJ. Opening the "Black Box": family check-up intervention effects on selfregulation that prevents growth in problem behavior and substance use. Journal of School Psychology 2013;51(4):455-68.

\section{Foxcroft 2017 \{published data only\}}

Foxcroft DR, Callen H, Davies EL, Okulicz-Kozaryn K. Effectiveness of the Strengthening Families Programme 10-14 in Poland: cluster randomized controlled trial. European Journal of Public Health 2017;27:494-500.

\section{Furr-Holden 2004 \{published data only\}}

Furr-Holden CD, lalongo NS, Anthony JC, Petras H, Kellam SG. Developmentally inspired drug prevention: middle school outcomes in a school-based randomized prevention trial. Drug \& Alcohol Dependence 2004;73(2):149-58. 
Haggerty 2007 \{published data only\}

Haggerty KP, Skinner M, Fleming CB, Gainey RR, Catalano RF. Long-term effects of the Focus on Families project on substance use disorders among children of parents in methadone treatment. Addiction 2008;103(12):2008-16.

* Haggerty KP, Skinner ML, MacKenzie EP, Catalano RF. A randomised trial of Parents Who Care: effects on key outcomes at 24-month follow-up. Prevention Science 2007;8(4):249-60.

\section{Koning 2009 \{published data only\}}

Koning IM, Lugtig P, Vollebergh WA. Differential effects of baseline drinking status: effects of an alcohol prevention program targeting students and/or parents (PAS) among weekly drinking students. Journal of Substance Abuse Treatment 2014;46(4):522-7.

Koning IM, Vollebergh WA, Smit F, Verdurmen JE, van den Eijnden RJ, ter Bogt T, et al. Preventing heavy alcohol use in adolescents (PAS): cluster randomised trial of a parent and student intervention offered separately and simultaneously. Addiction 2009;104(10):1669-78.

Koning IM, van den Eijnden RJ, Verdurmen JE, Engels RC, Vollebergh WA. A cluster randomized trial on the effects of a parent and student intervention on alcohol use in adolescents four years after baseline; no evidence of catching-up behavior. Addictive Behaviors 2013;38(4):2032-9.

* Koning IM, van den Eijnden RJ, Verdurmen JE, Engels RC, Vollebergh WA. Long-term effects of a parent and student intervention on alcohol use in adolescents: a cluster randomized controlled trial. American Journal of Preventive Medicine 2011;40(5):541-7.

Koning IM, van den Eijnden RJJM, Engels RCME, Verdurmen JEE, Vollebergh WAM. Why target early adolescents and parents in alcohol use? The mediating effects of self-control, rules, and attitudes about alcohol use. Addiction 2010;106:538-46. [DOI: 10.1111/j.1360-0443.2010.03198.x]

\section{Liddle 2008 \{published data only\}}

Liddle HA, Dakof GA, Turner RM, Henderson CE, Greenbaum PE. Treating adolescent drug abuse: a randomized trial comparing multidimensional family therapy and cognitive behavior therapy. Addiction 2008;103(10):1660-70.

\section{Linakis 2013 \{published data only\}}

Linakis JG, Bromberg J, Baird J, Nirenberg TD, Chun TH, Mello MJ, et al. Feasibility and acceptability of a pediatric emergency department alcohol prevention intervention for young adolescents. Pediatric Emergency Care 2013;29(11):1180-8

\section{Loveland-Cherry 1999 \{published data only\}}

* Loveland-Cherry CJ, Ross LT, Kaufman SR. Effects of a homebased family intervention on adolescent alcohol use and misuse. Journal of Studies on Alcohol 1999;Suppl 13:94-102.

\section{Mares 2016 \{published data only\}}

Mares SHW, Lichtwarck-Aschoff A, Verdurmen J, Schulten I, Engels RCME. Home-based alcohol prevention program for parents and children: a randomized controlled trial. Preventive Medicine 2016;88:224-9.

\section{Mason 2012 \{published data only\}}

Mason W, Haggerty KP, Fleming AP, Casey-Goldstein M. Family intervention to prevent depression and substance use among adolescents of depressed parents. Journal of Child and Family Studies 2012;21(6):891-905.

Milburn 2012 \{published data only\}

Milburn NG, Iribarren FJ, Rice E, Lightfoot M, Solorio R, Rotheram-Borus MJ, et al. A family intervention to reduce sexual risk behavior, substance use, and delinquency among newly homeless youth. Journal of Adolescent Health 2012;50(4):358-64.

\section{O'Donnell 2010 \{published data only\}}

* O’Donnell L, Myint-U A, Duran R, Stueve A. Especially for Daughters: parent education to address alcohol and sexrelated risk taking among urban young adolescent girls. Health Promotion Practice 2010;11(Suppl 1):70S-78S.

\section{Perry 2003 \{published data only\}}

Perry CL, Komro KA, Veblen-Mortenson S, Bosma LM, Farbakhsh $\mathrm{K}$, Munson KA, et al. A randomized controlled trial of the middle and junior high school D.A.R.E. and D.A.R.E. Plus programs. Archives of Pediatrics \& Adolescent Medicine 2003;157(2):178-84.

\section{Prado 2012 \{published data only\}}

Prado G, Cordova D, Huang S, Estrada Y, Rosen A, Bacio GA, et al. The efficacy of Familias Unidas on drug and alcohol outcomes for Hispanic delinquent youth: main effects and interaction effects by parental stress and social support. Drug \& Alcohol Dependence 2012;125 Suppl 1:S18-25.

\section{Reddy 2002 \{published data only\}}

Reddy KS, Arora M, Perry CL, Nair B, Kohli A, Lytle LA, et al. Tobacco and alcohol use outcomes of a school-based intervention in New Delhi. American Journal of Health Behavior 2002;26(3):173-81.

\section{Riesch 2012 \{published data only\}}

Riesch SK, Brown RL, Anderson LS, Wang K, Canty-Mitchell J, Johnson DL. Strengthening Families Program (10-14): effects on the family environment. Western Journal of Nursing Research 2012;34(3):340-76

\section{Schinke 2004 \{published data only\}}

* Schinke SP, Schwinn TM, Di Noia J, Cole KC. Reducing the risks of alcohol use among urban youth: three-year effects of a computer-based intervention with and without parent involvement. Journal of Studies on Alcohol 2004;65(4):443-9.

Schinke SP, Schwinn TM, Fang L. Longitudinal outcomes of an alcohol abuse prevention program for urban adolescents. Journal of Adolescent Health 2010;46(5):451-7.

Schwinn TM, Schinke SP. Preventing alcohol use among late adolescent urban youth: 6-year results from a computer-based intervention. Journal of Studies on Alcohol 2010;71(4):535-8. 
Schinke 2009a \{published data only\}

* Schinke SP, Cole KC, Fang L. Gender-specific intervention to reduce underage drinking among early adolescent girls: a test of a computer-mediated, mother-daughter program. Alcohol and Drugs 2009;70(1):70-7.

\section{Schinke 2009b \{published data only\}}

* Schinke SP, Fang L, Cole KC. Computer-delivered, parentinvolvement intervention to prevent substance use among adolescent girls. Preventive Medicine 2009;49(5):429-35

\section{Schinke 2009c \{published data only\}}

* Schinke SP, Fang L, Cole KC. Preventing substance use among adolescent girls: 1-year outcomes of a computerized, motherdaughter program. Addictive Behaviors 2009;34(12):1060-4.

\section{Schinke 2011 \{published data only\}}

Schinke SP, Fang L, Cole KC, Cohen-Cutler S. Preventing substance use among Black and Hispanic adolescent girls: results from a computer-delivered, motherdaughter intervention approach. Substance Use \& Misuse 2011;46(1):35-45.

\section{Skarstrand 2014 \{published data only\}}

Skarstrand E, Sundell K, Andreasson S. Evaluation of a Swedish version of the Strengthening Families Programme. European Journal of Public Health 2014;24(4):578-84.

\section{Spirito 2011 \{published data only\}}

Spirito A, Sindelar-Manning H, Colby SM, Barnett NP, Lewander W, Rohsenow DJ, et al. Individual and family motivational interventions for alcohol-positive adolescents treated in an emergency department: results of a randomized clinical trial. Archives of Pediatrics \& Adolescent Medicine 2011;165(3):269-74.

\section{Spirito 2015 \{published data only\}}

Spirito A, Hernandez L, Cancilliere MK, Graves H, Barnett N. Improving parenting and parent-adolescent communication to delay or prevent the onset of alcohol and drug use in young adolescents with emotional/behavioral disorders: a pilot trial. Journal of Child \& Adolescent Substance Abuse 2015;24(5):308-22.

\section{Spirito 2017 \{published data only (unpublished sought but not} used)\}

Spirito A, Hernandez L, Marceau K, Cancilliere MK, Barnett NP, Graves HR, et al. Effects of a brief, parent-focused intervention for substance using adolescents and their sibling. Journal of Substance Abuse Treatment 2017;77:156-65.

\section{Spoth 1999a \{published data only\}}

Guyll M, Spoth RL, Chao W, Wickrama KA, Russell D. Familyfocused preventive interventions: evaluating parental risk moderation of substance use trajectories. Journal of Family Psychology 2004;18(2):293-301.

Mason WA, Kosterman R, Haggerty KP, Hawkins JD, Redmond C, Spoth RL, et al. Gender moderation and social developmental mediation of the effect of a family-focused substance use preventive intervention on young adult alcohol abuse. Addictive Behaviors 2009;34(6-7):599-605.

Park J, Kosterman R, Hawkins JD, Haggerty KP, Duncan TE, Duncan SC, et al. Effects of the "Preparing for the Drug Free Years" curriculum on growth in alcohol use and risk for alcohol use in early adolescence. Prevention Science 2000;1:125-38.

* Spoth R, Lopez Reyes M, Redmond C, Shin C. Assessing a public health approach to delay onset and progression of adolescent substance use: latent transition and loglinear analyses of longitudinal family preventive intervention outcomes. Journal of Consulting and Clinical Psychology 1999a;67:619-30.

Spoth R, Redmond C, Lepper H. Alcohol initiation outcomes of universal family-focused preventive interventions: one- and two-year follow-ups of a controlled study. Journal of Studies on Alcohol 1999;Suppl 13:103-11.

Spoth R, Redmond C, Shin C, Azevedo K. Brief family intervention effects on adolescent substance initiation: schoollevel growth curve analyses 6 years following baseline. Journal of Consulting and Clinical Psychology 2004;72(3):535-42.

Spoth R, Shin C, Guyll M, Redmond C, Azevedo K. Universality of effects: an examination of the comparability of long-term family intervention effects on substance use across risk-related subgroups. Prevention Science 2006;7(2):209-24.

Spoth R, Trudeau L, Guyll M, Shin C, Redmond C. Universal intervention effects on substance use among young adults mediated by delayed adolescent substance initiation. Journal of Consulting and Clinical Psychology 2009;77(4):620-32.

Spoth RL, Redmond C, Shin C. Randomized trial of brief family interventions for general populations: adolescent substance use outcomes four years following baseline. Journal of Consulting and Clinical Psychology 2001;69(4):627-42.

Spoth 2002 \{published data only\}

Spoth R L, Redmond C, Trudeau L, Shin C. Longitudinal substance initiation outcomes for a universal preventive intervention combining family and school programs. Psychology of Addictive Behaviors 2002;16(2):129-34.

* Spoth R, Randall GK, Shin C, Redmond C. Randomized study of combined universal family and school preventive interventions: patterns of long-term effects on initiation, regular use, and weekly drunkenness. Psychology of Addictive Behaviors 2005;19(4):372-81.

Spoth R, Trudeau L, Redmond C, Shin C. Replicating and extending a model of effects of universal preventive intervention during early adolescence on young adult substance misuse. Journal of Consulting \& Clinical Psychology 2016;84(10):913-21.

Spoth RL, Randall GK, Trudeau L, Shin C, Redmond C. Substance use outcomes $51 / 2$ years past baseline for partnership-based, family-school preventive interventions. Drug and Alcohol Dependence 2008;96(1-2):57-68. 


\section{Stanger 2017 \{published data only\}}

Stanger C, Scherer EA, Babbin SF, Ryan SR, Budney AJ. Abstinence based incentives plus parent training for adolescent alcohol and other substance misuse. Psychology of Addictive Behaviors 2017;31(4):385-92.

\section{Stevens 2002 \{published data only\}}

Jones DJ, Olson AL, Forehand R, Gaffney CA, Zens MS, Bau JJ. A family-focused randomised controlled trial to prevent adolescent alcohol and tobacco use: the moderating roles of positive parenting and adolescent gender. Behavior Therapy 2005;36(4):347-55.

* Stevens MM, Olson AL, Gaffney CA, Tosteson TD, Mott LA, Starr P. A paediatric, practice-based, randomised trial of drinking and smoking prevention and bicycle helmet, gun, and seatbelt safety promotion. Pediatrics 2002;109(3):490-7.

\section{Stormshak 2011 \{published data only\}}

Stormshak EA, Connell AM, Veronneau MH, Myers MW, Dishion TJ, Kavanagh K, et al. An ecological approach to promoting early adolescent mental health and social adaptation: family-centered intervention in public middle schools. Child Development 2011;82(1):209-25.

* Van Ryzin MJ, Stormshak EA, Dishion TJ. Engaging parents in the family check-up in middle school: longitudinal effects on family conflict and problem behavior through the high school transition. Journal of Adolescent Health 2012;50(6):627-33.

Valdez 2013 \{published data only\}

Valdez A, Cepeda A, Parrish D, Horowitz R, Kaplan C. An adapted brief strategic family therapy for gang-affiliated Mexican American adolescents. Research on Social Work Practice 2013;23(4):383-96.

\section{Werch 2008 \{published data only\}}

* Werch CC, Moore MJ, Diclemente CC. Brief image-based health behavior messages for adolescents and their parents. Journal of Child and Adolescent Substance Abuse 2008;17(4):19-40.

\section{Winters 2012 \{published data only\}}

Piehler TF, Winters KC. Decision-making style and response to parental involvement in brief interventions for adolescent substance use. Journal of Family Psychology 2017;31(3):336-46.

Winters KC, Fahnhorst T, Botzet A, Lee S, Lalone B. Brief intervention for drug-abusing adolescents in a school setting: outcomes and mediating factors. Journal of Substance Abuse Treatment 2012;42(3):279-88.

* Winters KC, Lee S, Botzet A, Fahnhorst T, Nicholson A. Oneyear outcomes and mediators of a brief intervention for drug abusing adolescents. Psychology of Addictive Behaviors 2014;28(2):464-74.

\section{Wolchik 2002 \{published data only\}}

Wolchik SA, Sandler IN, Millsap RE, Plummer BA, Greene SM, Anderson ER, et al. Six-year follow-up of preventive interventions for children of divorce: a randomized controlled trial. JAMA 2002;288(15):1874-81.
* Wolchik SA, Sandler IN, Tein JY, Mahrer NE, Millsap RE, Winslow $E$, et al. Fifteen-year follow-up of a randomized trial of a preventive intervention for divorced families: effects on mental health and substance use outcomes in young adulthood. Journal of Consulting and Clinical Psychology 2013;81(4):660-73.

\section{Wu 2003 \{published data only\}}

Wu Y, Stanton BF, Galbraith J, Kaljee L, Cottrell L, Li X, et al. Sustaining and broadening intervention impact: a longitudinal randomized trial of 3 adolescent risk reduction approaches. Pediatrics 2003;111(1):e32-8.

\section{Wurdak 2017 \{published data only\}}

Wurdak M, Kuntsche E, Wolstein J. Effectiveness of an emailbased intervention helping parents to enhance alcoholrelated parenting skills and reduce their children's alcohol consumption - a randomised controlled trial. Drugs: Education, Prevention and Policy 2017;24(2):144-51.

\section{References to studies excluded from this review}

\section{Adolfsen 2017 \{published data only\}}

Adolfsen F, Strom HK, Martinussen M, Handegard BH, Natvig H, Eisemann $\mathrm{M}$, et al. Parent participation in alcohol prevention: evaluation of an alcohol prevention programme. Nordic Studies on Alcohol and Drugs 2017;34(6):456-70.

\section{Allen 2007 \{published data only\}}

Allen D, Coombes L, Foxcroft DR. Cultural accommodation of the Strengthening Families Programme 10-14: UK Phase I study. Health Education Research 2007;22(4):547-60.

Andreasson 2007 \{published data only\}

Andreasson S, Sjostrom E, Branstrom R. A six-community prevention trial to reduce alcohol and drug use-related problems in Sweden: planning and early findings. Substance Use \& Misuse 2007;42(12-13):2017-27.

Anonymous 2013 \{published data only\}

Anonymous. The impact of an alcohol education program using social norming. Kentucky Nurse 2013;61(1):9.

Barrett 2012 \{published data only\}

Barrett EL, Newton NC, Teesson M, Slade T, Conrod P. Modifying the personality-targeted 'preventure' program for use with Australian adolescents. Alcoholism: Clinical and Experimental Research 2012;36:350A.

\section{Bauman 2001 \{published data only\}}

Bauman KE, Ennett ST, Foshee VA, Pemberton M, Hicks K. Correlates of participation in a family-directed tobacco and alcohol prevention program for adolescents. Health Education \& Behavior 2001;28(4):440-61.

\section{Bauman 2001a \{published data only\}}

Bauman KE, Foshee VA, Ennett ST, Hicks K, Pemberton M. Family Matters: a family-directed program designed to prevent adolescent tobacco and alcohol use. Health Promotion Practice 2001;2(1):81-96 
Biglan 2000 \{published data only\}

Biglan A, Ary DV, Smolkowski K, Duncan T, Black C. A randomised controlled trial of a community intervention to prevent adolescent tobacco use. Tobacco Control 2000;9(1):24-32.

\section{Bobrowski 2014 \{published data only\}}

Bobrowski KJ, Pisarska A, Staszewski KO, Borucka A. [Effectiveness of alcohol prevention program for preadolescents]. Psychiatria Polska 2014;48(3):527-39.

\section{Brody 2004 \{published data only\}}

Brody GH, Murry VM, Gerrard M, Gibbons FX, Molgaard V, McNair L, et al. The Strong African American Families Program: translating research into prevention programming. Child Development 2004;75(3):900-17.

\section{Brody 2005 \{published data only\}}

Brody GH, McBride MV, McNair L, Chen Y, Gibbons FX, Gerrard M, et al. Linking changes in parenting to parent-child relationship quality and youth self-control: the Strong African American Families program. Journal of Research on Adolescence 2005;15(1):47-69.

\section{Brown 2014 \{published data only\}}

Brown PC, Dunn ME, Budney AJ. Development and initial evaluation of a web-based program to increase parental awareness and monitoring of underage alcohol use: a brief report. Journal of Child \& Adolescent Substance Abuse 2014;23(2):109-15.

\section{Byrnes 2010 \{published data only\}}

Byrnes HF, Miller BA, Aalborg AE, Plasencia AV, Keagy CD. Implementation fidelity in adolescent family-based prevention programs: relationship to family engagement. Health Education Research 2010;25(4):531-41.

\section{Calabria 2013 \{published data only\}}

Calabria B, Clifford A, Shakeshaft A, Allan J, Bliss D, Doran C. The acceptability to Aboriginal Australians of a family-based intervention to reduce alcohol-related harms. Drug and Alcohol Review 2013;32(3):328-32.

\section{Chilenski 2016 \{published data only\}}

Chilenski SM, Welsh JA, Perkins DF, Feinberg ME, Greenberg MT. Universal prevention exposure as a moderator of the community context: findings from the PROSPER project. American Journal of Community Psychology 2016;57(1-2):8-19.

\section{Cohen 1995 \{published data only\}}

Cohen DA, Linton KL. Parent participation in an adolescent drug abuse prevention program. Journal of Drug Education 1995;25(2):159-69.

\section{Connell 2007 \{published data only\}}

Connell AM, Dishion TJ, Yasui M, Kavanagh K. An adaptive approach to family intervention: linking engagement in family-centered intervention to reductions in adolescent problem behavior. Journal of Consulting \& Clinical Psychology 2007;75(4):568-79.

\section{Conrod 2009 \{published data only\}}

Conrod PJ, Castellanos N. Two-year drinking outcomes from the Preventure trial: a randomised controlled trial of personalitytargeted interventions. Alcoholism: Clinical and Experimental Research 2009;33:41A.

\section{Conrod 2011 \{published data only\}}

Conrod PJ, O'Leary-Barrett M, Newton N, Topper L, Castellanos$\mathrm{RN}$, MacKie C. Indirect, population-level effects of a selective youth alcohol prevention programme. Alcoholism: Clinical and Experimental Research 2011;35:316A.

DeGarmo 2009 \{published data only\} DeGarmo DS, Eddy JM, Reid JB, Fetrow RA. Evaluating mediators of the impact of the Linking the Interests of Families and Teachers (LIFT) multimodal preventive intervention on substance use initiation and growth across adolescence. Prevention Science 2009;10(3):208-20.

de Leeuw 2014 \{published data only\} de Leeuw RN, Kleinjan M, Lammers J, Lokman S, Engels RC. The effectiveness of the 'Healthy School and Drugs' project for primary schools. Kind en Adolescent 2014;35(1):2-21.

Donovan 2012 \{published data only\}

Donovan E, Wood M, Frayjo K, Black RA, Surette DA. A randomized, controlled trial to test the efficacy of an online, parent-based intervention for reducing the risks associated with college-student alcohol use. Addictive Behaviors 2012;37(1):25-35

\section{Ennett 2001 \{published data only\}}

Ennett ST, Bauman KE, Pemberton M, Foshee VA, Chuang YC, King TS, et al. Mediation in a family-directed program for prevention of adolescent tobacco and alcohol use. Preventive Medicine 2001;33(4):333-46.

\section{Epstein 2008 \{published data only\}}

Epstein JA, Griffin KW, Botvin GJ. A social influence model of alcohol use for inner-city adolescents: family drinking, perceived drinking norms, and perceived social benefits of drinking. Journal of Studies on Alcohol \& Drugs 2008;69(3):397-405.

\section{Faggiano 2007 \{published data only\}}

Faggiano F, Richardson C, Bohrn K, Galanti MR, Group EU-Dap Study. A cluster randomized controlled trial of school-based prevention of tobacco, alcohol and drug use: the EU-Dap design and study population. Preventive Medicine 2007;44(2):170-3.

\section{Fernandez 2011 \{published data only\}}

Fernandez AC, Wood MD, Laforge R, Black JT. Randomized trials of alcohol-use interventions with college students and their parents: lessons from the Transitions Project. Clinical Trials 2011;8(2):205-13.

\section{French 2008 \{published data only\}}

French MT, Zavala SK, McCollister KE, Waldron HB, Turner CW, Ozechowski TJ. Cost-effectiveness analysis of four interventions for adolescents with a substance use disorder. Journal of Substance Abuse Treatment 2008;34(3):272-81. 


\section{Gerrard 2006 \{published data only\}}

Gerrard M, Gibbons FX, Brody GH, Murry VM, Cleveland MJ, Wills TA. A theory-based dual-focus alcohol intervention for preadolescents: the Strong African American Families Program. Psychology of Addictive Behaviors 2006;20(2):185-95.

Gordon 2008 \{published data only\}

Gordon J, Biglan A, Smolkowski K. The impact on tobacco use of branded youth anti-tobacco activities and family communications about tobacco. Prevention Science 2008;9(2):73-87.

\section{Grossbard 2010 \{published data only\}}

Grossbard JR, Mastroleo NR, Kilmer JR, Lee CM, Turrisi R, Larimer ME, et al. Substance use patterns among firstyear college students: secondary effects of a combined alcohol intervention. Journal of Substance Abuse Treatment 2010;39(4):384-90.

\section{Haggerty 2006 \{published data only\}}

Haggerty KP, MacKenzie EP, Skinner ML, Harachi TW, Catalano RF. Participation in "Parents Who Care": predicting program initiation and exposure in two different program formats. Journal of Primary Prevention 2006;27(1):47-65.

\section{Hallgren 2011 \{published data only\}}

Hallgren MA, Sjolund T, Kallmen H, Andreasson S. Modifying alcohol consumption among high school students: an efficacy trial of an alcohol risk reduction program (PRIME for Life). Health Education 2011;111(3):216-29.

\section{Hawkins 2012 \{published data only\}}

Hawkins JD, Oesterle S, Brown EC, Monahan KC, Abbott RD, Arthur MW, et al. Sustained decreases in risk exposure and youth problem behaviors after installation of the Communities That Care prevention system in a randomized trial. Archives of Pediatrics \& Adolescent Medicine 2012;166(2):141-8.

\section{Hogue 2006 \{published data only\}}

Hogue A, Dauber S, Samuolis J, Liddle HA. Treatment techniques and outcomes in multidimensional family therapy for adolescent behavior problems. Journal of Family Psychology 2006;20(4):535-43.

\section{Ingels 2013 \{published data only\}}

Ingels JB, Corso PS, Kogan SM, Brody GH. Cost-effectiveness of the Strong African American Families-teen program: 1-year follow-up. Drug \& Alcohol Dependence 2013;133(2):556-61.

\section{Jackson 2016 \{published data only\}}

Jackson C, Ennett ST, Reyes HL, Hayes KA, Dickinson DM, Choi S, et al. Reducing children's susceptibility to alcohol use: effects of a home-based parenting program. Prevention Science 2016;17(5):615-25

\section{Jones 2007 \{published data only\}} Jones DJ, Foster SE, Olson AL, Forehand RL, Gaffney CA Zens MS, et al. Longitudinal retention of families in the assessment of a prevention program targeting adolescent alcohol and tobacco use: the utility of an ecological systems framework. Behavior Modification 2007;31(5):638-58.

\section{Komro 2006 \{published data only\}}

Komro KA, Perry CL, Veblen-Mortenson S, Farbakhsh K, Kugler KC, Alfano KA, et al. Cross-cultural adaptation and evaluation of a home-based program for alcohol use prevention among urban youth: the "Slick Tracy Home Team Program". Journal of Primary Prevention 2006;27(2):135-54.

\section{Komro 2017 \{published data only\}}

Komro KA, Livingston MD, Wagenaar AC, Kominsky TK Pettigrew DW, Garrett BA, et al. Multilevel prevention trial of alcohol use among American Indian and white high school students in the Cherokee nation. American Journal of Public Health 2017;107(3):453-9.

\section{Koning 2011 \{published data only\}}

Koning IM, van den Eijnden RJ, Engels RC, Verdurmen JE, Vollebergh WA. Why target early adolescents and parents in alcohol prevention? The mediating effects of self-control, rules and attitudes about alcohol use. Addiction 2011;106(3):538-46.

Koning 2012 \{published data only\}

Koning IM, Verdurmen JE, Engels RC, van den Eijnden RJ, Vollebergh WA. Differential impact of a Dutch alcohol prevention program targeting adolescents and parents separately and simultaneously: low self-control and lenient parenting at baseline predict effectiveness. Prevention Science 2012;13(3):278-87.

\section{Koning 2014 \{published data only\}}

Koning IM, van den Eijnden RJJM, Vollebergh WAM. Alcoholspecific parenting, adolescents' self-control, and alcohol use: a moderated mediation model. Journal of Studies on Alcohol and Drugs 2014;75(1):16-23.

\section{Koning 2015 \{published data only\}}

Koning IM, Maric M, MacKinnon D, Vollebergh WA. Effects of a combined parent-student alcohol prevention program on intermediate factors and adolescents' drinking behavior: a sequential mediation model. Journal of Consulting \& Clinical Psychology 2015;83(4):719-27.

Koning 2016 \{published data only\} Koning IM, Vollebergh WAM. Secondary effects of an alcohol prevention program targeting students and/or parents. Journal of Substance Abuse Treatment 2016;67:55-60.

\section{Kosterman 2001 \{published data only\}}

Kosterman R, Hawkins JD, Haggerty KP, Spoth R, Redmond C. Preparing for the drug free years: session-specific effects of a universal parent-training intervention with rural families. Journal of Drug Education 2001;31(1):47-68.

\section{Koutakis 2008 \{published data only\}}

Koutakis N, Stattin H, Kerr M. Reducing youth alcohol drinking through a parent-targeted intervention: the Orebro prevention program. Addiction 2008;103(10):1629-37.

\section{Liddle 2001 \{published data only\}}

Liddle HA, Dakof GA, Parker K, Diamond GS, Barrett K, Tejeda M. Multidimiensional family therapy for adolescent drug abuse: 
results of a randomized clinical trial. American Journal of Drug and Alcohol Abuse 2001;27(4):651-88.

\section{Litrownik 2000 \{published data only\}}

Litrownik AJ, Elder JP, Campbell NR, Ayala GX, Slymen DJ, Parra-Medina D, et al. Evaluation of a tobacco and alcohol use prevention program for Hispanic migrant adolescents: promoting the protective factor of parent-child communication. Preventive Medicine 2000;31(2 Pt 1):124-33.

Lowman 2004 \{published data only\}

Lowman C. Developing effective evidence-based interventions for adolescents with alcohol use disorders. Addiction 2004;99(Suppl 2):1-4.

\section{Malmberg 2014 \{published data only\}}

Malmberg M, Kleinjan M, Overbeek G, Vermulst A, Monshouwer K, Lammers J, et al. Effectiveness of the 'Healthy School and Drugs' prevention programme on adolescents' substance use: a randomized clustered trial. Addiction 2014;109(6):1031-40.

\section{Mares 2011 \{published data only\}}

Mares SH, van der Vorst H, Lichtwarck-Aschoff A, Schulten I, Verdurmen JE, Otten R, et al. Effectiveness of the home-based alcohol prevention program "In control: no alcohol!": study protocol of a randomized controlled trial. BMC Public Health 2011;11:622.

\section{McGillicuddy 2015 \{published data only\}}

McGillicuddy NB, Rychtarik RG, Papandonatos GD. Skill training versus 12-step facilitation for parents of substance-abusing teens. Journal of Substance Abuse Treatment 2015;50:11-7.

\section{Newton 2012 \{published data only\}}

Newton NC, Teesson M, Conrod P, Slade T, Barrett EL. Developing the cap intervention: a comprehensive model for alcohol and drug prevention. Alcoholism: Clinical and Experimental Research 2012;36:350A.

\section{Pentz 1989 \{published data only\}}

Pentz MA, Dwyer JH, MacKinnon DP, Flay BR, Hansen WB, Wang EY, et al. A multicommunity trial for primary prevention of adolescent drug abuse. Effects on drug use prevalence. JAMA 1989;261(22):3259-66

\section{Perry 1993 \{published data only\}}

Perry CL, Williams CL, Forster JL, Wolfson M, Wagenaar AC, Finnegan JR, et al. Background, conceptualization and design of a community-wide research program on adolescent alcohol use: Project Northland. Health Education Research 1993;8(1):125-36.

\section{Perry 1996 \{published data only\}}

Perry CL, Williams CL, Veblen-Mortenson S, Toomey TL, Komro KA, Anstine PS, et al. Project Northland: outcomes of a communitywide alcohol use prevention program during early adolescence. American Journal of Public Health 1996;86(7):956-65.

\section{Perry 2000 \{published data only\}}

Perry CL, Williams CL, Komro KA, Veblen-Mortenson S, Forster JL, Bernstein-Lachter R, et al. Project Northland high school interventions: community action to reduce adolescent alcohol use. Health Education \& Behavior 2000;27(1):29-49.

Perry 2002 \{published data only\}

Perry CL, Williams CL, Komro KA, Veblen-Mortenson S, Stigler $\mathrm{MH}$, Munson KA, et al. Project Northland: long-term outcomes of community action to reduce adolescent alcohol use. Health Education Research 2002;17(1):117-32.

Romero 2017 \{published data only\}

Romero E, Rodriguez C, Villar P, Gomez-Fraguela A. Intervention on early-onset conduct problems as indicated prevention for substance use: a seven-year follow up [Intervención sobre problemas de conductatempranos como prevención indicada del consumode drogas: siete años de seguimiento]. Adicciones 2017;29(3):150-62.

Rowe 2008 \{published data only\}

Rowe $\mathrm{CL}$, Liddle HA. Multidimensional family therapy for adolescent alcohol abusers. Alcoholism Treatment Quarterly 2008;26(1-2):105-23.

\section{Shortt 2007 \{published data only\}}

Shortt AL, Hutchinson DM, Chapman R, Toumbourou JW. Family, school, peer and individual influences on early adolescent alcohol use: first-year impact of the Resilient Families programme. Drug \& Alcohol Review 2007;26(6):625-34.

\section{Simons-Morton 2005 \{published data only\}}

Simons-Morton B, Haynie D, Saylor K, Crump AD, Chen R. The effects of the Going Places program on early adolescent substance use and antisocial behavior. Prevention Science 2005;6(3):187-97.

\section{Simons-Morton 2005a \{published data only\}}

Simons-Morton B, Haynie D, Saylor K, Crump AD, Chen R. Impact analysis and mediation of outcomes: the Going Places program. Health Education \& Behavior 2005;32(2):227-41.

\section{Spoth 2002a \{published data only\}}

Spoth RL, Guyll M, Day SX. Universal family-focused interventions in alcohol-use disorder prevention: costeffectiveness and cost-benefit analyses of two interventions. Journal of Studies on Alcohol 2002;63(2):219-28.

\section{Spoth 2007 \{published data only\}}

Spoth R, Redmond C, Shin C, Greenberg M, Clair S, Feinberg M. Substance-use outcomes at 18 months past baseline: the PROSPER Community-University Partnership Trial [Erratum appears in American Journal of Preventive Medicine 2015 Jan;48(1):120]. American Journal of Preventive Medicine 2007;32(5):395-402.

\section{Spoth 2013 \{published data only\}}

Spoth R, Redmond C, Shin C, Greenberg M, Feinberg M, Schainker L. PROSPER community-university partnership delivery system effects on substance misuse through $61 / 2$ years 
past baseline from a cluster randomized controlled intervention trial. Preventive Medicine 2013;56(3-4):190-6.

\section{Spoth 2014 \{published data only\}}

Spoth R, Trudeau L, Redmond C, Shin C. Replication RCT of early universal prevention effects on young adult substance misuse. Journal of Consulting \& Clinical Psychology 2014;82(6):949-63.

\section{Stanton 2004 \{published data only\}}

Stanton B, Cole M, Galbraith J, Li X, Pendleton S, Cottrel L, et al. Randomized trial of a parent intervention: parents can make a difference in long-term adolescent risk behaviors, perceptions, and knowledge. Archives of Pediatrics \& Adolescent Medicine 2004;158(10):947-55.

\section{Stigler 2006 \{published data only\}}

Stigler MH, Perry CL, Komro KA, Cudeck R, Williams CL. Teasing apart a multiple component approach to adolescent alcohol prevention: what worked in Project Northland?. Prevention Science 2006;7(3):269-80.

\section{Teesson 2013 \{published data only\}}

Teesson M, Newton N, Slade T, Conrod P. An integrated approach to substance use prevention for high-and lowrisk youth: the CAP intervention. Alcoholism: Clinical and Experimental Research 2013;37:148A.

\section{Tomczyk 2015 \{published data only\}}

Tomczyk S, Hanewinkel R, Isensee B. 'Klar bleiben': a schoolbased alcohol prevention programme for German adolescents - study protocol for a cluster randomised controlled trial. BMJ Open 2015;5(11):e010141.

\section{Turrisi 2013 \{published data only\}}

Turrisi R, Mallett KA, Cleveland MJ, Varvil-Weld L, Abar C, Scaglione N, et al. Evaluation of timing and dosage of a parent-based intervention to minimize college students' alcohol consumption. Journal of Studies on Alcohol \& Drugs 2013;74(1):30-40.

\section{University of Minnesota 2015 \{published data only\}}

University of Minnesota Clinical, Translational Science Institute, National Cancer Institute. Family-skills training to prevent tobacco and other substance use in Latino youth. ClinicalTrials.gov NCT014427532015.

\section{University of Minnesota 2018 \{published data only\}}

University of Minnesota, Clinical and Translational Science Institute. Latino fathers promoting healthy youth behaviors. ClinicalTrials.gov NCT01442753 2018.

\section{University of Rhode Island 2011 \{published data only\}}

University of Rhode Island. Tailored interventions to prevent substance abuse. ClinicalTrials.gov NCT01442753 2011.

\section{Valentine 1998 \{published data only\}}

Valentine J, Gottlieb B, Keel S, Griffith J, Ruthazer R. Measuring the effectiveness of the Urban Youth Connection: the case for dose-response modeling to demonstrate the impact of an adolescent substance abuse prevention program. Journal of Primary Prevention 1998; Vol. 18, issue 3:363-87.

\section{Verdurmen 2014 \{published data only\}}

Verdurmen JE, Koning IM, Vollebergh WA, van den Eijnden RJ, Engels RC. Risk moderation of a parent and student preventive alcohol intervention by adolescent and family factors: a cluster randomized trial. Preventive Medicine 2014;60:88-94.

\section{Vermeulen-Smit 2014 \{published data only\}}

Vermeulen-Smit E, Mares SHW, Verdurmen JEE, Van der Vorst $H$, Schulten IGH, Engels RCME, et al. Mediation and moderation effects of an in-home family intervention: the "In control: No alcohol!” pilot study. Prevention Science 2014;15(5):633-42.

\section{Veronneau 2016 \{published data only\}}

Veronneau M-H, Dishion TJ, Connell AM, Kavanagh K. A randomized, controlled trial of the family check-up model in public secondary schools: examining links between parent engagement and substance use progressions from early adolescence to adulthood. Journal of Consulting and Clinical Psychology 2016;84(6):526-43.

\section{Watson 2017 \{published data only\}}

Watson J, Toner P, Day E, Back D, Brady LM, Fairhurst C, et al. Youth Social Behaviour and Network Therapy (Y-SBNT): adaptation of a family and social network intervention for young people who misuse alcohol and drugs - a randomised controlled feasibility trial. Health Technology Assessment 2017;21(15):1-259.

\section{Werch 1998 \{published data only\}}

Werch CE, Pappas DM, Carlson JM, DiClemente CC. Shortand long-term effects of a pilot prevention program to reduce alcohol consumption. Substance Use \& Misuse 1998;33(11):2303-21.

\section{Werch 1999 \{published data only\}}

Werch CE, Pappas DM, Carlson JM, DiClemente CC. Six-month outcomes of an alcohol prevention program for inner-city youth. American Journal of Health Promotion 1999;13(4):237-40, iii.

\section{Werch 2003 \{published data only\}}

Werch CE, Owen DM, Carlson JM, DiClemente CC, Edgemon P, Moore M. One-year follow-up results of the STARS for Families alcohol prevention program. Health Education Research 2003;18(1):74-87.

\section{Werch 2010 \{published data only\}}

Werch CE, Bian H, Diclemente CC, Moore MJ, Thombs D, Ames SC, et al. A brief image-based prevention intervention for adolescents. Psychology of Addictive Behaviors 2010;24(1):170-5.

\section{Williams 1995 \{published data only\}}

Williams CL, Perry CL, Dudovitz B, Veblen-Mortenson S, Anstine PS, Komro KA, et al. A home-based prevention program for sixth-grade alcohol use: results from Project Northland. Journal of Primary Prevention 1995;16(2):125-47.

Williams 2001 \{published data only\}

Williams CL, Grechanaia T, Romanova O, Komro KA, Perry CL, Farbakhsh K. Russian-American Partners for Prevention. Adaptation of a school-based parent-child programme for 
alcohol use prevention. European Journal of Public Health 2001;11(3):314-21.

\section{Wolchik 2003 \{published data only\}}

Wolchik SA, Millsap RE. Group based interventions for mothers and mothers plus children reduced mental health problems in adolescent children of divorced parents. Evidence Based Nursing 2003; Vol. 6:74.

\section{Zanetta 2008 \{published data only\}}

Zanetta R, Moacyr R, Lancarotte I. Bringing up students in the Healthy Lifestyle Multiplier Students program, Sao Paulo, Brazil. Preventing Chronic Disease 2008;5(3):A98.

\section{References to studies awaiting assessment}

\section{Danielson 2016 \{published data only\}}

Danielson CK. Reducing risk for substance use problems among adolescents with a child maltreatment history. Journal of the American Academy of Child and Adolescent Psychiatry 2016;55(10 Suppl 1):S293.

\section{Ford 2018 \{published data only\}}

Ford CA, Mirman JH, Garcia-Espana JF, Friedrich E, Thiel MCF. Influence of primary care-based parent-targeted intervention on parent-teen communication about sexual and alcohol behaviors: a randomized controlled trial. Journal of Adolescent Health. Society for Adolescent Health and Medicine Annual Meeting 2018. United States, 2018; Vol. 62 (Suppl 1):S33-S34.

\section{Noel 2015 \{published data only\}}

Noel V, Stanger C, Budney AJ. Parent tobacco use, monitoring, and impulsive decision making: predictors of post treatment youth substance use. Drug and Alcohol Dependence 2015;146:e53.

\section{Winters 2015 \{published data only\}}

Winters K. Parents as interventionists to address adolescent drug abuse. Drug and Alcohol Dependence 2015;156:e240.

\section{References to ongoing studies}

\section{Allen 2012 \{published data only\}}

Allen ML, Garcia-Huidobro D, Hurtado GA, Allen R, Davey CS, Forster JL, et al. Immigrant family skills-building to prevent tobacco use in Latino youth: study protocol for a communitybased participatory randomized controlled trial. Trials 2012;13:242.

\section{Bukstein 2006 \{published data only\}}

Bukstein O. Home-based Behavioral Therapy (HBT): Psychosocial Intervention Project for Early Adolescents With Pre- or Early Substance Use Disorder: Phase II. ClinicalTrials.gov 2006.

\section{Conrod 2017 \{published data only\}}

Conrod P. Inter-Venture: A Cluster Randomized Controlled Trial Investigating the Effect of School-based Personality-targeted Interventions and Collaborative Youth Mental Health Care. ClinicalTrials.gov 2017.
Ford 2015 \{published data only\}

Ford C. A Pilot Efficacy and Implementation Study of the Patients, Parents and Professionals Partnering to Improve Adolescent Health (P4) Intervention. ClinicalTrials.gov 2015.

Hops 2012 \{published data only\}

Hops H. Early Intervention for Minors in Possession of Alcohol/ Drugs: A Feasibility Study. ClinicalTrials.gov 2016.

\section{Kogan 2018 \{published data only\}}

Kogan S. Strong African American Families STEPS Project (SAAFSTEPS). ClinicalTrials.gov July 2018.

McCart 2017 \{published data only\}

McCart M. Experimental Mediation Research Aimed at Enhancing Adolescent Substance Abuse Treatment. ClinicalTrials.gov 2017, updated June 2018.

Mello 2016 \{published data only\} Mello M. An e-Parenting Skills Intervention to Decrease Injured Adolescents' Alcohol Use. ClinicalTrials.gov 2016, updated April 2018.

Miller 2009 \{published data only\}

Miller B. Adolescent Family-Based Alcohol Prevention. ClinicalTrials.gov 2009.

Miller 2018 \{published data only\} Miller B. Family Based Prevention of Alcohol and Risky Sex for Older Teens. ClinicalTrials.gov May 2018.

Rowe 2010 \{published data only\}

Rowe C. Family Intervention for Teen Drinking and AlcoholRelated Crises in the ER. ClinicalTrials.gov 2010.

\section{Segrott 2014 \{published data only\}}

Segrott J, Gillespie D, Holliday J, Humphreys I, Murphy S, Phillips C, et al. Preventing substance misuse: study protocol for a randomised controlled trial of the Strengthening Families Programme 10-14 UK (SFP 10-14 UK). BMC Public Health 2014;14:49. [DOI: 10.1186/1471-2458-14-49; Current Controlled Trials ISRCTN63550893]

\section{Sheidow 2017 \{published data only\}}

Sheidow A. Improving Access to Substance Abuse EvidenceBased Practices for Youth in the Justice System: Strategies Used by JPOs. ClinicalTrials.gov 2017, updated April 2018.

Spirito 2017b \{published data only\}

Spirito A. Computer-Assisted Brief Intervention Protocol for Marijuana Using Juvenile Offenders. ClinicalTrials.gov 2017, updated January 2018.

Stanton 2007 \{published data only\}

Stanton M. Family and Group Therapies for Adolescent Alcohol Abuse. ClinicalTrials.gov 2007.

Whitbeck 2016 \{published data only\}

Whitbeck L. A Randomized Control Trial (RCT) of a FamilyCentered Ojibwe Substance Abuse Prevention. ClinicalTrials.gov 2016. 


\section{Additional references}

\section{Aiken 2018}

Aiken A, Clare P, Wadolowski M, Hutchinson D, Najman J, Slade T, et al. Age of alcohol initiation and progression to binge drinking in adolescence: a prospective cohort study. Alcoholism: Clinical and Experimental Research 2018;42(1):100-10.

\section{Alati 2014}

Alati R, Baker P, Betts K, Connor J, Little K, Sanson A, et al. The role of parental alcohol use, parental discipline and antisocial behaviour on adolescent drinking trajectories. Drug and Alcohol Dependence 2014;134:178-184.

\section{Bava 2010}

Bava S, Tapert SF. Adolescent brain development and the risk for alcohol and other drug problems. Neuropsychological Reviews 2010;20:398-413.

\section{Bonomo 2004}

Bonomo YA, Bowes G, Coffey C, Carlin JB, Patton GC. Teenage drinking and the onset of alcohol dependence: a cohort study over seven years. Addiction 2004;99:1520-8.

\section{Caria 2011}

Caria MP, Faggiano F, Bellocco R, Galanti R. The influence of socioeconomic environment on the effectiveness of alcohol prevention among European students: a cluster randomised controlled trial. BMC Public Health 2011;11(312):8. [DOI: 10.1186/1471-2458-11-312]

\section{Carter 2007}

Carter M, McGee R, Taylor B, Williams S. Health outcomes in adolescence: associations with family, friends and school engagement. Journal of Adolescence 2007;30:51-62.

\section{Chassin 1996}

Chassin L, Curran PJ, Hussong AM, Colder CR. The relation of parent alcoholism to adolescent substance use: a longitudinal follow-up study. Journal of Abnormal Psychology 1996;105:70-80.

\section{Courtney 2009}

Courtney KE, Polich J. Binge drinking in young adults: data, definitions, and determinants. Psychological Bulletin 2009;135:142-56.

\section{Cranford 2010}

Cranford JA, Zucker RA, Jester JM, Puttler LI, Fitzgerald HE. Parental alcohol involvement and adolescent alcohol expectancies predict alcohol involvement in male adolescents. Psychology of Addictive Behaviours 2010;24(3):386-96.

\section{Currie 2012}

Currie C, Zanotti C, Morgan A, Currie D, de Looze M, Roberts C, et al. Social determinants of health and well-being among young people. Health Behaviour in School-aged Children (HBSC) study: international report from the 2009/2010 survey, Health Policy for Children and Adolescents, No. 6. Copenhagen: WHO Regional Office for Europe, 2012.

\section{DeWit 2000}

DeWit DJ, Adlaf EM, Offord DR, Ogborne AC. Age at first alcohol use: a risk factor for the development of alcohol disorders. American Journal of Psychiatry 2000;157:745-50.

\section{Dusendury 2000}

Dusenbury L. Family-based drug abuse prevention programs: a review. Journal of Primary Prevention 2000;20(4):337-52.

\section{EMCDDA 2015}

EMCDDA. Prevention of Drug Use. Lisbon, Portugal: European Monitoring Centre for Drugs and Drug Addiction,. European Monitoring Centre for Drugs and Drug Addiction, 2015.

\section{Engels 2007}

Engels RC, De Leeuw RNH, Poelen EP. The impact of parents on adolescent drinking and friendship selection process. In: Jarvinen M, Room R editor(s). Youth Drinking Cultures: European Experiences. Hampshire, England: Ashgate Publishing, 2007.

\section{Foxcroft 2011b}

Foxcroft DR, Tsertsvadze A. Universal school-based prevention programs for alcohol misuse in young people. Cochrane Database of Systematic Reviews 2011, Issue 5. [DOI: 10.1002/14651858.CD009113]

\section{Foxcroft 2011c}

Foxcroft DR, Tsertsvadze A. Universal multi-component prevention programs for alcohol misuse in young people. Cochrane Database of Systematic Reviews 2011, Issue 9. [DOI: 10.1002/14651858.CD009307]

\section{Foxcroft 2014}

Foxcroft DR. Can prevention classification be improved by considering the function of prevention?. Prevention Science 2014;15(6):818-22.

\section{Frieden 2014}

Frieden T, Jaffe HW, Cono J, Richards C, Iademarco MF. Youth Risk Behaviour Surveillance - United States 2013. Surveillance Summaries. Atlanta, GA: Centers for Disease Control and Prevention, US Department of Human Services, 2014; Vol. 63:1-168.

\section{Gates 2006}

Gates S, McCambridge J, Smith LA, Foxcroft D. Interventions for prevention of drug use by young people delivered in non-school settings. Cochrane Database of Systematic Reviews 2006, Issue 1. [DOI: 10.1002/14651858.CD005030.pub2]

\section{GRADE 2004}

The GRADE Working Group. Grading quality of evidence and strength of recommendations. BMJ 2004;328(7454):1490.

\section{Guyatt 2008}

Guyatt GH, Oxman AD, Vist GE, Kunz R, Falck-Ytter Y, AlonsoCoello $P$, et al. GRADE: an emerging consensus on rating quality of evidence and strength of recommendations. $B M J$ 2008;336(7560):924-6. 


\section{Guyatt 2011}

Guyatt G, Oxman AD, Akl EA, Kunz R, Vist G, Brozek J, et al. GRADE guidelines 1. Introduction - GRADE evidence profiles and summary of findings tables. Journal of Clinical Epidemiology 2011;64:383-94.

\section{Hale 2014}

Hale DR, Fitzgerald-Yau N, Viner RM. A systematic review of effective interventions for reducing multiple health risk behaviors in adolescence. American Journal of Public Health 2014;104(5):e19-41.

\section{Hanson 2007}

Hanson MD, Chen E. Socioeconomic status and health behaviours in adolescence: a review of the literature. Journal of Behavioural Medicine 2007;30:263-85.

\section{Heginbotham 2012}

Heginbotham C. Values-Based Commissioning of Health and Social Care. Cambridge, UK: Cambridge University Press, 2012.

\section{Hibell 2012}

Hibell B, Ulf Guttormsson U, Ahlström S, Balakireva O, Bjarnason T, Kokkevi A, et al. The 2011 ESPAD Report: substance use among students in 36 European countries. http://www.espad.org/en/references--literature/the-2011espad-report---substa/. Stockholm: The Swedish Council for Information on Alcohol and other Drugs (CAN), The European Monitoring Centre for Drugs and Drug Addiction (EMCDDA), Council of Europe, Co-operation Group to Combat Drug Abuse and Illicit Trafficking in Drugs (Pompidou Group), 2012.

\section{Higgins 2011}

Higgins JPT, Green S (editors). Cochrane Handbook for Systematic Reviews of Interventions Version 5.1.0. The Cochrane Collaboration, 2011. Available from www.cochranehandbook.org.

\section{Hingson 2014}

Hingson R, White A. New research findings since the 2007 Surgeon General's Call to Action to Prevent and Reduce Underage Drinking: a review. Journal of Studies on Alcohol and Drugs 2014;75(1):158-69.

\section{Jackson 2015}

Jackson KM, Barnett NP, Colby SM, Rogers ML. The prospective association between sipping alcohol by the sixth grade and later substance use. Journal of Studies on Alcohol and Drugs 2015;76:212-21.

\section{Jimenez-Iglesias 2013}

Jimenez-Iglesias A, Moreno C, Rivera F, García-Moya I. The role of the family in promoting responsible substance use in adolescence. Journal of Child and Family Studies 2013;22:585-602.

\section{Kuntsche 2013}

Kuntsche E, Rossow I, Simons-Morton B, Bogt TT, Kokkevi A, Godeau E. Not early drinking but early drunkenness is a risk factor for problem behaviors among adolescents from 38
European and North American countries. Alcoholism: Clinical and Experimental Research 2013;37:308-14.

\section{Kuntsche 2016}

Kuntsche S, Kuntsche E. Parent-based interventions for preventing or reducing adolescent substance use - a systematic literature review. Clinical Psychology Review 2016;45:89-101.

\section{Lemstra 2010}

Lemstra M, Bennett N, Nannapaneni U, Neudorf C, Warren L, et al. A systematic review of school based marijuana and alcohol prevention programs targeting adolescents aged 10-15. Addiction Research and Theory 2010;18(1):84-96.

\section{Lim 2012}

Lim SS. A comparative risk assessment of burden of disease and injury attributable to 67 risk factors and risk factor clusters in 21 regions, 1990-2010: a systematic analysis for the Global Burden of Disease Study 2010. Lancet 2012;380(9859):2224-60.

\section{Livingston 2008}

Livingston M, Laslett AM, Dietze P. Individual and community correlates of young people's high-risk drinking in Victoria, Australia. Drug and Alcohol Dependence 2008;98(3):241-8.

\section{Lowry 1996}

Lowry R, Kann L, Collins JL, Kolbe LJ. The effect of socioeconomic status on chronic disease risk behaviours among US adolescents. JAMA 1996;276:792-7.

\section{MacArthur 2012}

MacArthur G, Kipping R, White J, Chittleborough C, Lingam R, Pasch K, et al. Individual-, family-, and school-level interventions for preventing multiple risk behaviours in individuals aged 8 to 25 years. Cochrane Database of Systematic Reviews 2012, Issue 6. [DOI: 10.1002/14651858.CD009927.pub2]

\section{Marmot 2010}

Marmot M. Fair Society, Healthy Lives. The Marmot Review. London, UK: Institute of Health Equity,. www.ucl.ac.uk/ marmotreview, 2010.

\section{McMorris 2011}

McMorris BJ, Catalano RF, Kim MJ, Toumbourou JW, Hemphill SA. Influence of family factors and supervised alcohol use on adolescent alcohol use and harms: similarities between youth in different alcohol policy contexts. Journal of Studies on Alcohol and Drugs 2011;72(3):418-28.

\section{Mrazek 1994}

Mrazek PJ, Haggerty RJ (editors). Reducing Risks for Mental Disorders: Frontiers for Preventive Intervention Research. Washington DC: Institute of Medicine, 1994.

\section{NHMRC 2009}

National Health and Medical Research Council. Australian Guidelines to Reduce Health Risks from Drinking Alcohol. Canberra: Commonwealth of Australia, 2009. 


\section{Patton 2004}

Patton GC, McMorris BJ, Toumbourou JW, Hemphill SA, Donath S, Catalano RF. Puberty and the onset of substance use and abuse. Pediatrics 2004;114:e300-6.

\section{Petrie 2007}

Petrie J, Bunn F, Byrne G. Parenting programmes for preventing tobacco, alcohol, or drugs misuse in children < 18: a systematic review. Health Education Research 2007;22(2):177-91.

\section{Quinn 2011}

Quinn PD, Fromme K. Predictors and outcomes of variability in subjective alcohol intoxication among college students: an event-level analysis across 4 years. Alcoholism: Clinical and Experimental Research 2011;35(3):484-95.

\section{Reboussin 2010}

Reboussin BA, Preisser JS, Song EY, Wolfson M. Geographic clustering of underage drinking and the influence of community characteristics. Drug and Alcohol Dependence 2010;106(1):38-47.

\section{Rehm 2010}

Rehm J, Baliunas D, Borges GL, Graham K, Irving H, Kehoe T, et al. The relation between different dimensions of alcohol consumption and burden of disease: an overview. Addiction 2010;105(5):817-43.

\section{Rehm 2012}

Rehm J, Shield, K, Rehm M, Gmel G, Frick U. Alcohol consumption, alcohol dependence and attributable burden of disease in Europe: potential gains from effective interventions for alcohol dependence. Centre for Addiction and Mental Health; www.camh.net. 2012.

\section{Rehm 2013}

Rehm J, Shield KD. Global alcohol-attributable deaths from cancer, liver cirrhosis, and injury in 2010. Alcohol Research: Current Reviews 2013;35(2):174-83.

\section{RevMan 2014 [Computer program]}

Copenhagen: The Nordic Cochrane Centre, The Cochrane Collaboration. Review Manager (RevMan). Version 5.3. Copenhagen: The Nordic Cochrane Centre, The Cochrane Collaboration, 2014

\section{Richter 2009}

Richter M, Vereecken CA, Boyce W, Maes L, Gabhainn SN, Currie CE. Parental occupation, family affluence and adolescent health behaviour in 28 countries. International Journal of Public Health 2009;54(4):203-12.

\section{Room 2004}

Room R. Drinking and coming of age in a cross-cultural perspective. In: Bonnie RJ, O'Connor ME editor(s). Reducing Underage Drinking: A Collective Responsibility. Washington, DC: National Academy Press, 2004

\section{Ryan 2010}

Ryan SM, Jorm AF, Lubman DI. Parenting factors associated with reduced adolescent alcohol use: a systematic review of longitudinal studies. Australian and New Zealand Journal of Psychiatry 2010;44(9):774-83.

\section{Simons-Morton 2009}

Simons-Morton BG, Farhat T, Ter Bogt TF, Hublet A, Kuntsche E, Nic Gabhainn S, et al. Gender specific trends in alcohol use: cross-cultural comparisons from 1998 to 2006 in 24 countries and regions. International Journal of Public Health 2009;54(Suppl 2):199-208.

\section{Smit 2008}

Smit E, Verdurmen J, Monshouwer K, Smit F. Family interventions and their effect on adolescent alcohol use in general populations: a meta-analysis of randomised controlled trials. Drug and Alcohol Dependence 2008;97:195-206.

\section{Springer 2006}

Springer F, Phillips JL. The IOM model: a tool for prevention planning and implementation. Prevention Tactics 2006;8(13):1-8.

\section{Steinberg 2001}

Steinberg L. We know some things: parent-adolescent relationships in retrospect and prospect. Journal of Research on Adolescence 2001;11(1):1-19.

\section{Turrisi 2010}

Turrisi R, Ray AE. Sustained parenting and college drinking in first-year students. Developmental Psychobiology 2010;52(3):286-94.

\section{USDHHS 2015}

U.S. Department of Health and Human Services and U.S. Department of Agriculture. 2015 - 2020 Dietary Guidelines for Americans, 8th Edition. Washington, DC: USDHHS, 2015.

\section{Vermeulen-Smit 2015}

Vermeulen-Smit E, Verdurmen JEE, Engels RCME. The effectiveness of family interventions in preventing adolescent illicit drug use: a systematic review and meta-analysis of randomised controlled trials. Clinical Child and Family Psychological Review 2015;18(3):218-39.

\section{Waller 2018}

Waller R, Murray L, Shaw D, Forbes E, Hyde L. Accelerated alcohol use across adolescence predicts early adult symptoms of alcohol use disorder via reward-related neural function. Psychological Medicine April 2018;doi.org/10.1017/ S003329171800137X:1-10.

\section{Wang 2009}

Wang J, Simons-Morton BG, Farhat T, Luk JW. Sociodemographic variability in adolescent substance use: mediation by parents and peers. Prevention Science 2009;10(4):387-96.

\section{White 2000}

White HR, Johnson V, Buyske S. Parental modeling and parenting behavior effects on offspring alcohol and cigarette use: a growth curve analysis. Journal of Substance Abuse 2000;12(3):287-310. 


\section{White 2010}

White J, Halliwell E. Alcohol and tobacco use during adolescence: the importance of the family mealtime environment. Journal of Health Psychology 2010;15(4):526-32.

\section{White 2012}

White V, Bariola E. Australian secondary school students' use of tobacco, alcohol, and over-the-counter and illicit substances in 2011: report. Canberra, Australia: National Drug Strategy, Department of Health and Ageing, 2012.

\section{WHO 2012}

WHO. Alcohol in the European Union. Consumption, Harm, and Policy Approaches. Copenhagen, Denmark: World Health Organization,. Copenhagen, Denmark: World Health Organisation, Regional Office for Europe, 2012.

\section{CHARACTERISTICS OF STUDIES}

Characteristics of included studies [ordered by study ID]

\section{Wills 2003}

Wills TA, Yaeger AM. Family factors and adolescent substance use: models and mechanisms. Current Directions in Psychological Science 2003;12:222-6.

\section{References to other published versions of this review Foxcroft 2011a}

Foxcroft DR, Tsertsvadze A. Universal family-based prevention programs for alcohol misuse in young people. Cochrane Database of Systematic Reviews 2011, Issue 9. [DOI: 10.1002/14651858.CD009308]

* Indicates the major publication for the study

\section{Arnaud 2016}

\begin{tabular}{ll}
\hline Methods & Design: cluster RCT \\
& Follow-up: $3 \& 6 \mathrm{mo}$ (post randomisation) \\
& Attrition: $15.9 \%(3 \mathrm{mo}), 12.8 \%(6 \mathrm{mo})$ \\
& Unit of randomisation: paediatric emergency department \\
\hline Participants & N of clusters (subjects) randomised: \\
Int: NR (144) \\
Ctrl: NR (176) \\
Total N: $6(320)$ \\
N of subjects 6 mo data: \\
Int: NR (126) \\
Ctrl: NR (153) \\
Total N: $6(279)$ \\
Age: mean: Int $15.7(\mathrm{SD}=1.2)$, Ctrl $15.8(\mathrm{SD}=1.2)$ \\
Sex (male): Int $48.9 \%$, Ctrl $52.0 \%$ \\
Ethnicity: $\mathrm{NR}$ \\
Country: Germany
\end{tabular}

Interventions Number of experimental conditions: 2

Description of Intervention: manual guided b-MI intervention for youth based on principles of motivational interviewing (MI), motivational enhancement interventions, and directive counselling. Uses principles of reflective listening; empathic feedback; non-confronting and non-judgemental assessment; exploration of drinking-related attitudes, norms, and goals; goal attainment strategies; reflective summary emphasising personal responsibility; a written agreement on behaviour goals; and provision of relevant contact information of youth-specific services. Parent(s) received a brief consultation including general information on youth alcohol use and encouragement to reflect on the episode and to develop strategies to prevent future risky alcohol use or seek further services. Parent(s), youth, and counsellors gathered to create a summary statement focusing on supporting the adolescent's self-efficacy regarding his or her alcohol-related goals. Participants contacted by telephone 6 weeks after to reinforce motivation for reaching goals

Type of intervention: indicated (adolescents recruited from paediatric emergency department with acute alcohol intoxication as primary diagnosis)

Focus/target: alcohol use and related problems Fidelity: manual guided, \% NR

Dose: $97.9 \%$ received b-MI, $51.7 \%$ received parental consultation, $74.5 \%$ received booster 
Arnaud 2016 (Continued)

Duration/frequency: 45 min youth session; parent and parent, youth, counsellor session duration NR; 5 to 10 minute telephone contact 6 weeks following initial intervention

Control: treatment as usual; received information leaflet on negative consequences of alcohol misuse and contact details of the youth substance use counselling service with recommendation for contact
Funding and Declared Conflicts of Interest
Length of follow-up: $3 \& 6$ mo (post randomisation)

\section{Alcohol outcomes:}

$\%$ of youth reporting binge drinking; mean binge drinking frequency

Number of drinks consumed on a typical drinking occasion

Alcohol-related problems assessed with the brief Rutgers Alcohol Problems Index

This research was supported by a grant from the German Federal Ministry for Education and Research (BMBF), 01KQ1002B. Study authors declared no conflicts of interest

Notes

\section{Abbreviations:}

Ctrl=control; Int=intervention; mo=month(s); N=number; NR=not reported; RCT=randomised controlled trial; $y r(s)=y e a r(s)$

\section{Risk of bias}

\begin{tabular}{|c|c|c|}
\hline Bias & Authors' judgement & Support for judgement \\
\hline $\begin{array}{l}\text { Random sequence genera- } \\
\text { tion (selection bias) }\end{array}$ & Unclear risk & Not described \\
\hline $\begin{array}{l}\text { Allocation concealment } \\
\text { (selection bias) }\end{array}$ & Unclear risk & Not described \\
\hline $\begin{array}{l}\text { Blinding of participants } \\
\text { and personnel (perfor- } \\
\text { mance bias) } \\
\text { All outcomes }\end{array}$ & High risk & Blinding not possible due to the nature of the intervention \\
\hline $\begin{array}{l}\text { Blinding of outcome as- } \\
\text { sessment (detection bias) } \\
\text { All outcomes }\end{array}$ & High risk & Self-reported \\
\hline $\begin{array}{l}\text { Incomplete outcome data } \\
\text { (attrition bias) } \\
\text { All outcomes }\end{array}$ & Low risk & Attrition low, no differential attrition by group, imputation performed \\
\hline $\begin{array}{l}\text { Selective reporting (re- } \\
\text { porting bias) }\end{array}$ & Unclear risk & $\begin{array}{l}\text { Reported outcomes consistent with the clinical trial registry: ISRCTN identifier: } \\
\text { ISRCTN31234060 }\end{array}$ \\
\hline Other bias & Unclear risk & $\begin{array}{l}\text { Contamination not described but is possible due to switch of clinics between } \\
\text { intervention and control in different weeks }\end{array}$ \\
\hline Recruitment to cluster & High risk & Recruitment of individuals to clusters after clusters randomised \\
\hline Baseline imbalances & Unclear risk & Unclear if baseline imbalances accounted for in analysis \\
\hline Loss of clusters & Low risk & No report of loss of entire clusters \\
\hline Incorrect analysis & Low risk & Analysis adjusted for clustering \\
\hline
\end{tabular}


Arnaud 2016 (Continued)
Compatability with indi-
Unclear risk
Unable to determine if herd effect exists

vidually randomised trials

(herd effect)

Baldus 2016

$\begin{array}{ll}\text { Methods } & \text { Design: } \mathrm{RCT} \\ & \text { Follow-up: } 8 \mathrm{wk}, 6 \& 18 \mathrm{mo} \text { (post randomisation) } \\ & \text { Attrition: } 9.9 \%(8 \mathrm{wk}), 13.0 \% \text { (6 mo), 15.3\% (18 mo) } \\ & \text { Unit of randomisation: parent-adolescent dyad }\end{array}$

Participants

N of subjects randomised:

Int: 153 families

Ctrl: 149 families

Total N: 302 families

N of subjects 18 mo data:

Int: 135 families (132 adolescents)

Ctrl: 127 families (125 adolescents)

Total N: 262 families (257 adolescents)

Age: mean: NR; range 10 to 14 yrs

Sex (male): $59 \%$

Ethnicity: NR

Country: Germany

Interventions

Number of experimental conditions: 2

Description of Intervention: German adaptation of the lowa Strengthening Families Programme (SFP) 10-14. Manual-based intervention consisting of 7 weekly sessions and 4 booster sessions 4 to 6 months after the last session. Each session includes 8 to 12 families with at least 3 group facilitators. Initially, parent and youth sessions are separate. Youth sessions aim to improve self-efficacy and ability to cope with stress/peer pressure. Parent sessions encourage development of a consistent parenting style and expression of positive affect more openly. In the joint parent-adolescent sessions, dysfunctional communication within the family is addressed and cohesiveness strengthened

Type of intervention: selective (low SES districts)

Focus/target: substance use and behaviour problems

Fidelity: sessions videotaped and adherence rated, $85.5 \%$ adherence to the manual, no inter-rater agreement completed

Dose: NR

Duration/frequency: 7 weekly sessions and 4 booster sessions 4 to 6 mos after

Control: minimal intervention, single parent meeting at which parents received general information on parenting and a leaflet

Outcomes

Length of follow-up: $8 \mathrm{wk}, 6 \& 18 \mathrm{mo}$ (post randomisation)

\section{Alcohol outcomes:}

$\%$ of youth reporting alcohol use in past 30 days

$\%$ of new users

Length of follow-up: 18 mo (post randomisation)

$\%$ of lifetime substance use at $18 \mathrm{mo}$

Funding and Declared Conflicts of Interest
This work was funded by the German Federal Ministry of Education and Research (BMBF; grant 01EL0810). Study authors declared no conflicts of interest 
Baldus 2016 (Continued)

Notes

\section{Risk of bias}

\begin{tabular}{|c|c|c|}
\hline Bias & Authors' judgement & Support for judgement \\
\hline $\begin{array}{l}\text { Random sequence genera- } \\
\text { tion (selection bias) }\end{array}$ & Unclear risk & Not described \\
\hline $\begin{array}{l}\text { Allocation concealment } \\
\text { (selection bias) }\end{array}$ & Unclear risk & Not described \\
\hline $\begin{array}{l}\text { Blinding of participants } \\
\text { and personnel (perfor- } \\
\text { mance bias) } \\
\text { All outcomes }\end{array}$ & High risk & Blinding not possible due to the nature of the intervention \\
\hline $\begin{array}{l}\text { Blinding of outcome as- } \\
\text { sessment (detection bias) } \\
\text { All outcomes }\end{array}$ & High risk & Self-reported \\
\hline $\begin{array}{l}\text { Incomplete outcome data } \\
\text { (attrition bias) } \\
\text { All outcomes }\end{array}$ & Low risk & Attrition low, no differential attrition by group, imputation performed \\
\hline $\begin{array}{l}\text { Selective reporting (re- } \\
\text { porting bias) }\end{array}$ & Unclear risk & $\begin{array}{l}\text { Reported outcomes consistent with protocol and clinical trial registry: ISRCTN } \\
\text { identifier: ISRCTN90251787 }\end{array}$ \\
\hline Other bias & Low risk & Contamination not described and is unlikely \\
\hline Recruitment to cluster & Unclear risk & Not applicable \\
\hline Baseline imbalances & Unclear risk & Not applicable \\
\hline Loss of clusters & Unclear risk & Not applicable \\
\hline Incorrect analysis & Unclear risk & Not applicable \\
\hline $\begin{array}{l}\text { Compatability with indi- } \\
\text { vidually randomised trials } \\
\text { (herd effect) }\end{array}$ & Unclear risk & Not applicable \\
\hline
\end{tabular}

Bauman 2002

$\begin{array}{ll}\text { Methods } & \text { Design: } \mathrm{RCT} \\ & \text { Follow-up: } 3 \& 12 \mathrm{mo} \text { (post intervention) }\end{array}$

Attrition: 19\% (3 mo), 18\% (12 mo)

Unit of randomisation: parent-adolescent dyad ( $96 \%$ mothers)

\begin{tabular}{|c|c|}
\hline Participants & $\begin{array}{l}\text { N of subjects randomised: } \\
\text { Int: NR } \\
\text { Ctrl: NR } \\
\text { Total N: } 1316 \\
\text { N of subjects } 3 \text { \& } 12 \text { mo data combined: } \\
\text { Int: } 531 \\
\text { Ctrl: } 604\end{array}$ \\
\hline
\end{tabular}


Bauman 2002 (Continued)

Total N: 1135

Age: mean: $13.9(\mathrm{SD}=0.9)$; range 12 to $14 \mathrm{yrs}$

Sex (male): $49.3 \%$

Ethnicity: $78 \%$ non-Hispanic White

Country: USA

Interventions

Number of experimental conditions: 2

Description of Intervention: Family Matters involving successive mailings of 4 booklets to families and telephone discussions between health educators and family members. Booklet-1 (identifies and discuss possible consequences of tobacco/alcohol use), booklet-2 (considers non-specific family characteristics known to influence adolescents such as supervision, support, communication skills, time spent together, attachment, conflict reduction), booklet-3 (based on social learning theory; considers tobacco/alcohol-specific variables such as agreement on rules and sanctions for substance use), booklet-4 (based on social inoculation theory; considers variables outside of family that may influence substance use)

Type of intervention: universal

Focus/target: substance use

Fidelity: NR

Dose: $83.4 \%$ completed 1 or more units (booklet plus health educator contact); $61.8 \%$ completed all 4 units

Duration/frequency: average 6 mo

Control: no programme

Outcomes

Length of follow-up: 3 \& 12 mo (post intervention) data combined

\section{Alcohol outcomes:}

$\%$ of youth reporting lifetime alcohol use (over both FUs)

$\%$ of baseline non-users who began to use (over both FUs)

Funding and Declared Conflicts of Interest
This research was supported by Grant No. DA08125 from the National Institute on Drug Abuse, National Institutes of Health, US Department of Health and Human Services No information or declarations about potential conflicts of interest

\section{Notes Abbreviations:}

$\mathrm{FU}=$ follow=up; $\mathrm{RCT}=$ randomised controlled trial; $\mathrm{N}=$ number; Int=intervention; $\mathrm{Ctrl}=$ control; $\mathrm{mo}=$ month $(\mathrm{s}) ; \mathrm{yr}(\mathrm{s})=\mathrm{year}(\mathrm{s}) ; \mathrm{NR}=$ not reported

\section{Risk of bias}

\begin{tabular}{lll}
\hline Bias & Authors' judgement & Support for judgement \\
\hline $\begin{array}{l}\text { Random sequence genera- } \\
\text { tion (selection bias) }\end{array}$ & Unclear risk & Not described \\
\hline $\begin{array}{l}\text { Allocation concealment } \\
\text { (selection bias) }\end{array}$ & Unclear risk & Not described \\
\hline $\begin{array}{l}\text { Blinding of participants } \\
\text { and personnel (perfor- } \\
\text { mance bias) }\end{array}$ & High risk & Blinding not possible due to the nature of the intervention \\
All outcomes & \\
\hline $\begin{array}{l}\text { Blinding of outcome as- } \\
\text { sessment (detection bias) } \\
\text { All outcomes }\end{array}$ & High risk & Self-reported \\
\hline
\end{tabular}


Bauman 2002 (Continued)

Incomplete outcome data Unclear risk Attrition low, differential attrition not described, imputation not described (attrition bias)

All outcomes

\begin{tabular}{lll}
\hline $\begin{array}{l}\text { Selective reporting (re- } \\
\text { porting bias) }\end{array}$ & Unclear risk & No protocol or clinical trial registry available \\
\hline Other bias & Low risk & Contamination not described and is unlikely \\
\hline Recruitment to cluster & Unclear risk & Not applicable \\
\hline Baseline imbalances & Unclear risk & Not applicable \\
\hline Loss of clusters & Unclear risk & Not applicable \\
\hline $\begin{array}{l}\text { Incorrect analysis } \\
\begin{array}{l}\text { Compatability with indi- } \\
\text { vidually randomised trials } \\
\text { (herd effect) }\end{array}\end{array}$ & Unclear risk & Not applicable \\
\hline
\end{tabular}

Bodin 2011

$\begin{array}{ll}\text { Methods } & \text { Design: cluster RCT } \\ & \text { Follow-up: } 12 \& 30 \mathrm{mo} \text { (post randomisation) } \\ & \text { Attrition: } 7.9 \%(12 \mathrm{mo}), 11.6 \%(30 \mathrm{mo}) \\ & \text { Unit of randomisation: school } \\ \text { Clustering effect adjusted: yes }\end{array}$

Participants N of clusters (subjects) randomised:

Int: $20(893)$

Ctrl: 20 (859)

Total N: 40 (1752)

$\mathrm{N}$ of clusters (subjects) at $\mathbf{3 0}$ mo:

Int: 20 (893)

Ctrl: 20 (859)

Total N: 40 (1752)

Age: mean: NR; range 13 to $16 \mathrm{yrs}$

Sex (male): $48.9 \%$

Ethnicity: NR

Country: Sweden

\section{Number of experimental conditions: 2}

Description of Intervention: Orebro Prevention Program (OPP); the current version of OPP encompasses 6 short ( 20 minutes) standardised PowerPoint presentations, administered by trained programme presenters to parents of 13- to 16-year-olds during term regular teacher-parent meetings in grades 7 to 9 . The presentations, which show minimal variation in contents, advise parents to adopt and maintain strict attitudes towards youth drinking, and to not allow their adolescents to have a sip or drink of alcohol at home. Parents are also encouraged to make written agreements on how to prevent their youth from drinking. A summary and the class agreement are subsequently mailed to all parents in the class

Type of intervention: universal

Focus/target: reduce youth drinking 
Bodin 2011 (Continued)

\section{Fidelity: NR}

Dose: Mean number of presentations was $4.7(S D=0.99)$

Duration/frequency: $6 \times 20$ minute presentations during grades 7 to 9 (3 years)

Control: no programme

\section{Outcomes \\ Length of follow-up: $12 \& 30 \mathrm{mo}$ (post randomisation) \\ Alcohol outcomes: \\ Weekly drinking: beverage-specific frequency by quantity measure. Students drinking at least 1 drink at least weekly were coded as weekly drinkers (vs non-weekly drinkers)}

Frequent drunkenness: "How many times /during the last four weeks/have you drunk alcohol to the point that you felt drunk?" Response categories were $0,1,2,3$ to 4, 5 to 7 , and $>8$ times for drunkenness frequency; dichotomised into frequent vs no frequent drunkenness during the last 4 weeks ( $\geq 2$ times, $\geq 1$ time)

Lifetime drunkenness: "How many times have you drunk alcohol to the point that you felt drunk?" $(0,1$, 2 to 4,5 to 10,11 to $20,>20$ ); dichotomised to enable study of programme effects on drunkenness onset

Served alcohol: youth were asked whether they were served alcohol at home with the response alternatives: 0 = "No, my parents do not drink alcohol", 1 = "No, never", 2 = "Yes, sometimes I may have a sip from my parents' glasses", 3 = "Yes, sometimes I can have a glass of alcohol", and 4 = "Yes, sometimes I can have a bottle of wine or a number of beers". Responses were dichotomised ( 0 to $1=0,2$ to $4=1$ )

Funding and Declared Conflicts of Interest
Funding through the Swedish National Institute of Public Health. Study authors declared no conflicts of interest

Notes

Schools were located in diverse municipality types, with intervention and controls distributed fairly evenly within each type

\section{Abbreviations:}

$\mathrm{RCT}=$ randomised controlled trial; $\mathrm{N}=$ number; Int=intervention; $\mathrm{Ctrl}=$ control; $\mathrm{mo}=$ month(s);

$\mathrm{yr}(\mathrm{s})=\mathrm{year}(\mathrm{s}) ; \mathrm{NR}=$ not reported

\section{Risk of bias}

\begin{tabular}{lll}
\hline Bias & Authors' judgement & Support for judgement \\
\hline $\begin{array}{l}\text { Random sequence genera- } \\
\text { tion (selection bias) }\end{array}$ & Low risk & Randomisation sequence was "...generated in advance by coin tossing" \\
\hline $\begin{array}{l}\text { Allocation concealment } \\
\text { (selection bias) }\end{array}$ & Low risk & $\begin{array}{l}\text { "Sealed opaque envelopes with the school's name were mixed and assigned to } \\
\text { groups in blocks of two..." }\end{array}$ \\
\hline $\begin{array}{l}\text { Blinding of participants } \\
\begin{array}{l}\text { and personnel (perfor- } \\
\text { mance bias) }\end{array}\end{array}$ & High risk & Blinding not possible due to the nature of the intervention \\
All outcomes & \\
\hline
\end{tabular}

\begin{tabular}{lll}
$\begin{array}{l}\text { Blinding of outcome as- } \\
\text { sessment (detection bias) } \\
\text { All outcomes }\end{array}$ & High risk & Self-reported \\
\hline $\begin{array}{l}\text { Incomplete outcome data } \\
\text { (attrition bias) } \\
\text { All outcomes }\end{array}$ & High risk & $\begin{array}{l}\text { Attrition low, some differential attrition between groups and by characteristics } \\
\text { (lifetime drunkenness), imputation performed }\end{array}$ \\
\hline
\end{tabular}


Bodin 2011 (Continued)

\begin{tabular}{lll}
$\begin{array}{l}\text { Selective reporting (re- } \\
\text { porting bias) }\end{array}$ & Low risk & $\begin{array}{l}\text { Reported outcomes consistent with the clinical trial registry: clinicaltrials.gov } \\
\text { identifier: NCT01213108 }\end{array}$ \\
\hline Other bias & Low risk & Contamination not described and is unlikely \\
\hline Recruitment to cluster & Low risk & Baseline measurement occurred before randomisation \\
\hline Baseline imbalances & Low risk & $\begin{array}{l}\text { No significant differences between conditions on background measures; near- } \\
\text { significant difference for lifetime drunkenness was observed }(P=0.06)\end{array}$ \\
\hline $\begin{array}{l}\text { Loss of clusters } \\
\text { Incorrect analysis }\end{array}$ & Low risk & No report of loss of entire clusters \\
\hline $\begin{array}{l}\text { Compatability with indi- } \\
\text { vidually randomised trials } \\
\text { (herd effect) }\end{array}$ & Unclear risk & Analysis adjusted for clustering \\
\hline
\end{tabular}

Brody 2006

$\begin{array}{ll}\text { Methods } & \text { Design: cluster RCT } \\ & \text { Follow-up: } 9,18,29,53 \text {, and } 65 \text { mo (post randomisation) } \\ & \text { Attrition: } 15 \%(29 \text { mo cohort } 1) \\ & \text { Unit of randomisation: county } \\ \text { Clustering effect adjusted: no }\end{array}$

\section{Participants}

Cohort 1: follow-up at 29 mo only (Brody 2006)

Cohort 1+2: follow-up at all points (Brody 2010)

$\mathrm{N}$ of clusters (subjects - cohort 1, cohort 1+2) randomised:

Int: $4(307,369)$

Ctrl: $4(214,462)$

Total N: $8(521,831)$

$\mathrm{N}$ of clusters (subjects - cohort 1 ) at $29 \mathrm{mo}$ :

Int: 4 (172)

Ctrl: 4 (133)

Total N: 8 (305)

Age: mean: 11 ; range 10 to $12 \mathrm{yrs}$

Sex (male): NR

Ethnicity: 100\% African American

Country: USA

\section{Number of experimental conditions: 2}

Description of intervention: Strong African American Families Program (SAAF), a prevention programme modelled after existing family skills training interventions, notably SFP, and based on the social development model

7 weekly meetings with separate parent and child skills-building curricula, and a family curriculum; concurrent training sessions for parents and children followed by a joint parent-child session during which families practice newly learned skills

Type of intervention: universal

Focus/target: alcohol use, substance use, and early sexual activity

Fidelity: mean coverage of components $90 \%$ 
Dose: mean attendance 4.4 sessions, $65 \%$ of families took part in 5 or more sessions, $44 \%$ attended all 7 sessions

\section{Duration/frequency: 7 wks}

Control: 3 leaflets via mail - development in early adolescence, stress management, and suggestions for encouraging children to exercise

$\begin{array}{ll}\text { Outcomes } & \text { Length of follow-up: } 9,18,29,53 \text {, and } 65 \text { mo (post randomisation) } \\ \text { Alcohol outcomes: } \\ \text { \% of baseline non-users who began use } \\ \text { Past month alcohol use } \\ \text { Binge drinking occasions in the past month } \\ \text { Alcohol Compositie Index (score } 0 \text { to 3), rate of increase in alcohol use; score consisted of (1) alcohol } \\ \text { initiation, (2) drank at least } 1 \text { drink in the last month, (3) ever drank 3+ drinks at } 1 \text { time }\end{array}$

\begin{tabular}{|c|c|}
\hline \multirow[t]{2}{*}{ Notes } & Abbreviations: \\
\hline & $\begin{array}{l}\mathrm{RCT}=\text { randomised controlled trial; } \mathrm{N}=\text { number; Int=intervention; } \mathrm{Ctrl}=\text { control; } \mathrm{mo}=\text { month(s); } \\
\mathrm{yr}(\mathrm{s})=\operatorname{year}(\mathrm{s}) ; \mathrm{NR}=\text { not reported }\end{array}$ \\
\hline
\end{tabular}

\section{Risk of bias}

\begin{tabular}{lll}
\hline Bias & Authors' judgement & Support for judgement \\
\hline $\begin{array}{l}\text { Random sequence genera- } \\
\text { tion (selection bias) }\end{array}$ & Unclear risk & Not described \\
\hline $\begin{array}{l}\text { Allocation concealment } \\
\text { (selection bias) }\end{array}$ & Unclear risk & Not described \\
\hline
\end{tabular}

Blinding of participants High risk Blinding not possible due to the nature of the intervention
and personnel (perfor-
mance bias)
All outcomes

\begin{tabular}{ll}
\hline Blinding of outcome as- & High risk
\end{tabular}

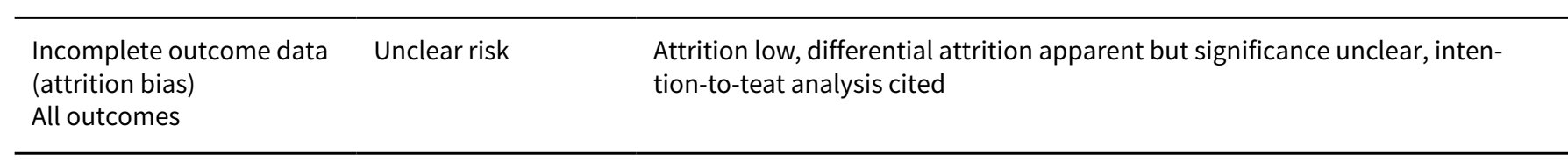

\begin{tabular}{lll}
\hline $\begin{array}{l}\text { Selective reporting (re- } \\
\text { porting bias) }\end{array}$ & Unclear risk & No protocol or clinical trial registry available \\
\hline Other bias & Low risk & $\begin{array}{l}\text { Study authors noted that randomisation at the country level is likely to pre- } \\
\text { vent contamination }\end{array}$ \\
\hline Recruitment to cluster & High risk & Clusters randomised, then participants recruited \\
\hline
\end{tabular}


Brody 2006 (Continued)

\begin{tabular}{lll} 
Baseline imbalances & Low risk & No baseline imbalances \\
\hline Loss of clusters & Low risk & No report of loss of entire clusters \\
\hline Incorrect analysis & Low risk & $\begin{array}{l}\text { Analysis not sufficiently adjusted for clustering; review authors performed an } \\
\text { alternative adjustment }\end{array}$
\end{tabular}

Compatability with indi- Unclear risk

Unable to determine if herd effect exists

vidually randomised trials

(herd effect)

\section{Catalano 1999}

\begin{tabular}{ll}
\hline Methods & Design: $\mathrm{RCT}$ \\
& Follow-up: $6,12 \mathrm{mo} \& 12$ to $15 \mathrm{yrs}$ (post intervention) \\
& Attrition: $6 \%(6 \mathrm{mo}), 8 \%$ (12 mo) parents; $10 \%$ (6 mo), $13 \%$ (12 mo), $15 \%$ (12 to 15 yrs) children \\
& Unit of randomisation: family (parents primarily mothers)
\end{tabular}

Unit of randomisation: family (parents primarily mothers)

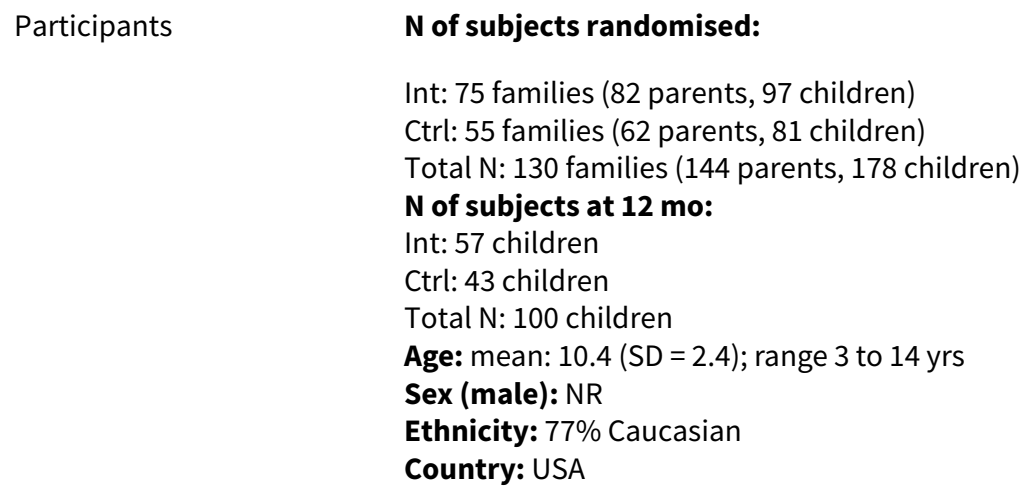

\section{Number of experimental conditions: 2}

Description of Intervention: standard methadone clinic + Focus On Families (FOF) parenting programme. Intervention was delivered through group parent-training workshops at the methadone clinics and through individualised home-based services. FOF: 5 hr family retreat $+32 \times 90$ min meetings (twice weekly). Children attended $12 \times$ curriculum incorporated sessions.

Type of intervention: selective (children of parents attending methadone clinics)

Focus/target: reduce substance use amongst parents, and prevent initiation of drug use in children with a parent in methadone treatment

\section{Fidelity: NR}

Dose: $11(13 \%)$ never attended a single parent training session, although $42(51 \%)$ attended at least $50 \%$ (16) of sessions

Duration/frequency: 9 mo, 1 initial 5 hr workshop + biweekly training sessions, at least 2 phone calls per week. Service providers met 6 times per mo, spending an average of $54 \mathrm{hrs}$ with family during case management period

Control: standard methadone clinic

\section{Alcohol outcomes:}


Drank alcohol (more than a sip) in the previous 6 mo (yes/no)

Length of follow-up: 12 to $15 \mathrm{yrs}$ (post intervention)

\section{Alcohol outcomes:}

Alcohol abuse using the Composite International Diagnostic Interview

Alcohol dependence using the Composite International Diagnostic Interview

Age of onset of alcohol disorder

Funding and Declared Conflicts of Interest
Funding from the National Institute on Drug Abuse. No information or declarations about potential conflicts of interest

\section{Notes \\ Abbreviations: \\ $\mathrm{RCT}=$ randomised controlled trial; $\mathrm{N}=$ number; Int=intervention; $\mathrm{Ctrl}=$ control; $\mathrm{mo}=$ month(s); $\mathrm{yr}(\mathrm{s})=\mathrm{year}(\mathrm{s}) ; \mathrm{NR}=$ not reported}

\section{Risk of bias}

\begin{tabular}{|c|c|c|}
\hline Bias & Authors' judgement & Support for judgement \\
\hline $\begin{array}{l}\text { Random sequence genera- } \\
\text { tion (selection bias) }\end{array}$ & Unclear risk & Not described \\
\hline $\begin{array}{l}\text { Allocation concealment } \\
\text { (selection bias) }\end{array}$ & Unclear risk & Not described \\
\hline $\begin{array}{l}\text { Blinding of participants } \\
\text { and personnel (perfor- } \\
\text { mance bias) } \\
\text { All outcomes }\end{array}$ & High risk & Blinding not possible due to the nature of the intervention \\
\hline $\begin{array}{l}\text { Blinding of outcome as- } \\
\text { sessment (detection bias) } \\
\text { All outcomes }\end{array}$ & High risk & Self-reported \\
\hline $\begin{array}{l}\text { Incomplete outcome data } \\
\text { (attrition bias) } \\
\text { All outcomes }\end{array}$ & Low risk & Attrition low, no differential attrition by group, intention-to-treat analysis cited \\
\hline $\begin{array}{l}\text { Selective reporting (re- } \\
\text { porting bias) }\end{array}$ & Unclear risk & No protocol or clinical trial registry available \\
\hline Other bias & Low risk & Contamination not described and is unlikely \\
\hline Recruitment to cluster & Unclear risk & Not applicable \\
\hline Baseline imbalances & Unclear risk & Not applicable \\
\hline Loss of clusters & Unclear risk & Not applicable \\
\hline Incorrect analysis & Unclear risk & Not applicable \\
\hline $\begin{array}{l}\text { Compatability with indi- } \\
\text { vidually randomised trials } \\
\text { (herd effect) }\end{array}$ & Unclear risk & Not applicable \\
\hline
\end{tabular}


Cordova 2012

$\begin{array}{ll}\text { Methods } & \text { Design: RCT } \\ & \text { Follow-up: } 6,18, \& 30 \text { mo (post randomisation) } \\ & \text { Attrition: } 14.7 \% \text { Family Unidas, } 16.3 \% \text { Community Practice (30 mo) } \\ & \text { Unit of randomisation: adolescent }\end{array}$

Participants
Int: 109
Ctrl: 103
Total N: 212
N of subjects at 30 mo:
Int: US born 65 , foreign born 44
Ctrl: US born 44, foreign born 49
Total N: US born 109, foreign born 103
Age: mean: 13.72 (SD = 0.65); range 12 to 14 yrs
Sex (male): $63.8 \%$ overall; US-born $62.18 \%$, foreign-born $65.59 \%$
Ethnicity: $100 \%$ Hispanic (US-born and foreign-born)
Country: USA

Interventions Number of experimental conditions: 2

Description of Intervention: Familias Unidas; a Hispanic-specific, family-based, substance use (alcohol and drugs) prevention programme. The intended dosage for families randomised to the Familias Unidas intervention included nine 2-hour group sessions and ten 1-hour family visits. Families also attended four 1-hour booster sessions during the follow-up phase, at approximately 10, 16, 22, and 28 months post baseline

Type of intervention: selective (youth with behaviour problems recruited from middle schools)

Focus/target: to target risk and protective factors for alcohol and drug use and to improve family functioning (through developing skills and knowledge of effective parenting)

Fidelity: independent observers rated videotapes of sessions on extensiveness and quality. The average rating was 'considerably/good'

Dose: NR

Duration/frequency: $9 \times 2 \mathrm{hr}$ group sessions, $10 \times 1 \mathrm{hr}$ family sessions, $4 \times 1 \mathrm{hr}$ family booster sessions

Control: Community Practice - given 3 referrals to agencies that provided services for substance use and co-morbid behaviours, in their catchment area

Outcomes

Length of follow-up: 30 mo (post intervention)

\section{Alcohol outcomes:}

$\%$ participants who used alcohol in the past 90 days

Funding and Declared Conflicts of Interest
Funding from the National Insitute on Drug Abuse. No information or declarations about potential conflicts of interest

\begin{tabular}{|c|c|}
\hline \multirow[t]{2}{*}{ Notes } & Abbreviations: \\
\hline & $\begin{array}{l}\mathrm{RCT}=\text { randomised controlled trial; } \mathrm{N}=\text { number; Int=intervention; } \mathrm{Ctrl}=\text { control; } \mathrm{mo}=\text { month(s); } \\
\mathrm{yr}(\mathrm{s})=\mathrm{year}(\mathrm{s}) ; \mathrm{NR}=\text { not reported }\end{array}$ \\
\hline
\end{tabular}

\section{Risk of bias}

\section{Bias}

Authors' judgement Support for judgement 
Cordova 2012 (Continued)
Random sequence genera- Low risk
"An urn randomization program was used..." tion (selection bias)

\begin{tabular}{lll}
\hline $\begin{array}{l}\text { Allocation concealment } \\
\text { (selection bias) }\end{array}$ & Unclear risk & Not described
\end{tabular}

Blinding of participants High risk Blinding not possible due to the nature of the intervention
and personnel (perfor-
mance bias)
All outcomes

\begin{tabular}{lll}
$\begin{array}{l}\text { Blinding of outcome as- } \\
\text { sessment (detection bias) } \\
\text { All outcomes }\end{array}$ & High risk & Self-reported \\
\hline $\begin{array}{l}\text { Incomplete outcome data } \\
\text { (attrition bias) }\end{array}$ & Low risk & $\begin{array}{l}\text { Attrition low, no differential attrition by group, reasons for missing data be- } \\
\text { tween groups not stated }\end{array}$ \\
\hline
\end{tabular}

\begin{tabular}{lll}
\hline $\begin{array}{l}\text { Selective reporting (re- } \\
\text { porting bias) }\end{array}$ & Unclear risk & No protocol or clinical trial registry available. \\
\hline Other bias & Low risk & Contamination not described and is unlikely \\
\hline Recruitment to cluster & Unclear risk & Not applicable \\
\hline Baseline imbalances & Unclear risk & Not applicable \\
\hline Loss of clusters & Unclear risk & Not applicable \\
\hline $\begin{array}{l}\text { Incorrect analysis } \\
\begin{array}{l}\text { Compatability with indi- } \\
\text { vidually randomised trials } \\
\text { (herd effect) }\end{array}\end{array}$ & Unclear risk & Not applicable \\
\hline
\end{tabular}

Dembo 2001

\begin{tabular}{ll}
\hline Methods & Design: RCT \\
& Follow-up: $2,3, \& 4$ yrs (post intervention) \\
& Attrition: $13.7 \%(2 \mathrm{yrs}), 15 \%$ (3 yrs), 24.2\% (4 yrs) \\
& Unit of randomisation: adolescent \\
\hline Participants & N of subjects randomised: \\
& Int: NR \\
Ctrl: NR \\
Total N: 315 \\
N of subjects at last observation at yr 2, 3, or 4: \\
Int: NR \\
Ctrl: NR \\
Total N: 278 \\
Age: mean: mean 14.5; range 11 to 18 yrs \\
Sex (male): $56 \%$ \\
Ethnicity: $56 \%$ Anglo, $41 \%$ African Amercican \\
Country: USA \\
\hline
\end{tabular}


Dembo 2001 (Continued)

Interventions

\section{Number of experimental conditions: 2}

Description of Intervention: Family Empowerment Intervention (FEI) sought to improve family functioning by empowering parents. Project field consultants visited families to work on the following goals: (1) restore family hierarchy (parents, children, etc.); (2) restructure boundaries between parents and children; (3) encourage parents to take greater responsibility for family functioning; (4) increase family structure through implementation of rules and consequences; (5) enhance parenting skills; (6) have parents set limits, expectations, and rules that increase the likelihood the target youth's behavior will improve; (7) improve communication skills among all family members; (8) improve problem-solving skills, particularly among target youth; and (9) where needed, connect the family to other systems (e.g. school, church, community activities)

Type of intervention: selective (youths processed through a juvenile assessment centre after an arrest)

Focus/target: psychosocial functioning among youths

Fidelity: NR

Dose: NR

Duration/frequency: 10 weeks, contact once per week

Control: Extended Services Intervention (ESI) group families received monthly phone contacts from project Research Assistants

\section{Length of follow-up: last observation}

\section{Alcohol outcomes:}

Number of days used alcohol in the past month

Number of times youth got drunk on alcohol in the past year

Funding and Declared Conflicts of Interest
Funding through the National Institute on Drug Abuse. No information or declarations about potential conflicts of interest

\section{Abbreviations:}

$\mathrm{RCT}=$ randomised controlled trial; $\mathrm{N}=$ number; Int=intervention; $\mathrm{Ctrl}=$ control; $\mathrm{mo}=$ month(s); $\mathrm{yr}(\mathrm{s})=\mathrm{year}(\mathrm{s})$; NR=not reported

\section{Risk of bias}

\begin{tabular}{|c|c|c|}
\hline Bias & Authors' judgement & Support for judgement \\
\hline $\begin{array}{l}\text { Random sequence genera- } \\
\text { tion (selection bias) }\end{array}$ & Unclear risk & Not described \\
\hline $\begin{array}{l}\text { Allocation concealment } \\
\text { (selection bias) }\end{array}$ & Unclear risk & Not described \\
\hline $\begin{array}{l}\text { Blinding of participants } \\
\text { and personnel (perfor- } \\
\text { mance bias) } \\
\text { All outcomes }\end{array}$ & High risk & $\begin{array}{l}\text { Blinding of participants and personnel not possible due to the nature of the in- } \\
\text { tervention. }\end{array}$ \\
\hline $\begin{array}{l}\text { Blinding of outcome as- } \\
\text { sessment (detection bias) } \\
\text { All outcomes }\end{array}$ & High risk & $\begin{array}{l}\text { Most outcomes self-reported; blinding of participants and personnel not possi- } \\
\text { ble due to the nature of the intervention. }\end{array}$ \\
\hline
\end{tabular}


Dembo 2001 (Continued)

\begin{tabular}{|c|c|c|}
\hline $\begin{array}{l}\text { Incomplete outcome data } \\
\text { (attrition bias) } \\
\text { All outcomes }\end{array}$ & Low risk & $\begin{array}{l}\text { Attrition low, no differential attrition between groups, no differences in base- } \\
\text { line characteristics between completers and non-completers after accounting } \\
\text { for multiple comparisons }\end{array}$ \\
\hline
\end{tabular}

(attrition bias)

Selective reporting (reporting bias)
High risk

\section{line characteristics between completers and non-completers after accounting}

for multiple comparisons
No protocol or clinical trial registry is available. Numbers of participants in each group and their scores on the outcomes of interest are not presented. Study authors present only results of predicted scores based on last follow-up. Study authors present results on the basis of control vs intervention completers and intervention non-completers. Direct comparison of control vs the entire intervention group is not presented

\begin{tabular}{lll}
\hline Other bias & Low risk & Contamination not described and is unlikely \\
\hline Recruitment to cluster & Unclear risk & Not applicable \\
\hline Baseline imbalances & Unclear risk & Not applicable \\
\hline Loss of clusters & Unclear risk & Not applicable \\
\hline $\begin{array}{l}\text { Incorrect analysis } \\
\begin{array}{l}\text { Compatability with indi- } \\
\text { vidually randomised trials } \\
\text { (herd effect) }\end{array}\end{array}$ & Unclear risk & Not applicable \\
\hline
\end{tabular}

\section{Estrada 2017}

\begin{tabular}{ll}
\hline Methods & Design: $\mathrm{RCT}$ \\
& Follow-up: $6,18, \& 30 \mathrm{mo}$ (post randomisation) \\
& Attrition: $6.6 \%(6 \mathrm{mo}), 9.5 \%(18 \mathrm{mo}), 12.7 \%(30 \mathrm{mo})$ \\
& Unit of randomisation: parent-adolescent dyad \\
\hline Participants & N of subjects randomised: \\
& Int: 376 \\
& Ctrl: 370 \\
& Total N: 746 \\
& N of subjects $\mathbf{3 0}$ mo data: \\
Int: 326 \\
Ctrl: 325 \\
Total N: 651 \\
Age: mean: 13.8 (SD = 0.7); range 12 to 16 yrs \\
Sex (male): $52 \%$ \\
Ethnicity: $100 \%$ Hispanic \\
Country: USA
\end{tabular}

Interventions

\section{Number of experimental conditions: 2}

Description of Intervention: Family Unidas including 8 multi-parent group sessions (10 to 15 parents) and 4 family sessions delivered at school. Group sessions included discussion of adolescent risk factors and ways in which parents can foster parental protective mechanisms, list characteristics of effective family communication, introduce effective discipline to manage behaviour problems, highlight the role of peers, discuss the incidence and consequences of adolescent substance use, identify own attitudes and beliefs in relation to alcohol and risky sexual behaviour, address the role of the school, and list risks for sexually transmitted infections. Family sessions included a family needs assessment and problem-solving barriers to participation, learning communication skills, and practising with adolescents by discussing relevant issues; family conversations about adolescent's peers; parents teach youth the 
skills to manage peer pressure to engage in substance use, and parents communicate the dangers of risky sexual behaviour

Type of intervention: universal

Focus/target: alcohol and drug use, sex without a condom

Fidelity: sessions videotaped and adherence rated on a 7-point scale; average adherence was 3.61 (SD

$=0.56)$, good interrater reliability

Dose: average overall attendance was 6.4 sessions ( $S D=4.2$ ) out of 12 sessions; $12.9 \%$ of participants did not attend any sessions

Duration/frequency: 8 weekly multi-parent group sessions (10 to 15 parents) and 4 family sessions, 3 months' total duration

Control: usual care - HIV risk reduction intervention provided by the Miami-Dade County Public School system, delivered to students; 6 lessons designed to decrease HIV/AIDS and other STIs

\begin{tabular}{ll}
\hline Outcomes & Length of follow-up: $6,18, \& 30$ mo (post randomisation) \\
& Alcohol outcomes: \\
& Whether and how may times alcohol used in past 90 days \\
\hline $\begin{array}{l}\text { Funding and Declared } \\
\text { Conflicts of Interest }\end{array}$ & $\begin{array}{l}\text { This study was funded by the National Institute on Drug Abuse/National Institutes of Health grant R01 } \\
\text { DA025192 and a supplement awarded by the National Institute on Alcohol Abuse and Alcoholism (ad- } \\
\text { ministered by the National Institute on Drug Abuse). No information or declarations about potential } \\
\text { conflicts of interest }\end{array}$
\end{tabular}

Notes

Abbreviations:

$\mathrm{RCT}=$ randomised controlled trial; $\mathrm{N}=$ number; Int=intervention; $\mathrm{Ctrl}=$ control; $\mathrm{mo}=$ =month(s);

$\mathrm{yr}(\mathrm{s})=\mathrm{year}(\mathrm{s})$; NR=not reported

\section{Risk of bias}

\begin{tabular}{|c|c|c|}
\hline Bias & Authors' judgement & Support for judgement \\
\hline $\begin{array}{l}\text { Random sequence genera- } \\
\text { tion (selection bias) }\end{array}$ & Unclear risk & Not described \\
\hline $\begin{array}{l}\text { Allocation concealment } \\
\text { (selection bias) }\end{array}$ & Unclear risk & Not described \\
\hline $\begin{array}{l}\text { Blinding of participants } \\
\text { and personnel (perfor- } \\
\text { mance bias) } \\
\text { All outcomes }\end{array}$ & High risk & Blinding not possible due to the nature of the intervention \\
\hline $\begin{array}{l}\text { Blinding of outcome as- } \\
\text { sessment (detection bias) } \\
\text { All outcomes }\end{array}$ & High risk & Self-reported \\
\hline $\begin{array}{l}\text { Incomplete outcome data } \\
\text { (attrition bias) } \\
\text { All outcomes }\end{array}$ & Low risk & $\begin{array}{l}\text { Attrition low, no differential attrition between groups, missing data accounted } \\
\text { for using full information maximum likelihood }\end{array}$ \\
\hline $\begin{array}{l}\text { Selective reporting (re- } \\
\text { porting bias) }\end{array}$ & Unclear risk & No protocol or clinical trial registry available \\
\hline Other bias & Low risk & Contamination not described and is unlikely \\
\hline Recruitment to cluster & Unclear risk & Not applicable \\
\hline
\end{tabular}


Estrada 2017 (Continued)

\begin{tabular}{|c|c|c|}
\hline Baseline imbalances & Unclear risk & Not applicable \\
\hline Loss of clusters & Unclear risk & Not applicable \\
\hline Incorrect analysis & Unclear risk & Not applicable \\
\hline $\begin{array}{l}\text { Compatability with indi- } \\
\text { vidually randomised trials } \\
\text { (herd effect) }\end{array}$ & Unclear risk & Not applicable \\
\hline
\end{tabular}

Fang 2010

$\begin{array}{ll}\text { Methods } & \text { Design: } \mathrm{RCT} \\ & \text { Follow-up: immediate post-test, } 1 \text { \& } 2 \text { yrs (post randomisation) } \\ & \text { Attrition: } 4 \%(1 \mathrm{yr}), 13.9 \%(2 \mathrm{yrs}) \\ & \text { Unit of randomisation: mother-daughter dyad }\end{array}$

Participants
\[ \begin{array}{l}\text { Nof subjects randomised: } 56 \text { dyads } \\ \text { Ctrl: } 52 \text { dyads } \\ \text { Total N: } 108 \text { dyads } \\ \text { N of subjects at } 2 \text { yrs: } \\ \text { Int: } 56 \text { dyads } \\ \text { Ctrl: } 52 \text { dyads } \\ \text { Total N: } 108 \text { dyads } \\ \text { Age: mean: } 13.10 \text { (SD = 0.96); range } 11 \text { to } 14 \text { yrs } \\ \text { Sex (male): } 0 \% \text { (all female) } \\ \text { Ethnicity: } 100 \% \text { Asian American } \\ \text { Country: USA }\end{array} \]

Interventions

Number of experimental conditions: 2

Description of Intervention: culturally generic substance use prevention programme for Asian American adolescents. 9 -session web-based programme delivered by voice-over narration, animated graphics, and games. Session content involved skill demonstration and interactive exercises requiring joint participation of mother and daughter (1 - my mom and me, 2 - conflict management, 3 - substance use opportunities, 4 - body image, 5 - mood management, 6 - stress management, 7 - problem-solving, 8 social influences, 9 - self-efficacy). A booster session reviewing initial programme content was provided $1 \mathrm{yr}$ after completion of the initial programme

Type of intervention: selective

Focus/target: improve girls' psychological states, strengthen substance use prevention skills, increase mother-daughter interactions, enhance maternal monitoring, and prevent girls' substance use

Fidelity: programme fidelity was ensured by the web-based monitoring system: (1) programme opened at the point at which the participant had previously stopped; (2) the participant could not continue to the next session until completion of the previous one

Dose: 54 of 56 mother-daughter dyads in the intervention group fully attended the initial web-based programme, 53 completed the booster session

Duration/frequency: 9 sessions, planned delivery 1 session per week but average time between baseline and post-intervention survey completion was 6.25 months $(S D=2.25)$

Control: no programme 
Fang 2010 (Continued)

Outcomes

Length of follow-up: immediate post-test, 1 \& 2 yrs (post randomisation), last observation carried forward

\section{Alcohol outcomes:}

30-day alcohol use (occasions of use in past 30 days)

Funding and Declared Conflicts of Interest
Funding through the National Institute on Drug Abuse. No information or declarations about potential conflicts of interest

Notes

\section{Abbreviations:}

$\mathrm{RCT}=$ randomised controlled trial; $\mathrm{N}=$ number; Int=intervention; $\mathrm{Ctrl}=$ control; $\mathrm{mo}=$ month(s); $\mathrm{yr}(\mathrm{s})=\mathrm{year}(\mathrm{s})$; NR=not reported

\section{Risk of bias}

\begin{tabular}{|c|c|c|}
\hline Bias & Authors' judgement & Support for judgement \\
\hline $\begin{array}{l}\text { Random sequence genera- } \\
\text { tion (selection bias) }\end{array}$ & Unclear risk & Not described \\
\hline $\begin{array}{l}\text { Allocation concealment } \\
\text { (selection bias) }\end{array}$ & Unclear risk & Not described \\
\hline $\begin{array}{l}\text { Blinding of participants } \\
\text { and personnel (perfor- } \\
\text { mance bias) } \\
\text { All outcomes }\end{array}$ & High risk & Blinding not possible due to the nature of the intervention \\
\hline $\begin{array}{l}\text { Blinding of outcome as- } \\
\text { sessment (detection bias) } \\
\text { All outcomes }\end{array}$ & High risk & Self-reported \\
\hline $\begin{array}{l}\text { Incomplete outcome data } \\
\text { (attrition bias) } \\
\text { All outcomes }\end{array}$ & Low risk & $\begin{array}{l}\text { Attrition low, equal attrition across both arms; "two mother-daughter dyads } \\
\text { attrited from each arm" }\end{array}$ \\
\hline $\begin{array}{l}\text { Selective reporting (re- } \\
\text { porting bias) }\end{array}$ & Unclear risk & No protocol or clinical trial registry available \\
\hline Other bias & Low risk & Contamination not described and is unlikely \\
\hline Recruitment to cluster & Unclear risk & Not applicable \\
\hline Baseline imbalances & Unclear risk & Not applicable \\
\hline Loss of clusters & Unclear risk & Not applicable \\
\hline Incorrect analysis & Unclear risk & Not applicable \\
\hline $\begin{array}{l}\text { Compatability with indi- } \\
\text { vidually randomised trials } \\
\text { (herd effect) }\end{array}$ & Unclear risk & Not applicable \\
\hline
\end{tabular}


Fosco 2013

\begin{tabular}{ll}
\hline Methods & Design: RCT \\
& Follow-up: $1,2, \& 3$ yrs post randomisation \\
Attrition: $11 \%(2$ yrs $) \& 14 \%(3$ yrs $)$ post randomisation \\
Unit of randomisation: family
\end{tabular}

Interventions

\section{Number of experimental conditions: 2}

Description of Intervention: Family Check-Up (FCU) is a school-based, family-centred intervention that aims to promote family-centred norms and systems for evidence-based family management strategies, and to facilitate identification and referral of students in need of support services. FCU includes the establishment of a family resource centre at each school, staffed by a parent consultant who serves as a resource for all families by providing information about community resources, functioning as a school family liaison, and answering questions. Parent consultants also provide seminars for parents about special topics of interest such as alcohol use. In addition, families involved in FCU participate in a 3-session assessment and feedback process

Type of intervention: universal

Focus/target: youth problem behaviour

Fidelity: NR

Dose: $51 \%$ received consultation from a parent consultant and $42 \%$ received the full FCU intervention; among families receiving the $\mathrm{FCU}, 78 \%$ received additional follow-up assistance after the feedback

Duration/frequency: delivered over 3 years, grades 6 to 8

Control: no programme

Outcomes

Length of follow-up: $1,2, \& 3$ yrs (post randomisation)

\section{Alcohol outcomes:}

Number of alcoholic drinks last month

Funding and Declared Conflicts of Interest
Funded by Grant from the National Institute on Drug Abuse. No information or declarations about potential conflicts of interest

\begin{tabular}{|c|c|}
\hline \multirow[t]{2}{*}{ Notes } & Abbreviations: \\
\hline & $\begin{array}{l}\mathrm{RCT}=\text { randomised controlled trial; } \mathrm{N}=\text { number; Int=intervention; } \text { Ctrl=control; } \mathrm{mo}=\text { month(s); } \\
\mathrm{yr}(\mathrm{s})=\mathrm{year}(\mathrm{s}) ; \mathrm{NR}=\text { not reported }\end{array}$ \\
\hline
\end{tabular}

\section{Risk of bias}

\section{Bias}

Authors' judgement Support for judgement 
Fosco 2013 (Continued)

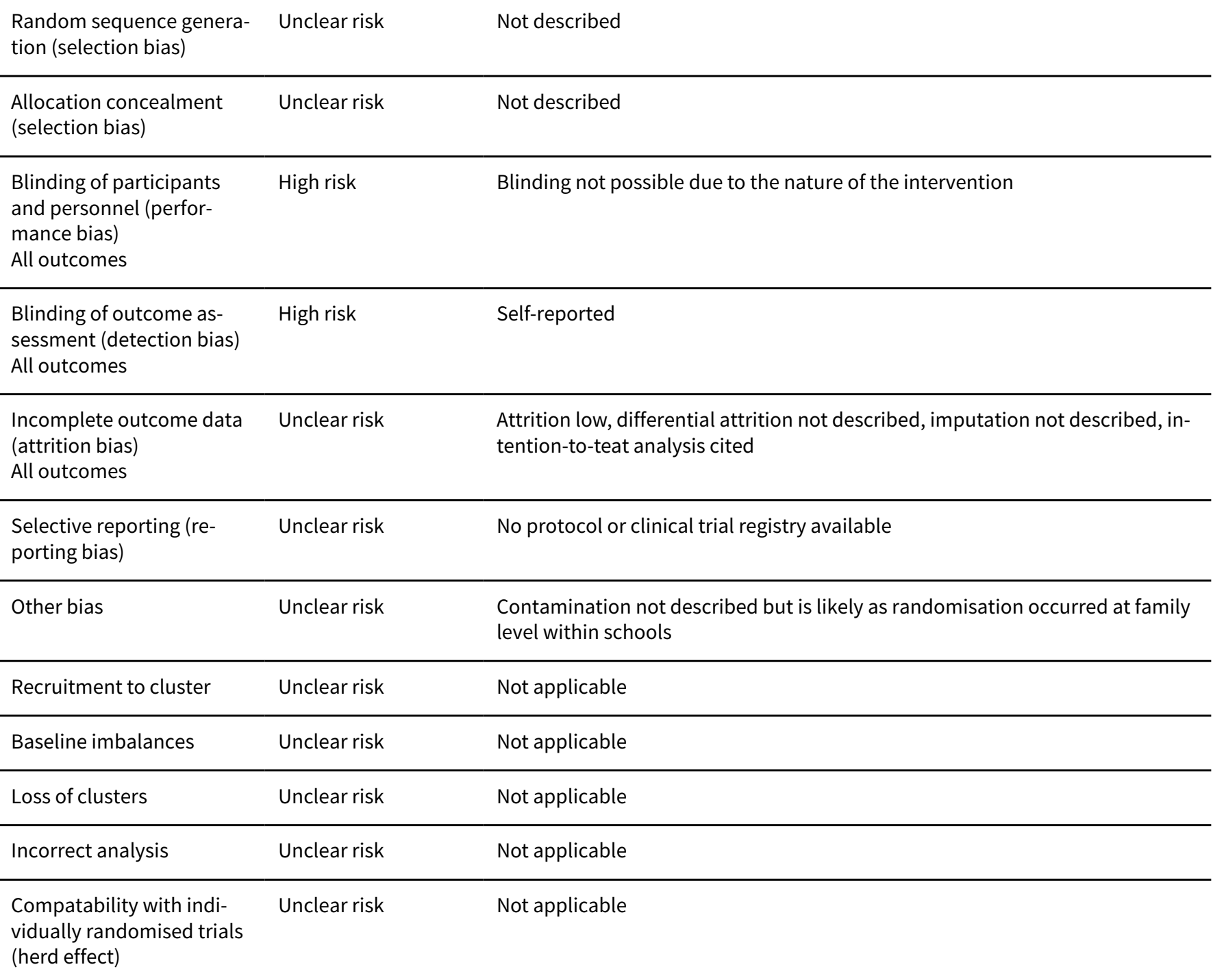

Foxcroft 2017

Methods Design: cluster RCT (16/20 communities randomised, 4 communities assigned: 2 because they were already providing the intervention, and 2 because they were not able to provide the intervention)

Follow-up: 12 \& 24 mo (post randomisation)

Attrition: Int 31\%, Ctrl 22\% (12 mo), Int 48\%, Ctrl 35\% (24 mo)

Unit of randomisation: community

Clustering effect adjusted: yes

Participants N of clusters (subjects) randomised:

Int: 11 randomised, 2 allocated (219 families, 247 adolescents)

Ctrl: 5 randomised, 2 allocated (292 families, 367 adolescents)

Total N: 16 randomised, 4 allocated (511 families, 614 adolescents)

$\mathrm{N}$ of subjects at 24 mo:

Int: 160 families, 177 children

Ctrl: 146 families, 157 children 
Foxcroft 2017 (Continued)

Total N: 306 families, 334 children

Age: mean: $12.1(\mathrm{SD}=1.5)$ intervention, $11.6(\mathrm{SD}=1.4)$ control; range 10 to $14 \mathrm{yrs}$

Sex (male): $63.4 \%$ Int, $55.5 \%$ Ctrl

Ethnicity: NR

Country: Poland

Interventions

\section{Number of experimental conditions: 2}

Description of Intervention: Strengthening Families Program 10-14. SFP10-14 is a video-based programme delivered by trained facilitators that includes parents/guardians and children learning together. The 7-week programme is delivered over 7 sessions. The weekly sessions last 2 hours: in the first hour, parallel groups of children and parents from up to 15 families develop their understanding and skills, led by parent and child group facilitators; in the second hour, parents and children come together in family units to practise the principles they have learned

Type of intervention: universal

Focus/target: USA-developed family-based intervention for preventing alcohol and other drug use and problems amongst young people

Fidelity: NR

Dose: NR

Duration/frequency: $7 \times 2 \mathrm{hr}$ sessions over 7 weeks

Control: Information leaflets for families

Outcomes

Length of follow-up: 12 \& 24 mo

\section{Alcohol outcomes:}

Lifetime alcohol use

Lifetime alcohol use without permission

Past year alcohol use

Past year alcohol use without permission

Past month alcohol use

Past month alcohol use without permission

Lifetime drunkenness

Past year drunkenness

Past month drunkenness

Past month binge drinking

Funding and Declared

Conflicts of Interest
Funded by Polish National Bureau for Drug Prevention and the State Agency for Prevention of Alcohol-Related Problems. The alcohol industry provided support for training and delivery of SFP10-14. Maraton Foundation co-ordinated delivery of SFP10-14 in locations throughout Poland. No funder has had any role in preparation of the manuscript nor in the decision to submit

\section{Notes}

\section{Abbreviations:}

$\mathrm{RCT}=$ randomised controlled trial; $\mathrm{N}=$ number; Int=intervention; $\mathrm{Ctrl}=$ control; $\mathrm{mo}=$ month(s); $\mathrm{yr}(\mathrm{s})=\mathrm{year}(\mathrm{s})$; NR=not reported

\section{Risk of bias}


Foxcroft 2017 (Continued)

\begin{tabular}{|c|c|c|}
\hline Bias & Authors' judgement & Support for judgement \\
\hline $\begin{array}{l}\text { Random sequence genera- } \\
\text { tion (selection bias) }\end{array}$ & High risk & $\begin{array}{l}\text { Community names drawn out of a hat; however, } 4 / 20 \text { communities were not } \\
\text { randomised and their data were retained }\end{array}$ \\
\hline $\begin{array}{l}\text { Allocation concealment } \\
\text { (selection bias) }\end{array}$ & Low risk & Concealed allocation of clusters \\
\hline $\begin{array}{l}\text { Blinding of participants } \\
\text { and personnel (perfor- } \\
\text { mance bias) } \\
\text { All outcomes }\end{array}$ & High risk & Blinding not possible due to the nature of the intervention \\
\hline $\begin{array}{l}\text { Blinding of outcome as- } \\
\text { sessment (detection bias) } \\
\text { All outcomes }\end{array}$ & High risk & Self-reported \\
\hline $\begin{array}{l}\text { Incomplete outcome data } \\
\text { (attrition bias) } \\
\text { All outcomes }\end{array}$ & High risk & Attrition high, lower attrition rates in the control group, imputation performed \\
\hline $\begin{array}{l}\text { Selective reporting (re- } \\
\text { porting bias) }\end{array}$ & High risk & $\begin{array}{l}\text { One primary outcome listed in study protocol (age of first use) was not report- } \\
\text { ed }\end{array}$ \\
\hline Other bias & Low risk & Contamination not described but is unlikely \\
\hline Recruitment to cluster & High risk & Recruitment of individuals to clusters after clusters randomised \\
\hline Baseline imbalances & Low risk & Baseline imbalances accounted for in the analysis \\
\hline Loss of clusters & Low risk & No report of loss of entire clusters \\
\hline Incorrect analysis & Low risk & Analysis adjusted for clustering \\
\hline $\begin{array}{l}\text { Compatability with indi- } \\
\text { vidually randomised trials } \\
\text { (herd effect) }\end{array}$ & Unclear risk & Unable to determine if herd effect exists \\
\hline
\end{tabular}

Furr-Holden 2004

\begin{tabular}{|c|c|}
\hline Methods & $\begin{array}{l}\text { Design: cluster RCT } \\
\text { Follow-up: } 5,6 \text {. or } 7 \text { yrs (post randomisation) } \\
\text { Attrition: } 16 \% \text { ( } 5,6 \text {, or } 7 \text { yrs) } \\
\text { Unit of randomisation: classroom } \\
\text { Clustering effect adjusted: yes }\end{array}$ \\
\hline Participants & 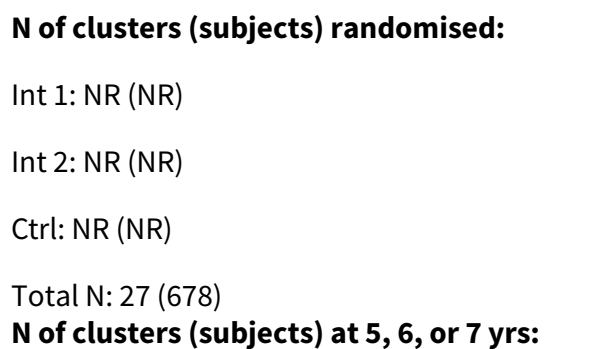 \\
\hline
\end{tabular}


Furr-Holden 2004 (Continued)

Int 1: NR (196)

Int 2: NR (192)

Ctrl: NR (178)

Total N: 27 (566)

Age: mean: $6.2(S D=0.3)$; range 5.3 to $7.7 \mathrm{yrs}$

Sex (male): slightly more than $50 \%$ of the sample

Ethnicity: $85 \%$ to $90 \%$ African American

Country: USA

Interventions Number of experimental conditions: 3

Description of Intervention 1: Family-School Partnership (FSP) intervention, training for teachers/school mental health professionals and other relevant staff in parent-school communication and partnership building, weekly homework learning and communication activities, 9 workshops for parents

Description of Intervention 2: Classroom-Centred (CC) intervention, curricular enhancements, improved classroom behaviour management practices, and supplementary strategies for children not performing adequately

Type of intervention: universal

Focus/target: reduce the risk of early-onset alcohol, inhalant, tobacco, and illegal drug use

Fidelity: FSP: intervenors required to provide details of contacts with parents; live and taped observations of parent workshops were used to determine the extent to which intervention protocols were followed, $\%$ NR

CC: an intervention team member (1) checked classroom setup, (2) observed lessons, and (3) reviewed classroom records, \% NR

Dose: many FSP assigned parents did not complete the intervention, \% NR

Duration/frequency: FSP: 7 parent workshops over consecutive weeks, 2 follow-up workshops (1 every 3 months)

Control: no programme

Outcomes

Length of follow-up: 5, 6, or 7 yrs (post randomisation)

\section{Alcohol outcomes}

Alcohol use without parental permission

Funding and Declared Conflicts of Interest
Funded by Grant from the National Institute on Drug Abuse. No information or declarations about potential conflicts of interest

\section{Notes}

\section{Abbreviations:}

$\mathrm{RCT}=$ randomised controlled trial; $\mathrm{N}=$ number; Int=intervention; $\mathrm{Ctrl}=$ control; $\mathrm{mo}=$ month(s); $\mathrm{yr}(\mathrm{s})=\mathrm{year}(\mathrm{s})$; NR=not reported; FSP= Family-school partnership, $C \mathrm{C}=$ Classroom-centered

\section{Risk of bias}

\begin{tabular}{lll}
\hline Bias & Authors' judgement & Support for judgement \\
\hline $\begin{array}{l}\text { Random sequence genera- } \\
\text { tion (selection bias) }\end{array}$ & Unclear risk & Not described \\
\hline $\begin{array}{l}\text { Allocation concealment } \\
\text { (selection bias) }\end{array}$ & Unclear risk & Not described \\
\hline \hline
\end{tabular}


Furr-Holden 2004 (Continued)

Blinding of participants High risk Blinding not possible due to the nature of the intervention and personnel (performance bias)

All outcomes

\begin{tabular}{|c|c|c|}
\hline $\begin{array}{l}\text { Blinding of outcome as- } \\
\text { sessment (detection bias) } \\
\text { All outcomes }\end{array}$ & High risk & Self-reported \\
\hline $\begin{array}{l}\text { Incomplete outcome data } \\
\text { (attrition bias) } \\
\text { All outcomes }\end{array}$ & Low risk & $\begin{array}{l}\text { Attrition low, no differential attrition between by groups or characteristics, } \\
\text { outcome data based on data from all available outcome assessments }\end{array}$ \\
\hline $\begin{array}{l}\text { Selective reporting (re- } \\
\text { porting bias) }\end{array}$ & Low risk & Prespecificed outcome measures reported \\
\hline Other bias & Low risk & Contamination not described but is unlikely \\
\hline Recruitment to cluster & Low risk & $\begin{array}{l}\text { Classrooms within schools were randomised and students then entering were } \\
\text { randomised to classrooms; parental awareness of the study on enrolment is } \\
\text { not known but bias on this basis is unlikely }\end{array}$ \\
\hline Baseline imbalances & Low risk & Baseline imbalances accounted for in analysis \\
\hline Loss of clusters & Low risk & No report of loss of entire clusters \\
\hline Incorrect analysis & Low risk & Analysis adjusted for clustering \\
\hline
\end{tabular}

Compatability with indi-

Unclear risk

Unable to determine if herd effect exists

vidually randomised trials

(herd effect)

\section{Haggerty 2007}

\begin{tabular}{ll}
\hline Methods & Design: $\mathrm{RCT}$ \\
& Follow-up: $24 \mathrm{mo}$ (post intervention) \\
& Attrition: $8 \%(24 \mathrm{mo})$ \\
& Unit of randomisation: family
\end{tabular}

\section{Participants}

\section{$\mathrm{N}$ of subjects randomised:}

Int-1: 107

Int-2: 118

Ctrl: 106

Total N: 331

$\mathrm{N}$ of subjects at $24 \mathrm{mo}$ :

Int-1: 73

Int-2: 84

Ctrl: 79

Total N: 236

Age: mean: 13.7

Sex (male): $51 \%$

Ethnicity: $49.2 \%$ African American, 51\% White

Country: USA 
Haggerty 2007 (Continued)

Interventions

\section{Number of experimental conditions: 3}

Description of Intervention 1: Parents Who Care (PWC) is a programme based on social development model. The PWC programme includes a video and a workbook. Chapters include the following: Roles - relating to your teen, Risks - Identifying and reducing them, Protection - Bonding with your teen to strengthen resilience, Tools - Working with your family to solve problems, Involvement - allowing everyone to contribute, Policies - Setting family policies on health and safety issue, Supervision - Supervising without invading. Participants of Parents Who Care - Self-Administered with telephone support (PWC-SA) is provided with the video and workbook, along with a checklist of activities and follow-up with phone calls

Description of Intervention 2: Parents Who Care - Parent and Adolescent format (PWC-PA) includes the content of PWC as described previously. Participants of PWC-PA meet for 7 sessions with their adolescent. In these sessions, they view the curriculum, practice specific skills separately, practice skills in structured family interaction tasks, and are given homework

Type of intervention: universal

Focus/target: substance use and other problem behaviours

Fidelity: PWC-SA: difficult to assess as self-directed, parents contacted and asked to report activities completed, mean task completion 74\%; PWC-PA: parent and teen group intervention guided by a structured written curriculum, with each family session independently observed and rate; overall content covered per group ranged from $75.5 \%$ to $88.3 \%$, with an average of $82.3 \%$

Dose: PWC-SA: 8 (7.5\%) families did not complete any tasks; PWC-PA: $26(22 \%)$ families did not attend any sessions

Duration/frequency: 7 to $10 \mathrm{wks}$

Control: no programme

\begin{tabular}{ll}
\hline Outcomes & Length of follow-up: 24 mo (post intervention) \\
& Alcohol outcomes \\
& Alcohol use initiation \\
\hline $\begin{array}{l}\text { Funding and Declared } \\
\text { Conflicts of Interest }\end{array}$ & $\begin{array}{l}\text { This paper was supported by Grant \# R01DA121645-05 from the National Institute on Drug Abuse. No in- } \\
\text { formation or declarations about potential conflicts of interest }\end{array}$ \\
\hline Notes & Abbreviations: \\
& $\begin{array}{l}\text { RCT=randomised controlled trial; N=number; Int=intervention; Ctrl=control; mo=month(s); } \\
\text { yr(s)=year(s); NR=not reported; PWC-SA: Parent's Who Care - Self-administered; PWC-PA: Parent's Who } \\
\text { Care - Parent and adolescent }\end{array}$
\end{tabular}

\section{Risk of bias}

\begin{tabular}{lll}
\hline Bias & Authors' judgement & Support for judgement \\
\hline $\begin{array}{l}\text { Random sequence genera- } \\
\text { tion (selection bias) }\end{array}$ & Unclear risk & Not described \\
\hline $\begin{array}{l}\text { Allocation concealment } \\
\text { (selection bias) }\end{array}$ & Unclear risk & Not described \\
\hline $\begin{array}{l}\text { Blinding of participants } \\
\text { and personnel (perfor- } \\
\begin{array}{l}\text { mance bias) } \\
\text { All outcomes }\end{array}\end{array}$ & High risk & Blinding not possible due to the nature of the intervention \\
\hline
\end{tabular}


Haggerty 2007 (Continued)

Blinding of outcome as- High risk Self-reported sessment (detection bias)

All outcomes

\begin{tabular}{lll}
$\begin{array}{l}\text { Incomplete outcome data } \\
\text { (attrition bias) } \\
\text { All outcomes }\end{array}$ & Low risk & Attrition low, no differential attrition by group or characteristics \\
\hline $\begin{array}{l}\text { Selective reporting (re- } \\
\text { porting bias) }\end{array}$ & Unclear risk & No protocol or clinical trial registry available \\
\hline Other bias & Low risk & Not applicable \\
\hline Recruitment to cluster & Unclear risk & Not applicable \\
\hline Baseline imbalances & Unclear risk & Not applicable \\
\hline $\begin{array}{l}\text { Loss of clusters } \\
\text { Incorrect analysis }\end{array}$ & Unclear risk & Not applicable \\
\hline $\begin{array}{l}\text { Compatability with indi- } \\
\text { vidually randomised trials } \\
\text { (herd effect) }\end{array}$ & Unclear risk & Not applicable \\
\hline
\end{tabular}

\section{Koning 2009}

Methods Design: cluster RCT

Follow-up: 10, 22, 34, 50 mo (post randomisation)

Attrition: $26.4 \%$ (from randomisation to $22 \mathrm{mo}$ )

Unit of randomisation: school

Clustering effect adjusted: yes

\section{Participants N of clusters (subjects) randomised:}

Int 1: NR (801)

Int 2: NR (942)

Int 3: NR (812)

Ctrl: NR (935)

Total N: $19(3490)$

\section{$\mathrm{N}$ of clusters (subjects) at $22 \mathrm{mo}$ :}

Int 1: NR (608)

Int 2: NR (675)

Int 3: NR (588)

Ctrl: NR (699)

Total N: 19 (2570)

$\mathrm{N}$ of clusters (subjects) at $34 \mathrm{mo}$ :

Int 1: NR (603)

Int 2: NR (671)

Int 3: NR (582)

Ctrl: NR (677)

Total N: 19 (2533)

Age: mean: $12.66(\mathrm{SD}=0.49)$

Sex (male): $51 \%$ 
Koning 2009 (Continued)

\author{
Ethnicity: NR \\ Country: Netherlands
}

Interventions

\title{
Number of experimental conditions: 4
}

Description of Intervention 1: Parent Intervention (PI), modelled off the Swedish Orebro Prevention Program. Targets parental rules for children's alcohol use. Includes (1) 20 min parent meeting about the adverse effects of alcohol use \& negative effects of permissive parental attitudes, given by expert on alcohol use, (2) meeting of parents of the same class + mentor of that class to discuss shared rules, and (3) information leaflet with a summary sent home

Description of Intervention 2: Student Intervention (SI), digital alcohol programme based on alcohol module of Healthy School and Drugs Dutch prevention programme. Includes 4 lessons of digital alcohol programme. Targets students' abilities to develop a healthy attitude towards alcohol use and to train their refusal skills

Description of Intervention 3: PI+SI - schools carried out a combination of the parent intervention and the student intervention

Type of intervention: universal

Focus/target: reducing alcohol use

Fidelity: NR

Dose: NR

Duration/frequency: 2 mo

Control: no programme/SI only

\section{Outcomes}

Length of follow-up: 10 \& 22 mo (post intervention)

\section{Alcohol outcomes:}

Onset of weekly drinking

Onset of heavy weekly drinking

Frequency of drinking in the last month

Glasses of alcohol consumption per week - non-users

Glasses of alcohol consumption per week - users

Length of follow-up: 34 mo (post intervention)

\section{Alcohol outcomes:}

Onset of weekly drinking

Onset of heavy weekly drinking

Glasses of alcohol consumption per week - non-users

Glasses of alcohol consumption per week - users

Length of follow-up: 50 mo (post intervention)

\section{Alcohol outcomes:}

Onset of heavy weekly drinking

Amount of drinking on weekends 
Koning 2009 (Continued)

Funding and Declared Conflicts of Interest
This study was funded by grant numbers 6220,0021 from the Dutch Health Care Research Organization (Z.O.N.-M.W). Study authors declare no conflicts of interest

Notes

\section{Abbreviations:}

$\mathrm{RCT}=$ randomised controlled trial; $\mathrm{N}=$ number; Int=intervention; $\mathrm{Ctrl}=$ control; $\mathrm{mo}=$ month(s); $\mathrm{yr}(\mathrm{s})=\mathrm{year}(\mathrm{s})$; NR=not reported

\section{Risk of bias}

\begin{tabular}{|c|c|c|}
\hline Bias & Authors' judgement & Support for judgement \\
\hline $\begin{array}{l}\text { Random sequence genera- } \\
\text { tion (selection bias) }\end{array}$ & Unclear risk & Not described \\
\hline $\begin{array}{l}\text { Allocation concealment } \\
\text { (selection bias) }\end{array}$ & Low risk & Central randomisation \\
\hline $\begin{array}{l}\text { Blinding of participants } \\
\text { and personnel (perfor- } \\
\text { mance bias) } \\
\text { All outcomes }\end{array}$ & High risk & Blinding not possible due to the nature of the intervention \\
\hline $\begin{array}{l}\text { Blinding of outcome as- } \\
\text { sessment (detection bias) } \\
\text { All outcomes }\end{array}$ & High risk & Self-reported \\
\hline $\begin{array}{l}\text { Incomplete outcome data } \\
\text { (attrition bias) } \\
\text { All outcomes }\end{array}$ & High risk & $\begin{array}{l}\text { Attrition high, differential attrition by group apparent but significance not } \\
\text { clear, some differential attrition by characteristics, imputation performed }\end{array}$ \\
\hline $\begin{array}{l}\text { Selective reporting (re- } \\
\text { porting bias) }\end{array}$ & Unclear risk & No protocol or clinical trial registry available \\
\hline Other bias & Low risk & Contamination not described and is unlikely \\
\hline Recruitment to cluster & High risk & Recruitment of individuals to clusters after clusters randomised \\
\hline Baseline imbalances & Low risk & Baseline imbalances accounted for in analysis \\
\hline Loss of clusters & High risk & $\begin{array}{l}\text { After randomisation, } 1 \text { school could not participate for reasons unrelated to } \\
\text { the study; this school was randomised originally to the control condition }\end{array}$ \\
\hline Incorrect analysis & Low risk & Analysis adjusted for clustering \\
\hline $\begin{array}{l}\text { Compatability with indi- } \\
\text { vidually randomised trials } \\
\text { (herd effect) }\end{array}$ & Unclear risk & Unable to determine if herd effect exists \\
\hline
\end{tabular}

Liddle 2008

\begin{tabular}{ll}
\hline Methods & Design: $\mathrm{RCT}$ \\
& Follow-up: immediate post-test, $6 \& 12 \mathrm{mo}$ (post intervention) \\
& Attrition: $45 \%$ (immediate post-test), $46 \%$ (6 mo), 39\% (12 mo) \\
& Unit of randomisation: adolescent
\end{tabular}


Liddle 2008 (Continued)

Participants

\section{N of subjects randomised:}

Int 1:112

Int 2: 112

Total N: 224

$\mathrm{N}$ of subjects at $12 \mathrm{mo}$ :

Int 1: 39

Int 2: 49

Total N: 88

Age: mean: $15.4(\mathrm{SD}=1.23)$

Sex (male): $81 \%$

Ethnicity: $72 \%$ African American, 18\% White, 10\% Hispanic

Country: USA

\section{Number of experimental conditions: 2}

Description of Intervention 1: Multi-Dimensional Family Therapy (MDFT) included a 60 to 90 min weekly session with the family. Therapists work simultaneously in 4 domains depending on the profile of the family (adolescent domain, parent domain, interactional domain, extrafamilial domain). Each session includes a family session and a session with the parent alone and/or a session with the adolescent alone

Description of Intervention 2: Cognitive-Behavioural therapy (CBT), 60 to 90 min weekly session with individual adolescent. Problems are prioritised and a treatment plan is developed. Parents attended the first 2 sessions to support adolescent participation. Next, the aim is to increase coping competence and reduce behaviours that threaten safety, health, and quality of life. Typical strategies include (1) providing information and education; (2) contingency contracting; (3) self-monitoring; (4) problem-solving training; (5) communication skills training; (6) identifying cognitive distortions; (7) increasing healthy recreational activities; and (8) providing homework assignments

Type of intervention: indicated

Focus/target: harm reduction from substance use

Fidelity: raters blind to condition; results indicate each condition used techniques in accordance with their model and avoided interventions prescribed in the other treatment

Dose: median number of sessions of therapy was 8; no differences between groups in treatment dosage or total time in treatment

Duration/frequency: both interventions have 60 to 90 minute weekly sessions

Control: not applicable
Length of follow-up: 12 mo (post intervention)

\section{Alcohol outcomes}

Number of days alcohol used in the previous 30 days

Funding and Declared Grant from the National Institute on Drug Abuse. Study authors declared no conflicts of interest

\section{Abbreviations:}

$\mathrm{RCT}=$ randomised controlled trial; $\mathrm{N}=$ number; Int=intervention; $\mathrm{Ctrl}=$ control; $\mathrm{mo}=$ month(s); $\operatorname{yr}(\mathrm{s})=\mathrm{year}(\mathrm{s}) ; \mathrm{NR}=$ not reported

\section{Risk of bias}


Liddle 2008 (Continued)

\begin{tabular}{|c|c|c|}
\hline Bias & Authors' judgement & Support for judgement \\
\hline $\begin{array}{l}\text { Random sequence genera- } \\
\text { tion (selection bias) }\end{array}$ & Unclear risk & Not described \\
\hline $\begin{array}{l}\text { Allocation concealment } \\
\text { (selection bias) }\end{array}$ & Unclear risk & Not described \\
\hline $\begin{array}{l}\text { Blinding of participants } \\
\text { and personnel (perfor- } \\
\text { mance bias) } \\
\text { All outcomes }\end{array}$ & High risk & $\begin{array}{l}\text { Blinding of participants and personnel not possible due to the nature of the in- } \\
\text { tervention. }\end{array}$ \\
\hline $\begin{array}{l}\text { Blinding of outcome as- } \\
\text { sessment (detection bias) } \\
\text { All outcomes }\end{array}$ & High risk & $\begin{array}{l}\text { Self-reported; blinding of participants and personnel not possible due to the } \\
\text { nature of the intervention }\end{array}$ \\
\hline $\begin{array}{l}\text { Incomplete outcome data } \\
\text { (attrition bias) } \\
\text { All outcomes }\end{array}$ & High risk & $\begin{array}{l}\text { Attrition high, no differential attrition between groups, missing data accom- } \\
\text { modated by using all available data through full information maximum likeli- } \\
\text { hood estimation and the expectation-maximisation algorithm }\end{array}$ \\
\hline $\begin{array}{l}\text { Selective reporting (re- } \\
\text { porting bias) }\end{array}$ & Unclear risk & No protocol or clinical trial registry available \\
\hline Other bias & Low risk & Contamination not described and is unlikely \\
\hline Recruitment to cluster & Unclear risk & Not applicable \\
\hline Baseline imbalances & Unclear risk & Not applicable \\
\hline Loss of clusters & Unclear risk & Not applicable \\
\hline Incorrect analysis & Unclear risk & Not applicable \\
\hline $\begin{array}{l}\text { Compatability with indi- } \\
\text { vidually randomised trials } \\
\text { (herd effect) }\end{array}$ & Unclear risk & Not applicable \\
\hline
\end{tabular}

Linakis 2013

$\begin{array}{ll}\text { Methods } & \text { Design: } \mathrm{RCT} \\ & \text { Follow-up: } 6 \text { mo (post intervention) } \\ & \text { Attrition: } 11 \% \text { parents, } 6 \% \text { adolescents }(6 \mathrm{mo}) \\ & \text { Unit of randomisation: parent-adolescent dyad }\end{array}$

Participants

\section{$\mathrm{N}$ of subjects randomised:}

Int: 44

Ctrl: 45

Total N: 89

$\mathrm{N}$ of subjects at 6 mo:

Int: 39

Ctrl: 41

Total N: 80

Age: mean: $13(\mathrm{SD}=0.83)$ 
Linakis 2013 (Continued)

\author{
Sex (male): $49 \%$ \\ Ethnicity: $71 \%$ White \\ Country: USA
}

\begin{abstract}
Interventions Number of experimental conditions: 2
Description of Intervention: Brief Prevention Intervention (BPI) in the paediatric emergency department waiting room. 25 to 40 min session targeting parental monitoring and the importance of parent-adolescent communication. Session was run by a counsellor and used motivational interviewing. Adolescents were engaged in the sessions to promote their involvement in the monitoring and communication process. Also received booster telephone calls and mail-out information
\end{abstract}

Type of intervention: universal

Focus/target: delay or prevent initiation of alcohol use

Fidelity: weekly supervision and audiotape review were conducted, using the MITI-3 five-point scale rating system on MI principles and methods (Evocation, Collaboration, Autonomy/Support, Direction, Empathy); over $97 \%$ of supervisor-rated tapes met the performance criterion (score $\geq 3$ ) on each item

Dose: $100 \%$ completed the intervention, and $53 \%$ and $38 \%$ of parents in the intervention completed the first \& second telephone booster contacts, respectively

Duration/frequency: 3 mo, sessions 25 to $40 \mathrm{~min}$, mail-out/telephone call at $1 \& 3$ mo post discharge

Control: Enhanced Standard Care (ESC), regular medical care, assessment battery \& mail-out about local family opportunities and the influence parents have on their adolescents' alcohol use)

\begin{tabular}{ll}
\hline Outcomes & Length of follow-up: 6 mo (post intervention) \\
& Alcohol outcomes: \\
& Number of days alcohol used in previous 30 days \\
& Number of days consumed 4 or more drinks in previous 30 days \\
\hline $\begin{array}{l}\text { Funding and Declared } \\
\text { Conflicts of Interest }\end{array}$ & NIAAA Grant. No information or declarations about potential conflicts of interest \\
\hline Notes & Abbreviations: \\
& $\begin{array}{l}\text { RCT=randomised controlled trial; } N=\text { number; Int=intervention; Ctrl=control; mo=month(s); } \\
\mathrm{yr}(\mathrm{s})=y e a r(s) ; N R=n o t \text { reported }\end{array}$
\end{tabular}

\title{
Risk of bias
}

\begin{tabular}{lll}
\hline Bias & Authors' judgement & Support for judgement \\
\hline $\begin{array}{l}\text { Random sequence genera- } \\
\text { tion (selection bias) }\end{array}$ & Unclear risk & Not described \\
\hline $\begin{array}{l}\text { Allocation concealment } \\
\text { (selection bias) }\end{array}$ & Unclear risk & Not described \\
\hline $\begin{array}{l}\text { Blinding of participants } \\
\text { and personnel (perfor- } \\
\text { mance bias) } \\
\text { All outcomes }\end{array}$ & High risk & Blinding not possible due to the nature of the intervention \\
\hline
\end{tabular}

$\begin{array}{lll}\text { Blinding of outcome as- } & \text { High risk } & \text { Self-reported } \\ \text { sessment (detection bias) } & & \end{array}$


Linakis 2013 (Continued)

All outcomes

\begin{tabular}{|c|c|c|}
\hline $\begin{array}{l}\text { Incomplete outcome data } \\
\text { (attrition bias) } \\
\text { All outcomes }\end{array}$ & Low risk & Attrition low, no differential attrition between groups \\
\hline $\begin{array}{l}\text { Selective reporting (re- } \\
\text { porting bias) }\end{array}$ & Unclear risk & No protocol or clinical trial registry available \\
\hline Other bias & Low risk & Contamination not described and is unlikely \\
\hline Recruitment to cluster & Unclear risk & Not applicable \\
\hline Baseline imbalances & Unclear risk & Not applicable \\
\hline Loss of clusters & Unclear risk & Not applicable \\
\hline Incorrect analysis & Unclear risk & Not applicable \\
\hline $\begin{array}{l}\text { Compatability with indi- } \\
\text { vidually randomised trials } \\
\text { (herd effect) }\end{array}$ & Unclear risk & Not applicable \\
\hline
\end{tabular}

Loveland-Cherry 1999

\begin{tabular}{ll}
\hline Methods & Design: RCT \\
& Follow-up: immediate post-test, 1, 2, 3, \& 4 yrs (post randomisation) \\
& Attrition: $19 \%(4 \mathrm{yrs})$ \\
& Unit of randomisation: family \\
\hline
\end{tabular}

Participants N of subjects randomised:

Int: NR

Ctrl: NR

Total N: 892

$\mathrm{N}$ of subjects at 4 yrs:

Int: NR

Ctrl: NR

Total N: 723

Age: mean: NR (4th grade)

Sex (male): $46 \%$

Ethnicity: $86 \%$ White

Country: USA

Number of experimental conditions: 2

Description of Intervention: general parenting skills and family functioning: Children and Parent Relations (CAPR). Programme (in 4th grade) carried out in 3 home sessions, each 1 hr long, family meetings, and follow-up telephone calls. Booster was added (in 7 th grade)

Type of intervention: universal

Focus/target: decrease alcohol use/misuse

Fidelity: NR

Dose: NR 
Loveland-Cherry 1999 (Continued)

Duration/frequency: 3 sessions in 3 mo

Control: no programme

\begin{tabular}{|c|c|c|}
\hline Outcomes & \multicolumn{2}{|c|}{$\begin{array}{l}\text { Length of follow-up: immediate post-test, } 1,2,3, \& 4 \text { yrs (post randomisation) } \\
\text { Alcohol outcomes: } \\
\text { Alcohol use per week over the last } 12 \text { mo (quantity-frequency) } \\
\text { Alcohol misuse per week over the last } 12 \text { mo }\end{array}$} \\
\hline $\begin{array}{l}\text { Funding and Declared } \\
\text { Conflicts of Interest }\end{array}$ & \multicolumn{2}{|c|}{$\begin{array}{l}\text { This work was funded by National Institute on Alcohol Abuse and Alcoholism, National Institutes of } \\
\text { Health, grant RO } 1 \text { AA0844 7. No information or declarations about potential conflicts of interest }\end{array}$} \\
\hline \multicolumn{3}{|l|}{ Notes } \\
\hline \multicolumn{3}{|l|}{ Risk of bias } \\
\hline $\begin{array}{l}\text { Random sequence genera- } \\
\text { tion (selection bias) }\end{array}$ & Unclear risk & Not described \\
\hline $\begin{array}{l}\text { Allocation concealment } \\
\text { (selection bias) }\end{array}$ & Unclear risk & Not described \\
\hline $\begin{array}{l}\text { Blinding of participants } \\
\text { and personnel (perfor- } \\
\text { mance bias) } \\
\text { All outcomes }\end{array}$ & High risk & Blinding not possible due to the nature of the intervention \\
\hline $\begin{array}{l}\text { Blinding of outcome as- } \\
\text { sessment (detection bias) } \\
\text { All outcomes }\end{array}$ & High risk & Self-reported \\
\hline $\begin{array}{l}\text { Incomplete outcome data } \\
\text { (attrition bias) } \\
\text { All outcomes }\end{array}$ & High risk & $\begin{array}{l}\text { Attrition low in terms of completers but high based on those with complete } \\
\text { data; complete data used in the analysis; differential attrition not described }\end{array}$ \\
\hline $\begin{array}{l}\text { Selective reporting (re- } \\
\text { porting bias) }\end{array}$ & Unclear risk & No protocol or clinical trial registry available \\
\hline Other bias & Low risk & Contamination not described and is unlikely \\
\hline Recruitment to cluster & Unclear risk & Not applicable \\
\hline Baseline imbalances & Unclear risk & Not applicable \\
\hline Loss of clusters & Unclear risk & Not applicable \\
\hline Incorrect analysis & Unclear risk & Not applicable \\
\hline $\begin{array}{l}\text { Compatability with indi- } \\
\text { vidually randomised trials } \\
\text { (herd effect) }\end{array}$ & Unclear risk & Not applicable \\
\hline
\end{tabular}


Mares 2016

\begin{tabular}{ll}
\hline Methods & Design: cluster RCT \\
& Follow-up: 18 mo (post randomisation) \\
Attrition: $15.9 \%$ mothers, $17.5 \%$ children (18 mo) & Unit of randomisation: school \\
& Clustering effect adjusted: yes \\
\hline Participants & N of clusters (subjects) randomised: \\
& Int: NR (669) \\
Ctrl: NR (680) & Total N: 807 (1349) \\
& N of clusters (subjects) at 18 mo: \\
& Int: NR (540) \\
Ctrl: NR (601) & Total N: NR (1141) \\
Age: mean: 12.15 (SD = 0.47); range 11 to 12 yrs \\
Sex (male): $51.1 \%$ \\
Ethnicity: $97.1 \%$ Dutch \\
Country: Netherlands
\end{tabular}

Interventions

\section{Number of experimental conditions: 2}

Description of Intervention: the programme, "In control: No alcohol!", comprised 5 modules, sent to families by mail over 5 months, 1 module every 4 weeks, starting in May/June 2011. Each module was a magazine with information for parents and several attractive assignments for parents and children to go over together

Type of intervention: universal

Focus/target: delay initiation of alcohol use

Fidelity: NR

Dose: of families participating in the intervention, $66.5 \%$ of the children and $62.7 \%$ of the mothers read at least 3 of 5 activity modules

Duration/frequency: 1 module every 4 weeks over 5 mo

Control: brochure including facts about alcohol

Outcomes Length of follow-up: $18 \mathrm{mo}$ (post randomisation)

\section{Alcohol outcomes:}

Alcohol initiation - ever drank alcohol (at least 1 glass)

Alcohol initiation - ever drank alcohol (at least 1 sip)

Funding and Declared Conflicts of Interest
Funded by ZonMw, the Netherlands Organistion for Health Research and Development. Declaration of no connection with the alcohol, tobacco, or gaming industries. Study authors employed by institute that developed the intervention programme

\section{Notes}

\section{Risk of bias}

Bias Authors' judgement Support for judgement


Mares 2016 (Continued)

\begin{tabular}{|c|c|c|}
\hline $\begin{array}{l}\text { Random sequence genera- } \\
\text { tion (selection bias) }\end{array}$ & Low risk & Computer-generated randomisation list \\
\hline $\begin{array}{l}\text { Allocation concealment } \\
\text { (selection bias) }\end{array}$ & Unclear risk & $\begin{array}{l}\text { Allocation completed by independent statistician; not clear if randomisation } \\
\text { list concealed }\end{array}$ \\
\hline $\begin{array}{l}\text { Blinding of participants } \\
\text { and personnel (perfor- } \\
\text { mance bias) } \\
\text { All outcomes }\end{array}$ & High risk & Blinding not possible due to the nature of the intervention \\
\hline $\begin{array}{l}\text { Blinding of outcome as- } \\
\text { sessment (detection bias) } \\
\text { All outcomes }\end{array}$ & High risk & Self-reported \\
\hline $\begin{array}{l}\text { Selective reporting (re- } \\
\text { porting bias) }\end{array}$ & Low risk & Primary outcome measure prespecified in protocol paper and reported \\
\hline Other bias & Low risk & Contamination not described and is unlikely \\
\hline Recruitment to cluster & Low risk & $\begin{array}{l}\text { All grade } 6 \text { students from consenting schools were invited to be assessed for } \\
\text { eligibility }\end{array}$ \\
\hline Baseline imbalances & Low risk & Baseline imbalances accounted for in analysis \\
\hline Loss of clusters & Low risk & No report of loss of entire clusters \\
\hline Incorrect analysis & Low risk & Analysis adjusted for clustering \\
\hline $\begin{array}{l}\text { Compatability with indi- } \\
\text { vidually randomised trials } \\
\text { (herd effect) }\end{array}$ & Unclear risk & Unable to determine if herd effect exists \\
\hline
\end{tabular}

Mason 2012

\begin{tabular}{ll} 
Methods & Design: RCT \\
& Follow-up: $4 \& 9$ mo (post randomisation) \\
& Attrition: $3.3 \%(4 \mathrm{mo}), 6.7 \%(9 \mathrm{mo})$ \\
& Unit of randomisation: family \\
\hline Participants & N of subjects randomised: \\
& Int: 16 \\
& Ctrl: 14 \\
& Total $\mathrm{N}: 30$ \\
& N of subjects at 9 mo: \\
& Int: 16 \\
Ctrl: 12 \\
Total N: 28 \\
Age: mean: $13.9 ;$ range 12 to $15 \mathrm{yrs}$ \\
Sex (male): $56.5 \%$ \\
Ethnicity: slightly more than $50 \%$ Caucasian, $20 \%$ Hispanic, other unspecified
\end{tabular}


Mason 2012 (Continued)

Country: USA

Interventions Number of experimental conditions: 2

Description of Intervention: Project Hope programme was created by combining 2 interventions - 1 for depression and 1 for substance abuse. Parents, children, and families are provided with educational material and psychological counselling over 10 sessions, and further follow-up material (4 booklets via mail) and forum discussion ( 2 weeks later)

Type of intervention: selective (children of depressed parents)

Focus/target: prevention of both depression and substance abuse among adolescent-aged children

Fidelity: across all sessions, > 90\% key components were implemented

Dose: of the 16 randomised intervention families, 13 completed all sessions and 2 received only a partial dose of the intervention (1 session or 4 sessions)

Duration/frequency: 4 mo, 6 sessions with parents and youth; 3 sessions for parents alone; 1 session with youth alone; 4 phone contacts

Control: no programme

Length of follow-up: $4 \& 9$ mo (post randomisation)
Alcohol outcomes:
Quantity - number of drinks; adolescents were asked typical quantity of alcohol consumption on a
scale 0 "I don't drink alcohol" to 5 "More than 6 drinks"

Funding and Declared Funded by grant \#5R21DA023687-02 from the National Institute on Drug Abuse (NIDA). Study authors Conflicts of Interest declared no conflicts of interest

Notes

\section{Risk of bias}

\begin{tabular}{lll}
\hline Bias & Authors' judgement & Support for judgement \\
\hline $\begin{array}{l}\text { Random sequence genera- } \\
\text { tion (selection bias) }\end{array}$ & Unclear risk & Not described \\
\hline $\begin{array}{l}\text { Allocation concealment } \\
\text { (selection bias) }\end{array}$ & Unclear risk & Not described \\
\hline
\end{tabular}

Blinding of participants High risk Blinding not possible due to the nature of the intervention
and personnel (perfor-
mance bias)
All outcomes

Blinding of outcome as- High risk Self-reported
sessment (detection bias) All outcomes

\begin{tabular}{|c|c|c|}
\hline $\begin{array}{l}\text { Incomplete outcome data } \\
\text { (attrition bias) } \\
\text { All outcomes }\end{array}$ & Low risk & $\begin{array}{l}\text { Attrition low, attrition too small }(n=1 \text { and } n=2) \text { to look at differences between } \\
\text { groups, imputation not described, intention-to-treat analysis cited }\end{array}$ \\
\hline
\end{tabular}

Selective reporting (re- Unclear risk $\quad$ No protocol or clinical trial registry available
porting bias)


Mason 2012 (Continued)

\begin{tabular}{|c|c|c|}
\hline Other bias & Low risk & Contamination not described and is unlikely \\
\hline Recruitment to cluster & Unclear risk & Not applicable \\
\hline Baseline imbalances & Unclear risk & Not applicable \\
\hline Loss of clusters & Unclear risk & Not applicable \\
\hline Incorrect analysis & Unclear risk & Not applicable \\
\hline $\begin{array}{l}\text { Compatability with indi- } \\
\text { vidually randomised trials } \\
\text { (herd effect) }\end{array}$ & Unclear risk & Not applicable \\
\hline
\end{tabular}

Milburn 2012

\begin{tabular}{ll} 
Mesign: $\mathrm{RCT}$ \\
Follow-up: $3,6, \& 12 \mathrm{mo}$ (post randomisation) \\
Attrition: $29 \%(3 \mathrm{mo}), 42 \%(6 \mathrm{mo}), 54 \%(12 \mathrm{mo})$ \\
Unit of randomisation: family \\
\hline N of subjects randomised: \\
Int: 68 \\
Ctrl: 83 \\
Total N: 151 \\
N of subjects at 12 mo: \\
\\
Int: 68 \\
Ctrl: 83 \\
Total N: 151 \\
Age: mean: 14.8 (SD = 1.4); range 12 to 17 yrs \\
Sex (male): $33.8 \%$ \\
Ethnicity: $61.6 \%$ Hispanic, $11.3 \%$ White, $20.5 \%$ African American, $6.6 \%$ other \\
Country: USA
\end{tabular}

Interventions

\section{Number of experimental conditions: 2}

Description of Intervention: Support To Reunite, Involve and Value Each Other (STRIVE) - consisted of 5 sessions administered to youth and parent(s) together by a trained facilitator at a site selected by the family (usually home). Session content was based on CBT theories, designed to improve problem-solving and conflict resolution skills. The conceptual frame underlying the intervention highlights the importance of establishing a positive family climate, improving family functioning by routinely resolving conflicts in a mutually beneficial manner, learning how to recognise and effectively manage feelings, increasing positive affirmations, learning and practising problem-solving skills, and providing role clarity. Sessions are based on a set of very highly interactive, semi-structured tasks involving repeated feedback and practice. New skills are learned based on preceding sessions, and the skills learned in 1 session are constantly reinforced in subsequent sessions. The intervention includes the following tools: tokens to strengthen desired behaviours, a feeling thermometer to teach emotional regulation, a "thinkfeel-do" problem-solving model to operationalise and tackle problems, role-playing for safely practising new skills, and reframing to conceptualise problems and solutions in a non-blaming manner

Type of intervention: selective (families with a homeless adolescent)

Focus/target: to reduce risk taking (sexual risk behaviour, drug use) and delinquent behaviours among newly homeless youth in Southern California

Fidelity: overall $98 \%$ of tasks were implemented with fidelity 
Milburn 2012 (Continued)

Dose: attendance was high: $76 \%$ of families completed all 5 sessions, $6 \%$ attended 3 to 4 sessions, $16 \%$ attended 1 to 2 sessions, and only 1 family did not attend any sessions

Duration/frequency: 5 sessions once per week for 1.5 to $2 \mathrm{hrs}$

Control: standard care that they were receiving from the agencies that referred them. If not actively receiving any services, families were given appropriate referrals based on their needs

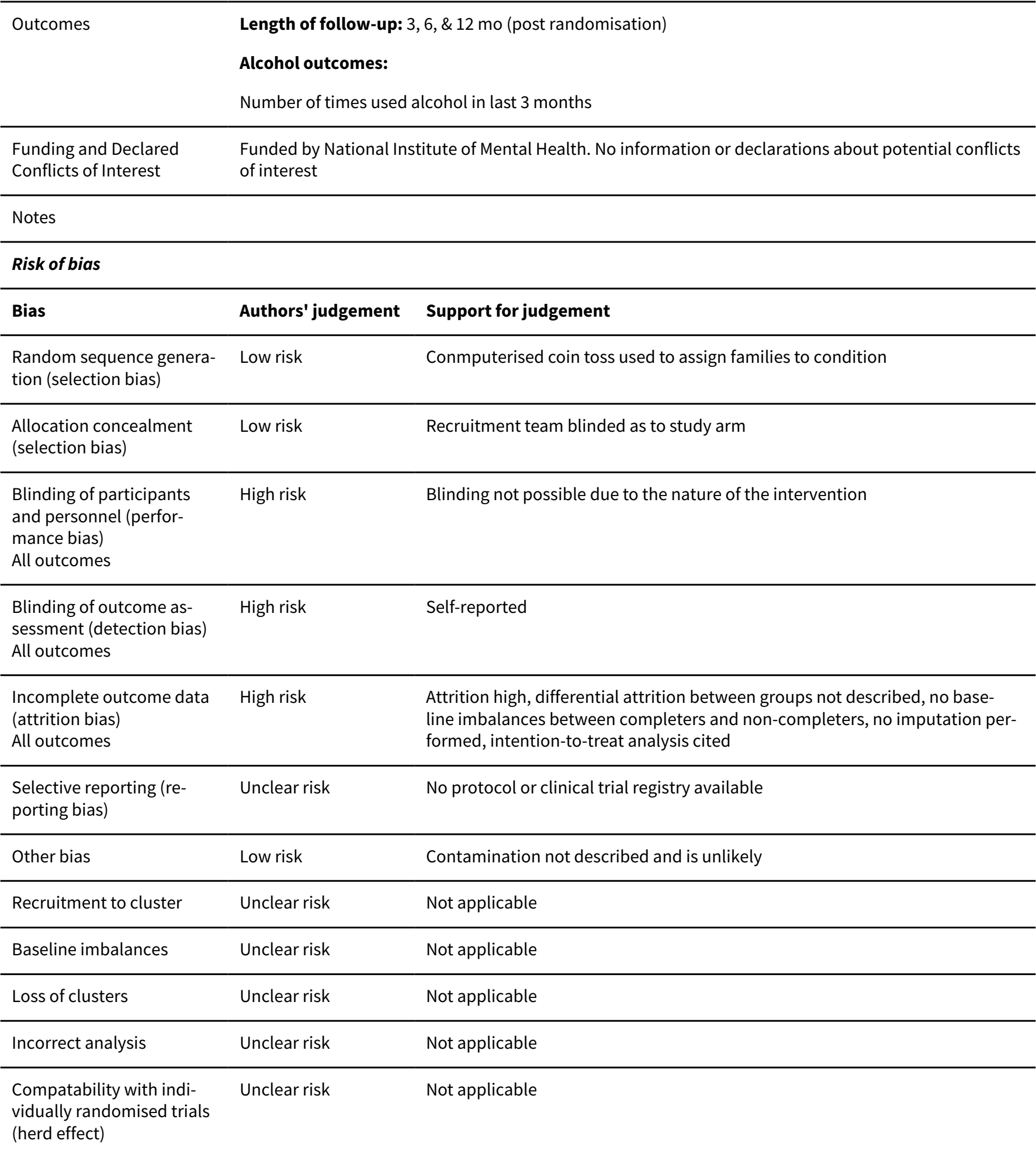




\begin{tabular}{ll}
\hline Design: RCT & Follow-up: 3 mo (post intervention) \\
& Attrition: $17 \%(3 \mathrm{mo})$ \\
& Unit of randomisation: family \\
\hline Participants & N of subjects randomised: \\
& Int: NR \\
& Ctrl: NR \\
& Total N: 268 girls \\
& N of subjects at 3 mo: \\
& Int: NR \\
Ctrl: NR & Total N: 222 \\
& Age: mean NR; range 11 to 13 yrs \\
Sex (male): $0 \%$ (all girls) & Ethnicity: $34.3 \%$ Latina \\
Country: USA
\end{tabular}

Interventions

Number of experimental conditions: 3

Description of Intervention 1: especially for daughters, a gender-specific culturally relevant parent education programme, based on social development model, delivered through a set of 4 audio CDs. CDs contain stories of lives of 4 fictional families (Latino and African American); each story aims to increase parents' awareness of the risks their daughters may face

Description of Intervention 2: an attention controlled condition; 4-booklet set of visually appealing print materials covering similar topics and mailed at the same intervals as the CDs

Type of intervention: universal

Focus/target: delaying sex and alcohol use

Fidelity: NR

Dose: about $90 \%$ of parents remembered they had gotten CDs in the mail, $93 \%$ of parents had listened to $\mathrm{CDs},>80 \%$ reported having listened with the child participant; all parents requested the Especially for Daughters CDs at the end of the study

Duration/frequency: 24 wks (each CD or booklet mailed every 6 wks)

Control: no programme

Outcomes Length of follow-up: 3 mo (post randomisation)

Alcohol outcomes:

In past year had more than a few sips of alcohol or had been drunk (yes/no)

Parents asked if they had communicated about reasons for not drinking, how to say no to drinking with friends, how drinking is not a solution to problems, how drinking does not make a girl more grown up (yes/no) 
O'Donnell 2010 (Continued)

\section{Risk of bias}

\begin{tabular}{|c|c|c|}
\hline Bias & Authors' judgement & Support for judgement \\
\hline $\begin{array}{l}\text { Random sequence genera- } \\
\text { tion (selection bias) }\end{array}$ & Unclear risk & Not described \\
\hline $\begin{array}{l}\text { Allocation concealment } \\
\text { (selection bias) }\end{array}$ & Unclear risk & Not described \\
\hline $\begin{array}{l}\text { Blinding of participants } \\
\text { and personnel (perfor- } \\
\text { mance bias) } \\
\text { All outcomes }\end{array}$ & High risk & Blinding not possible due to the nature of the intervention \\
\hline $\begin{array}{l}\text { Blinding of outcome as- } \\
\text { sessment (detection bias) } \\
\text { All outcomes }\end{array}$ & High risk & Self-reported \\
\hline $\begin{array}{l}\text { Incomplete outcome data } \\
\text { (attrition bias) } \\
\text { All outcomes }\end{array}$ & Low risk & $\begin{array}{l}\text { Attrition low, no differential attrition between groups, imputation performed, } \\
\text { intention-to-treat analysis cited }\end{array}$ \\
\hline $\begin{array}{l}\text { Selective reporting (re- } \\
\text { porting bias) }\end{array}$ & Unclear risk & No protocol or clinical trial registry available \\
\hline Other bias & Low risk & Contamination not described and is unlikely \\
\hline Recruitment to cluster & Unclear risk & Not applicable \\
\hline Baseline imbalances & Unclear risk & Not applicable \\
\hline Loss of clusters & Unclear risk & Not applicable \\
\hline Incorrect analysis & Unclear risk & Not applicable \\
\hline $\begin{array}{l}\text { Compatability with indi- } \\
\text { vidually randomised trials } \\
\text { (herd effect) }\end{array}$ & Unclear risk & Not applicable \\
\hline
\end{tabular}

Perry 2003

\begin{tabular}{ll}
\hline Methods & Design: cluster RCT \\
& Follow-up: $18 \mathrm{mo}$ (post randomisation) \\
& Attrition: $16 \%$ (18 mo) \\
Unit of randomisation: school \\
Clustering effect adjusted: yes
\end{tabular}

Participants

\section{$\mathbf{N}$ of clusters (subjects) randomised:}

Int 1: 8 (2226)

Int 2: 8 (2221)

Ctrl: 8 (1790)

Total N: 24 (6237) 
Perry 2003 (Continued)

\section{$\mathrm{N}$ of clusters (subjects) at $18 \mathrm{mo}$ :}

Int 1: $8(2518)$

Int 2: 8 (2635)

Ctrl: 8 (2108)

Total N: 24 (7261)

Age: mean: NR; range NR (7th to 8th grade)

Sex (male): $51.6 \%$

Ethnicity: $67.3 \%$ White, 7.5\% African American, 12.7\% Asian American, 3.6\% Hispanic, 4\% American Indian, $4.9 \%$ mixed/other racial groups

Country: USA

Interventions

\section{Number of experimental conditions: 3}

Description of Intervention 1: Drug Abuse Resistance Education (D.A.R.E.) included a 10-session curriculum providing skills in resisting influence to use drugs and in handling violent situations. Also focused on character building and citizenship skills

Description of Intervention 2: D.A.R.E. Plus included the D.A.R.E. component as described for Intervention 1. Additional components included a classroom-based, peer-led, parental involvement programme called VERGE (teen magazine with activities focusing on influences and skills related to peers, social groups, media, and role models) led by trained peer leaders. The magazine also included activities for students to complete with parents at home. Students participated in a theatre production in their classrooms and received 3 postcards in the mail. As a follow-up to VERGE, 10 additional postcards were mailed to parents every 6 to 8 weeks with short behavioural messages. The second component involved extracurricular activities for students (developed by Youth Action Teams). 8 community advisors were hired to create and facilitate the programmes. The final component involved neighbourhood action teams

Type of intervention: universal

Focus/target: reduce tobacco, alcohol and marijuana use, and violent behaviour

Fidelity: NR

Dose: NR

Duration/Frequency: classroom component once a week for 4 weeks, parent postcards sent over the school year

Control: no programme

Lutcomes
Alcohol outcomes:
Past year alcohol use - number of occasions
Past month alcohol use - number of occasions
Drunk - number of occasions

Funding and Declared Conflicts of Interest
Funded by Grant from the National Institute on Drug Abuse. No information or declarations about potential conflicts of interest

Notes

Participant data reported as per published paper - baseline survey completion lower than 18-month follow-up numbers

\section{Risk of bias}

Bias Authors' judgement Support for judgement


Perry 2003 (Continued)

\begin{tabular}{|c|c|c|}
\hline $\begin{array}{l}\text { Random sequence genera- } \\
\text { tion (selection bias) }\end{array}$ & Unclear risk & Not described \\
\hline $\begin{array}{l}\text { Allocation concealment } \\
\text { (selection bias) }\end{array}$ & Unclear risk & Not described \\
\hline $\begin{array}{l}\text { Blinding of participants } \\
\text { and personnel (perfor- } \\
\text { mance bias) } \\
\text { All outcomes }\end{array}$ & High risk & Blinding not possible due to the nature of the intervention \\
\hline $\begin{array}{l}\text { Blinding of outcome as- } \\
\text { sessment (detection bias) } \\
\text { All outcomes }\end{array}$ & High risk & Self-reported \\
\hline $\begin{array}{l}\text { Incomplete outcome data } \\
\text { (attrition bias) } \\
\text { All outcomes }\end{array}$ & Low risk & $\begin{array}{l}\text { Attrition low, no differential attrition between groups, regarding the analytical } \\
\text { approach - "...one of the strengths of this approach is its ability to appropriate- } \\
\text { ly model missing data" }\end{array}$ \\
\hline $\begin{array}{l}\text { Selective reporting (re- } \\
\text { porting bias) }\end{array}$ & Unclear risk & No protocol of clinical trial registry available \\
\hline Other bias & Low risk & Contamination not described and is unlikely \\
\hline Recruitment to cluster & Unclear risk & $\begin{array}{l}\text { Unclear as to whether recruitment occurred before or after randomisation of } \\
\text { clusters }\end{array}$ \\
\hline Baseline imbalances & Low risk & Matching of schools based on socioeconomic measures, drug use, and size \\
\hline Loss of clusters & Low risk & No report of loss of entire clusters \\
\hline Incorrect analysis & Low risk & Analysis adjusted for clustering \\
\hline $\begin{array}{l}\text { Compatability with indi- } \\
\text { vidually randomised trials } \\
\text { (herd effect) }\end{array}$ & Unclear risk & Unable to determine if herd effect exists \\
\hline
\end{tabular}

Prado 2012

Methods

Design: RCT

Follow-up: $6 \& 12 \mathrm{mo}$ (post randomisation)

Attrition: $7 \%$ (6 mo), 9\% (12 mo)

Unit of randomisation: family

Participants N of subjects randomised:

Int: 120

Ctrl: 122

Total N: 242

$\mathrm{N}$ of subjects at $12 \mathrm{mo}$ :

Int: 113

Ctrl: 116

Total N: 229

Age: mean: $14.7(\mathrm{SD}=1.38)$; range 12 to $17 \mathrm{yrs}$

Sex (male): $65 \%$ 
Prado 2012 (Continued)

Ethnicity: 100\% Hispanic, $65 \%$ born in USA; immigrant youth (84) and parents were primarily born in Cuba (25\%), Honduras (15.5\%), Nicaragua (9.5\%), Puerto Rico (8.3\%), and Dominican Republic (7.1\%) Country: USA

Interventions
Number of experimental conditions: 2
Description of Intervention: eight $2 \mathrm{hr}$ parent group sessions aimed to bring parents together for the
purposes of establishing parental investment, increasing parental support, and providing a context for
parent participation in a conjoint skills learning process. Four $1 \mathrm{hr}$ family visits aimed to provide par-
ents with an opportunity to transfer the competencies learned in the group sessions to their adoles-
cent, foster more nurturing and supportive relationships, and increase parent-child communication -
all in the context of family. Adolescents participated only in the family visits
Type of intervention: selective (delinquent youth)
Focus/target: prevent substance use and unsafe sexual behaviour
Fidelity: NR
Dose: the actual mean number of sessions attended was 6.88 (SD $=4.05)$; among those who attended
at least 1 session ( $87 \%)$, the mean number of sessions was 7.9 (SD $=3.2)$
Duration/frequency: 12 wks, $8 \times 2$ hrs multi-parent group sessions, and $4 \times 1$ hr family visits
Control: standard care services were offered (referrals to community-based organisations that offer
several therapeutic modalities, including individual and family therapy, and address multiple problem
behaviours, including alcohol and drug use)

Outcomes

Length of follow-up: 6 \& 12 mo (post randomisation)

\section{Alcohol outcomes:}

Alcohol consumption last 90 days (yes/no)

Alcohol dependence diagnosis (diagnostic interview schedule for children)

Funding and Declared Conflicts of Interest
Funded by National Institute on Drug Abuse Grant. No information or declarations about potential conflicts of interest

\section{Notes}

\section{Risk of bias}

\begin{tabular}{lll}
\hline Bias & Authors' judgement & Support for judgement \\
\hline $\begin{array}{l}\text { Random sequence genera- } \\
\text { tion (selection bias) }\end{array}$ & Unclear risk & Not described \\
\hline $\begin{array}{l}\text { Allocation concealment } \\
\text { (selection bias) }\end{array}$ & Low risk & $\begin{array}{l}\text { The allocator was not aware of the condition assignment before participant } \\
\text { enrolment }\end{array}$ \\
\hline $\begin{array}{l}\text { Blinding of participants } \\
\text { and personnel (perfor- } \\
\text { mance bias) }\end{array}$ & High risk & Blinding not possible due to the nature of the intervention \\
All outcomes & & \\
\hline
\end{tabular}

\begin{tabular}{ll}
\hline Blinding of outcome as- & High risk \\
sessment (detection bias) & \\
All outcomes & \\
\hline
\end{tabular}

\begin{tabular}{|c|c|c|}
\hline $\begin{array}{l}\text { Incomplete outcome data } \\
\text { (attrition bias) }\end{array}$ & Low risk & $\begin{array}{l}\text { Attrition low, no differential attrition by groups or characteristics; growth } \\
\text { curve modelling used, allowing for missing data and using all available data }\end{array}$ \\
\hline
\end{tabular}


Prado 2012 (Continued)

\begin{tabular}{|c|c|c|}
\hline $\begin{array}{l}\text { Selective reporting (re- } \\
\text { porting bias) }\end{array}$ & Unclear risk & No protocol or clinical trial registry available \\
\hline Other bias & Low risk & Contamination not described and is unlikely \\
\hline Recruitment to cluster & Unclear risk & Not applicable \\
\hline Baseline imbalances & Unclear risk & Not applicable \\
\hline Loss of clusters & Unclear risk & Not applicable \\
\hline Incorrect analysis & Unclear risk & Not applicable \\
\hline $\begin{array}{l}\text { Compatability with indi- } \\
\text { vidually randomised trials } \\
\text { (herd effect) }\end{array}$ & Unclear risk & Not applicable \\
\hline
\end{tabular}

Reddy 2002

Mesign: cluster RCT
Follow-up: $12 \mathrm{mo}$ (post randomisation)
Attrition: $6.8 \%(12 \mathrm{mo})$
Unit of randomisation: school
Clustering effect adjusted: yes
N of clusters (subjects) randomised:
Int 1: 10 (NR)
Int 2: 10 (NR)
Ctrl: 10 (NR)
Total N: 30 (5043)
N of clusters (subjects) at 12 mo:
Int $1: 10(1769)$
Int 2: $10(1293)$
Ctrl: $10(1390)$
Total N: $30(4452)$
Age: mean: $11.9 ;$ range 11 to 13 yrs (7th grade)
Sex (male): $50.5 \%$
Ethnicity: NR
Country: India

Interventions

Number of experimental conditions: 3

Description of Intervention 1: Health-Related Information and Dissemination Among Youth (HRIDAY) + family component. HRIDAY consisted of (1) a set of 10 posters promoting cardiovascular health, (2) copies of the HRIDAY booklet for each classroom, (3) classroom activities selected by teachers from a training manual of 20 activities ranging from 30 to 60 mins, (4) debates within and between schools on banning tobacco sponsorship and follow-up focus group discussions, and (5) roundtable discussions on nutrition and food policy. The family-based programme involved students taking home 6 booklets and activities to share with their families. Teacher training workshops were held. Student peer leaders from each school (2) were trained in leading workshops

Description of Intervention 2: HRIDAY component as described for Intervention 1 
Type of intervention: universal

Focus/target: to improve cardiovascular health among young adolescents, including dietary change, physical activity, and smoking prevention. Alcohol use was included but was not the focus of the programme

Fidelity: For Int 1 and Int 2 combined: 14 schools displayed all 10 HRIDAY posters, whereas the other 6 displayed 7 to 9 posters; 9 schools circulated the booklet among students; 10 schools implemented activities from the teachers' manual, of which 6 schools implemented all 20 activities; all intervention schools participated in the signature campaign; 16 schools held debates within their schools on banning tobacco sponsorship; and 16 schools also participated in debates with other schools. The degree of implementation did not vary between Int 1 and Int 2; for the 10 schools in Int 1 (with the family component), teachers in 8 schools distributed at least 5 of 6 booklets

Dose: NR

Duration/frequency: $9 \mathrm{mo}$

Control: no programme

\begin{tabular}{ll} 
Outcomes & $\begin{array}{l}\text { Length of follow-up: } 12 \text { mo (post randomisation) } \\
\text { Alcohol outcomes: } \\
\text { Have you ever had a drink of alcohol? (yes/no) }\end{array}$ \\
\hline $\begin{array}{l}\text { Funding and Declared } \\
\text { Conflicts of Interest }\end{array}$ & $\begin{array}{l}\text { Project HRIDAY was funded by the Fogarty International Research Collaboration Award (RO3-TW00729), } \\
\text { National Institutes of Health, Washington, DC. No information or declarations about potential conflicts } \\
\text { of interest }\end{array}$
\end{tabular}

\section{Notes}

\section{Risk of bias}

\begin{tabular}{lll}
\hline Bias & Authors' judgement & Support for judgement \\
\hline $\begin{array}{l}\text { Random sequence genera- } \\
\text { tion (selection bias) }\end{array}$ & Unclear risk & Not described \\
\hline $\begin{array}{l}\text { Allocation concealment } \\
\text { (selection bias) }\end{array}$ & Unclear risk & Not described \\
\hline $\begin{array}{l}\text { Blinding of participants } \\
\text { and personnel (perfor- } \\
\text { mance bias) } \\
\text { All outcomes }\end{array}$ & High risk & Blinding not possible due to the nature of the intervention \\
\hline
\end{tabular}

\begin{tabular}{lll}
\hline $\begin{array}{l}\text { Blinding of outcome as- } \\
\text { sessment (detection bias) } \\
\text { All outcomes }\end{array}$ & High risk & Self-reported \\
\hline $\begin{array}{l}\text { Incomplete outcome data } \\
\text { (attrition bias) } \\
\text { All outcomes }\end{array}$ & Low risk & $\begin{array}{l}\text { Attrition low, no differential attrition between groups, method for dealing with } \\
\text { missing data not described }\end{array}$ \\
\hline $\begin{array}{l}\text { Selective reporting (re- } \\
\text { porting bias) }\end{array}$ & Unclear risk & No protocol or clinical trial registry available \\
\hline Other bias & Low risk & $\begin{array}{l}\text { Study authors note that schools did not share educational materials, so conta- } \\
\text { mination is unlikely }\end{array}$ \\
\hline
\end{tabular}


Reddy 2002 (Continued)

\begin{tabular}{|c|c|c|}
\hline Recruitment to cluster & Unclear risk & $\begin{array}{l}\text { Unclear as to whether recruitment occurred before or after randomisation of } \\
\text { clusters }\end{array}$ \\
\hline Baseline imbalances & Low risk & $\begin{array}{l}\text { Stratification of clusters based on school type (public/private) and gender } \\
\text { makeup }\end{array}$ \\
\hline Loss of clusters & Low risk & No report of loss of entire clusters \\
\hline Incorrect analysis & Low risk & Analysis adjusted for clustering \\
\hline $\begin{array}{l}\text { Compatability with indi- } \\
\text { vidually randomised trials } \\
\text { (herd effect) }\end{array}$ & Unclear risk & Unable to determine if herd effect exists \\
\hline
\end{tabular}

\section{Riesch 2012}

$\begin{array}{ll}\text { Methods } & \text { Design: cluster RCT } \\ & \text { Follow-up: immediate post-test, } 6 \text { mo (post randomisation) } \\ & \text { Attrition: } 34 \% \text { (from randomisation to } 6 \text { mo) } \\ & \text { Unit of randomisation: school }\end{array}$

Clustering effect adjusted: yes

Participants N of clusters (subjects) randomised:

Int: 9 (105)

Ctrl: 7 (83)

Total N: 16 (188)

$\mathrm{N}$ of clusters (subjects) at 6 mo:

Int: 9 (66)

Ctrl: 7 (59)

Total N: 16 (125)

Age: mean: $10.8(\mathrm{SD}=0.7)$; range 9 to $11 \mathrm{yrs}$

Sex (male): $50 \%$ to $51 \%$

Ethnicity: $>50 \%$ of adult-youth dyads were African American

Country: USA

\section{Number of experimental conditions: 2}

Description of Intervention: Strengthening Families Program 10-14 (SFP10-14) - youth and 1 parent attended the 7-week, 2-hr-per-week programme together at community locations in the evenings or weekends in each city. Didactic content was presented by videotape, discussion sessions were timed, and the curriculum was detailed carefully in a manual that contained all the required handouts. Youth and parents or legal guardians were separated for the first of the 2 consecutive hours to work on goaloriented, developmentally appropriate activities. The dyads were reunited in the second hour for family-oriented activities. The sessions were characterised by lively activities and interactions

Type of intervention: universal

Focus/target: preventing substance use

Fidelity: filled-in checklists, not clear by whom; more than $90 \%$ of the content was consistently covered in parent groups and $87 \%$ in youth groups

Dose: NR

Duration/frequency: $7 \times 2 \mathrm{hr}$ weekly sessions

Control: no programme 
Riesch 2012 (Continued)

Outcomes

Length of follow-up: immediate post-test, 6 mo (post randomisation)

\section{Alcohol outcomes:}

If they had drunk alcohol without their parents' permission (yes/no)

Funding and Declared Conflicts of Interest
The work was supported by the Substance Abuse and Mental Health Services Administration. Study authors declared no conflicts of interest

\section{Notes}

\section{Risk of bias}

\begin{tabular}{|c|c|c|}
\hline Bias & Authors' judgement & Support for judgement \\
\hline $\begin{array}{l}\text { Random sequence genera- } \\
\text { tion (selection bias) }\end{array}$ & Unclear risk & Not described \\
\hline $\begin{array}{l}\text { Allocation concealment } \\
\text { (selection bias) }\end{array}$ & Unclear risk & Not described \\
\hline $\begin{array}{l}\text { Blinding of participants } \\
\text { and personnel (perfor- } \\
\text { mance bias) } \\
\text { All outcomes }\end{array}$ & High risk & Blinding not possible due to the nature of the intervention \\
\hline $\begin{array}{l}\text { Blinding of outcome as- } \\
\text { sessment (detection bias) } \\
\text { All outcomes }\end{array}$ & High risk & Self-reported \\
\hline $\begin{array}{l}\text { Incomplete outcome data } \\
\text { (attrition bias) } \\
\text { All outcomes }\end{array}$ & High risk & $\begin{array}{l}\text { Attirition high, differential attrition between groups apparent but significance } \\
\text { not clear, no differential attrition between completers and non-completers, } \\
\text { imputation not described, intention-to-treat analysis cited }\end{array}$ \\
\hline $\begin{array}{l}\text { Selective reporting (re- } \\
\text { porting bias) }\end{array}$ & Unclear risk & No protocol or clinical trial registry available \\
\hline Other bias & Low risk & Contamination not described and is unlikely \\
\hline Recruitment to cluster & High risk & $\begin{array}{l}\text { Recruitment occurred after randomisation of schools, so possibility for influ- } \\
\text { ence on the type of participants recruited }\end{array}$ \\
\hline Baseline imbalances & Low risk & Stratification of clusters based on proportion of minority group involvement \\
\hline Loss of clusters & Low risk & No report of loss of entire clusters \\
\hline Incorrect analysis & Low risk & Analysis adjusted for clustering \\
\hline $\begin{array}{l}\text { Compatability with indi- } \\
\text { vidually randomised trials } \\
\text { (herd effect) }\end{array}$ & Unclear risk & Unable to determine if herd effect exists \\
\hline
\end{tabular}

Schinke 2004

$\begin{array}{ll}\text { Methods } & \text { Design: cluster RCT } \\ & \text { Follow-up: immediate post-test, 1, 2, 3, 6, \& } 7 \text { yrs (post randomisation) }\end{array}$


Schinke 2004 (Continued)

Attrition: Int $17.9 \%$, Int $211.8 \%$, Ctrl 6.7\% (3 yrs), 20\% overall (6 yrs), Int $123.9 \%$, Int $221.6 \%$, Ctrl

$14.7 \%$ ( 7 yrs)

Unit of randomisation: community

Clustering effect adjusted: no

\begin{tabular}{|c|c|}
\hline Participants & $\begin{array}{l}\text { N of subjects randomised: } \\
\text { Int 1: NR } \\
\text { Int 2: NR } \\
\text { Ctrl: NR } \\
\text { Total N: } 514 \\
\text { N of subjects at } 3 \text { yrs: } \\
\text { Int 1: NR } \\
\text { Int 2: NR } \\
\text { Ctrl: NR } \\
\text { Total N: } 469 \\
\text { Age: mean: } 11.5 \text { (SD = } 0.53) \text {; range } 10 \text { to } 12 \text { yrs } \\
\text { Sex (male): } 48.6 \% \\
\text { Ethnicity: } 54 \% \text { Black, } 30 \% \text { Hispanic, } 11 \% \text { were White, } 5 \% \text { from other ethnic-racial groups } \\
\text { Country: USA }\end{array}$ \\
\hline
\end{tabular}

Interventions

Number of experimental conditions: 3

Description of Intervention 1: CD-ROM + Parent programme (CDP). The CD component consisted of $10 \times 45$ minute sessions for the youth incorporating social learning and problem behaviour theories (goal-setting, coping, peer pressure, refusal skills, norm-correcting, self-efficacy, problem-solving, decision-making, effective communication, and time management). Sessions were completed at home/ in the community. Youths applied problem-solving to work through the interactive animated sessions. Youths received booster sessions between follow-up measurements (annually). For the parent component, parents received an initial in-home intervention via printed materials and videotape. Parents were taught how to enhance the youth intervention via discussion between parents and youths of skills that youths were learning, how to help youths apply programmatic content, and how to support youths when they avoided substance use and engaged in health-promoting activities. Booster sessions for parents were delivered mostly by CD-ROM and digital audio recordings, the latter sessions via an iPod. Parents were also engaged in a workshop to help them better understand and manage their children

Description of Intervention 2: CD-ROM programme (CD) as described for CD component of Intervention 1 including youth booster sessions

Type of intervention: universal

Focus/target: alcohol and other substance use prevention Fidelity: NR

Dose: CD-ROM intervention \& CD-ROM plus parent intervention, $95 \%$ and $91 \%$ of youths, respectively, completed both initial and booster interventions; for parents, 163 (83\%) watched the videotape, 131 (67\%) attended the workshop, and 155 (79\%) completed the interactive CD-ROM exercise with their adolescent children

Duration/frequency: CD component of Int 1 and Int $2-10 \times 45$ minute sessions, $5 \times$ youth booster sessions; parent component of Int 1 - initial sessions included 30 min videotape, $2 \times$ parent booster sessions

Control: no programme

\section{Alcohol outcomes:}

30 day alcohol use - number of times 
Schinke 2004 (Continued)

How many times in the past month have your parents talked to you about not drinking alcohol?

Length of follow-up: 6 \& 7 yrs (post randomisation)

\section{Alcohol outcomes:}

30 day alcohol use - number of times

Length of follow-up: 7 yrs (post randomisation)

Alcohol outcomes:

$\geq 5$ drinks in a row - number of occasions

Funding and Declared Conflicts of Interest
Funding received through the National Institute on Alcohol Abuse and Alcoholism (grant AA11924). No information or declarations about potential conflicts of interest

\section{Notes}

\section{Risk of bias}

\begin{tabular}{|c|c|c|}
\hline Bias & Authors' judgement & Support for judgement \\
\hline $\begin{array}{l}\text { Random sequence genera- } \\
\text { tion (selection bias) }\end{array}$ & Unclear risk & Not described \\
\hline $\begin{array}{l}\text { Allocation concealment } \\
\text { (selection bias) }\end{array}$ & Unclear risk & Not described \\
\hline $\begin{array}{l}\text { Blinding of participants } \\
\text { and personnel (perfor- } \\
\text { mance bias) } \\
\text { All outcomes }\end{array}$ & High risk & $\begin{array}{l}\text { Blinding of participants and personnel not possible due to the nature of the in- } \\
\text { tervention. }\end{array}$ \\
\hline $\begin{array}{l}\text { Blinding of outcome as- } \\
\text { sessment (detection bias) } \\
\text { All outcomes }\end{array}$ & High risk & $\begin{array}{l}\text { Self-reported; blinding of participants and personnel not possible due to the } \\
\text { nature of the intervention }\end{array}$ \\
\hline $\begin{array}{l}\text { Incomplete outcome data } \\
\text { (attrition bias) } \\
\text { All outcomes }\end{array}$ & Unclear risk & $\begin{array}{l}\text { Attrition low, differential attrition by group not described, no differential attri- } \\
\text { tion by characteristics }\end{array}$ \\
\hline $\begin{array}{l}\text { Selective reporting (re- } \\
\text { porting bias) }\end{array}$ & Unclear risk & No protocol or clinical trial registry available \\
\hline Other bias & Low risk & Contamination not described and is unlikely \\
\hline Recruitment to cluster & Low risk & Clusters randomised after participant recruitment \\
\hline Baseline imbalances & Low risk & $\begin{array}{l}\text { No baseline imbalances; clusters were stratified: "...collaborating sites were } \\
\text { stratified by geography and ethnic-racial background of the youth population } \\
\text { served" }\end{array}$ \\
\hline Loss of clusters & Low risk & No report of loss of entire clusters \\
\hline Incorrect analysis & Low risk & $\begin{array}{l}\text { Analysis not sufficiently adjusted for clustering; review authors performed an } \\
\text { alternative adjustment }\end{array}$ \\
\hline
\end{tabular}


Schinke 2004 (Continued)
Compatability with indi-
Unclear risk
Unable to determine if herd effect exists

vidually randomised trials

(herd effect)

\section{Schinke 2009a}

\begin{tabular}{ll} 
Methods & Design: RCT \\
& Follow-up: 3 wks (post randomisation) or immediately post-test, 11 wks (post randomisation) or 2 mo \\
(post intervention) & Attrition: $1 \%$ to $2 \%(2$ mo) \\
& Unit of randomisation: mother-daughter dyad \\
\hline Participants & N of subjects randomised: \\
& Int: NR \\
& Ctrl: NR \\
& Total N: 202 \\
& N of subjects at 2 mo: \\
& Int: NR \\
Ctrl: NR & Total N: NR \\
Age: mean: 12.2 (SD $=0.95)$ \\
Sex (male): $0 \%$ (girls only) \\
Ethnicity: $67.8 \%$ White, 14.1\% Latina, 9.5\% Black, $0.5 \%$ Asian, $8 \%$ Other \\
Country: USA
\end{tabular}

Interventions

\section{Number of experimental conditions: 2}

Description of Intervention: computer-mediated gender-specific programme based on family interaction theory aims to (1) enhance quality of daughters' relationship with their mothers, and (2) teach girls cognitive-behavioural skills. Exercises taught girls and mothers about value of listening to each other, spending time together, negotiating during arguments, giving compliments, and providing personal favours. Completion by mothers - 14 computer-mediated intervention modules; modules 1 to 5 (rapport and respect building between daughters and mothers), modules 6 to 10 (conflict management, ground rules for negotiating arguments, empathic listening), and modules 11 to 14 (help participants analyse media portrayal of drinking)

Type of intervention: universal

Focus/target: alcohol use

Fidelity: participants could advance to the next session only if each separately answered correctly questions on the prior session

Dose: NR

Duration/frequency: 3 wks

Control: no programme

\section{Alcohol outcomes:}

30-day alcohol use - number of alcohol drinks consumed

7-day alcohol use - number of alcohol drinks consumed

1-year alcohol use - number of alcohol drinks consumed 
Schinke 2009a (Continued)

Funding and Declared Conflicts of Interest
This research was supported by National Institute on Drug Abuse grant DA17721. No information or declarations about potential conflicts of interest

Notes

\section{Risk of bias}

\begin{tabular}{|c|c|c|}
\hline Bias & Authors' judgement & Support for judgement \\
\hline $\begin{array}{l}\text { Random sequence genera- } \\
\text { tion (selection bias) }\end{array}$ & Unclear risk & Not described \\
\hline $\begin{array}{l}\text { Allocation concealment } \\
\text { (selection bias) }\end{array}$ & Unclear risk & Not described \\
\hline $\begin{array}{l}\text { Blinding of participants } \\
\text { and personnel (perfor- } \\
\text { mance bias) } \\
\text { All outcomes }\end{array}$ & High risk & Blinding not possible due to the nature of the intervention \\
\hline $\begin{array}{l}\text { Blinding of outcome as- } \\
\text { sessment (detection bias) } \\
\text { All outcomes }\end{array}$ & High risk & Self-reported \\
\hline $\begin{array}{l}\text { Incomplete outcome data } \\
\text { (attrition bias) } \\
\text { All outcomes }\end{array}$ & Low risk & $\begin{array}{l}\text { Attrition low, attrition too low to detect differences between groups, imputa- } \\
\text { tion not described }\end{array}$ \\
\hline $\begin{array}{l}\text { Selective reporting (re- } \\
\text { porting bias) }\end{array}$ & Unclear risk & No protocol or clinical trial registry available \\
\hline Other bias & Low risk & Contamination not described and is unlikely \\
\hline Recruitment to cluster & Unclear risk & Not applicable \\
\hline Baseline imbalances & Unclear risk & Not applicable \\
\hline Loss of clusters & Unclear risk & Not applicable \\
\hline Incorrect analysis & Unclear risk & Not applicable \\
\hline $\begin{array}{l}\text { Compatability with indi- } \\
\text { vidually randomised trials } \\
\text { (herd effect) }\end{array}$ & Unclear risk & Not applicable \\
\hline
\end{tabular}

Schinke 2009b

\begin{tabular}{ll}
\hline Methods & Design: RCT \\
& Follow-up: $1 \& 2 \mathrm{yrs}$ (post randomisation) \\
& $\begin{array}{l}\text { Attrition: } 5.7 \%(1 \mathrm{yr}), 9 \%(2 \mathrm{yrs}) \\
\text { Unit of randomisation: mother-daughter dyad }\end{array}$ \\
\hline Participants & N of subjects randomised: \\
& Int: 458 \\
Ctrl: 458
\end{tabular}


Schinke 2009b (Continued)

Total N: 916

$\mathrm{N}$ of subjects at $2 \mathrm{yrs}$ :

Int: 415

Ctrl: 413

Total N: 828

Age: mean: $12.76(\mathrm{SD}=1.0)$

Sex (male): $0 \%$ (girls only)

Ethnicity: $23.2 \%$ White, 23.1\% Latina, 40.6\% Black, 10.8\% Asian, 1.7\% Other

Country: USA

Interventions

\section{Number of experimental conditions: 2}

Description of Intervention: computer-mediated gender-specific programme based on family interaction theory. Mothers learnt to better communicate with their daughters, monitor their daughters' activities, build their daughters' self-image and self-esteem, establish rules and consequences for substance abuse, create family rituals, and refrain from placing unrealistic expectations on their daughters. Girls learnt to manage stress and conflict/mood, refuse peer pressure, and enhance their body esteem. Programme exercises taught girls and mothers about value of listening to each other, spending time together, negotiating during arguments, giving compliments, and providing personal favours

Type of intervention: universal

Focus/target: substance use

Fidelity: participants could advance to the next session only if each separately answered correctly questions on the prior session

Dose: participants could not access post-intervention and follow-up measures unless they finished all programme sessions

Duration/frequency: 9 wks ( 9 sessions 45 min each per week)

Control: no programme

\section{Outcomes}

Length of follow-up: 3 \& 11 wks (post randomisation)

\section{Alcohol outcomes:}

30-day alcohol use - number of occasions

Funding and Declared

No information or declarations about funding or potential conflicts of interest.

Conflicts of Interest

Notes

\section{Risk of bias}

\begin{tabular}{lll}
\hline Bias & Authors' judgement & Support for judgement \\
\hline $\begin{array}{l}\text { Random sequence genera- } \\
\text { tion (selection bias) }\end{array}$ & Unclear risk & Not described \\
\hline $\begin{array}{l}\text { Allocation concealment } \\
\text { (selection bias) }\end{array}$ & Unclear risk & Not described \\
\hline $\begin{array}{l}\text { Blinding of participants } \\
\text { and personnel (perfor- } \\
\text { mance bias) }\end{array}$ & High risk & Blinding not possible due to the nature of the intervention \\
All outcomes & \\
\hline
\end{tabular}

Blinding of outcome as- High risk Self-reported

sessment (detection bias) 
Schinke 2009b (Continued)

All outcomes

\begin{tabular}{|c|c|c|}
\hline $\begin{array}{l}\text { Incomplete outcome data } \\
\text { (attrition bias) } \\
\text { All outcomes }\end{array}$ & Low risk & $\begin{array}{l}\text { Attrition low, no differential attrition between groups, no differential attrition } \\
\text { by characteristics, imputation not described }\end{array}$ \\
\hline $\begin{array}{l}\text { Selective reporting (re- } \\
\text { porting bias) }\end{array}$ & Unclear risk & No protocol or clinical trial registry available \\
\hline Other bias & Low risk & Contamination not described and is unlikely \\
\hline Recruitment to cluster & Unclear risk & Not applicable \\
\hline Baseline imbalances & Unclear risk & Not applicable \\
\hline Loss of clusters & Unclear risk & Not applicable \\
\hline Incorrect analysis & Unclear risk & Not applicable \\
\hline $\begin{array}{l}\text { Compatability with indi- } \\
\text { vidually randomised trials } \\
\text { (herd effect) }\end{array}$ & Unclear risk & Not applicable \\
\hline
\end{tabular}

\section{Schinke 2009c}

\begin{tabular}{ll}
\hline Methods & Design: $\mathrm{RCT}$ \\
& Follow-up: immediate post-test, $1 \mathrm{yr}$ (post intervention) \\
& Attrition: $1.5 \%$ (post-test), $10 \%(1 \mathrm{yr})$ \\
& Unit of randomisation: mother-daughter dyad \\
\hline
\end{tabular}

Participants $\quad$ N of subjects randomised:

Int: 252

Ctrl: 339

Total N: 591

$\mathbf{N}$ of subjects at $1 \mathrm{yr}$ :

Int: 205

Ctrl: 327

Total N: 532

Age: mean: $12.7(\mathrm{SD}=1.0)$

Sex (male): $0 \%$ (girls only)

Ethnicity: Int vs Ctrl: $39 \%$ vs $17 \%$ White; $22 \%$ vs $26 \%$ Latina; $39 \%$ vs $56 \%$ Black

Country: USA

\section{Interventions $\quad$ Number of experimental conditions: 2}

Description of Intervention: computer-mediated gender-specific programme based on family interaction theory. Mothers learnt to better communicate with their daughters, monitor their daughters' activities, build their daughters' self-image and self-esteem, establish rules and consequences for substance abuse, create family rituals, and refrain from placing unrealistic expectations on their daughters. Girls learnt to manage stress and conflict/mood. Through animated vignettes and video demonstrations, girls and mothers learnt how depression can result from stress and pressure to succeed. Session interactive activity showed the importance of valuing personal character and accomplishments. Programme aim was to enhance emotional closeness between girls and mothers

Type of intervention: universal 
Schinke 2009c (Continued)

Focus/target: substance use

Fidelity: participants could advance to the next session only if each separately answered correctly questions on the prior session

Dose: participants could not access post-intervention and follow-up measures unless they finished all programme sessions

Duration/frequency: 9 wks (9 sessions 45 min each per week)

Control: no programme

Outcomes Length of follow-up: immediate post-test, $1 \mathrm{yr}$ (post intervention)

\section{Alcohol outcomes:}

30 day use of alcohol - number of drinks consumed

Funding and Declared Conflicts of Interest
No information or declarations about funding or potential conflicts of interest

\section{Notes}

\section{Risk of bias}

\begin{tabular}{|c|c|c|}
\hline Bias & Authors' judgement & Support for judgement \\
\hline $\begin{array}{l}\text { Random sequence genera- } \\
\text { tion (selection bias) }\end{array}$ & Unclear risk & Not described \\
\hline $\begin{array}{l}\text { Allocation concealment } \\
\text { (selection bias) }\end{array}$ & Unclear risk & Not described \\
\hline $\begin{array}{l}\text { Blinding of participants } \\
\text { and personnel (perfor- } \\
\text { mance bias) } \\
\text { All outcomes }\end{array}$ & High risk & Blinding not possible due to the nature of the intervention \\
\hline $\begin{array}{l}\text { Blinding of outcome as- } \\
\text { sessment (detection bias) } \\
\text { All outcomes }\end{array}$ & High risk & Self-reported \\
\hline $\begin{array}{l}\text { Incomplete outcome data } \\
\text { (attrition bias) } \\
\text { All outcomes }\end{array}$ & Unclear risk & $\begin{array}{l}\text { Attrition low, differential attrition by group not described, no differential attri- } \\
\text { tion by characteristics, imputation not described }\end{array}$ \\
\hline $\begin{array}{l}\text { Selective reporting (re- } \\
\text { porting bias) }\end{array}$ & Unclear risk & No protocol or clinical trial registry available \\
\hline Other bias & Low risk & Contamination not described and is unlikely \\
\hline Recruitment to cluster & Unclear risk & Not applicable \\
\hline Baseline imbalances & Unclear risk & Not applicable \\
\hline Loss of clusters & Unclear risk & Not applicable \\
\hline Incorrect analysis & Unclear risk & Not applicable \\
\hline $\begin{array}{l}\text { Compatability with indi- } \\
\text { vidually randomised trials } \\
\text { (herd effect) }\end{array}$ & Unclear risk & Not applicable \\
\hline
\end{tabular}


Schinke 2011

$\begin{array}{ll}\text { Methods } & \text { Design: RCT } \\ & \text { Follow-up: immediate post-test } \\ & \text { Attrition: } 4.6 \% \text { (post-test) } \\ & \text { Unit of randomisation: parent-adolescent dyad } \\ & \text { N of subjects randomised: } \\ & \text { Int: } 212 \\ \text { Ctrl: } 334 & \\ \text { Total N: } 546 \\ \text { N of subjects at post-test: } \\ \text { Int: } 198 \\ \text { Ctrl: } 323 \\ \text { Total N: } 521 \\ \text { Age: } \text { mean: } 12.75 \text { (SD = 1.02); range } 10 \text { to } 13 \text { yrs } \\ \text { Sex (male): } 0 \% \text { (girls only) } \\ \text { Ethnicity: } 65.2 \% \text { Black, } 34.1 \% \text { Hispanic } \\ \text { Country: USA }\end{array}$

Interventions

\section{Number of experimental conditions: 2}

Description of Intervention: CD-ROM/Internet programme for mothers and daughters that included 10 CD-ROM or Internet sessions that could be completed at home at a convenient time. The programme aimed to reduce substance use through mother-daughter interactions and communication. Targeted mother-daughter communication, behaviour, and activities; daughters' self-image and selfesteem; rules about and consequences for substance use; family rituals; and refraining from communicating unrealistic expectations. Girls acquired skills for managing stress, conflict, mood, and anxiety; refusing peer pressure; and enhancing body esteem and self-efficacy. Girls also learned accurate information about the prevalence of smoking, drinking, and drug use among their peers. The programme included interactive components (animated characters, interactive exercises, problem-solving)

Type of intervention: universal

Focus/target: substance use

Fidelity: participants could advance to the next session only after completing the previous one

Dose: NR

Duration/frequency: 10 sessions, advised to complete 1 per week

Control: no programme

Outcomes Length of follow-up: immediate post-test

\section{Alcohol outcomes:}

30-day alcohol use - number of alcohol drinks consumed

\section{Risk of bias}

\begin{tabular}{lll}
\hline Bias & Authors' judgement & Support for judgement \\
\hline $\begin{array}{l}\text { Random sequence genera- } \\
\text { tion (selection bias) }\end{array}$ & Unclear risk & Not described \\
\hline
\end{tabular}


Schinke 2011 (Continued)

\begin{tabular}{|c|c|c|}
\hline $\begin{array}{l}\text { Allocation concealment } \\
\text { (selection bias) }\end{array}$ & Unclear risk & Not described \\
\hline $\begin{array}{l}\text { Blinding of participants } \\
\text { and personnel (perfor- } \\
\text { mance bias) } \\
\text { All outcomes }\end{array}$ & High risk & Blinding not possible due to the nature of the intervention \\
\hline $\begin{array}{l}\text { Blinding of outcome as- } \\
\text { sessment (detection bias) } \\
\text { All outcomes }\end{array}$ & High risk & Self-reported \\
\hline $\begin{array}{l}\text { Incomplete outcome data } \\
\text { (attrition bias) } \\
\text { All outcomes }\end{array}$ & Unclear risk & Attrition low, differential attrition not described \\
\hline $\begin{array}{l}\text { Selective reporting (re- } \\
\text { porting bias) }\end{array}$ & Unclear risk & No protocol or clinical trial registry available \\
\hline Other bias & Low risk & Contamination not described and is unlikely \\
\hline Recruitment to cluster & Unclear risk & Not applicable \\
\hline Baseline imbalances & Unclear risk & Not applicable \\
\hline Loss of clusters & Unclear risk & Not applicable \\
\hline Incorrect analysis & Unclear risk & Not applicable \\
\hline $\begin{array}{l}\text { Compatability with indi- } \\
\text { vidually randomised trials } \\
\text { (herd effect) }\end{array}$ & Unclear risk & Not applicable \\
\hline
\end{tabular}

Skarstrand 2014

$\begin{array}{ll}\text { Methods } & \text { Design: cluster RCT } \\ \text { Follow-up: } 1,2, \& 3 \text { yrs (post randomisation) } \\ \text { Attrition: } 24 \%(3 \text { yrs) } \\ \text { Unit of randomisation: school } \\ \text { Clustering effect adjusted: yes }\end{array}$

Participants

$\mathrm{N}$ of clusters (subjects) randomised:

Int: $10(441)$

Ctrl: 9 (266)

Total N: 22 (707)

$\mathrm{N}$ of clusters (subjects) at 3 yrs:

Int: $10(283)$

Ctrl: 9 (164)

Total N: 19 (447)

Age: mean: 12 (6th grade)

Sex (male): $49.7 \%$

Ethnicity: NR 
Skarstrand 2014 (Continued)

Country: Sweden
Interventions Number of experimental conditions: 2

Description of Intervention: cultural adaptation of the SFP 10-14 to Swedish conditions. Included youth and parent separate concurrent sessions and family sessions. Youth sessions included role-playing, peer resistance training, and practical skill development. Parent sessions included video contents illustrating typical parent-youth interactions. Family sessions included family projects. Part 1 included $7 \times 1 \mathrm{hr}$ sessions ( 6 separate sessions for youth and parents, and 1 joint family session), and part 2 included $5 \times 1 \mathrm{hr}$ sessions ( 4 separate sessions for youth and parents, and 1 joint family session)

Type of intervention: universal

Focus/target: substance use

Fidelity: checklists for intervention content, which group leaders filled out after each session, \% NR

Dose: all youth received training, and participation of parents was voluntary (47\% of youths were represented by at least 1 parent in part 1 of the programme, and $27 \%$ in part 2)

Duration/frequency: 7 consecutive wks in grade 6 ( $1 \mathrm{hr}$ per week), 5 consecutive wks in grade 7 ( $1 \mathrm{hr}$ per week)

Control: no programme
Funding and Declared Conflicts of Interest

\section{Length of follow-up: $1,2, \& 3$ yrs (post randomisation)}

\section{Alcohol outcomes:}

Lifetime drunkenness

Drunkenness last 30 days - number of occasions

Drunkenness last 30 days - numbers of times dichotomised to 'any drunkenness' versus 'no drunkenness'

Funding provided by the Swedish Council for Working Life and Social Research. Study authors declared no conflicts of interest

\section{Notes}

\section{Risk of bias}

\begin{tabular}{lll}
\hline Bias & Authors' judgement & Support for judgement \\
\hline $\begin{array}{l}\text { Random sequence genera- } \\
\text { tion (selection bias) }\end{array}$ & Unclear risk & Not described \\
\hline $\begin{array}{l}\text { Allocation concealment } \\
\text { (selection bias) }\end{array}$ & Unclear risk & Not described \\
\hline $\begin{array}{l}\text { Blinding of participants } \\
\text { and personnel (perfor- } \\
\text { mance bias) } \\
\text { All outcomes }\end{array}$ & High risk & Blinding not possible due to the nature of the intervention \\
\hline $\begin{array}{l}\text { Blinding of outcome as- } \\
\text { sessment (detection bias) } \\
\text { All outcomes }\end{array}$ & High risk & Self-reported \\
\hline $\begin{array}{l}\text { Incomplete outcome data } \\
\text { (attrition bias) } \\
\text { All outcomes }\end{array}$ & High risk & Attrition high, no differential attrition between groups, multiple imputation \\
\hline
\end{tabular}


Skarstrand 2014 (Continued)

Selective reporting (re- Unclear risk No protocol or clinical trial registry available porting bias)

Other bias High risk

Study authors noted that control schools had other alcohol intervention(s) occurring at the time of the intervention

Recruitment to cluster High risk

After randomisation, 5 schools wanted to include more than 1 class per school in the study. It may have been possible that participants in classes allocated to the intervention group were more motivated/engaged as schools requested more classes after allocation to the intervention group

\begin{tabular}{lll}
\hline Baseline imbalances & Low risk & Baseline imbalances accounted for in the analysis \\
\hline Loss of clusters & Low risk & No report of loss of entire clusters \\
\hline Incorrect analysis & Low risk & Analysis adjusted for clustering \\
\hline $\begin{array}{l}\text { Compatability with indi- } \\
\begin{array}{l}\text { vidually randomised trials } \\
\text { (herd effect) }\end{array}\end{array}$ & Unclear risk & Unable to determine if herd effect exists \\
\hline
\end{tabular}

Spirito 2011

\begin{tabular}{ll}
\hline Methods & Design: RCT \\
& Follow-up: $3,6, \& 12 \mathrm{mo}$ (post randomisation) \\
& Attrition: $22 \%(3 \mathrm{mo}), 26 \%(6 \mathrm{mo}), 34 \%(12 \mathrm{mo})$ \\
& Unit of randomisation: family \\
\hline Participants & N of subjects randomised: \\
& Int $1: 63$ \\
& Int 2: 62 \\
& Total $\mathrm{N}: 125$ \\
& N of subjects at 12 mo: \\
& Int 1: 47 \\
Int 2: 36 & Total N: 83 \\
Age: mean: 15 (SD $=1.2) ;$ range 13 to 17 yrs \\
Sex (male): Int $147.6 \%$, Int $245.2 \%$ \\
Ethnicity: Int 1 vs Int 2: $71.4 \%$ vs $61.3 \%$ Non-Hispanic White, $27 \%$ vs. $27.4 \%$ Hispanic \\
Country: USA
\end{tabular}

Description of Intervention 1: Individual Motivational Interview (IMI) and Family Check-Up (FCU). IMI included a 45 minute counselling session focusing on personal responsibility, exploration of motivation for drinking, and review of potential negative consequences; personalised normative feedback; establishing goals regarding drinking; and anticipating barriers to accomplishing goals. FCU was a $1 \mathrm{hr}$ videotaped family assessment task that included discussing family beliefs regarding alcohol and drug use, as well as other topics (e.g. curfew). One week after the assessment task, parents received feedback. Potential barriers to change were discussed

Description of Intervention 2: Individual Motivational Interview (IMI) as described for Intervention 1

Type of intervention: indicated (youth treated in an emergency department after an alcohol-related event and with a positive blood alcohol concentration) 
Spirito 2011 (Continued)

Focus/target: reduce alcohol use

Fidelity: IMI: independent raters coded the sessions, averaged $83 \%$ for fidelity to intervention protocol; FCU: fidelity to the components of the FCU ranged from $79 \%$ to $100 \%$

Dose: NR

Duration/frequency: Int $2-1 \times 45$ minute session

Control: not applicable

Outcomes

Length of follow-up: 3,6 , \& 12 mo (post randomisation)

\section{Alcohol outcomes:}

Number of drinking days per month

Quantity - drinks per occasion

$\geq 5$ drink days per month

$\geq 5$ drinks per occasion in previous 3 months (yes/no)

Funding and Declared Conflicts of Interest
Funding from the National Institute on Alcohol Abuse and Alcoholism. The funder had no role in study design; collection, analysis, and interpretation of data; writing of the report; and the decision to submit the paper for publication. Study authors declared no conflicts of interest

Notes

\section{Risk of bias}

\begin{tabular}{|c|c|c|}
\hline Bias & Authors' judgement & Support for judgement \\
\hline $\begin{array}{l}\text { Random sequence genera- } \\
\text { tion (selection bias) }\end{array}$ & Unclear risk & Not described \\
\hline $\begin{array}{l}\text { Allocation concealment } \\
\text { (selection bias) }\end{array}$ & Low risk & $\begin{array}{l}\text { Randomly assigned to the IMI or the IMI+FCU condition by interventionists } \\
\text { based on condition assignments contained in sealed envelopes }\end{array}$ \\
\hline $\begin{array}{l}\text { Blinding of participants } \\
\text { and personnel (perfor- } \\
\text { mance bias) } \\
\text { All outcomes }\end{array}$ & High risk & $\begin{array}{l}\text { Blinding of participants and personnel not possible due to the nature of the in- } \\
\text { tervention }\end{array}$ \\
\hline $\begin{array}{l}\text { Blinding of outcome as- } \\
\text { sessment (detection bias) } \\
\text { All outcomes }\end{array}$ & High risk & $\begin{array}{l}\text { Self-reported; blinding of participants and personnel not possible due to the } \\
\text { nature of the intervention }\end{array}$ \\
\hline $\begin{array}{l}\text { Incomplete outcome data } \\
\text { (attrition bias) } \\
\text { All outcomes }\end{array}$ & High risk & Attrition high, no differential attrition between groups \\
\hline $\begin{array}{l}\text { Selective reporting (re- } \\
\text { porting bias) }\end{array}$ & Low risk & $\begin{array}{l}\text { Reported outcomes consistent with the clinical trial registry: clinicaltrials.gov } \\
\text { identifier NCT00247221 }\end{array}$ \\
\hline Other bias & Low risk & Contamination not described and is unlikely \\
\hline Recruitment to cluster & Unclear risk & Not applicable \\
\hline Baseline imbalances & Unclear risk & Not applicable \\
\hline Loss of clusters & Unclear risk & Not applicable \\
\hline
\end{tabular}


Spirito 2011 (Continued)

\begin{tabular}{lll} 
Incorrect analysis & Unclear risk & Not applicable \\
\hline $\begin{array}{l}\text { Compatability with indi- } \\
\text { vidually randomised trials } \\
\text { (herd effect) }\end{array}$ & Unclear risk & Not applicable \\
\hline
\end{tabular}

Spirito 2015

$\begin{array}{ll}\text { Methods } & \text { Design: } \mathrm{RCT} \\ & \text { Follow-up: } 6 \text { mo (post randomisation) } \\ & \text { Attrition: } 12 \%(6 \mathrm{mo}) \\ & \text { Unit of randomisation: family }\end{array}$

Participants N of subjects randomised:

Int 1: 32

Int 2: 35

Total N: 67

$\mathrm{N}$ of subjects at $6 \mathrm{mo}$ :

Int 1: 26

Int 2: 33

Total N: 59

Age: mean: NR; range 11 to $17 \mathrm{yrs}$

Sex (male): Int $156.3 \%$, Int $254.3 \%$

Ethnicity: predominantly White, Non-Hispanic, and Hispanic/Latino

Country: USA

Interventions

\section{Number of experimental conditions: 2}

Description of Intervention 1: Family Check-Up (FCU) involved assessment and feedback, based on motivational interviewing principles and designed to enhance parental recognition of child risk behaviours and to engender motivation for reducing these problem behaviours and associated risk factors. The intervention targets specific family risk and protective factors linked to alcohol and drug use, including parental supervision and monitoring and parent-child relationship quality. Both interventions received 8 'booster' brochures (mailed out)

Description of Intervention 2: Psychoeducation session (PE) - attended a baseline assessment and 1 alcohol and drug use educational session. PE parents were also provided with a number of handouts about abuse, dependence, and high-risk situations. Both interventions received 8 'booster' brochures (mailed out)

Type of intervention: selective (youth receiving services for an emotional or behavioural disorder)

Focus/target: delay or prevent the onset of alcohol and drug use

Fidelity: adherence to protocol components was $87 \%$

Dose: FCU - all but 1 finished the intervention in its entirety

Duration/frequency: both interventions $-1 \times 60$ to 90 minute session and 8 booster mail-out brochures at 3 week intervals between baseline and follow-up

Control: not applicable

Length of follow-up: 6 mo (post randomisation)

\section{Alcohol outcomes:}

Composite from the Youth Alcohol and Drug Survey: had alcohol in past 3 mo, how long had they been using alcohol, whether they had at least 1 drink in the past 7 days and the past 30 days, total number 
Spirito 2015 (Continued)

of drinks consumed in past 7 days and past 30 days, whether they had consumed 5 or more drinks in a row in the past 2 weeks and in the past 30 days

Alcohol and Drug Communication Scale, alcohol subscale

Funding and Declared Conflicts of Interest
Funded by National Institute on Drug Abuse Grant. No information or declarations about potential conflicts of interest

\section{Notes}

\section{Risk of bias}

\begin{tabular}{|c|c|c|}
\hline Bias & Authors' judgement & Support for judgement \\
\hline $\begin{array}{l}\text { Random sequence genera- } \\
\text { tion (selection bias) }\end{array}$ & Low risk & Urn randomisation procedure \\
\hline $\begin{array}{l}\text { Allocation concealment } \\
\text { (selection bias) }\end{array}$ & Unclear risk & Not described \\
\hline $\begin{array}{l}\text { Blinding of participants } \\
\text { and personnel (perfor- } \\
\text { mance bias) } \\
\text { All outcomes }\end{array}$ & High risk & $\begin{array}{l}\text { Blinding of participants and personnel not possible due to the nature of the in- } \\
\text { tervention }\end{array}$ \\
\hline $\begin{array}{l}\text { Blinding of outcome as- } \\
\text { sessment (detection bias) } \\
\text { All outcomes }\end{array}$ & High risk & $\begin{array}{l}\text { Self-reported; blinding of participants and personnel not possible due to the } \\
\text { nature of the intervention }\end{array}$ \\
\hline $\begin{array}{l}\text { Incomplete outcome data } \\
\text { (attrition bias) } \\
\text { All outcomes }\end{array}$ & Unclear risk & Attrition low, differential attrition apparent but significance unclear \\
\hline $\begin{array}{l}\text { Selective reporting (re- } \\
\text { porting bias) }\end{array}$ & Unclear risk & No protocol or clinical trial registry available \\
\hline Other bias & Low risk & Contamination not described and is unlikely \\
\hline Recruitment to cluster & Unclear risk & Not applicable \\
\hline Baseline imbalances & Unclear risk & Not applicable \\
\hline Loss of clusters & Unclear risk & Not applicable \\
\hline Incorrect analysis & Unclear risk & Not applicable \\
\hline $\begin{array}{l}\text { Compatability with indi- } \\
\text { vidually randomised trials } \\
\text { (herd effect) }\end{array}$ & Unclear risk & Not applicable \\
\hline
\end{tabular}

Spirito 2017

$\begin{array}{ll}\text { Methods } & \text { Design: } \mathrm{RCT} \\ & \text { Follow-up: } 3 \mathrm{mo}, 6 \mathrm{mo}, 1 \mathrm{yr} \text { (post randomisation) } \\ & \text { Attrition: } 16.7 \%(1 \mathrm{yr})\end{array}$


Spirito 2017 (Continued)

Unit of randomisation: family

Participants $\quad$ N of subjects randomised:

Int 1: 51

Int 2: 51

Total N: 102

$\mathrm{N}$ of subjects at $12 \mathrm{mo}$ :

Int 1: 44

Int 2: 41

Total N: 85

Age: mean: Int $115.85(S D=1.3)$, Int $215.93(S D=1.37)$; range 12 to 19 yrs

Sex (male): Int $153.2 \%$, Int $266.7 \%$

Ethnicity: Int 1 vs Int 2: $44.7 \%$ vs $35.6 \%$ White, $27.7 \%$ vs $31.1 \%$ Hispanic, $2.1 \%$ vs $15.6 \%$ Black, $2.1 \%$ vs $0 \%$ Native American, $2.1 \%$ vs $2.2 \%$ Asian

Country: USA

Number of experimental conditions: 2

Description of Intervention 1: Family Check-Up (FCU) involved assessment and feedback, based on motivational interviewing principles and designed to educate parents about risk for AOD use among adolescents, support appropriate parenting, and motivate parents to change ineffective parenting. If asked, counsellors also provided advice on how to change parenting behaviour

Description of Intervention 2: Psychoeducation (PE) participants attended a baseline assessment and 1 alcohol and drug use educational session. The session ended with handouts provided about the topics reviewed in the session. In both FCU and PE conditions, parents were mailed 8 booster brochures every 3 to 4 weeks over the 6 -month follow-up period

Type of intervention: indicated (adolescents who were using alcohol or marijuana and whose parents were concerned about their use)

Focus/target: reduce alcohol and drug use among adolescents already using and their close in age siblings

Fidelity: on average, $84 \%$ of expected components of the FCU were administered. On average, $97 \%$ of expected elements of the PE session were delivered by the counsellors

Dose: NR

Duration/frequency: both interventions $-1 \times 60$ to 90 minute session and 8 booster mail-out brochures at 3 week intervals between baseline and follow-up

Control: not applicable

Outcomes

Length of follow-up: $3 \mathrm{mo}, 6 \mathrm{mo}, 1 \mathrm{yr}$ (post randomisation)

Alcohol outcomes: measured using the Adolescent Drinking Questionnaire

How often was alcohol consumed in the last 3 months?

Number of drinks in last month

Heavy drinking assessed with question "In the last 3 months, how many times did you had 5 or more drinks when you were drinking?" Responses recorded as number of drinking days and number of heavy drinking days

Funding and Declared Conflicts of Interest
Funded by National Institute on Drug Abuse and Alcoholism Grant. No information or declarations about potential conflicts of interest

\section{Notes \\ $\mathrm{RCT}=$ randomised controlled trial; $\mathrm{N}=$ number; Int=intervention; $\mathrm{Ctrl}=$ control; $\mathrm{mo}=$ month(s);} $\mathrm{yr}(\mathrm{s})=\mathrm{year}(\mathrm{s}) ; \mathrm{NR}=$ not reported.

\section{Risk of bias}


Spirito 2017 (Continued)

\begin{tabular}{|c|c|c|}
\hline Bias & Authors' judgement & Support for judgement \\
\hline $\begin{array}{l}\text { Random sequence genera- } \\
\text { tion (selection bias) }\end{array}$ & Low risk & Urn randomisation procedure \\
\hline $\begin{array}{l}\text { Allocation concealment } \\
\text { (selection bias) }\end{array}$ & Low risk & Opaque envelopes used \\
\hline $\begin{array}{l}\text { Blinding of participants } \\
\text { and personnel (perfor- } \\
\text { mance bias) } \\
\text { All outcomes }\end{array}$ & High risk & $\begin{array}{l}\text { Blinding of participants and personnel not possible due to the nature of the in- } \\
\text { tervention }\end{array}$ \\
\hline $\begin{array}{l}\text { Blinding of outcome as- } \\
\text { sessment (detection bias) } \\
\text { All outcomes }\end{array}$ & High risk & $\begin{array}{l}\text { Most outcomes self-reported; blinding of participants and personnel not possi- } \\
\text { ble due to the nature of the intervention }\end{array}$ \\
\hline $\begin{array}{l}\text { Incomplete outcome data } \\
\text { (attrition bias) } \\
\text { All outcomes }\end{array}$ & Unclear risk & $\begin{array}{l}\text { Attrition low, differential attrition between groups apparent but significance } \\
\text { not clear, no differential attrition by characteristics }\end{array}$ \\
\hline $\begin{array}{l}\text { Selective reporting (re- } \\
\text { porting bias) }\end{array}$ & Unclear risk & No protocol or clinical trial registry available \\
\hline Other bias & Low risk & Contamination not described and is unlikely \\
\hline Recruitment to cluster & Unclear risk & Not applicable \\
\hline Baseline imbalances & Unclear risk & Not applicable \\
\hline Loss of clusters & Unclear risk & Not applicable \\
\hline Incorrect analysis & Unclear risk & Not applicable \\
\hline $\begin{array}{l}\text { Compatability with indi- } \\
\text { vidually randomised trials } \\
\text { (herd effect) }\end{array}$ & Unclear risk & Not applicable \\
\hline
\end{tabular}

\section{Spoth 1999a}

Design: cluster RCT
Follow-up: $18,30,48,72, \& 120$ mo (post randomisation)
Attrition: $32 \%$ to $36 \%$ across different follow-up points
Unit of randomisation: school

Clustering effect adjusted: yes

Participants N of clusters (subjects) randomised:

Int 1: $11(437)$

Int 2: $11(463)$

Ctrl: 11 (409)

Total N: 33 (1309)

$\mathrm{N}$ of clusters (subjects) at $48 \mathrm{mo}$ :

Int 1: $11(152)$ 
Spoth 1999a (Continued)

Int 2: 11 (144)

Ctrl: $11(151)$

Total N: 33 (447)

Age: mean: $11.3(\mathrm{SD}=0.03)$

Sex (male): $46 \%$

Ethnicity: 99\% White

Country: USA

Description of Intervention 1: lowa Strengthening Families Program (ISFP) was based on the biopsychosocial model and other empirically based family risk and protective factor models including the resiliency and social ecology models of adolescent substance use. ISFP included weekly sessions consisting of 6 separate but concurrent parent and child curricula at 1 hour each, followed by six 1 hour joint family sessions where parents and children practice skills that they learnt; the seventh session included only a 1 hour family session. Parents are taught to clarify expectations, use appropriate disciplinary practices, manage strong emotions of children, and engage in effective communication with children; children additionally are given peer resistance and peer relationships skill training; use of videotapes

Description of Intervention 2: Preparing for Drug Free Years (PDFY) was based on the Social Development Model to enhance protective parent-child interactions and to reduce family-based risk factors for early substance use initiation. Programme goals were (1) to increase the frequency of opportunities for prosocial involvement in the family, (2) to strengthen the child's skills for prosocial involvements and resistance to antisocial influence, (3) to increase recognitions and rewards for child behaviour that conforms to family rules and expectations. Programme consisted of 5 weekly sessions, 2 hours duration each. Children attended only 1 session and parents attended all sessions. Parents were instructed on risk factors for substance use, ways to develop clear guidelines on substance-related behaviour, how to enhance parent-child bonding, how to monitor compliance with guidelines, and techniques for managing anger and family conflict. Children were instructed on peer resistance skills; use of videotapes

Type of intervention: universal

Focus/target: Int $1 \& 2$ - substance use

Fidelity: ISFP: $83 \%$ to $89 \%$ of group leaders' component tasks were covered; PDFY: $69 \%$ of group leaders' component tasks were covered

Dose: NR

Duration/frequency: Int 1 - ISFP 7 sessions in 7 wks, Int 2 - PDFY 5 sessions in 5 wks

Control: mailed 4 leaflets describing different aspects of adolescent development such as physical and emotional changes

$$
\begin{aligned}
& \text { Alcohol outcomes: } \\
& \text { \% reporting lifetime alcohol use } \\
& \% \text { reporting past month alcohol use } \\
& \text { \% reporting past year alcohol use } \\
& \% \text { reporting lifetime drunkenness } \\
& \text { Length of follow-up: } 48 \text { mo (post randomisation) } \\
& \text { Alcohol outcomes: } \\
& 48 \text { mo post randomisation } \\
& \% \text { reporting lifetime alcohol use } \\
& \% \text { reporting past month alcohol use } \\
& \text { \% reporting past year alcohol use } \\
& \% \text { reporting lifetime drunkenness } \\
& \% \text { of new alcohol users } \\
& \text { Ever drank alcohol }
\end{aligned}
$$

\section{Alcohol outcomes:}


Spoth 1999a (Continued)

Ever drunk

$\%$ of alcohol users in the past month

Past month mean frequency of drinking

Alcohol use composite index ( $0=$ no use, $1=$ use)

Length of follow-up: 72 mo (post randomisation)

\section{Alcohol outcomes:}

$\%$ reporting lifetime alcohol use

$\%$ reporting past month alcohol use

$\%$ reporting past year alcohol use

$\%$ reporting lifetime drunkenness

Alcohol composite use index ( $0=$ no use, $1=$ use)

Lifetime alcohol use

Lifetime drunkenness

Alcohol composite use index ( $0=$ no use, $1=$ use)

Lifetime alcohol use

Lifetime drunkenness

Length of follow-up: 120 mo (post randomisation)

\section{Alcohol outcomes:}

Drunkenness frequency

Alcohol problem frequency

Intervention effects on initiation growth factors

Average level of initiation drunkenness

Rate of increase in drunkenness frequency

Average level of initiation alcohol problems

Rate of increase in alcohol problem frequency

Indirect effect on drunkenness

Direct effect on drunkenness

Indirect effect on alcohol problems

Direct effect on alcohol problems

Rate of alcohol abuse

Funding and Declared

Conflicts of Interest

No information or declarations about funding or potential conflicts of interest

\section{Notes}

\section{Risk of bias}

\begin{tabular}{lll}
\hline Bias & Authors' judgement & Support for judgement \\
\hline $\begin{array}{l}\text { Random sequence genera- } \\
\text { tion (selection bias) }\end{array}$ & Unclear risk & Not described \\
\hline $\begin{array}{l}\text { Allocation concealment } \\
\text { (selection bias) }\end{array}$ & Unclear risk & Not described \\
\hline $\begin{array}{l}\text { Blinding of participants } \\
\begin{array}{l}\text { and personnel (perfor- } \\
\text { mance bias) } \\
\text { All outcomes }\end{array}\end{array}$ & High risk & Blinding not possible due to the nature of the intervention \\
\hline $\begin{array}{l}\text { Blinding of outcome as- } \\
\text { sessment (detection bias) } \\
\begin{array}{l}\text { All outcomes } \\
\hline \hline\end{array}\end{array}$ & High risk & Self-reported \\
\hline
\end{tabular}


Spoth 1999a (Continued)

Incomplete outcome data High risk Attrition high, differential attrition not described, imputation not described (attrition bias)

All outcomes

Selective reporting (re- Unclear risk $\quad$ No protocol or clinical trial registry available
porting bias)

\begin{tabular}{|c|c|c|}
\hline Other bias & Low risk & Contamination not described and is unlikely \\
\hline Recruitment to cluster & Low risk & $\begin{array}{l}\text { "Schools... then randomly assigned to the conditions. All families of sixth } \\
\text { graders in participating schools were recruited for participation. At the time } \\
\text { of pretesting families did not know the condition to which their child's school } \\
\text { had been assigned" }\end{array}$ \\
\hline Baseline imbalances & Unclear risk & $\begin{array}{l}\text { Counties matched on lunch programme eligibility and community size; other } \\
\text { baseline characteristics not described }\end{array}$ \\
\hline Loss of clusters & Low risk & No report of loss of entire clusters \\
\hline Incorrect analysis & Low risk & $\begin{array}{l}\text { Analysis not sufficiently adjusted for clustering; review authors performed an } \\
\text { alternative adjustment }\end{array}$ \\
\hline $\begin{array}{l}\text { Compatability with indi- } \\
\text { vidually randomised trials } \\
\text { (herd effect) }\end{array}$ & Unclear risk & Unable to determine if herd effect exists \\
\hline
\end{tabular}

Spoth 2002

Design: cluster RCT
Follow-up: 1 yr (post intervention), $2.5 \& 5.5$ yrs (post randomisation)
Attrition: $\%$ NR
Unit of randomisation: school
Clustering effect adjusted: yes
N of clusters (subjects) randomised:
Int 1: 12 (549)
Int 2: 12 (621)
Ctrl: 12 (494)
Total N: 36 (1664) (excluded 9 students who switched schools, 1673 originally randomised)
N of clusters (subjects) at 2.5 yrs:
Int 1: $12(399)$
Int 2: $12(369)$
Ctrl: 12 (430)
Total N: 36 (1198)
Age: mean: NR; range 10 to 14 yrs
Sex (male): $53 \%$
Ethnicity: $96 \%$ Caucasian
Country: USA

Interventions

Number of experimental conditions: 3

Description of Intervention 1: Strengthening Families Programme (SFP) for parents and youth 10 to 14 years of age and Classroom Life Skills Training (LST). SFP10-14 in the long-term targeted reduction 
of youth substance use and other problem behaviours, and in the intermediate term targeted increasing parental skills, communication, rule-setting, youth social/peer resistance skills. The 7 sessions were $2 \mathrm{hrs}$ long and included $1 \mathrm{hr}$ in which parents and youth were separate and $1 \mathrm{hr}$ in which they participated as a family. LST aimed to promote skill development, provide knowledge, and encourage avoidance of substance use. Students were trained in various LST skills through the use of interactive teaching techniques

Description of Intervention 2: LST as described for Intervention 1

Type of intervention: universal

Focus/target: substance use and other problem behaviours

Fidelity: adherence averaged at $>92 \%$. Coverage of mandatory content averaged $92 \%$ in parent, $94 \%$ in youth, and $98 \%$ in family sessions

Dose: SFP: $90 \%$ attended $>50 \%$ of sessions, SFP booster: $89 \%$ attended $>50 \%$ of sessions, LST \& LST booster: $100 \%$ attended $>50 \%$ of sessions

Duration/frequency: SFP: $7 \times 2 \mathrm{hr}$ sessions conducted once a week after school, for 7 weeks during seventh grade, 4 booster sessions in eighth grade. LST: 15 session programme during school hours of seventh grade, 5 LST booster sessions during eighth grade

Control: minimal contact with mail-outs 2 pages long summarising developmental research in lay language; addressed topics of understanding teens' emotional changes, the changing parent-child relationship, teen-related changes in thinking, and physical changes in teens or LST only

Outcomes

Length of follow-up: $1 \mathrm{yr}$ (post intervention)

\section{Alcohol outcomes:}

Proportion of new alcohol users (ever had a drink of alcohol)

Substance initiation index

Length of follow-up: $2.5 \mathrm{yr}$ (post randomisation)

\section{Alcohol outcomes:}

Substance initiation index

Regular alcohol use

Weekly drunkenness

Length of follow-up: $5.5 \mathrm{yr}$ (post randomisation)

\section{Alcohol outcomes:}

Substance initiation index

Alcohol initiation

Drunkenness initiation

Alcohol frequency for high-risk student subgroup on more serious or problematic outcomes

Drunkenness frequency for high-risk student subgroup on more serious or problematic outcomes

Length of follow-up: 8 to $10 \mathrm{yr}$ (post randomisation)

\section{Alcohol outcomes:}

Drunkenness frequency

Alcohol-related problems 
Spoth 2002 (Continued)

Funding and Declared Conflicts of Interest
Funded through the National Institute on Drug Abuse and National Institute of Mental Health. No information or declarations about potential conflicts of interest

Notes

\section{Risk of bias}

\begin{tabular}{lll}
\hline Bias & Authors' judgement & Support for judgement \\
\hline $\begin{array}{l}\text { Random sequence genera- } \\
\text { tion (selection bias) }\end{array}$ & Unclear risk & Not described \\
\hline $\begin{array}{l}\text { Allocation concealment } \\
\text { (selection bias) }\end{array}$ & Unclear risk & Not described \\
\hline $\begin{array}{l}\text { Blinding of participants } \\
\text { and personnel (perfor- } \\
\text { mance bias) }\end{array}$ & High risk & $\begin{array}{l}\text { Both schools and the experimental team were aware of the allocations. "After } \\
\text { we matched the schools and randomly assigned them to conditions, we con- } \\
\text { tacted school officials and informed them of the experimental condition to } \\
\text { which their schools had been assigned" }\end{array}$ \\
\hline $\begin{array}{l}\text { Blinding of outcome as- } \\
\text { sessment (detection bias) } \\
\text { All outcomes }\end{array}$ & High risk & $\begin{array}{l}\text { Self-reported; both schools and the experimental team were aware of the al- } \\
\text { locations. "After we matched the schools and randomly assigned them to con- } \\
\text { ditions, we contacted school officials and informed them of the experimental } \\
\text { condition to which their schools had been assigned" }\end{array}$ \\
\hline
\end{tabular}

\section{Incomplete outcome data Unclear risk Attrition not described; no differential attrition by group or characteristics} (attrition bias)

All outcomes

\begin{tabular}{lll}
\hline $\begin{array}{l}\text { Selective reporting (re- } \\
\text { porting bias) }\end{array}$ & Unclear risk & No protocol or clinical trial registry available \\
\hline Other bias & Low risk & Contamination not described and is unlikely \\
\hline Recruitment to cluster & Low risk & $\begin{array}{l}\text { All students in participating schools (already randomised) were recruited for } \\
\text { participation. Students are unlikely to change schools for the purpose of being } \\
\text { part of the intervention group }\end{array}$ \\
\hline $\begin{array}{l}\text { Baseline imbalances } \\
\text { Loss of clusters }\end{array}$ & Low risk & Baseline imbalances accounted for in the analysis \\
\hline $\begin{array}{l}\text { Incorrect analysis } \\
\begin{array}{l}\text { Compatability with indi- } \\
\text { vidually randomised trials } \\
\text { (herd effect) }\end{array}\end{array}$ & Low risk & No report of loss of entire clusters \\
\hline
\end{tabular}

Stanger 2017

Methods Design: RCT

Follow-up: 36 wks (post intervention)

Attrition: $22.7 \%$ (36 wks) 
Unit of randomisation: adolescent

Clustering effect adjusted: not applicable

Narticipants
Int: 37
Ctrl: 38
Total $\mathrm{N}: 75$
N of subjects at 36 weeks:
Int: 28
Ctrl: 30
Total $\mathrm{N}: 58$
Age: mean: 16.1 (SD $=1.2$ ); range 12 to 18 yrs
Sex (male): $75 \%$
Ethnicity: $81 \%$ Caucasian
Country: $U$ SA

Interventions Number of experimental conditions: 2

Description of Intervention: Abstinence Based Incentives (ABI) programme using clinic- and homebased ABI. Adolescents receive Motivational Enhancement Therapy (MET)/Cognitive-Behavioural Therapy (CBT); parents receive a comprehensive parent training (PT) programme based on Adolescent Transitions, an evidence-based programme targeting concerns in addition to substance use. The home-based programme instructed parents to develop a Substance Monitoring Contract (SMC) that specified weekly positive and negative consequences for abstinence or use

Type of intervention: indicated (adolescents already using alcohol or marijuana, with alcohol abuse/ dependence or a binge episode in past 90 days)

Focus/target: reduction in alcohol and cannabis use

Fidelity: Adherence to Adolescent Transitions: raters rated 2 randomly selected sessions for each Int family (95\% of families had 1 rated session). Mean overall quality score was 5.18 (SD $=1.19$ ) on a 9-point scale, indicating scores in the "acceptable" range

Adherence to MET/CBT: rated frequency/extensiveness and competence on 7 point scales; $50 \%$ of participants were randomly selected and each had $1 \mathrm{MET}$ and CBT session rated; mean frequency/extensiveness ratings were MET $3.76(S D=1.93)$; $C B T 2.04(S D=1.29)$, and skill level ratings were MET 4.67 $(\mathrm{SD}=.77)$ and $\mathrm{CBT} 3.00(\mathrm{SD}=.92)$

Dose: $>85 \%$ of participants attended during the last treatment week. Int youth earnings were about $55 \%$ of maximum. Int parents implemented the SMC on average 8.5 of the 11 weeks the contract was active, and administered about 3 saliva alcohol tests per week on average. Both Ctrl and Int teens attended less than half of the continuing care visits. Int parents attended 1 continuing care session, on average

Duration/frequency: 14 weeks plus additional 12 weeks of urine testing

Control: MET/CBT and attendance-based incentives for youth. Parents attended first session and were contacted weekly to report on youth substance but did not receive PT

Outcomes

Length of follow-up: 36 wks (post intervention)

\section{Alcohol outcomes:}

Past 12 week frequency of use measured at 12, 24, and 36 wks to calculate 36 wk total (\% of days)

Funding and Declared Conflicts of Interest
NIH Grants R01AA016917, R01DA015186, UL1TR001086, and P30DA029926. No information or declarations about potential conflicts of interest

Notes 
Stanger 2017 (Continued)

Risk of bias

\begin{tabular}{|c|c|c|}
\hline Bias & Authors' judgement & Support for judgement \\
\hline $\begin{array}{l}\text { Random sequence genera- } \\
\text { tion (selection bias) }\end{array}$ & Low risk & Minimum likelihood of allocation \\
\hline $\begin{array}{l}\text { Allocation concealment } \\
\text { (selection bias) }\end{array}$ & Unclear risk & Not described \\
\hline $\begin{array}{l}\text { Blinding of participants } \\
\text { and personnel (perfor- } \\
\text { mance bias) } \\
\text { All outcomes }\end{array}$ & High risk & Blinding not possible due to the nature of the intervention \\
\hline $\begin{array}{l}\text { Blinding of outcome as- } \\
\text { sessment (detection bias) } \\
\text { All outcomes }\end{array}$ & High risk & Self-reported \\
\hline $\begin{array}{l}\text { Incomplete outcome data } \\
\text { (attrition bias) } \\
\text { All outcomes }\end{array}$ & High risk & $\begin{array}{l}\text { Attrition high, some differential attrition by group apparent but significance } \\
\text { unclear }\end{array}$ \\
\hline $\begin{array}{l}\text { Selective reporting (re- } \\
\text { porting bias) }\end{array}$ & Unclear risk & No protocol or clinical trial registry available \\
\hline Other bias & Low risk & Contamination not described and is unlikely \\
\hline Recruitment to cluster & Unclear risk & Not applicable \\
\hline Baseline imbalances & Unclear risk & Not applicable \\
\hline Loss of clusters & Unclear risk & Not applicable \\
\hline Incorrect analysis & Unclear risk & Not applicable \\
\hline $\begin{array}{l}\text { Compatability with indi- } \\
\text { vidually randomised trials } \\
\text { (herd effect) }\end{array}$ & Unclear risk & Not applicable \\
\hline
\end{tabular}

Stevens 2002

\begin{tabular}{ll}
\hline Methods & Design: cluster RCT \\
& Follow-up: $12,24, \& 36$ mo (post randomisation) \\
& Attrition: $22 \%(12 \mathrm{mo})$ \\
& Unit of randomisation: paediatric clinic \\
& Clustering effect adjusted: yes \\
\hline Participants & N of clusters (subjects) randomised: \\
Int 1: 6 (NR) \\
Int 2: 6 (NR) \\
Total N: 12 (3496) \\
N of clusters (subjects) at 36 mo: \\
\hline
\end{tabular}


Stevens 2002 (Continued)

Int 1: 6 (NR)

Int 2: 6 (NR)

Total N: $12(2183)$

Age: mean: $11(\mathrm{SD}=0.8)$

Sex (male): $50 \%$ to $54 \%$

Ethnicity: NR

Country: USA

\section{Interventions Number of experimental conditions: 2}

Description of Intervention 1: family-based programme mediated through paediatric primary care clinician that included signing a contract with a clinician and engaging child and parent in discussions and communication about alcohol and tobacco smoking; later components included clinician's letter, newsletters of reinforcement, and bi-annual telephone calls

Description of Intervention 2: family-based programme mediated through paediatric primary care clinician that included signing a contract with a clinician and engaging child and parent in discussions and communication about gun safety, seatbelt use, bicycle helmet use; later components included clinician's letter, newsletters of reinforcement, and bi-annual telephone calls

Type of intervention: universal

Focus/target: Int 1 - alcohol and tobacco, Int 2 - gun safety, seatbelt use, bicycle helmet use Fidelity: all paediatricians and nurse practitioners in every practice were trained during a 3 hour session. Compliance was tracked through chart audit; over $99 \%$ of participant charts were labelled with the research project identification sticker and contained a contract. 95\% of children had returned for subsequent visits, $47 \%$ of visits in safety sites and $51 \%$ in alcohol/tobacco sites had a documented prevention message

Dose: at $36 \mathrm{mo}, 93 \%$ of parents and $71 \%$ of children had read at least $50 \%$ of the newsletters

Duration/frequency: 36 mo

Control: not applicable

Outcomes Length of follow-up: 12, 24, \& 36 mo (post randomisation)

\section{Alcohol outcomes:}

Alcohol ever drinker (yes/no)

\section{Funding and Declared} Conflicts of Interest
National Institute of Alcohol and Alcohol Abuse grant AA08946. No information or declarations about potential conflicts of interest

\section{Notes}

\section{Risk of bias}

\begin{tabular}{lll}
\hline Bias & Authors' judgement & Support for judgement \\
\hline $\begin{array}{l}\text { Random sequence genera- } \\
\text { tion (selection bias) }\end{array}$ & Low risk & Computer generated \\
\hline $\begin{array}{l}\text { Allocation concealment } \\
\text { (selection bias) }\end{array}$ & Unclear risk & Not described \\
\hline
\end{tabular}

Blinding of participants High risk $\quad$ Blinding not possible due to the nature of the intervention
and personnel (perfor-

$\begin{array}{lll}\text { Blinding of outcome as- } & \text { High risk } & \text { Self-reported } \\ \text { sessment (detection bias) } & \end{array}$


Stevens 2002 (Continued)

All outcomes

\begin{tabular}{|c|c|c|}
\hline $\begin{array}{l}\text { Incomplete outcome data } \\
\text { (attrition bias) } \\
\text { All outcomes }\end{array}$ & High risk & Attrition high, differential attrition not described \\
\hline $\begin{array}{l}\text { Selective reporting (re- } \\
\text { porting bias) }\end{array}$ & Unclear risk & No protocol or clinical trial registry available \\
\hline Other bias & Low risk & Contamination not described and is unlikely \\
\hline Recruitment to cluster & High risk & Clusters randomised, then participants recruited \\
\hline Baseline imbalances & Low risk & Baseline imbalances accounted for in analysis \\
\hline Loss of clusters & Low risk & No report of loss of entire clusters \\
\hline Incorrect analysis & Low risk & Analysis adjusted for clustering \\
\hline $\begin{array}{l}\text { Compatability with indi- } \\
\text { vidually randomised trials } \\
\text { (herd effect) }\end{array}$ & Unclear risk & Unable to determine if herd effect exists \\
\hline
\end{tabular}

\section{Stormshak 2011}

\begin{tabular}{ll}
\hline Design: RCT \\
Follow-up: $1,2, \& 3$ yrs (post randomisation) \\
Attrition: $19 \%(3$ yrs) \\
Unit of randomisation: family \\
\hline N of subjects randomised: \\
Int: 386 \\
Ctrl: 207 \\
Total N: 593 \\
N of subjects at 3 yrs: \\
Int: NR \\
Ctrl: NR \\
Total N: 481 \\
Age: mean: 11.88 \\
Sex (male): $51 \%$ \\
Ethnicity: $16 \%$ African American, $18 \%$ Latino/Hispanic, $36 \%$ White, $3 \%$ American Indian, $8 \%$ Asian, $19 \%$ \\
bi-racial/mixed ethnicity \\
Country: USA
\end{tabular}

Interventions

\section{Number of experimental conditions: 2}

Description of Intervention: Family Check-Up (FCU) involved 3 brief sessions with parents including motivational interviewing, video family interaction, assessment, and feedback

Type of intervention: selective (low SES/at-risk schools)

Focus/target: preventing an increase in both problem behaviours and substance use during the middle school years Fidelity: NR 
Stormshak 2011 (Continued)

Dose: $51 \%(n=197)$ received consultation from a parent consultant, $42 \%(n=163)$ received the full FCU intervention; among families receiving FCU, $29 \%$ received additional follow-up support after feedback, and the average intervention family received 146 minutes (or 2.5 hours) of intervention time

Duration/frequency: 3 sessions

Control: no programme

Outcomes

Length of follow-up: $1,2, \& 3$ yrs (post randomisation)

\section{Alcohol outcomes:}

Alcohol use - How many alcoholic drinks did you have in the last month?

Funding and Declared Conflicts of Interest
Funding from the National Insitute of Drug Abuse (NIDA). No information or declarations about potential conflicts of interest

\section{Notes}

\section{Risk of bias}

\begin{tabular}{|c|c|c|}
\hline Bias & Authors' judgement & Support for judgement \\
\hline $\begin{array}{l}\text { Random sequence genera- } \\
\text { tion (selection bias) }\end{array}$ & Unclear risk & Not described \\
\hline $\begin{array}{l}\text { Allocation concealment } \\
\text { (selection bias) }\end{array}$ & Unclear risk & Not described \\
\hline $\begin{array}{l}\text { Blinding of participants } \\
\text { and personnel (perfor- } \\
\text { mance bias) } \\
\text { All outcomes }\end{array}$ & High risk & Blinding not possible due to the nature of the intervention \\
\hline $\begin{array}{l}\text { Blinding of outcome as- } \\
\text { sessment (detection bias) } \\
\text { All outcomes }\end{array}$ & High risk & Self-reported \\
\hline $\begin{array}{l}\text { Incomplete outcome data } \\
\text { (attrition bias) } \\
\text { All outcomes }\end{array}$ & Unclear risk & $\begin{array}{l}\text { Attrition low, differential attrition not described, missing data managed by full } \\
\text { information maximum likelihood estimation }\end{array}$ \\
\hline $\begin{array}{l}\text { Selective reporting (re- } \\
\text { porting bias) }\end{array}$ & Unclear risk & No protocol or clinical trial registry available \\
\hline Other bias & Low risk & Contamination not described and is unlikely \\
\hline Recruitment to cluster & Unclear risk & Not applicable \\
\hline Baseline imbalances & Unclear risk & Not applicable \\
\hline Loss of clusters & Unclear risk & Not applicable \\
\hline Incorrect analysis & Unclear risk & Not applicable \\
\hline $\begin{array}{l}\text { Compatability with indi- } \\
\text { vidually randomised trials } \\
\text { (herd effect) }\end{array}$ & Unclear risk & Not applicable \\
\hline
\end{tabular}


Valdez 2013

\begin{tabular}{ll}
\hline Methods & Design: RCT \\
& Follow-up: immediate post-test \& 6 mo (post randomisation) \\
& Attrition: $42 \%(6 \mathrm{mo})$ \\
& Unit of randomisation: family
\end{tabular}

\begin{tabular}{ll}
\hline Participants & N subjects randomised: \\
Int: 96 \\
Ctrl: 104 \\
Total N: 200 \\
N of subjects at 6 mo: \\
Int: 54 \\
Ctrl: 62 \\
Total N: 116 \\
Age: mean: 15.25 (SD = NR); range 12 to 17 yrs \\
Sex (male): $49 \%$ \\
Ethnicity: $100 \%$ Mexican American \\
Country: USA
\end{tabular}

Interventions

\section{Number of experimental conditions: 2}

Description of Intervention 1: adapted Brief Family Strategic Therapy (BFST) involved 4 components: (1) organise a counsellor-family work team in developing a therapeutic alliance; (2) diagnose family strengths and problem relations with emphasis on supportive family relations; (3) develop a change strategy to capitalise on strengths and to correct problematic family relations; and (4) implement change strategies and reinforce family behaviours that sustain new levels of family competence

Type of intervention: indicated (gang-affiliated youth identified as abusing alcohol and drugs)

Focus/target: correct problematic family relations; adhere to change strategies; support communication with school, gang diversion, HIV/STD prevention

Fidelity: periodically monitored by evaluation staff members using a fidelity checklist, $\%$ NR

Dose: NR

Duration/frequency: 12 to 16 sessions weekly, 60 to 90 min per session

Control: received usual care; referrals to social and behavioural health services and substance abuse counselling upon request

\section{Outcomes}

Length of follow-up: immediate post-test $\& 6 \mathrm{mo}$ (post randomisation)

\section{Alcohol outcomes:}

Number of days in past 30 days on which adolescent used alcohol

Funding and Declared Conflicts of Interest
Funding received through the Substance Abuse and Mental Health Services Administration and Drug Treatment for Gang Affiliated Hispanic Adolescents. Study authors declared no conflicts of interest

\section{Notes}

\begin{tabular}{lll}
\hline Risk of bias & \\
\hline Bias & Authors' judgement & Support for judgement \\
\hline $\begin{array}{l}\text { Random sequence genera- } \\
\text { tion (selection bias) }\end{array}$ & Low risk & Computerised random number generator \\
\hline $\begin{array}{l}\text { Allocation concealment } \\
\text { (selection bias) }\end{array}$ & Unclear risk & Not described \\
\hline
\end{tabular}


Valdez 2013 (Continued)

\begin{tabular}{|c|c|c|}
\hline $\begin{array}{l}\text { Blinding of participants } \\
\text { and personnel (perfor- } \\
\text { mance bias) } \\
\text { All outcomes }\end{array}$ & High risk & Blinding not possible due to the nature of the intervention \\
\hline $\begin{array}{l}\text { Blinding of outcome as- } \\
\text { sessment (detection bias) } \\
\text { All outcomes }\end{array}$ & High risk & Self-reported \\
\hline $\begin{array}{l}\text { Incomplete outcome data } \\
\text { (attrition bias) } \\
\text { All outcomes }\end{array}$ & High risk & $\begin{array}{l}\text { Attrition high, differential attrition between groups apparent but significance } \\
\text { unclear, attrition due to discontinued participation, inability to locate and re- } \\
\text { locate to other towns, intention-to-treat analysis cited }\end{array}$ \\
\hline $\begin{array}{l}\text { Selective reporting (re- } \\
\text { porting bias) }\end{array}$ & Unclear risk & No protocol or clinical trial registry available \\
\hline Other bias & Low risk & Contamination not described and is unlikely \\
\hline Recruitment to cluster & Unclear risk & Not applicable \\
\hline Baseline imbalances & Unclear risk & Not applicable \\
\hline Loss of clusters & Unclear risk & Not applicable \\
\hline Incorrect analysis & Unclear risk & Not applicable \\
\hline $\begin{array}{l}\text { Compatability with indi- } \\
\text { vidually randomised trials } \\
\text { (herd effect) }\end{array}$ & Unclear risk & Not applicable \\
\hline
\end{tabular}

Werch 2008

\begin{tabular}{|c|c|}
\hline Methods & $\begin{array}{l}\text { Design: } \mathrm{RCT} \\
\text { Follow-up: } 4 \text { mo (post intervention) } \\
\text { Attrition: } 5 \% \text { to } 8 \%(4 \mathrm{mo}) \\
\text { Unit of randomisation: student }\end{array}$ \\
\hline Participants & $\begin{array}{l}\text { N of subjects randomised: } \\
\text { Int: NR } \\
\text { Ctrl: NR } \\
\text { Total N: } 684 \\
\text { N of subjects at } 19 \text { wks: } \\
\text { Int: } 182 \\
\text { Ctrl: } 202 \\
\text { Total N: } 384 \\
\text { Age: mean: } 15.24 \text { (SD = 1.09) } \\
\text { Sex (male): } 44 \% \\
\text { Ethnicity: } 49.6 \% \text { White, } 21.2 \% \text { African American, } 29.3 \% \text { Other } \\
\text { Country: USA }\end{array}$ \\
\hline Interventions & $\begin{array}{l}\text { Number of experimental conditions: } 2 \\
\text { Description of Intervention 1: } 8.5 \times 11 \text { inch 1-sided parent postcard (adopted from Project Sport Con- } \\
\text { sultation); a brief image-based print-mediated parent/caregiver message about communication on fit- } \\
\text { ness promotion and avoidance of alcohol. A series of } 3 \text { postcards were mailed }\end{array}$ \\
\hline
\end{tabular}


Description of Intervention 2: $8.5 \times 11$ inch 2-sided adolescent flier integrating physical activity and other health-promoting behaviours. A series of 3 fliers were mailed with similar but shorter messages than those on the parental postcard, but with commercial quality images of healthy and active youth with brief fitness and alcohol avoidance messages

Type of intervention: universal

Focus/target: alcohol use Fidelity: NR

Dose: $65 \%$ of parents received at least 1 postcard and $53 \%$ received all 3 postcards; corresponding proportions of adolescents receiving fliers were $70 \%$ and $59 \%$, respectively; $95 \%$ of parents talked to their children about the postcard, and $91 \%$ of teens liked the fliers

Duration/frequency: 3 wks (1 postcard or flier mailed per week)

Control: not applicable

Length of follow-up: 4 mo (post intervention)
Alcohol outcomes:
Stage of alcohol initiation based on stages of change theory
Length of alcohol use (ranging from 30 days or less to 6 months or more)
30 day alcohol frequency
30 day alcohol quantity
30 day alcohol heavy use
Alcohol problems

Funding and Declared Conflicts of Interest
This manuscript was supported in part by grants from the National Institute on Alcohol Abuse and Alcoholism (Grant \#AA9283) and from the National Institute on Drug Abuse (Grant \#DA018872 and \#DA019172). No information or declarations about potential conflicts of interest

Notes

\section{Risk of bias}

\begin{tabular}{lll}
\hline Bias & Authors' judgement & Support for judgement \\
\hline $\begin{array}{l}\text { Random sequence genera- } \\
\text { tion (selection bias) }\end{array}$ & Unclear risk & Not described \\
\hline $\begin{array}{l}\text { Allocation concealment } \\
\text { (selection bias) }\end{array}$ & Unclear risk & Not described \\
\hline $\begin{array}{l}\text { Blinding of participants } \\
\text { and personnel (perfor- } \\
\text { mance bias) } \\
\text { All outcomes }\end{array}$ & High risk & Blinding not possible due to the nature of the intervention \\
\hline $\begin{array}{l}\text { Blinding of outcome as- } \\
\text { sessment (detection bias) } \\
\text { All outcomes }\end{array}$ & High risk & Self-reported \\
\hline $\begin{array}{l}\text { Incomplete outcome data } \\
\text { (attrition bias) } \\
\begin{array}{l}\text { All outcomes } \\
\hline\end{array}\end{array}$ & Low risk & Attrition low, no differential attrition by group, imputation not described \\
\hline
\end{tabular}


Werch 2008 (Continued)

\begin{tabular}{|c|c|c|}
\hline $\begin{array}{l}\text { Selective reporting (re- } \\
\text { porting bias) }\end{array}$ & Unclear risk & No protocol or clinical trial registry available \\
\hline Other bias & Low risk & Contamination not described and is unlikely \\
\hline Recruitment to cluster & Unclear risk & Not applicable \\
\hline Baseline imbalances & Unclear risk & Not applicable \\
\hline Loss of clusters & Unclear risk & Not applicable \\
\hline Incorrect analysis & Unclear risk & Not applicable \\
\hline $\begin{array}{l}\text { Compatability with indi- } \\
\text { vidually randomised trials } \\
\text { (herd effect) }\end{array}$ & Unclear risk & Not applicable \\
\hline
\end{tabular}

Winters 2012

\begin{tabular}{ll}
\hline Methods & Design: $\mathrm{RCT}$ \\
& Follow-up: $6 \& 12 \mathrm{mo}$ (post randomisation) \\
& Attrition: $1.3 \%(6 \mathrm{mo}), 9.8 \%(12 \mathrm{mo})$ \\
& Unit of randomisation: student
\end{tabular}

Participants

$\mathrm{N}$ of subjects randomised:

Int 1: 136

Int 2: 123

Ctrl: 56

Total N: 315

$\mathrm{N}$ of subjects at $12 \mathrm{mo}$ :

Int 1: 122

Int 2: 114

Ctrl: 48

Total N: 284

Age: mean: $16.3(\mathrm{SD}=1.4)$; range 12 to $18 \mathrm{yrs}$

Sex (male): $52 \%$

Ethnicity: $68 \%$ White

Country: USA

\section{Number of experimental conditions: 3}

Description of Intervention 1: Brief Intervention Adolescent (BI-A) involved 60 min sessions using motivational interviewing. Session 1 included eliciting information about students' alcohol and other drug use and related consequences, assessing their willingness to change, examining the pros and cons of their use via the decisional balance exercise, and discussing what goals for change the student would like to select and pursue. Session 2 included monitoring students' progress in achieving goals, identifying high-risk situations associated with drug use triggers, discussing strategies to deal with social pressures to use drugs, assessing again willingness to change, and negotiating long-term goals

Description of Intervention 2: Brief Intervention Adolescent \& Parent (BI-AP) involved 60 min sessions using motivational interviewing, sessions 1 \& 2 same as for BI-A but with youth only, session 3 for parent. The third session (for BI-AP) involves delivering the same MI interviewing style to the primary parent or guardian and addressing their son's or daughter's substance use problem, providing par- 
Winters 2012 (Continued)

ent monitoring and supervision to promote progress towards their child's intervention goals, and discussing healthy drug use behaviours and attitudes for the parent

Type of intervention: indicated (youth identified as abusing alcohol and drugs)

Focus/target: drug and alcohol use, parent monitoring

Fidelity: therapists covered $98 \%$ of key components of the intervention sessions

Dose: three students in the BI-AP group completed just 1 of their adolescent sessions (although the parent session was completed in all of these cases), and 2 parents in the BIAP group did not complete their single session (although the adolescent completed his/her sessions)

Duration/frequency: BI-A - 2 sessions with adolescents only, BIAP - 2 sessions with adolescents only and 1 additional parent session; sessions 1 and 2 were separated by 7 to 10 days

Control: no programme

Length of follow-up: $6 \& 12$ mo (post randomisation)
Alcohol outcomes:
Number of alcohol use days in prior 90 days
Number of alcohol abuse symptoms present in prior 90 days
Number of alcohol dependency symptoms present in prior 90 days
Total abstinence from alcohol for prior 90 days (\%)
Alcohol abuse symptoms absent for prior 6 months (\%)
Alcohol dependency symptoms absent for prior 6 months (\%)

Funding and Declared

Funding for the project provided by the National Insitute on Health. No information or declarations Conflicts of Interest about potential conflicts of interest

Notes

\section{Risk of bias}

\begin{tabular}{lll}
\hline Bias & Authors' judgement & Support for judgement \\
\hline $\begin{array}{l}\text { Random sequence genera- } \\
\text { tion (selection bias) }\end{array}$ & Low risk & Urn randomisation procedure \\
\hline $\begin{array}{l}\text { Allocation concealment } \\
\text { (selection bias) }\end{array}$ & Unclear risk & Not described \\
\hline $\begin{array}{l}\text { Blinding of participants } \\
\text { and personnel (perfor- } \\
\text { mance bias) }\end{array}$ & High risk & Blinding not possible due to the nature of the intervention \\
All outcomes & \\
\hline $\begin{array}{l}\text { Blinding of outcome as- } \\
\text { sessment (detection bias) } \\
\text { All outcomes }\end{array}$ & High risk & Self-reported \\
\hline $\begin{array}{l}\text { Incomplete outcome data } \\
\text { (attrition bias) } \\
\text { All outcomes }\end{array}$ & Low risk & $\begin{array}{l}\text { Atrrition low, no differential attrition between groups, mention of handling of } \\
\text { missing data not described }\end{array}$ \\
\hline $\begin{array}{l}\text { Selective reporting (re- } \\
\text { porting bias) }\end{array}$ & Unclear risk & No protocol or clinical trial registry available \\
\hline \hline
\end{tabular}


Winters 2012 (Continued)

\begin{tabular}{|c|c|c|}
\hline Other bias & Low risk & Contamination not described and is unlikely \\
\hline Recruitment to cluster & Unclear risk & Not applicable \\
\hline Baseline imbalances & Unclear risk & Not applicable \\
\hline Loss of clusters & Unclear risk & Not applicable \\
\hline Incorrect analysis & Unclear risk & Not applicable \\
\hline $\begin{array}{l}\text { Compatability with indi- } \\
\text { vidually randomised trials } \\
\text { (herd effect) }\end{array}$ & Unclear risk & Not applicable \\
\hline
\end{tabular}

Wolchik 2002

$\begin{array}{ll}\text { Methods } & \text { Design: RCT } \\ & \text { Follow-up: } 6 \& 15 \mathrm{yrs} \text { (post randomisation) } \\ & \text { Attrition: } 9 \% \text { (6 yrs), 19\% (15 yrs) } \\ & \text { Unit of randomisation: family }\end{array}$

Participants N of subjects randomised:

Int 1: 83

Int 2: 81

Ctrl: 76

Total N: 240

$\mathrm{N}$ of subjects at $15 \mathrm{yrs}$ :

Int 1+ Int 2: 134

Ctrl: 60

Total N: 194

Age: mean: $10.7(\mathrm{SD}=1.1)$; range 9 to $12 \mathrm{yrs}$

Sex (male): $50.5 \%$

Ethnicity: $89 \%$ Non-Hispanic White

Country: USA

\section{Number of experimental conditions: 3}

Description of Intervention 1 + 2: New Beginnings programme with 2 intervention arms analysed together: Mother Plus Child Program (MPCP) and Mother Program (MP) - 11 group sessions including videotaped modelling and role-plays used to teach skills, and weekly homework assignments focused on practising programme skills. Particpants in MP also received 2 individual sessions to tailor the programme to individual needs.

Type of intervention: selective (adolescents with divorced parents)

Focus/target: MP programme - improving the quality of the mother-child relationship, providing effective discipline, increasing fathers' access to children, and reducing interparental conflict; MPCP program - developing effective coping and reducing negative thoughts about divorce stressors Fidelity: independent raters scored each programme segment using videotapes ( $1=$ not at all complete to $3=$ complete); mean completion was $2.86(S D=0.39)$ and $3.00(S D=0.02)$ for mother and child sessions, respectively

Dose: mothers attended an average of $77 \%$ of sessions, and children $78 \%$ of sessions 
Duration/frequency: groups met for 11 sessions for 1.75 hours per session (MP+MPCP) plus 2 individual sessions (MP only)

Control: 3 books about children's divorce adjustment and a syllabus to guide reading - 1 month intervals

\begin{tabular}{|c|c|c|}
\hline \multirow[t]{7}{*}{ Outcomes } & \multicolumn{2}{|c|}{ Length of follow-up: 15 yrs (post randomisation) } \\
\hline & \multicolumn{2}{|l|}{ Alcohol outcomes: } \\
\hline & \multicolumn{2}{|c|}{ Age started regular drinking } \\
\hline & \multicolumn{2}{|l|}{ Binge drinking past year } \\
\hline & \multicolumn{2}{|c|}{ Alcohol use past month (number of occasions used) } \\
\hline & \multicolumn{2}{|c|}{ Alcohol withdrawal, dependence, abuse diagnosis (diagnostic interview), past $9 \mathrm{yrs}$} \\
\hline & \multicolumn{2}{|c|}{ Alcohol withdrawal, dependence, abuse diagnosis (diagnostic interview), past $15 \mathrm{yrs}$} \\
\hline $\begin{array}{l}\text { Funding and Declared } \\
\text { Conflicts of Interest }\end{array}$ & \multicolumn{2}{|c|}{$\begin{array}{l}\text { Funding for study provided by National Institutes of Mental Health. No information or declarations } \\
\text { about potential conflicts of interest }\end{array}$} \\
\hline \multicolumn{3}{|l|}{ Notes } \\
\hline \multicolumn{3}{|l|}{ Risk of bias } \\
\hline Bias & Authors' judgement & Support for judgement \\
\hline $\begin{array}{l}\text { Random sequence genera- } \\
\text { tion (selection bias) }\end{array}$ & Low risk & $\begin{array}{l}\text { Randomisation software, developed by an individual not affiliated with the re- } \\
\text { search project, was designed to avoid large differences across experimental } \\
\text { conditions as families were sequentially enrolled }\end{array}$ \\
\hline $\begin{array}{l}\text { Allocation concealment } \\
\text { (selection bias) }\end{array}$ & Unclear risk & Not described \\
\hline $\begin{array}{l}\text { Blinding of participants } \\
\text { and personnel (perfor- } \\
\text { mance bias) } \\
\text { All outcomes }\end{array}$ & High risk & Blinding not possible due to the nature of the intervention \\
\hline $\begin{array}{l}\text { Blinding of outcome as- } \\
\text { sessment (detection bias) } \\
\text { All outcomes }\end{array}$ & High risk & Self-reported \\
\hline $\begin{array}{l}\text { Incomplete outcome data } \\
\text { (attrition bias) } \\
\text { All outcomes }\end{array}$ & Low risk & Attrition low, no differential attrition by group, intention-to-treat analysis cited \\
\hline $\begin{array}{l}\text { Selective reporting (re- } \\
\text { porting bias) }\end{array}$ & Unclear risk & No protocol or clinical trial registry available \\
\hline Other bias & Low risk & Contamination not described and is unlikely \\
\hline Recruitment to cluster & Unclear risk & Not applicable \\
\hline Baseline imbalances & Unclear risk & Not applicable \\
\hline Loss of clusters & Unclear risk & Not applicable \\
\hline
\end{tabular}


Wolchik 2002 (Continued)

\begin{tabular}{lll} 
Incorrect analysis & Unclear risk & Not applicable \\
\hline $\begin{array}{l}\text { Compatability with indi- } \\
\text { vidually randomised trials } \\
\text { (herd effect) }\end{array}$ & Unclear risk & Not applicable \\
\hline
\end{tabular}

Wu 2003

\begin{tabular}{ll} 
Methods & Design: cluster RCT \\
& Follow-up: $6 \& 12 \mathrm{mo}$ (post intervention) \\
& Attrition: $26 \%$ (6 mo), $29 \%$ (12 mo) \\
& Unit of randomisation: community centre \\
& Clustering effect adjusted: no \\
\hline
\end{tabular}

Participants N of clusters (subjects) randomised:

Int 1: NR (496)

Int 2: NR (321)

Total N: 35 (817)

$\mathrm{N}$ of clusters (subjects) at $12 \mathrm{mo}$ :

Int 1: NR (337)

Int 2: NR (243)

Total N: 35 (580)

Age: median: 14 ; range 12 to 16 yrs

Sex (male): $42 \%$

Ethnicity: 100\% African American

Country: USA

Interventions

\section{Number of experimental conditions: 2}

Description of Intervention 1: Focus On Kids + Informed Parents \& Children Together (FOK + IMPACT) FOK = HIV risk reduction intervention delivered by a group leader with an assistant group leader; 8 sessions - sex, drugs, alcohol, drug-selling, group session with youths. IMPACT = 20 minute video on parental monitoring \& communication in the home, role-playing, discussion. Boosters for FOK were conducted immediately after 6 -month follow-up and at 10 months among youths only. Booster sessions consist of a review of activities that had been done in the primary sessions and the addition of a few new activities that reviewed the content of the original programme

Description of Intervention 2: Focus On Kids (FOK), as described for Intervention 1

Type of intervention: selective (low-income areas)

Focus/target: FOK aims to reduce the risk of HIV, and IMPACT aims to increase parental monitoring and communication

Fidelity: NR

Dose: NR

Duration/frequency: FOK - 8 sessions; IMPACT - 20 min video + 2 instructor-led role-plays; Boosters at 6 \& 10 months amongst youth

Control: not applicable

\section{Outcomes}

\section{Length of follow-up: $6 \& 12$ mo (post intervention)}

\section{Alcohol outcomes:}

Drank alcohol (yes/no) in previous 6 months 
Wu 2003 (Continued)

Funding and Declared Conflicts of Interest
Funding provided through National Institutes of Mental Health. No information or declarations about potential conflicts of interest

Notes

\section{Risk of bias}

\begin{tabular}{|c|c|c|}
\hline Bias & Authors' judgement & Support for judgement \\
\hline $\begin{array}{l}\text { Random sequence genera- } \\
\text { tion (selection bias) }\end{array}$ & Unclear risk & Not described \\
\hline $\begin{array}{l}\text { Allocation concealment } \\
\text { (selection bias) }\end{array}$ & Unclear risk & Not described \\
\hline $\begin{array}{l}\text { Blinding of participants } \\
\text { and personnel (perfor- } \\
\text { mance bias) } \\
\text { All outcomes }\end{array}$ & High risk & $\begin{array}{l}\text { Blinding of participants and personnel not possible due to the nature of the in- } \\
\text { tervention. }\end{array}$ \\
\hline $\begin{array}{l}\text { Blinding of outcome as- } \\
\text { sessment (detection bias) } \\
\text { All outcomes }\end{array}$ & High risk & $\begin{array}{l}\text { Self-reported; blinding of participants and personnel not possible due to the } \\
\text { nature of the intervention }\end{array}$ \\
\hline $\begin{array}{l}\text { Incomplete outcome data } \\
\text { (attrition bias) } \\
\text { All outcomes }\end{array}$ & High risk & $\begin{array}{l}\text { Attrition high, no differential attrition between groups, no differential attrition } \\
\text { between completers and non-completers }\end{array}$ \\
\hline $\begin{array}{l}\text { Selective reporting (re- } \\
\text { porting bias) }\end{array}$ & Unclear risk & No protocol or clinical trial registry available \\
\hline Other bias & Low risk & Contamination not described and is unlikely \\
\hline Recruitment to cluster & Low risk & Clusters randomised after participant recruitment \\
\hline Baseline imbalances & Low risk & Baseline imbalances accounted for in analysis \\
\hline Loss of clusters & Low risk & No report of loss of entire clusters \\
\hline Incorrect analysis & Low risk & $\begin{array}{l}\text { Analysis not sufficiently adjusted for clustering; review authors performed an } \\
\text { alternative adjustment }\end{array}$ \\
\hline $\begin{array}{l}\text { Compatability with indi- } \\
\text { vidually randomised trials } \\
\text { (herd effect) }\end{array}$ & Unclear risk & Unable to determine if herd effect exists \\
\hline
\end{tabular}

\section{Wurdak 2017}

\begin{tabular}{ll}
\hline Methods & Design: RCT \\
& Follow-up: 4 wks post randomisation (immediately post intervention) \\
& Attrition: $44 \%$ adolescents, $26 \%$ parents (4 wks) \\
& Unit of randomisation: parent \\
& Clustering effect adjusted: not applicable \\
\hline Participants & N of subjects randomised:
\end{tabular}


Wurdak 2017 (Continued)

Int: 310 parents; 153 adolescents

Ctrl: 321 parents; 158 adolescents

Total N: 631 parents; 311 adolescents

$\mathrm{N}$ of subjects at 4 wks:

Int: 231 parents; 84 adolescents

Ctrl: 236 parents; 89 adolescents

Total N: 467 parents; 173 adolescents

Age: mean $15.0(\mathrm{SD}=1.1)$

Sex (male): $60.8 \%$

Ethnicity: NR

Country: Germany

Interventions

Number of experimental conditions: 2

Description of Intervention: 4 weekly emails to parents with a short introductory text and a video clip, a timetable, and a 4-page PDF document containing different chapters. Chapters contain information and practical parenting advice for everyday life and an exercise focusing on basic skills and parenting variables

Type of intervention: universal

Focus/target: excessive alcohol consumption, parents' alcohol-related knowledge, self-efficacy, and parenting skills

Fidelity: NR

Dose: four weekly emails, \% NR

Duration/frequency: 4 weeks

Control: waitlist control

Outcomes Length of follow-up: 4 weeks post randomisation (immediately post intervention)

\section{Alcohol outcomes:}

Frequency of drinking (last 30 days)

Frequency of heavy episodic drinking (last 30 days, 5 or more alcoholic drinks)

Frequency of drunkenness (last 30 days; how many occasions have you been intoxicated from drinking alcoholic beverages...)

Funding and Declared

The study received financial support from the University of Bamberg, the Federal Health Ministry, in

Conflicts of Interest Bavaria (grant nos: 55.2-2682.01-4/12 and 55.2-2682.01-4/13) and the health insurer AOK Study authors declare no conflicts of interest

\section{Notes}

\section{Risk of bias}

\begin{tabular}{lll}
\hline Bias & Authors' judgement & Support for judgement \\
\hline $\begin{array}{l}\text { Random sequence genera- } \\
\text { tion (selection bias) }\end{array}$ & Unclear risk & Not described \\
\hline $\begin{array}{l}\text { Allocation concealment } \\
\text { (selection bias) }\end{array}$ & Unclear risk & Not described \\
\hline
\end{tabular}

Blinding of participants

High risk

Blinding not possible due to the nature of the intervention

and personnel (perfor-

mance bias) 
Wurdak 2017 (Continued)

All outcomes

\begin{tabular}{|c|c|c|}
\hline $\begin{array}{l}\text { Blinding of outcome as- } \\
\text { sessment (detection bias) } \\
\text { All outcomes }\end{array}$ & High risk & Self-reported \\
\hline $\begin{array}{l}\text { Incomplete outcome data } \\
\text { (attrition bias) } \\
\text { All outcomes }\end{array}$ & High risk & $\begin{array}{l}\text { Attrition high, differential attrition by group minimal, some differential attri- } \\
\text { tion by characteristics }\end{array}$ \\
\hline $\begin{array}{l}\text { Selective reporting (re- } \\
\text { porting bias) }\end{array}$ & Low risk & $\begin{array}{l}\text { Reported outcomes are consistent with the German clinical trial registry: iden- } \\
\text { tifier: DRKS00007763 }\end{array}$ \\
\hline Other bias & Low risk & Contamination not described and is unlikely \\
\hline Recruitment to cluster & Unclear risk & Not applicable \\
\hline Baseline imbalances & Unclear risk & Not applicable \\
\hline Loss of clusters & Unclear risk & Not applicable \\
\hline Incorrect analysis & Unclear risk & Not applicable \\
\hline $\begin{array}{l}\text { Compatability with indi- } \\
\text { vidually randomised trials } \\
\text { (herd effect) }\end{array}$ & Unclear risk & Not applicable \\
\hline
\end{tabular}

ABI: Abstinence Based Incentives.

BFST: Brief Family Strategic Therapy.

BI-A: Brief Intervention Adolescent.

BI-AP: Brief Intervention Adolescent \& Parent.

BPI: Brief Prevention Intervention.

CAPR: Children and Parent Relations.

CBT: cognitive-behavioural therapy.

CC: classroom-centred.

CDP: CD-ROM + Parent programme.

Ctrl: control.

D.A.R.E.: Drug Abuse Resistance Education.

ESC: Enhanced Standard Care.

ESI: Extended Services Intervention.

FCU: Family Check-Up.

FEI: Family Empowerment Intervention.

FOF: Focus On Families.

FOK: Focus On Kids.

FSP: Family-School Partnership.

FU: follow-up.

HRIDAY: Health-Related Information and Dissemination Among Youth.

IMI: individual motivational interview.

IMPACT: Informed Parents \& Children Together.

Int: intervention.

ISFP: lowa Strengthening Families Program.

LST: life skills training.

MDFT: Multi-Dimensional Family Therapy.

MET: motivational enhancement therapy.

MI: motivational interview.

MITI: Motivational Interviewing Treatment Integrity.

MP: Mother Program.

MPCP: Mother Plus Child Program.

NR: not reported.

Family-based prevention programmes for alcohol use in young people (Review) 
OPP: Orebro Prevention Program.

PDFY: Preparing for Drug Free Years.

PE: psychoeducation.

PI: parent intervention.

PT: parent training.

PWC: Parents Who Care.

PWC-PA: Parents Who Care Parent \& Adolescent format.

PWC-SA: Parents Who Care Self-administered.

$\mathrm{RCT}$ : randomised controlled trial.

SAAF: Strong African American Families programme.

SD: standard deviation.

SES: socioeconomic status.

SFP: Strengthening Families Program.

SI: student intervention.

SMC: Substance Monitoring Contract.

STRIVE: Support To Reunite, Involve and Value Each Other.

Characteristics of excluded studies [ordered by study ID]

\begin{tabular}{|c|c|}
\hline Study & Reason for exclusion \\
\hline Adolfsen 2017 & No alcohol outcome \\
\hline Allen 2007 & No alcohol outcome \\
\hline Andreasson 2007 & Intervention not parent/family \\
\hline Anonymous 2013 & Inappropriate design - not a randomised controlled trial \\
\hline Barrett 2012 & Intervention not parent/family \\
\hline Bauman 2001 & No alcohol outcome \\
\hline Bauman 2001a & No alcohol outcome \\
\hline Biglan 2000 & Intervention not parent/family \\
\hline Bobrowski 2014 & Intervention not parent/family \\
\hline Brody 2004 & No alcohol outcome \\
\hline Brody 2005 & Inappropriate design - no isolation of parent/family intervention \\
\hline Brown 2014 & No alcohol outcome \\
\hline Byrnes 2010 & No alcohol outcome \\
\hline Calabria 2013 & Inappropriate design - not a randomised controlled trial \\
\hline Chilenski 2016 & No alcohol outcome \\
\hline Cohen 1995 & Inappropriate design - not a randomised controlled trial \\
\hline Connell 2007 & Intervention not parent/family \\
\hline Conrod 2009 & Intervention not parent/family \\
\hline
\end{tabular}




\begin{tabular}{|c|c|}
\hline Study & Reason for exclusion \\
\hline Conrod 2011 & Intervention not parent/family \\
\hline de Leeuw 2014 & No alcohol outcome \\
\hline DeGarmo 2009 & Intervention not parent/family \\
\hline Donovan 2012 & Inappropriate participants - age \\
\hline Ennett 2001 & No alcohol outcome \\
\hline Epstein 2008 & No alcohol outcome \\
\hline Faggiano 2007 & Inappropriate design - no isolation of parent/family intervention \\
\hline Fernandez 2011 & Inappropriate participants - age \\
\hline French 2008 & No alcohol outcome \\
\hline Gerrard 2006 & No alcohol outcome \\
\hline Gordon 2008 & Intervention not for alcohol \\
\hline Grossbard 2010 & Inappropriate participants - age \\
\hline Haggerty 2006 & No alcohol outcome \\
\hline Hallgren 2011 & Intervention not parent/family \\
\hline Hawkins 2012 & Intervention not parent/family \\
\hline Hogue 2006 & No alcohol outcome \\
\hline Ingels 2013 & Inappropriate design - no isolation of parent/family intervention \\
\hline Jackson 2016 & No alcohol outcome \\
\hline Jones 2007 & Inappropriate design - no isolation of parent/family intervention \\
\hline Komro 2006 & No alcohol outcome \\
\hline Komro 2017 & Intervention not parent/family \\
\hline Koning 2011 & No alcohol outcome \\
\hline Koning 2012 & No alcohol outcome \\
\hline Koning 2014 & No alcohol outcome \\
\hline Koning 2015 & No alcohol outcome \\
\hline Koning 2016 & No alcohol outcome \\
\hline Kosterman 2001 & No alcohol outcome \\
\hline Koutakis 2008 & Inappropriate design - not a randomised controlled trial \\
\hline
\end{tabular}




\begin{tabular}{|c|c|}
\hline Study & Reason for exclusion \\
\hline Liddle 2001 & No alcohol outcome \\
\hline Litrownik 2000 & No alcohol outcome \\
\hline Lowman 2004 & Inappropriate design - not a randomised controlled trial \\
\hline Malmberg 2014 & Inappropriate design - no isolation of parent/family intervention \\
\hline Mares 2011 & No alcohol outcome \\
\hline McGillicuddy 2015 & Intervention not for alcohol \\
\hline Newton 2012 & Intervention not parent/family \\
\hline Pentz 1989 & Intervention not parent/family \\
\hline Perry 1993 & Intervention not parent/family \\
\hline Perry 1996 & Intervention not parent/family \\
\hline Perry 2000 & Intervention not parent/family \\
\hline Perry 2002 & Intervention not parent/family \\
\hline Romero 2017 & No adolescent alcohol outcomes \\
\hline Rowe 2008 & Inappropriate design - not a randomised controlled trial \\
\hline Shortt 2007 & Intervention not parent/family \\
\hline Simons-Morton 2005 & Intervention not parent/family \\
\hline Simons-Morton 2005a & Intervention not parent/family \\
\hline Spoth $2002 a$ & No alcohol outcome \\
\hline Spoth 2007 & Intervention not parent/family \\
\hline Spoth 2013 & Inappropriate design - no isolation of parent/family intervention \\
\hline Spoth 2014 & Intervention not parent/family \\
\hline Stanton 2004 & No alcohol outcome \\
\hline Stigler 2006 & Intervention not parent/family \\
\hline Teesson 2013 & Intervention not parent/family \\
\hline Tomczyk 2015 & Intervention not parent/family \\
\hline Turrisi 2013 & Inappropriate participants - age \\
\hline University of Minnesota 2015 & No alcohol outcome \\
\hline University of Minnesota 2018 & No alcohol outcome \\
\hline
\end{tabular}




\begin{tabular}{|c|c|}
\hline Study & Reason for exclusion \\
\hline University of Rhode Island 2011 & Intervention not parent/family \\
\hline Valentine 1998 & Intervention not parent/family \\
\hline Verdurmen 2014 & No alcohol outcome \\
\hline Vermeulen-Smit 2014 & No alcohol outcome \\
\hline Veronneau 2016 & Intervention not parent/family \\
\hline Watson 2017 & No alcohol outcome \\
\hline Werch 1998 & Inappropriate design - no isolation of parent/family intervention \\
\hline Werch 1999 & Intervention not parent/family \\
\hline Werch 2003 & Inappropriate design - no isolation of parent/family intervention \\
\hline Werch 2010 & Intervention not parent/family \\
\hline Williams 1995 & No alcohol outcome \\
\hline Williams 2001 & Intervention not parent/family \\
\hline Wolchik 2003 & No alcohol outcome \\
\hline Zanetta 2008 & Inappropriate design - not a randomised controlled trial \\
\hline
\end{tabular}

\section{Characteristics of studies awaiting assessment [ordered by study ID]}

Danielson 2016

\section{Methods}

\section{Participants}

Interventions

\section{Outcomes}

Notes No full text available to determine eligibility

\begin{tabular}{ll}
\hline Ford 2018 & Randomised controlled trial \\
\hline Methods & Adolescents aged 14 to 15 years and their parents \\
\hline Participants & Sex intervention, alcohol intervention, control \\
\hline Onterventions & Ful text not available to determine alcohol outcome reporting
\end{tabular}


Ford 2018 (Continued)
Notes
No full text available to determine eligibility - conference abstract only

Noel 2015

Methods

Participants

Interventions

Outcomes

Notes No full text available to determine eligibility

Winters 2015

Methods

Participants

Interventions

Outcomes

Notes No full text available to determine eligibility

Characteristics of ongoing studies [ordered by study ID]

Allen 2012

\begin{tabular}{ll}
\hline Trial name or title & Immigrant Family Skills-Building to Prevent Tobacco Use in Latino Youth: Study Protocol for a \\
Community-Based Participatory Randomized Controlled Trial
\end{tabular}

\begin{tabular}{|c|c|}
\hline Methods & Randomised controlled trial \\
\hline Participants & 336 Latino families including 1 adolescent aged 10 to 14 years \\
\hline Interventions & $\begin{array}{l}\text { Intervention: the Padres Informados/Jóvenes Preparados (PI/JP) curriculum aims to develop } \\
\text { strong parenting practices and to facilitate relationship-building between parents and youth, while } \\
\text { emphasising Latino cultural values, navigation in multiple cultures, and environmental risks relat- } \\
\text { ed to socioeconomic circumstances }\end{array}$ \\
\hline & Control: waitlist (received intervention 6 months after intervention group) \\
\hline
\end{tabular}

\begin{tabular}{ll}
\hline Outcomes & Past 30 days alcohol use \\
\hline Starting date & NR \\
\hline Contact information & Michele Allen, University of Minnesota; miallen@umn.edu \\
\hline Notes & ClinicalTrials.gov identifier: NCT01442753 \\
\hline \hline
\end{tabular}

Family-based prevention programmes for alcohol use in young people (Review) 
Bukstein 2006

Trial name or title

Home-Based Behavioral Therapy (HBT): Psychosocial Intervention Project for Early Adolescents With Pre- or Early Substance Use Disorder: Phase II

\begin{tabular}{ll}
\hline Methods & Randomised controlled trial \\
\hline Participants & $\begin{array}{l}36 \text { adolescents aged } 11 \text { to } 14 \text { years with a DSM-IV disruptive behaviour disorder diagnosis and using } \\
1 \text { or more substances regularly }\end{array}$ \\
\hline Interventions & $\begin{array}{l}\text { Intervention: adolescent skills, parent management, parent-adolescent negotiation, acute treat- } \\
\text { ment for } 12 \text { weeks followed by } 3 \text { monthly booster sessions }\end{array}$ \\
\hline Control: treatment as usual
\end{tabular}

\section{Conrod 2017}

\section{Trial name or title}

Inter-Venture: A Cluster Randomized Controlled Trial Investigating the Effect of School-Based Personality-Targeted Interventions and Collaborative Youth Mental Health Care

\begin{tabular}{ll}
\hline Methods & Randomised controlled trial \\
\hline Participants & $\begin{array}{l}4000 \text { adolescents aged } 12 \text { to } 17 \text { years recruited from public or private schools offering courses from } \\
\text { grade } 7 \text { to grade } 11\end{array}$ \\
\hline
\end{tabular}

Interventions

Intervention 1: Preventure, Equipe and Inter-Action. Preventure is a personality-targeted intervention conducted using manuals that incorporate psychoeducational, motivational enhancement therapy and cognitive-behavioural components. The Equipe programme is for groups of parents of 13- to 18-year-old adolescents. It uses a coping modelling problem-solving process in which parents are the key players in developing problem-solving strategies and includes group workshops addressing communication, conflict resolution, co-operative transitions, negotiating, house rules, monitoring, consequences for serious problems, and problem-solving. Inter-Action services is an intervention model designed to provide integrated services for youth with significant symptoms of mental health problems, substance misuse, and/or psychosocial difficulties. It may include cognitive-behavioural therapy, motivational interviews, dialectic behavioural therapy, and family interventions provided by multi-disciplinary teams of professionals

Intervention 2: Preventure and Equipe, as described for Intervention 1

Control: treatment as usual

Outcomes

Alcohol and drug problems in adolescents assessed by the DEP-ADO, a self-report measure of age of onset, frequency, and consequences of alcohol and illicit drug use in adolescents

Starting date Actual start date: April 2017


Conrod 2017 (Continued)

Notes ClinicalTrials.gov identifier: NCT03114007

Ford 2015

Trial name or title A Pilot Efficacy and Implementation Study of the Patients, Parents and Professionals Partnering to Improve Adolescent Health (P4) Intervention

\begin{tabular}{|c|c|}
\hline Methods & Randomised controlled trial \\
\hline Participants & Youth aged 14 to 17 years from the CHOP primary care network \\
\hline \multirow[t]{5}{*}{ Interventions } & $\begin{array}{l}\text { Intervention } 1 \text { : sexual health for parents of adolescents aged } 14 \text { to } 15 \text {, psychoeducational work- } \\
\text { book, worksheets, tip sheets, and health coaching sessions about sexual health, STD prevention, } \\
\text { and safe sex practices for teenagers }\end{array}$ \\
\hline & $\begin{array}{l}\text { Intervention 2: alcohol prevention for parents of adolescents aged } 14 \text { to } 15, \text { psychoeducational } \\
\text { workbook, worksheets, tip sheets, and health coaching sessions about alcohol prevention and } \\
\text { safety, underage drinking, and drinking and driving }\end{array}$ \\
\hline & $\begin{array}{l}\text { Control for Interventions } 1 \text { and 2: sexual health and alcohol control group, usual care well-child ap- } \\
\text { pointment }\end{array}$ \\
\hline & $\begin{array}{l}\text { Intervention 3: teen driving for parents of adolescents aged } 16 \text { to } 17 \text {, psychoeducational workbook, } \\
\text { worksheets, videos, tip sheets, and health coaching sessions about how parents can help super- } \\
\text { vise their teens' safe driving practices and can talk with their teen about important safety topics for } \\
\text { teen drivers }\end{array}$ \\
\hline & Control for Intervention 3: teen driving control group, usual care well-child appointment \\
\hline Outcomes & Quality of parent-teen communication about alcohol \\
\hline Starting date & Actual start date: January 2016 \\
\hline Contact information & Carol Ford, Children's Hospital of Philadelphia \\
\hline Notes & ClinicalTrials.gov identifier: NCT02554682 \\
\hline
\end{tabular}

Hops 2012

Trial name or title Early Intervention for Minors in Possession of Alcohol/Drugs: A Feasibility Study

\begin{tabular}{ll}
\hline Methods & Randomised controlled trial \\
\hline Participants & 280 adolescents 13 to 17 years old charged with a drug-related offense \\
\hline Interventions & Intervention 1: Motivational Enhancement Therapy for adolescents, Parenting Wisely for parents \\
& Intervention 2: Motivational Enhancement Therapy for adolescents \\
& Intervention 3: Drug Education for adolescents, Parenting Wisely for parents \\
& Intervention 4: Drug Education for adolescents
\end{tabular}

Outcomes

Adolescent substance use and related problems assessed using the Time Line Follow Back Questionnaire 
Hops 2012 (Continued)

Starting date Actual start date: July 2011

\begin{tabular}{ll}
\hline Contact information & Hyman Hops, Oregon Research Institute \\
\hline
\end{tabular}

Notes

ClinicalTrials.gov identifier: NCT01616212

Kogan 2018

\begin{tabular}{|c|c|}
\hline Trial name or title & Strong African American Families STEPS Project (SAAF-STEPS) \\
\hline Methods & Randomised controlled trial \\
\hline Participants & African American youth aged 11 to 15 \\
\hline \multirow[t]{5}{*}{ Interventions } & $\begin{array}{l}\text { A 4-arm trial, with participants receiving a preadolescent intervention only, a mid-adolescent inte } \\
\text { vention only, both preadolescent and mid-adolescent interventions, or no intervention }\end{array}$ \\
\hline & $\begin{array}{l}\text { Intervention 1: Pre-Adolescent Strong African American Families (SAAF) at age } 11 \text { to } 12 \text { (7-session } \\
\text { family skills training programme) }\end{array}$ \\
\hline & Intervention 2: SAAF Teen delivered at age 14 to 15 (5-session family skills training programme) \\
\hline & Intervention 3: SAAF (age 11 to 12 ) and SAAF-T (age 14 to 15 ) \\
\hline & Control: no intervention \\
\hline
\end{tabular}

Outcomes Alcohol use in past 3 months; youth completing this single item from Monitoring the Future Study, assessing the frequency of alcohol use in the past 3 months. An ordinal response scale ranges from 0 (none) to 6 (30 or more times). Higher response numbers indicate worse outcomes (more alcohol use)

\begin{tabular}{ll}
\hline Starting date & Actual start date: 10 December 2012 \\
\hline Contact information & Steve Kogan, University of Georgia \\
\hline Notes & ClinicalTrials.gov Identifier: NCT03590132 \\
\hline
\end{tabular}

\section{McCart 2017}

\begin{tabular}{|c|c|}
\hline Trial name or title & Experimental Mediation Research Aimed at Enhancing Adolescent Substance Abuse Treatment \\
\hline Methods & Randomised controlled trial \\
\hline Participants & 172 adolescents aged 12 to 17 years with a current substance use disorder \\
\hline Interventions & $\begin{array}{l}\text { Intervention: enhanced Contingency Management }(\mathrm{CM}+) \text {; standard } \mathrm{CM} \text { uses behaviour modifica- } \\
\text { tion and cognitive-behavioural strategies to target adolescent substance use. The provider con- } \\
\text { ducts Antecedent-Behavior-Consequence assessments of the youth's substance use with both } \\
\text { youth and caregiver. Results of the } \mathrm{ABC} \text { assessment inform self-management planning and drug } \\
\text { refusal skills training. A contract is established with the family, which provides the youth with re- } \\
\text { wards/privileges for negative drug and alcohol tests, and disincentives (e.g. extra chores) for posi- } \\
\text { tive tests. These steps are repeated until continued abstinence is achieved. CM typically is } 12 \text { to } 16 \\
\text { weeks in duration }\end{array}$ \\
\hline
\end{tabular}


In addition to standard CM, CM+ includes the behavioural assessment and teaching system from Parent Management Training Oregon (PMTO). PMTO includes daily parent reports on youth behaviour, anti-coercive problem-solving, and structured learning, as well as in vivo practice of new parenting techniques

Control: standard Contingency Management (CM), as described for standard CM

\begin{tabular}{ll}
\hline Outcomes & $\begin{array}{l}\text { Youth Urine Drug/Alcohol Screening for alcohol metabolites (ethyl glucuronide, ethyl sulfate) } \\
\text { Frequency of substance use and substance-related problems as measured by the Global Appraisal } \\
\text { of Individual Needs (youth-report) }\end{array}$ \\
\hline Starting date & Anticipated start date: March 2018 \\
\hline Contact information & Michael McCart, Oregon Social Learning Center; MikeM@oslc.org \\
\hline Notes & ClinicalTrials.gov identifier: NCT03249350 \\
\hline
\end{tabular}

\section{Mello 2016}

\begin{tabular}{|c|c|}
\hline Trial name or title & An e-Parenting Skills Intervention to Decrease Injured Adolescents' Alcohol Use \\
\hline Methods & Randomised controlled trial \\
\hline Participants & $\begin{array}{l}\text { Adolescents aged } 12 \text { to } 17 \text { admitted to the trauma service with a positive screening for alcohol or } \\
\text { drug use }\end{array}$ \\
\hline \multirow[t]{2}{*}{ Interventions } & $\begin{array}{l}\text { Intervention: standard care plus e-parenting group; standard care is a brief intervention provided } \\
\text { by a trauma centre social worker. The parent receives an e-parenting skills intervention consisting } \\
\text { of an online parent training programme - Parenting Wisely (PW), plus text messaging and a web- } \\
\text { based message board }\end{array}$ \\
\hline & Control: standard trauma centre care as described for the intervention group \\
\hline \multirow[t]{2}{*}{ Outcomes } & $\begin{array}{l}\text { Alcohol use will be measured by the Adolescent Drug Questionnaire (numbers of drinking days and } \\
\text { heavy drinking days in the past } 3 \text { months) }\end{array}$ \\
\hline & $\begin{array}{l}\text { Alcohol- and drug-related problems will be measured by } 9 \text { questions from the Add Health Survey } \\
\text { (frequency of psychosocial and health consequences associated with alcohol and drug use over the } \\
\text { prior 3-month period) }\end{array}$ \\
\hline Starting date & Actual start date: September 2015 \\
\hline Contact information & Michael Mello, Injury Prevention Center, Rhode Island Hospital \\
\hline Notes & ClinicalTrials.gov identifier: NCT02718508 \\
\hline
\end{tabular}

\section{Miller 2009}

\begin{tabular}{ll}
\hline Trial name or title & Adolescent Family-Based Alcohol Prevention \\
\hline Methods & Randomised controlled trial \\
\hline
\end{tabular}


Miller 2009 (Continued)

Participants

Families who were members of one of 4 Kaiser Permanente medical centres in Northern California (Oakland, Vallejo, San Francisco, and Walnut Creek) at the time the sample was drawn, who had an 11- or 12-year-old child

Interventions

All groups received an informational pamphlet about youth alcohol and other drug use

Intervention 1: Family Matters prevention programme with 4 booklets for families to do at home with their child

Intervention 2: Strengthening Families Program (SFP) prevention programme with 7 weekly group sessions for parent and child

Half of the families were able to choose (rather than be assigned) either Intervention 1 or 2

Control: no programme other than the information pamphlet described above

\begin{tabular}{ll}
\hline Outcomes & Youth alcohol and other drug use \\
\hline Starting date & Actual start date: April 2005 \\
\hline Contact information & Brenda Miller, Pacific Institute for Research and Evaluation \\
\hline Notes & ClinicalTrails.gov identifier: NCT00858065 \\
\hline
\end{tabular}

Miller 2018

Trial name or title Family Based Prevention of Alcohol and Risky Sex for Older Teens

\begin{tabular}{ll}
\hline Methods & Randomised controlled trial \\
\hline Participants & Parents and 16- or 17-year-old teens \\
\hline Interventions & $\begin{array}{l}\text { Intervention: Smart Choices } 4 \text { Teens - a web-based intervention consisting of } 3 \text { main components } \\
\text { (Communication, Alcohol, Relationships) provided to both parents and teens and completed by } \\
\text { parents and teens individually. At the end of each component, discussion guidelines were given to } \\
\text { promote communications and to offer skills-building practices between parent and teen regarding } \\
\text { the component topic. Both parents and teens were required to complete the component and dis- } \\
\text { cussion before moving to the next component } \\
\text { Control: no intervention }\end{array}$ \\
\hline
\end{tabular}

Outcomes

Change in alcohol use over time [ Time Frame: baseline, 6, 12, and 18 months ] Change in prevalence of use ever, past 6 months, past 30 days

Change in parent-teen (general) communications over time [ Time Frame: baseline, 6, 12, and 18 months ] Changes in the quality of general communication from baseline to post-intervention time periods

Change in parent-teen communications regarding teen alcohol use over time [ Time Frame: baseline, 6, 12, and 18 months ] Count of topics covered (consequences of drinking, expectations for teen alcohol use, refusal strategies, rules) from baseline to post-intervention time periods

Change in parent-teen discussions about safe drinking for teens over time [ Time Frame: baseline, 6,12 , and 18 months ] Changes in parental approval of teen drinking in moderation or under certain conditions, from baseline to post-intervention time periods 
Miller 2018 (Continued)

Change in parent-teen communications about social host laws over time [ Time Frame: baseline, 6,12 , and 18 months ] Changes in prevalence/frequency of communications about laws from baseline to post-intervention time periods

\begin{tabular}{ll}
\hline Starting date & Actual start date: April 2015 \\
\hline Contact information & Brenda Miller, Pacific Institute for Research and Evaluation \\
\hline Notes & ClinicalTrials.gov identifier: NCT03521115 \\
\hline
\end{tabular}

Rowe 2010

\begin{tabular}{ll}
\hline Trial name or title & Family Intervention for Teen Drinking and Alcohol-Related Crises in the ER \\
\hline Methods & Randomised controlled trial \\
\hline Participants & Adolescents aged 12 to 18 years who present to the ER or trauma unit with alcohol problems \\
\hline Interventions & $\begin{array}{l}\text { Intervention 1: Multi-dimensional Family Therapy, an outpatient family-based treatment including } \\
\text { two } 60 \text { - to 90-minute sessions per week for } 3 \text { months plus homework and phone contacts as need- } \\
\text { ed }\end{array}$ \\
$\begin{array}{l}\text { Intervention 2: Family Motivational Interviewing, with youth and parents receiving } 2 \text { sessions in } \\
\text { their home within } 72 \text { hours of the ER incident and with youth also provided standard care as de- } \\
\text { scribed for the control group } \\
\text { Control: standard care, two 90-minute group sessions per week for } 3 \text { months }\end{array}$ \\
\hline Outcomes & Alcohol use/abstinence \\
\hline Starting date & Actual start date: November 2010 \\
\hline Contact information & Cynthia Rowe, University of Miami \\
\hline Notes & ClinicalTrials.gov identifier: NCT01229748 \\
\hline
\end{tabular}

\section{Segrott 2014}

Trial name or title ing Families Programme 10-14 UK (SFP 10-14 UK)
Preventing Substance Misuse: Study Protocol for a Randomised Controlled Trial of the Strengthen- 
Segrott 2014 (Continued)

Control: usual care with full access to existing services. No defined programme of usual care

Outcomes

Primary outcomes concerned with youth alcohol use prevalence and drunkenness prevalence; secondary outcomes concerned with long-term alcohol/tobacco/substance behaviours; tertiary outcomes concerned with health and family well-being, and with substance use initiation

\begin{tabular}{ll}
\hline Starting date & NR \\
\hline Contact information & Jeremy Segrott \\
\hline Notes & Current Controlled Trials identifier: ISRCTN63550893. \\
\hline
\end{tabular}

\section{Sheidow 2017}

$\begin{array}{ll}\text { Trial name or title } & \begin{array}{l}\text { Improving Access to Substance Abuse Evidence-Based Practices for Youth in the Justice System: } \\ \text { Strategies Used by JPOs }\end{array}\end{array}$

Methods Randomised controlled trial

Participants 504 youth aged 12 to 17 years with a newly opened probation case

Interventions

Intervention: Contingency Management (CM): CM uses behaviour modification and cognitive-behavioural strategies to target adolescent substance use. The provider (juvenile probation officer) conducts Antecedent-Behavior-Consequence assessments of the youth's substance use with both youth and caregiver. Results of the $A B C$ assessment inform self-management planning and drug refusal skills training. A contract is established with the family, which provides the youth with rewards/privileges for negative drug and alcohol tests, and disincentives (e.g. extra chores) for positive tests. These steps are repeated until continued abstinence is achieved. CM typically is 12 to 16 weeks in duration

Control: standard services that a young person would receive while under probation supervision in the state of Oregon

Youth Urine Drug/Alcohol Screening for alcohol metabolites (ethyl glucuronide, ethyl sulfate)
Frequency of substance use and substance-related problems as measured by the Global Appraisal
of Individual Needs (youth-report)

Starting date Anticipated start date: October 2017

\begin{tabular}{ll}
\hline Contact information & Ashli Sheidow, Oregon Social Learning Center; AshliS@oslc.org \\
\hline Notes & ClinicalTrials.gov identifier: NCT03015805 \\
\hline
\end{tabular}

Spirito 2017b

\begin{tabular}{ll}
\hline Trial name or title & Computer-Assisted Brief Intervention Protocol for Marijuana-Using Juvenile Offenders \\
\hline Methods & Randomised controlled trial \\
\hline Participants & 80 adolescents aged 14 to 17 with a positive result for marijuana use on intake \\
\hline Interventions & $\begin{array}{l}\text { Intervention: computer counselling, a computer-assisted motivational intervention called e-toke } \\
\text { plus an online parenting programme called Parenting Wisely }\end{array}$
\end{tabular}


Spirito 2017b (Continued)

Contol: standard care, referral to counselling for substance use

Outcomes Total number of alcohol use days assessed by the Time Line Follow Back interview

Alcohol use problems assessed by items from the Add Health longitudinal study

\begin{tabular}{ll}
\hline Starting date & Estimated start date: October 2017 \\
\hline Contact information & Anthony Spirito, Brown University; anthony_spirito@brown.edu \\
\hline Notes & ClinicalTrials.gov identifier: NCT03107117 \\
\hline
\end{tabular}

Stanton 2007

\begin{tabular}{ll}
\hline Trial name or title & Family and Group Therapies for Adolescent Alcohol Abuse \\
\hline Methods & Randomised controlled trial \\
\hline Participants & Adolescents aged 13 to 17 years with a DSM-IV diagnosis of alcohol abuse or alcohol dependence \\
\hline Interventions & Intervention 1: adolescent group therapy \\
& Intervention 2: transitional family therapy \\
\hline Outcomes & Alcohol use \\
\hline Starting date & Actual start date: July 1999 \\
\hline Contact information & Morris Stanton, The Morton Center \\
\hline Notes & ClinicalTrials.gov identifier: NCT00484367 \\
\hline
\end{tabular}

Whitbeck 2016

\begin{tabular}{|c|c|}
\hline Trial name or title & A Randomized Control Trial (RCT) of a Family-Centered Ojibwe Substance Abuse Prevention \\
\hline Methods & Randomised controlled trial \\
\hline Participants & 1500 youth aged 8 to 10 years from Anishinaabe communities \\
\hline Interventions & $\begin{array}{l}\text { Intervention: Bii-Zin-Da-De-Dah (Listening to One Another) Intervention. Bii-Zin-Da-De-Dah (Listen- } \\
\text { ing to One Another) is an American Indian adaptation of the lowa Strengthening Families Program } \\
\text { (now called the Strengthening Families Program). The programme is } 14 \text { weeks in duration. The first } \\
4 \text { weeks of the programme is oriented towards Anishinaabe cultural traditions and the traditional } \\
\text { Anishinaabe family. Weeks } 5 \text { through } 8 \text { focus on identifying feelings and learning how to manage } \\
\text { negative feelings such as anger and sadness in positive ways. The last } 6 \text { weeks of the programme } \\
\text { focuses on outside influences and ways to build positive support systems }\end{array}$ \\
\hline & Control: no intervention \\
\hline
\end{tabular}

Outcomes Frequency of alcohol use

Starting date Actual start date: January 2017


Whitbeck 2016 (Continued)

Contact information Leslie Whitbeck, University of Nebraska Lincoln

Notes ClinicalTrials.gov identifier: NCT02700035

ER: emergency room.

NR: not reported.

STD: sexually transmitted disease.

\section{DATA AND ANALYSES}

\section{Comparison 1. Any parent/family intervention vs no intervention/standard care}

\begin{tabular}{|c|c|c|c|c|}
\hline Outcome or subgroup title & $\begin{array}{l}\text { No. of } \\
\text { studies }\end{array}$ & $\begin{array}{l}\text { No. of } \\
\text { partici- } \\
\text { pants }\end{array}$ & Statistical method & Effect size \\
\hline 1 Alcohol use_Prevalence & 12 & 7490 & Std. Mean Difference (Random, 95\% Cl) & $0.00[-0.08,0.08]$ \\
\hline 2 Alcohol use_Frequency & 8 & 1835 & Std. Mean Difference (Random, 95\% Cl) & $-0.31[-0.83,0.21]$ \\
\hline 3 Alcohol use_Volume & 5 & 1825 & Std. Mean Difference (Random, 95\% Cl) & $-0.14[-0.27,0.00]$ \\
\hline $\begin{array}{l}4 \text { Sensitivity_RoB_Alcohol } \\
\text { use_Prevalence }\end{array}$ & 5 & 1733 & Std. Mean Difference (Random, 95\% Cl) & $-0.12[-0.35,0.11]$ \\
\hline $\begin{array}{l}5 \text { Subgroup_universal_Alcohol } \\
\text { use_Prevalence }\end{array}$ & 10 & 7133 & Std. Mean Difference (Random, 95\% Cl) & $0.02[-0.06,0.11]$ \\
\hline $\begin{array}{l}6 \text { Subgroup_selective/indicat- } \\
\text { ed_Alcohol use_Prevalence }\end{array}$ & 2 & 357 & Std. Mean Difference (Random, 95\% Cl) & $-0.16[-0.36,0.05]$ \\
\hline $\begin{array}{l}7 \text { Subgroup_low intensity_Alco- } \\
\text { hol use_Prevalence }\end{array}$ & 6 & & Std. Mean Difference (Random, 95\% Cl) & $-0.01[-0.10,0.08]$ \\
\hline $\begin{array}{l}8 \text { Subgroup_>12 months_Alcohol } \\
\text { use_Prevalence }\end{array}$ & 11 & & Std. Mean Difference (Random, 95\% Cl) & $0.00[-0.08,0.09]$ \\
\hline $\begin{array}{l}9 \text { Subgroup_ethnicity minori- } \\
\text { ty_Alcohol use_Prevalence }\end{array}$ & 3 & 325 & Std. Mean Difference (Random, 95\% Cl) & $-0.20[-0.42,0.02]$ \\
\hline $\begin{array}{l}10 \text { Subgroup_ethnicity majori- } \\
\text { ty/caucasian_Alcohol use_Preva- } \\
\text { lence }\end{array}$ & 9 & & Std. Mean Difference (Random, 95\% Cl) & $0.03[-0.06,0.11]$ \\
\hline $\begin{array}{l}11 \text { Subgroup_no weekly or heavy } \\
\text { drinking_Alcohol use_Prevalence }\end{array}$ & 9 & & Std. Mean Difference (Random, 95\% Cl) & $0.01[-0.12,0.13]$ \\
\hline $\begin{array}{l}12 \text { Sensitivity_RoB_Alcohol } \\
\text { use_Frequency }\end{array}$ & 5 & 1488 & Std. Mean Difference (Random, 95\% Cl) & $0.09[-0.24,0.43]$ \\
\hline $\begin{array}{l}13 \text { Subgroup_universal_Alcohol } \\
\text { use_Frequency }\end{array}$ & 3 & 1090 & Std. Mean Difference (Random, 95\% Cl) & $0.18[-0.40,0.75]$ \\
\hline
\end{tabular}




\begin{tabular}{|c|c|c|c|c|}
\hline Outcome or subgroup title & $\begin{array}{l}\text { No. of } \\
\text { studies }\end{array}$ & $\begin{array}{l}\text { No. of } \\
\text { partici- } \\
\text { pants }\end{array}$ & Statistical method & Effect size \\
\hline $\begin{array}{l}14 \text { Subgroup_selective/indicat- } \\
\text { ed_Alcohol use_Frequency }\end{array}$ & 5 & 745 & Std. Mean Difference (Random, 95\% Cl) & $-0.65[-1.64,0.33]$ \\
\hline $\begin{array}{l}15 \text { Subgroup_>12 months_Alco- } \\
\text { hol use_Frequency }\end{array}$ & 8 & 1835 & Std. Mean Difference (Random, 95\% Cl) & $-0.31[-0.83,0.21]$ \\
\hline $\begin{array}{l}16 \text { Subgroup_ethnicity minori- } \\
\text { ty_Alcohol use_Frequency }\end{array}$ & 3 & 1037 & Std. Mean Difference (Random, 95\% Cl) & $-1.19[-2.83,0.46]$ \\
\hline $\begin{array}{l}17 \text { Subgroup_ethnicity majori- } \\
\text { ty/caucasian_Alcohol use_Fre- } \\
\text { quency }\end{array}$ & 5 & 798 & Std. Mean Difference (Random, 95\% Cl) & $0.15[-0.13,0.43]$ \\
\hline $\begin{array}{l}18 \text { Subgroup_no binge_Alcohol } \\
\text { use_Frequency }\end{array}$ & 7 & 1519 & Std. Mean Difference (Random, 95\% Cl) & $-0.37[-1.01,0.27]$ \\
\hline $\begin{array}{l}19 \text { Sensitivity_RoB_Alcohol } \\
\text { use_Volume }\end{array}$ & 4 & 1397 & Std. Mean Difference (Random, 95\% Cl) & $-0.15[-0.32,0.03]$ \\
\hline $\begin{array}{l}20 \text { Subgroup_universal_Alcohol } \\
\text { use_Volume }\end{array}$ & 3 & 1481 & Std. Mean Difference (Random, 95\% Cl) & $-0.21[-0.32,-0.10]$ \\
\hline $\begin{array}{l}21 \text { Subgroup_selective/indicat- } \\
\text { ed_Alcohol use_Volume }\end{array}$ & 2 & 344 & Std. Mean Difference (Random, 95\% Cl) & $0.06[-0.15,0.27]$ \\
\hline $\begin{array}{l}22 \text { Subgroup_>12 months_Alco- } \\
\text { hol use_Volume }\end{array}$ & 3 & 988 & Std. Mean Difference (Random, 95\% Cl) & $-0.16[-0.35,0.02]$ \\
\hline $\begin{array}{l}23 \text { Subgroup_ethnicity_minori- } \\
\text { ty_Alcohol use_Volume }\end{array}$ & 3 & 1081 & Std. Mean Difference (Random, 95\% Cl) & $-0.24[-0.36,-0.12]$ \\
\hline $\begin{array}{l}24 \text { Subgroup_ethnicity majori- } \\
\text { ty/caucasian_Alcohol use_Vol- } \\
\text { ume }\end{array}$ & 2 & 744 & Std. Mean Difference (Random, 95\% Cl) & $-0.01[-0.17,0.15]$ \\
\hline $\begin{array}{l}25 \text { Subgroup_female only_Alco- } \\
\text { hol use_Volume }\end{array}$ & 2 & 1053 & Std. Mean Difference (Random, 95\% Cl) & $-0.25[-0.37,-0.13]$ \\
\hline
\end{tabular}

\section{Analysis 1.1. Comparison 1 Any parent/family intervention vs no intervention/standard care, Outcome 1 Alcohol use_Prevalence.}

\begin{tabular}{|c|c|c|c|c|c|c|}
\hline \multirow{3}{*}{$\begin{array}{l}\text { Study or subgroup } \\
\text { Baldus } 2016\end{array}$} & \multirow{3}{*}{$\begin{array}{l}\text { Any fam- } \\
\text { ily inter- } \\
\text { vention } \\
\text { N } \\
132\end{array}$} & \multirow{3}{*}{$\begin{array}{l}\begin{array}{c}\text { Stan- } \\
\text { dard care }\end{array} \\
\begin{array}{r}\text { N } \\
125\end{array}\end{array}$} & \multirow{3}{*}{$\begin{array}{c}\begin{array}{c}\text { Std. Mean } \\
\text { Difference }\end{array} \\
\text { (SE) } \\
-0.1(0.124)\end{array}$} & \multirow[t]{2}{*}{$\begin{array}{l}\text { Std. Mean Difference } \\
\text { IV, Random, 95\% CI }\end{array}$} & \multirow{3}{*}{$\begin{array}{r}\text { Weight } \\
6.9 \%\end{array}$} & \multirow{3}{*}{$\begin{array}{l}\text { Std. Mean Difference } \\
\text { IV, Random, 95\% Cl } \\
-0.11[-0.35,0.13]\end{array}$} \\
\hline & & & & & & \\
\hline & & & & \begin{tabular}{l|l}
+ & \\
\end{tabular} & & \\
\hline Bauman 2002 & 531 & 604 & $0.2(0.06)$ & $\rightarrow$ & $13.18 \%$ & $0.16[0.04,0.28]$ \\
\hline Bodin 2011 & 893 & 859 & $-0(0.048)$ & $\rightarrow$ & $14.61 \%$ & $-0.01[-0.1,0.09]$ \\
\hline Brody 2006 & 44 & 34 & $-0.5(0.229)$ & & $2.74 \%$ & $-0.47[-0.92,-0.02]$ \\
\hline Catalano 1999 & 57 & 43 & $-0.3(0.203)$ & - & $3.36 \%$ & $-0.29[-0.69,0.11]$ \\
\hline
\end{tabular}




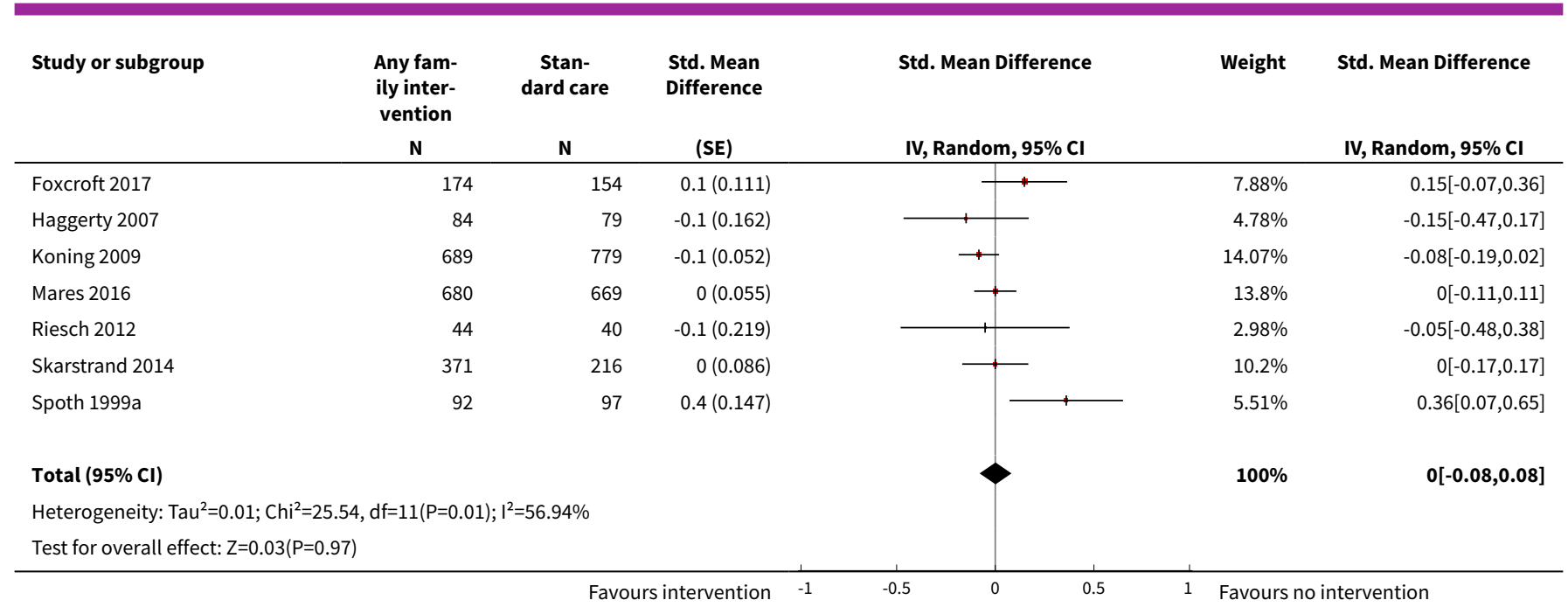

Analysis 1.2. Comparison 1 Any parent/family intervention vs no intervention/standard care, Outcome 2 Alcohol use_Frequency.

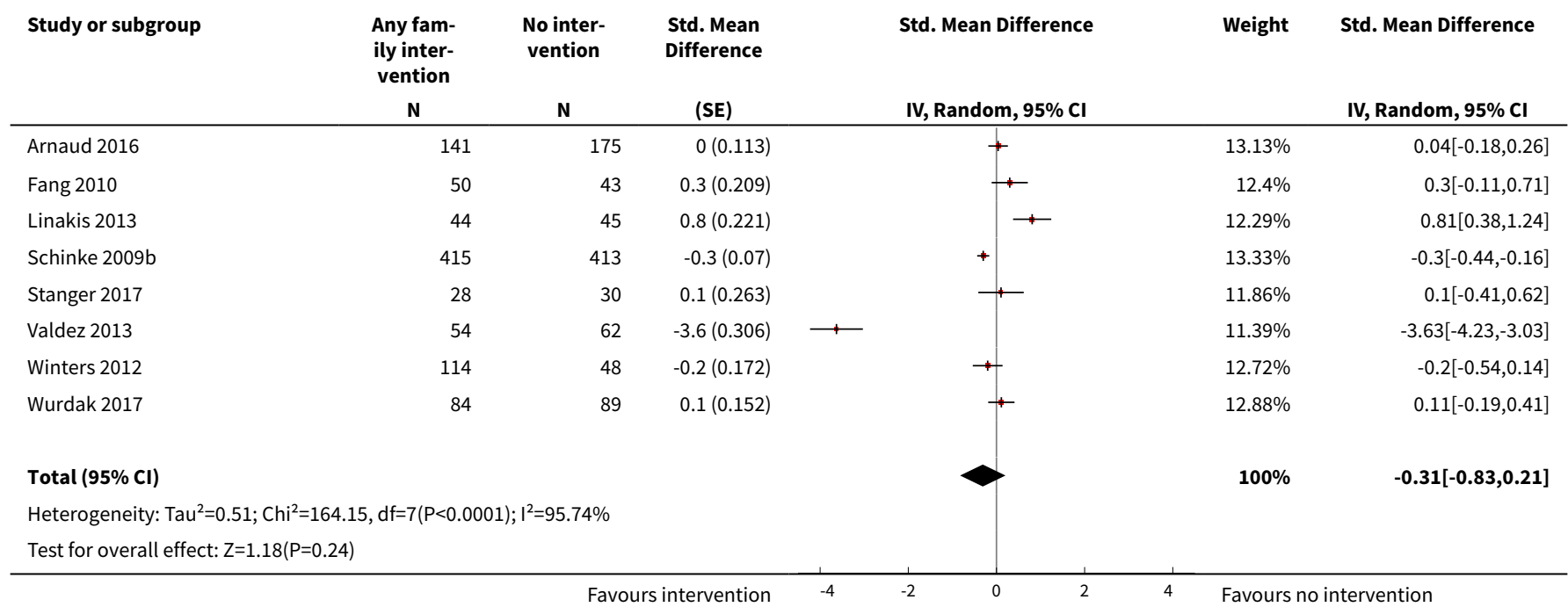

Analysis 1.3. Comparison 1 Any parent/family intervention vs

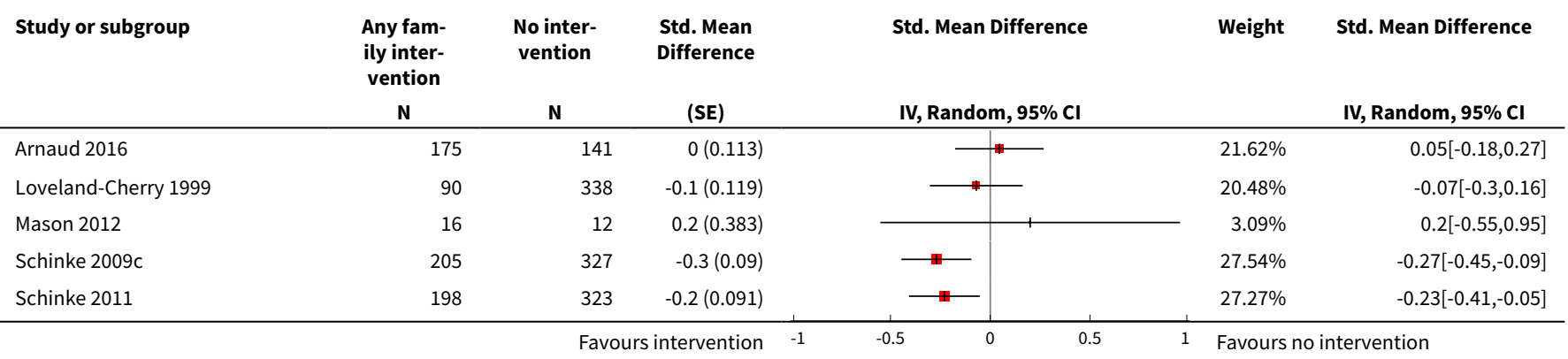




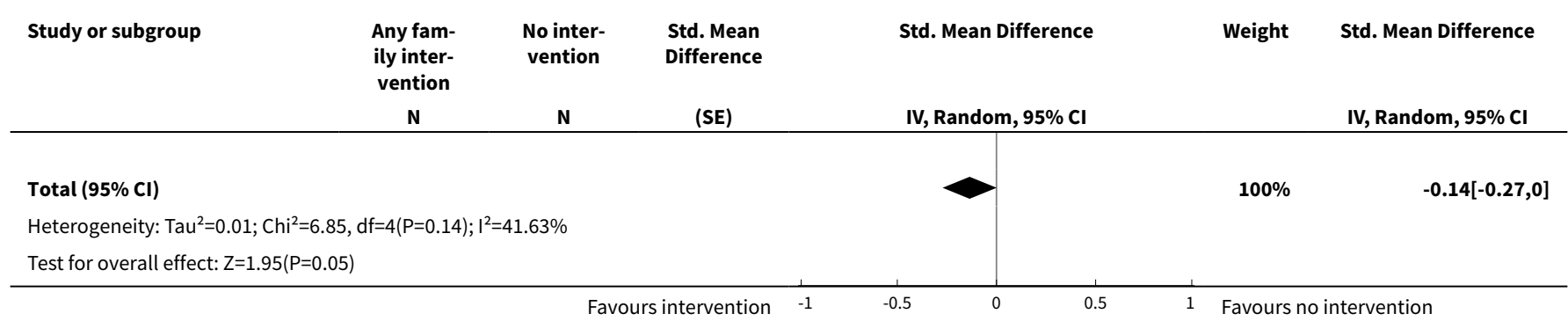

Analysis 1.4. Comparison 1 Any parent/family intervention vs no intervention/ standard care, Outcome 4 Sensitivity_RoB_Alcohol use_Prevalence.

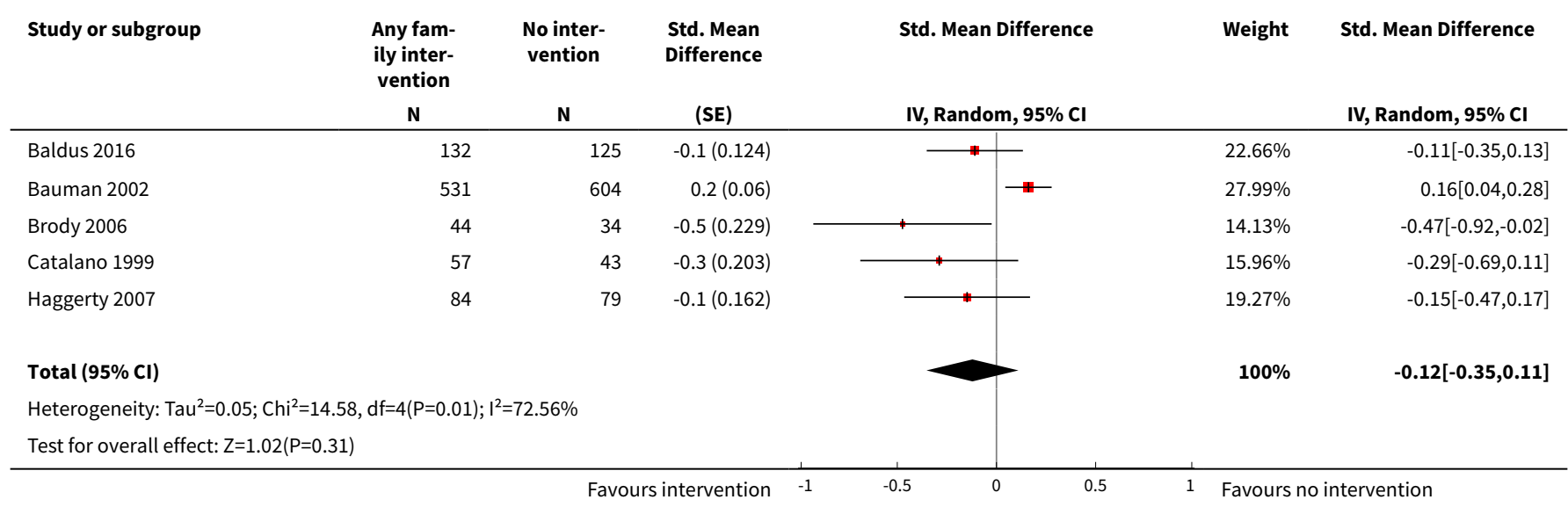

Analysis 1.5. Comparison 1 Any parent/family intervention vs no intervention/ standard care, Outcome 5 Subgroup_universal_Alcohol use_Prevalence.

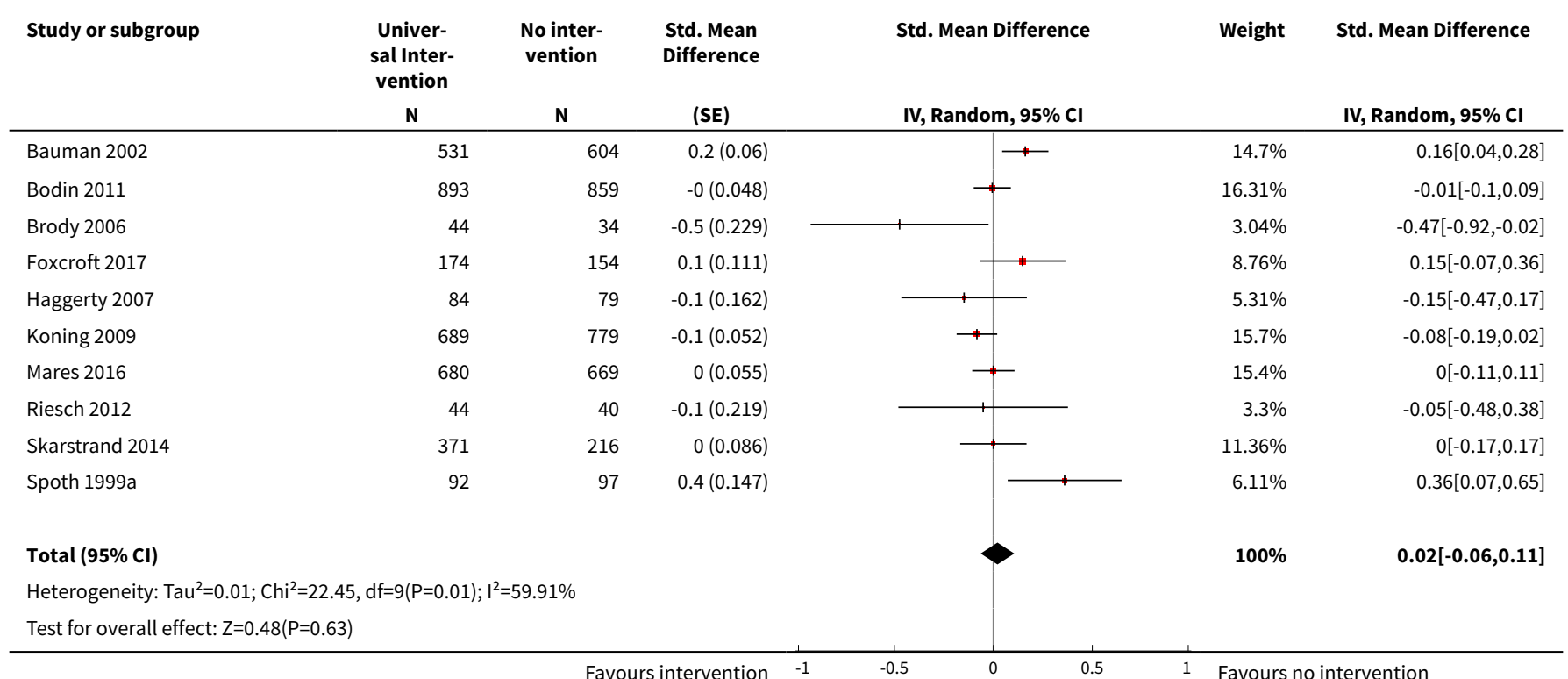


Analysis 1.6. Comparison 1 Any parent/family intervention vs no intervention/ standard care, Outcome 6 Subgroup_selective/indicated_Alcohol use_Prevalence.

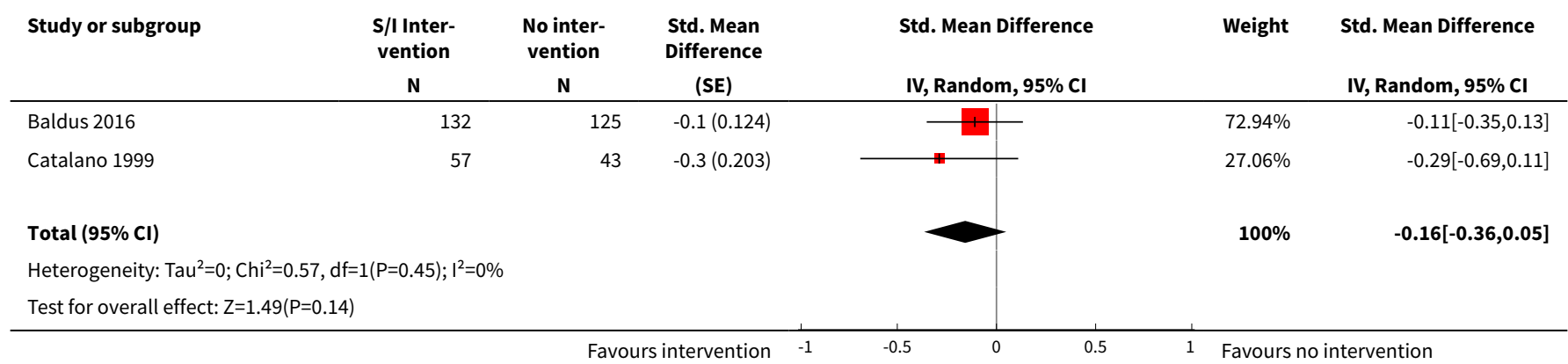

Analysis 1.7. Comparison 1 Any parent/family intervention vs no intervention/ standard care, Outcome 7 Subgroup_low intensity_Alcohol use_Prevalence.

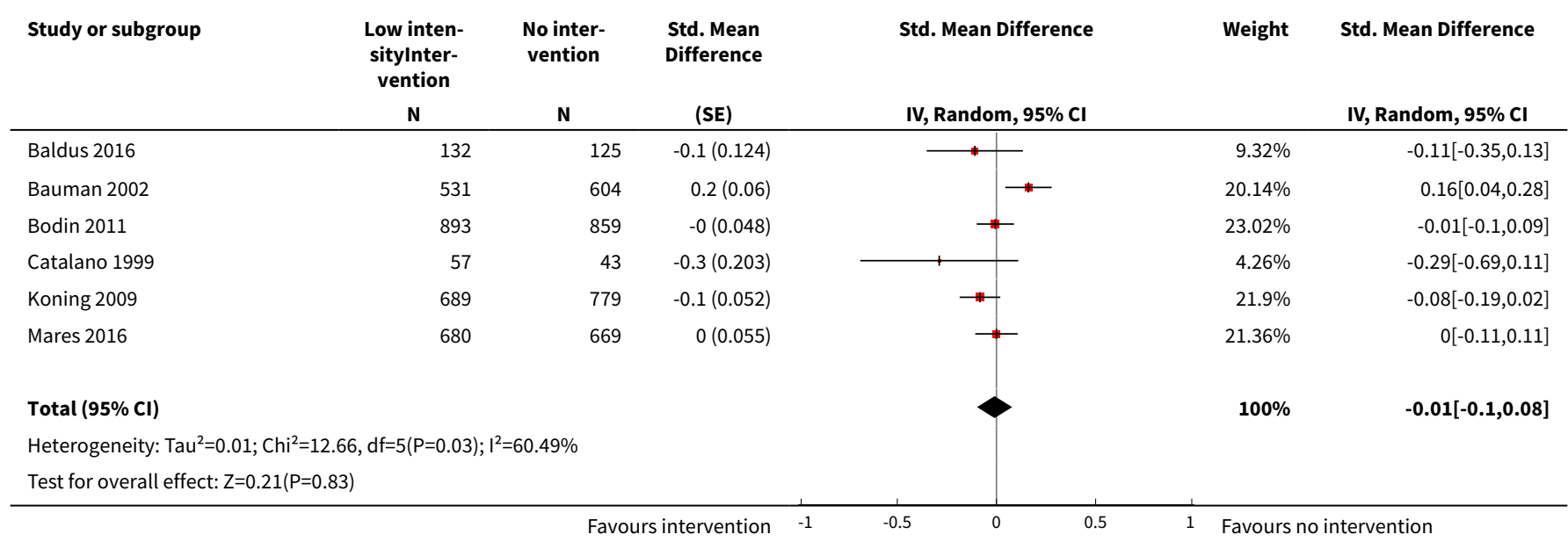

Analysis 1.8. Comparison 1 Any parent/family intervention vs no intervention/ standard care, Outcome 8 Subgroup_>12 months_Alcohol use_Prevalence.

\begin{tabular}{|c|c|c|c|c|c|c|}
\hline \multirow{3}{*}{$\begin{array}{l}\text { Study or subgroup } \\
\text { Baldus } 2016\end{array}$} & \multirow{3}{*}{$\begin{array}{l}\begin{array}{l}\text { Any fam- } \\
\text { ily Inter- } \\
\text { vention } \\
\text { N }\end{array} \\
132\end{array}$} & \multirow{3}{*}{$\begin{array}{l}\begin{array}{l}\text { No inter- } \\
\text { vention }\end{array} \\
\qquad \begin{array}{r}\text { N } \\
125\end{array}\end{array}$} & \multirow{3}{*}{$\begin{array}{c}\begin{array}{c}\text { Std. Mean } \\
\text { Difference }\end{array} \\
\text { (SE) } \\
-0.1(0.124)\end{array}$} & \multirow[t]{2}{*}{$\begin{array}{l}\text { Std. Mean Difference } \\
\text { IV, Random, } 95 \% \mathrm{Cl}\end{array}$} & \multirow{3}{*}{$\begin{array}{r}\text { Weight } \\
7.23 \%\end{array}$} & \multirow{3}{*}{$\begin{array}{l}\text { Std. Mean Difference } \\
\text { IV, Random, 95\% Cl } \\
-0.11[-0.35,0.13]\end{array}$} \\
\hline & & & & & & \\
\hline & & & & $\longrightarrow$ & & \\
\hline Bauman 2002 & 531 & 604 & $0.2(0.06)$ & $\longrightarrow$ & $13.45 \%$ & $0.16[0.04,0.28]$ \\
\hline Bodin 2011 & 893 & 859 & $-0(0.048)$ & $\rightarrow$ & $14.83 \%$ & $-0.01[-0.1,0.09]$ \\
\hline Brody 2006 & 44 & 34 & $-0.5(0.229)$ & - & $2.93 \%$ & $-0.47[-0.92,-0.02]$ \\
\hline Catalano 1999 & 57 & 43 & $-0.3(0.203)$ & - & $3.58 \%$ & $-0.29[-0.69,0.11]$ \\
\hline Foxcroft 2017 & 174 & 154 & $0.1(0.111)$ & + & $8.22 \%$ & $0.15[-0.07,0.36]$ \\
\hline Haggerty 2007 & 84 & 79 & $-0.1(0.162)$ & 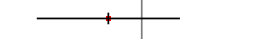 & $5.06 \%$ & $-0.15[-0.47,0.17]$ \\
\hline Koning 2009 & 689 & 779 & $-0.1(0.052)$ & $\rightarrow$ & $14.31 \%$ & $-0.08[-0.19,0.02]$ \\
\hline Mares 2016 & 680 & 669 & $0(0.055)$ & + & $14.05 \%$ & $0[-0.11,0.11]$ \\
\hline
\end{tabular}




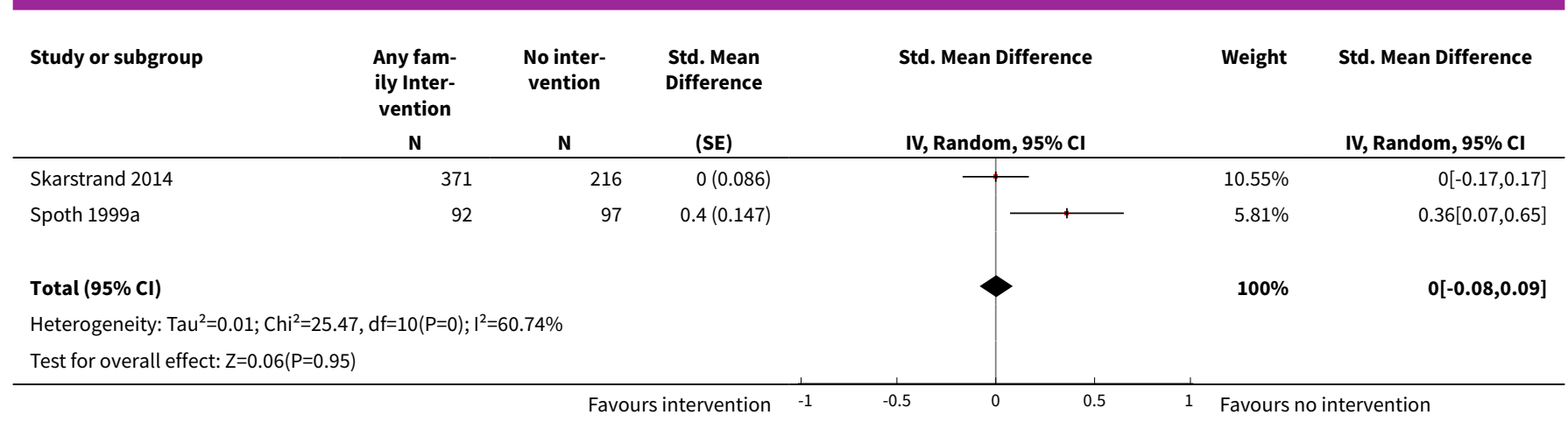

Analysis 1.9. Comparison 1 Any parent/family intervention vs no intervention/ standard care, Outcome 9 Subgroup_ethnicity minority_Alcohol use_Prevalence.

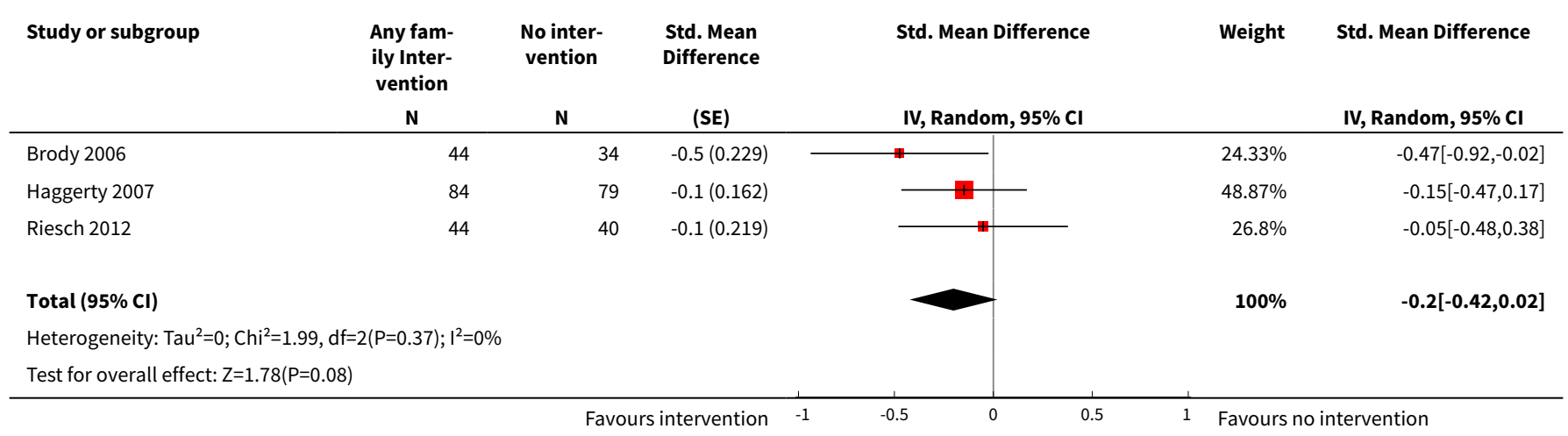

\section{Analysis 1.10. Comparison 1 Any parent/family intervention vs no intervention/standard care, Outcome 10 Subgroup_ethnicity majority/caucasian_Alcohol use_Prevalence.}

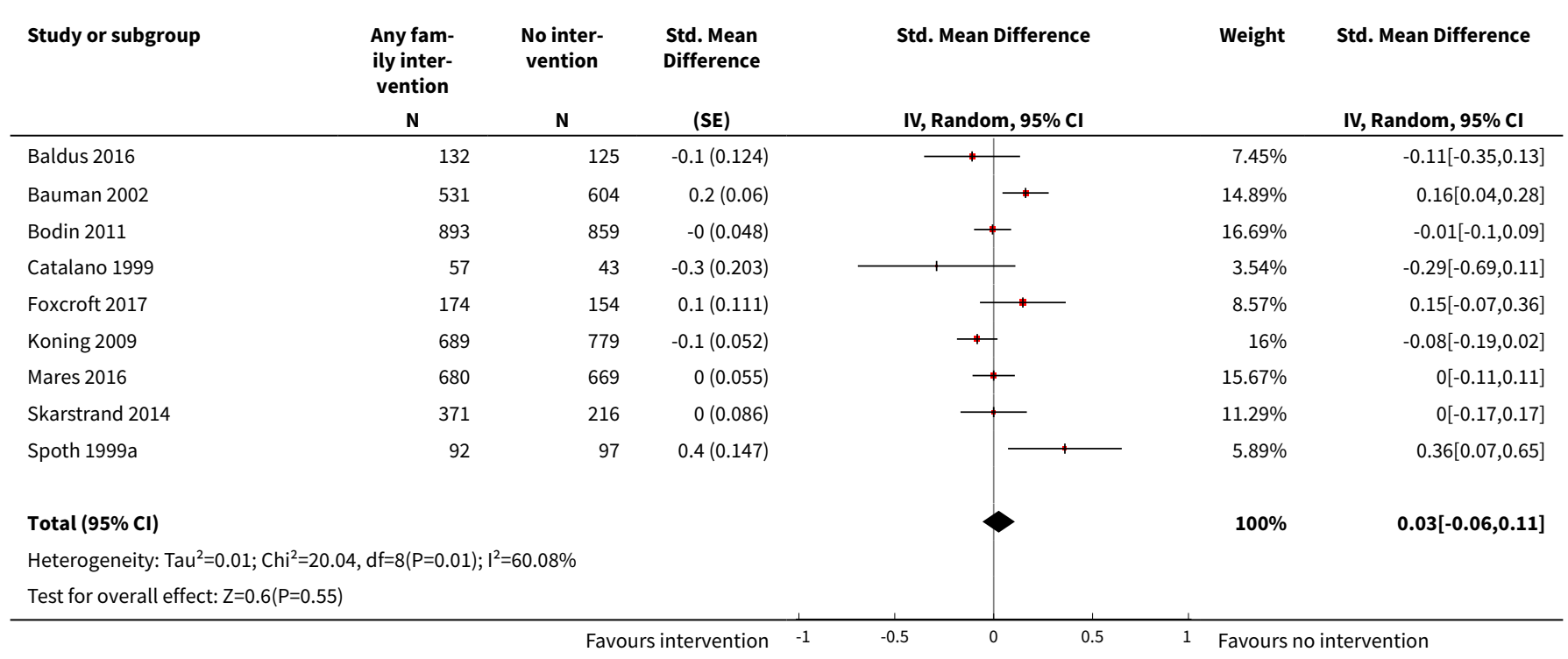


Analysis 1.11. Comparison 1 Any parent/family intervention vs no intervention/standard care, Outcome 11 Subgroup_no weekly or heavy drinking_Alcohol use_Prevalence.

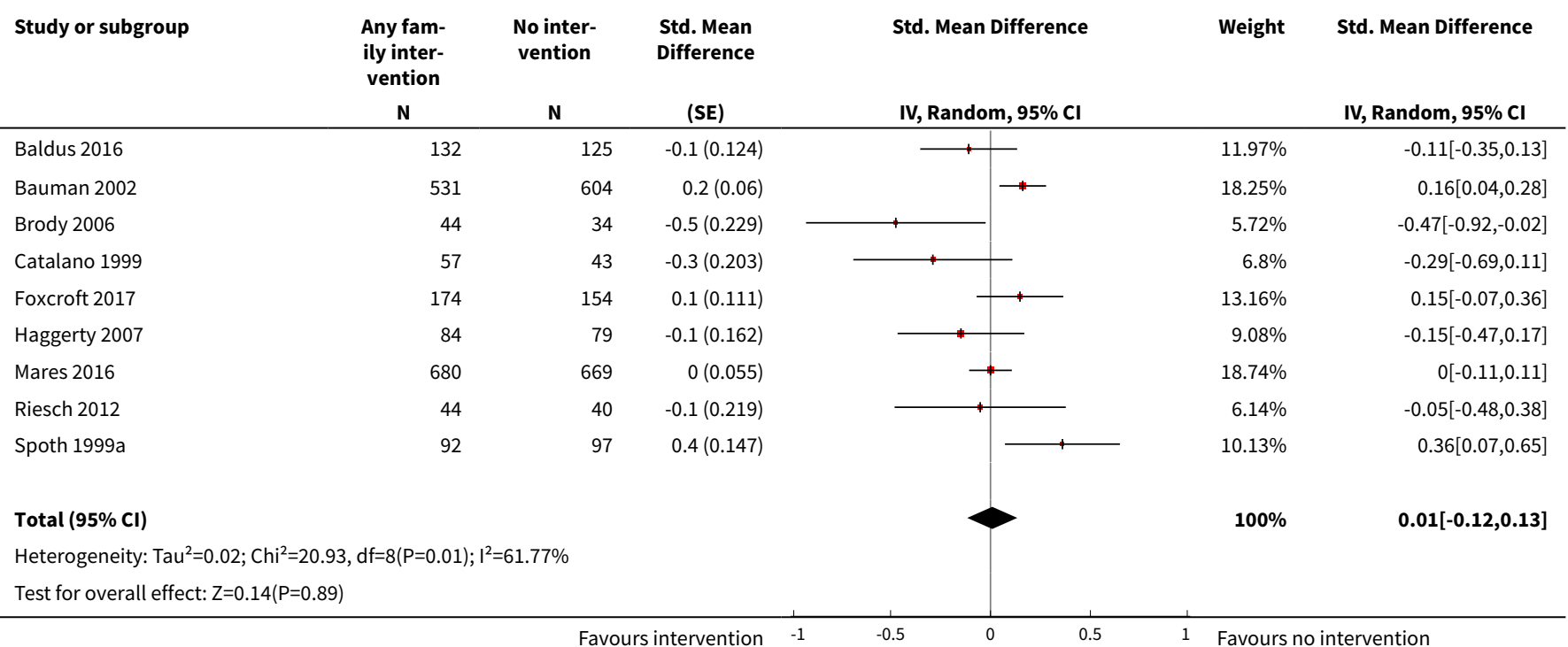

Analysis 1.12. Comparison 1 Any parent/family intervention vs no intervention/ standard care, Outcome 12 Sensitivity_RoB_Alcohol use_Frequency.

\begin{tabular}{|c|c|c|c|c|c|c|}
\hline Study or subgroup & $\begin{array}{l}\text { Any fam- } \\
\text { ily inter- } \\
\text { vention } \\
\mathbf{N}\end{array}$ & $\begin{array}{l}\text { No inter- } \\
\text { vention }\end{array}$ & $\begin{array}{l}\text { Std. Mean } \\
\text { Difference } \\
\text { (SE) }\end{array}$ & Std. Mean Difference & Weight & Std. Mean Difference \\
\hline Arnaud 2016 & 141 & 175 & $0(0.113)$ & + & $21.98 \%$ & $0.04[-0.18,0.26]$ \\
\hline Fang 2010 & 50 & 43 & $0.3(0.209)$ & & $17.84 \%$ & $0.3[-0.11,0.71]$ \\
\hline Linakis 2013 & 44 & 45 & $0.8(0.221)$ & $\longrightarrow$ & $17.31 \%$ & $0.81[0.38,1.24]$ \\
\hline Schinke 2009b & 415 & 413 & $-0.3(0.07)$ & $\rightarrow$ & $23.37 \%$ & $-0.3[-0.44,-0.16]$ \\
\hline Winters 2012 & 114 & 48 & $-0.2(0.172)$ & $\longrightarrow$ & $19.5 \%$ & $-0.2[-0.54,0.14]$ \\
\hline Total $(95 \% \mathrm{Cl})$ & & & & & $100 \%$ & $0.09[-0.24,0.43]$ \\
\hline \multicolumn{7}{|c|}{ Heterogeneity: $\operatorname{Tau}^{2}=0.12 ; \mathrm{Chi}^{2}=30.54, \mathrm{df}=4(\mathrm{P}<0.0001) ; \mathrm{I}^{2}=86.9 \%$} \\
\hline \multicolumn{7}{|c|}{ Test for overall effect: $Z=0.54(P=0.59)$} \\
\hline
\end{tabular}

Analysis 1.13. Comparison 1 Any parent/family intervention vs no intervention/ standard care, Outcome 13 Subgroup_universal_Alcohol use_Frequency.

\begin{tabular}{|c|c|c|c|c|c|c|c|}
\hline \multirow{3}{*}{$\begin{array}{l}\text { Study or subgroup } \\
\text { Linakis } 2013\end{array}$} & \multirow{3}{*}{$\begin{array}{c}\begin{array}{c}\text { Univer- } \\
\text { sal inter- } \\
\text { vention } \\
\text { N }\end{array} \\
44\end{array}$} & \multirow{3}{*}{$\begin{array}{l}\begin{array}{l}\text { No inter- } \\
\text { vention }\end{array} \\
\qquad \begin{array}{r}\text { N } \\
45\end{array}\end{array}$} & \multirow{3}{*}{$\begin{array}{c}\begin{array}{c}\text { Std. Mean } \\
\text { Difference }\end{array} \\
\text { (SE) } \\
0.8(0.221)\end{array}$} & \multirow{2}{*}{\multicolumn{2}{|c|}{$\begin{array}{l}\text { Std. Mean Difference } \\
\text { IV, Random, } 95 \% \mathrm{CI}\end{array}$}} & \multirow{3}{*}{$\begin{array}{l}\text { Weight } \\
30.47 \%\end{array}$} & \multirow{3}{*}{$\begin{array}{l}\text { Std. Mean Difference } \\
\text { IV, Random, 95\% Cl } \\
0.81[0.38,1.24]\end{array}$} \\
\hline & & & & & & & \\
\hline & & & & \multirow{3}{*}{ \# } & \multirow[b]{3}{*}{$\#$} & & \\
\hline Schinke 2009b & 415 & 413 & $-0.3(0.07)$ & & & $36.04 \%$ & $-0.3[-0.44,-0.16]$ \\
\hline Wurdak 2017 & 84 & 89 & $0.1(0.152)$ & & & $33.49 \%$ & $0.11[-0.19,0.41]$ \\
\hline
\end{tabular}




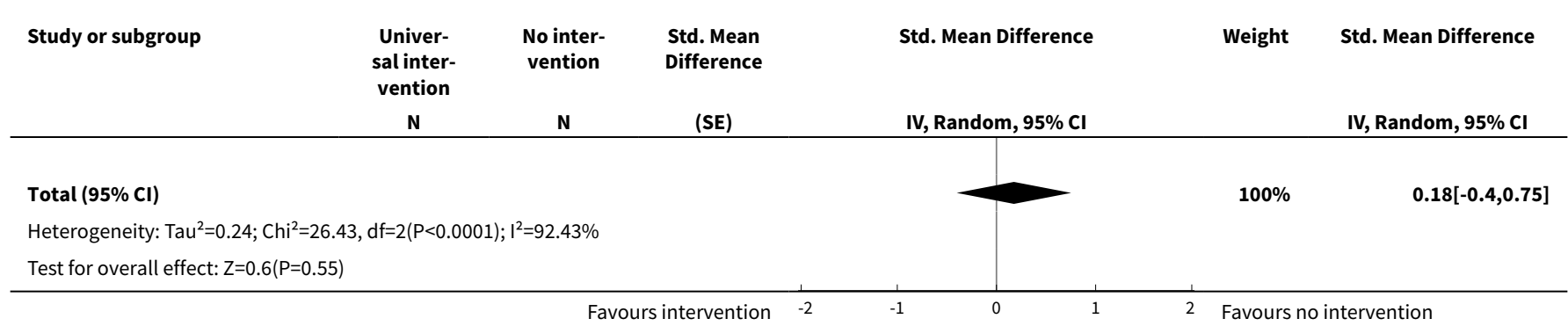

Analysis 1.14. Comparison 1 Any parent/family intervention vs no intervention/ standard care, Outcome 14 Subgroup_selective/indicated_Alcohol use_Frequency.

\begin{tabular}{|c|c|c|c|c|c|c|c|}
\hline Study or subgroup & $\begin{array}{l}\text { S/I Inter- } \\
\text { vention } \\
\mathbf{N}\end{array}$ & $\begin{array}{c}\text { No inter- } \\
\text { vention } \\
\mathbf{N}\end{array}$ & $\begin{array}{c}\text { Std. Mean } \\
\text { Difference } \\
\quad(\mathrm{SE})\end{array}$ & & $\begin{array}{l}\text { Std. Mean Difference } \\
\text { IV, Random, } 95 \% \mathrm{CI}\end{array}$ & Weight & $\begin{array}{l}\text { Std. Mean Difference } \\
\text { IV, Random, } 95 \% \mathrm{CI}\end{array}$ \\
\hline Arnaud 2016 & 141 & 175 & $0(0.113)$ & & + & $20.59 \%$ & $0.04[-0.18,0.26]$ \\
\hline Fang 2010 & 50 & 43 & $0.3(0.209)$ & & 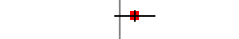 & $20.09 \%$ & $0.3[-0.11,0.71]$ \\
\hline Stanger 2017 & 28 & 30 & $0.1(0.263)$ & & 7 & $19.69 \%$ & $0.1[-0.41,0.62]$ \\
\hline Valdez 2013 & 54 & 62 & $-3.6(0.306)$ & $\multimap$ & & $19.33 \%$ & $-3.63[-4.23,-3.03]$ \\
\hline \multicolumn{6}{|l|}{ Total $(95 \% \mathrm{Cl})$} & $100 \%$ & $-0.65[-1.64,0.33]$ \\
\hline \multicolumn{8}{|c|}{ Heterogeneity: $\mathrm{Tau}^{2}=1.22 ; \mathrm{Chi}^{2}=137.29, \mathrm{df}=4(\mathrm{P}<0.0001) ; \mathrm{I}^{2}=97.09 \%$} \\
\hline \multicolumn{8}{|c|}{ Test for overall effect: $Z=1.3(P=0.19)$} \\
\hline
\end{tabular}

\section{Analysis 1.15. Comparison 1 Any parent/family intervention vs no intervention/ standard care, Outcome 15 Subgroup_>12 months_Alcohol use_Frequency.}

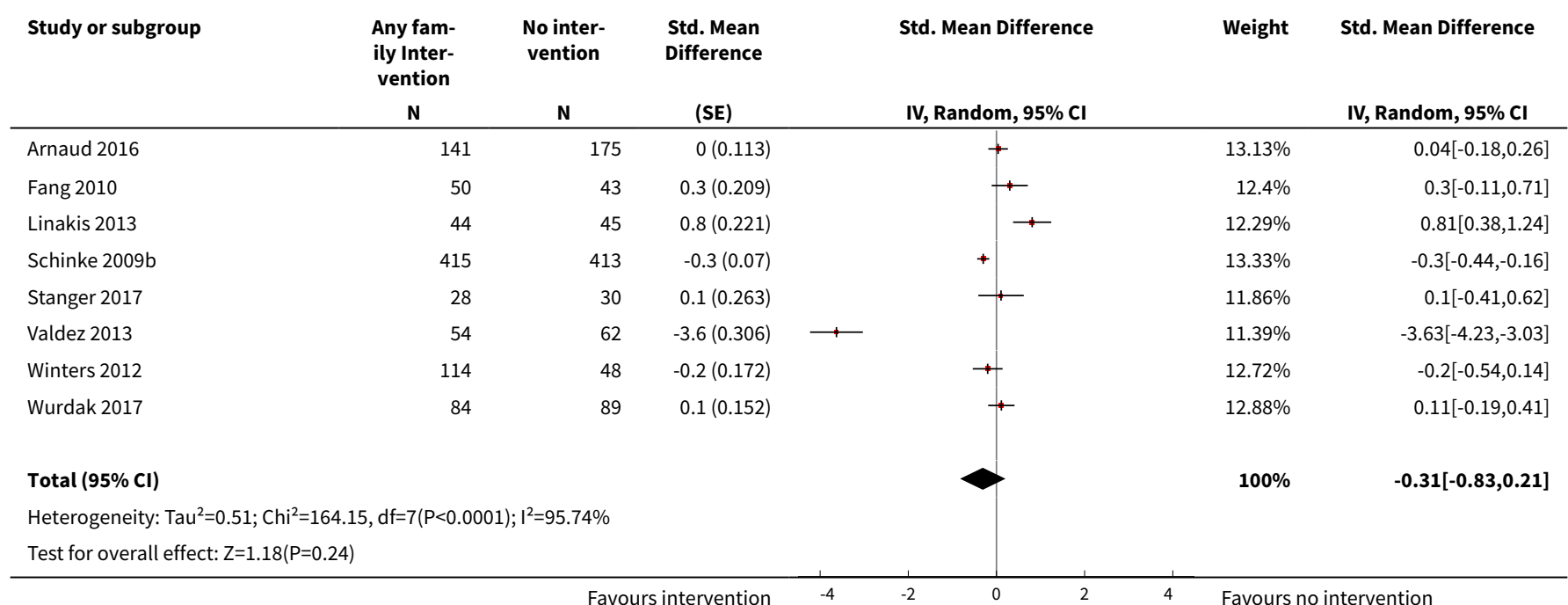


Analysis 1.16. Comparison 1 Any parent/family intervention vs no intervention/ standard care, Outcome 16 Subgroup_ethnicity minority_Alcohol use_Frequency.

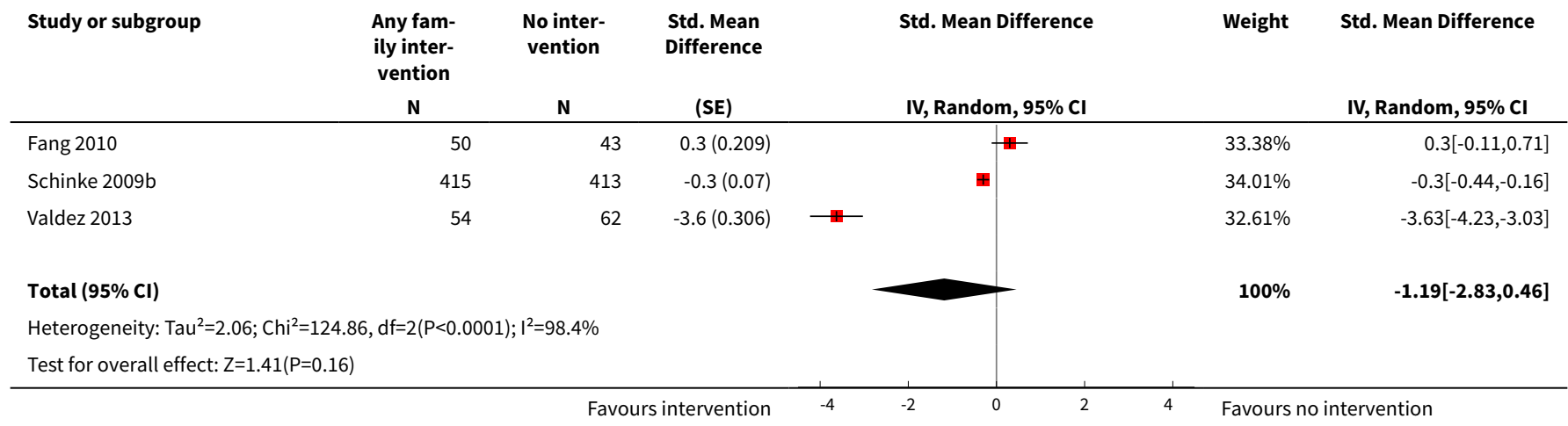

Analysis 1.17. Comparison 1 Any parent/family intervention vs no intervention/standard care, Outcome 17 Subgroup_ethnicity majority/caucasian_Alcohol use_Frequency.

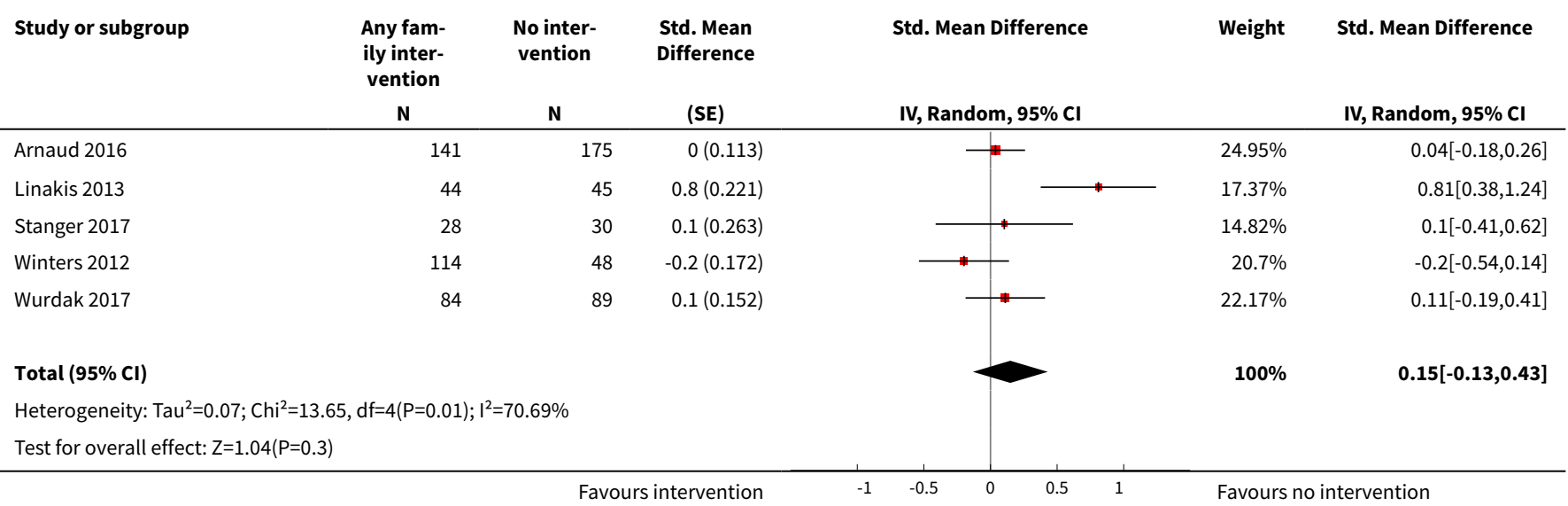

Analysis 1.18. Comparison 1 Any parent/family intervention vs no intervention/ standard care, Outcome 18 Subgroup_no binge_Alcohol use_Frequency.

\begin{tabular}{|c|c|c|c|c|c|c|c|}
\hline \multirow[t]{2}{*}{ Study or subgroup } & \multirow[t]{2}{*}{$\begin{array}{l}\text { Any fam- } \\
\text { ily inter- } \\
\text { vention } \\
\mathrm{N} \\
\end{array}$} & \multirow[t]{2}{*}{$\begin{array}{c}\text { No inter- } \\
\text { vention } \\
\text { N } \\
\end{array}$} & \multirow[t]{2}{*}{$\begin{array}{c}\text { Std. Mean } \\
\text { Difference } \\
\text { (SE) } \\
\end{array}$} & \multirow{2}{*}{\multicolumn{2}{|c|}{$\begin{array}{l}\text { Std. Mean Difference } \\
\text { IV, Random, 95\% CI }\end{array}$}} & Weight & \multirow[t]{2}{*}{$\begin{array}{l}\text { Std. Mean Difference } \\
\text { IV, Random, 95\% CI }\end{array}$} \\
\hline & & & & & & & \\
\hline Fang 2010 & 50 & 43 & $0.3(0.209)$ & & + & $14.28 \%$ & $0.3[-0.11,0.71]$ \\
\hline Linakis 2013 & 44 & 45 & $0.8(0.221)$ & & $\rightarrow$ & $14.19 \%$ & $0.81[0.38,1.24]$ \\
\hline Schinke 2009b & 415 & 413 & $-0.3(0.07)$ & & + & $15.07 \%$ & $-0.3[-0.44,-0.16]$ \\
\hline Stanger 2017 & 28 & 30 & $0.1(0.263)$ & & $\rightarrow$ & $13.82 \%$ & $0.1[-0.41,0.62]$ \\
\hline Valdez 2013 & 54 & 62 & $-3.6(0.306)$ & $\longrightarrow$ & & $13.4 \%$ & $-3.63[-4.23,-3.03]$ \\
\hline Winters 2012 & 114 & 48 & $-0.2(0.172)$ & & $\rightarrow$ & $14.56 \%$ & $-0.2[-0.54,0.14]$ \\
\hline Wurdak 2017 & 84 & 89 & $0.1(0.152)$ & & + & $14.69 \%$ & $0.11[-0.19,0.41]$ \\
\hline Total $(95 \% \mathrm{Cl})$ & & & & & & $100 \%$ & $-0.37[-1.01,0.27]$ \\
\hline \multicolumn{8}{|c|}{ Heterogeneity: $\mathrm{Tau}^{2}=0.71 ; \mathrm{Chi}^{2}=159.9, \mathrm{df}=6(\mathrm{P}<0.0001) ; \mathrm{I}^{2}=96.25 \%$} \\
\hline
\end{tabular}




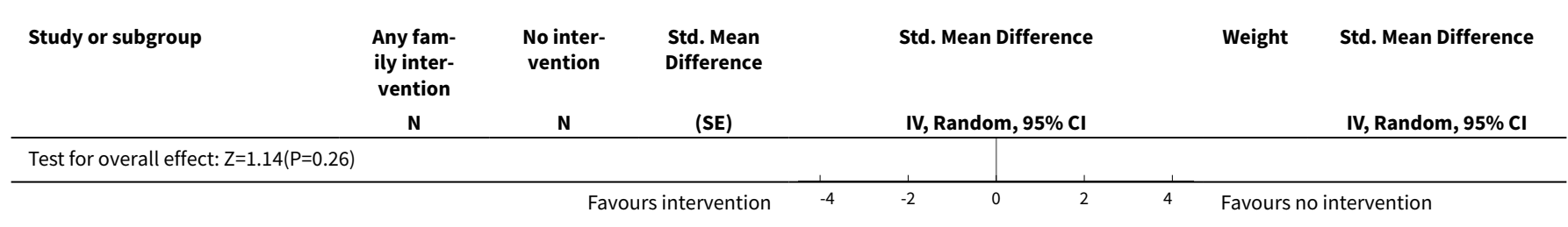

Analysis 1.19. Comparison 1 Any parent/family intervention vs no intervention/standard care, Outcome 19 Sensitivity_RoB_Alcohol use_Volume.

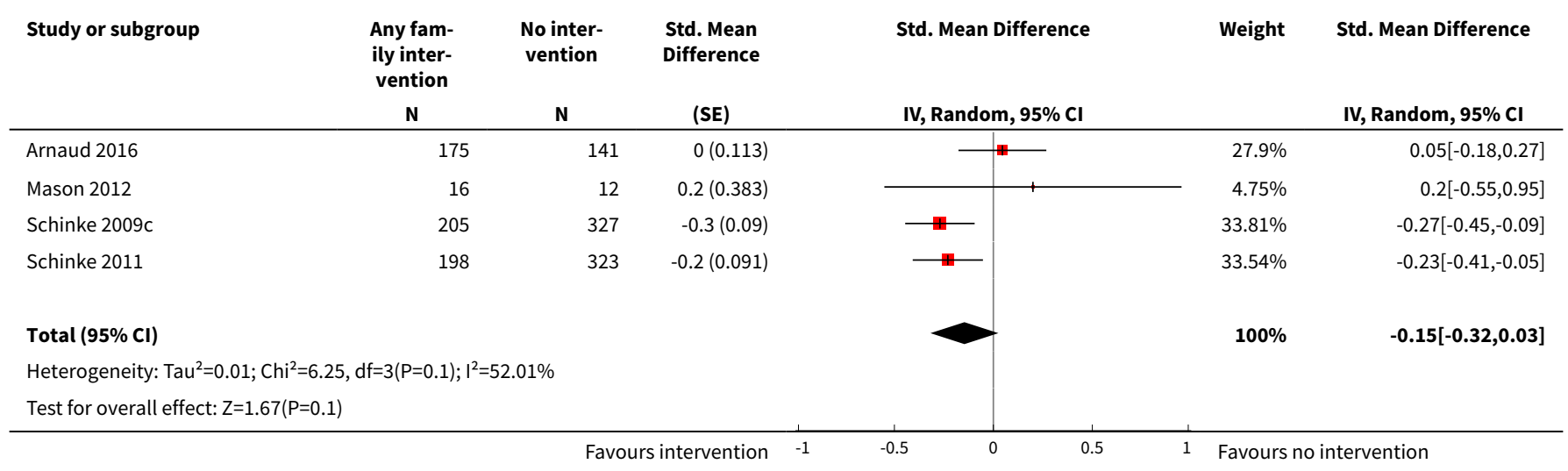

Analysis 1.20. Comparison 1 Any parent/family intervention vs no intervention/ standard care, Outcome 20 Subgroup_universal_Alcohol use_Volume.

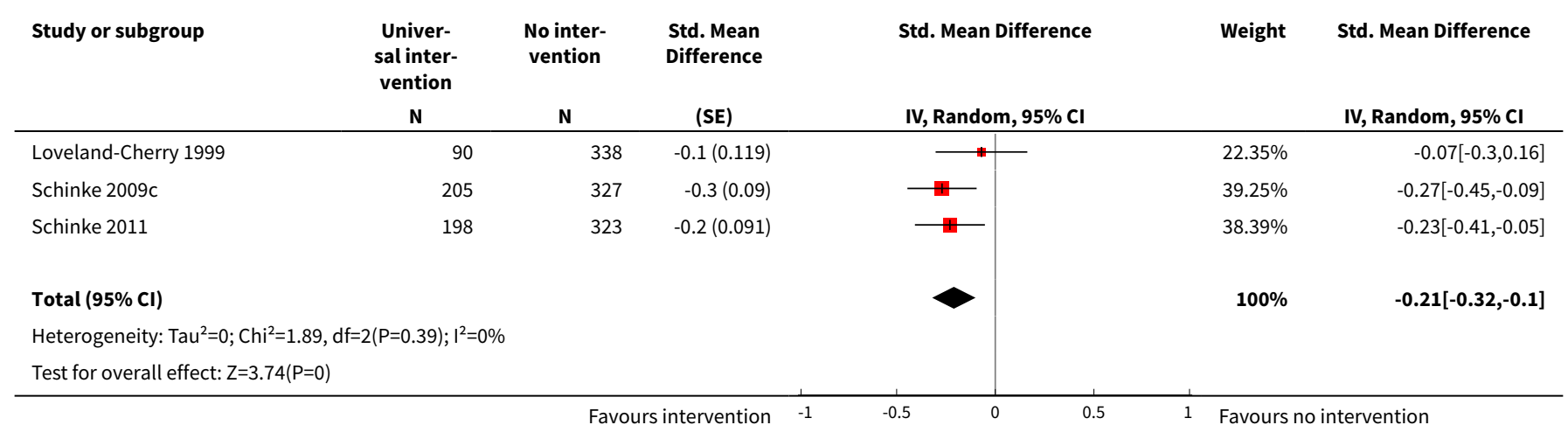

Analysis 1.21. Comparison 1 Any parent/family intervention vs no intervention/ standard care, Outcome 21 Subgroup_selective/indicated_Alcohol use_Volume.

\begin{tabular}{|c|c|c|c|c|c|c|}
\hline Study or subgroup & $\begin{array}{c}\text { S_I_inter- } \\
\text { vention } \\
\mathbf{N} \\
\end{array}$ & $\begin{array}{c}\text { No inter- } \\
\text { vention } \\
\mathbf{N}\end{array}$ & $\begin{array}{l}\text { Std. Mean } \\
\text { Difference } \\
\text { (SE) }\end{array}$ & $\begin{array}{l}\text { Std. Mean Difference } \\
\text { IV, Random, 95\% CI }\end{array}$ & Weight & $\begin{array}{l}\text { Std. Mean Difference } \\
\text { IV, Random, } 95 \% \mathrm{CI}\end{array}$ \\
\hline Arnaud 2016 & 175 & 141 & $0(0.113)$ & 1 & $91.97 \%$ & $0.05[-0.18,0.27]$ \\
\hline Mason 2012 & 16 & 12 & $0.2(0.383)$ & & $8.03 \%$ & $0.2[-0.55,0.95]$ \\
\hline
\end{tabular}




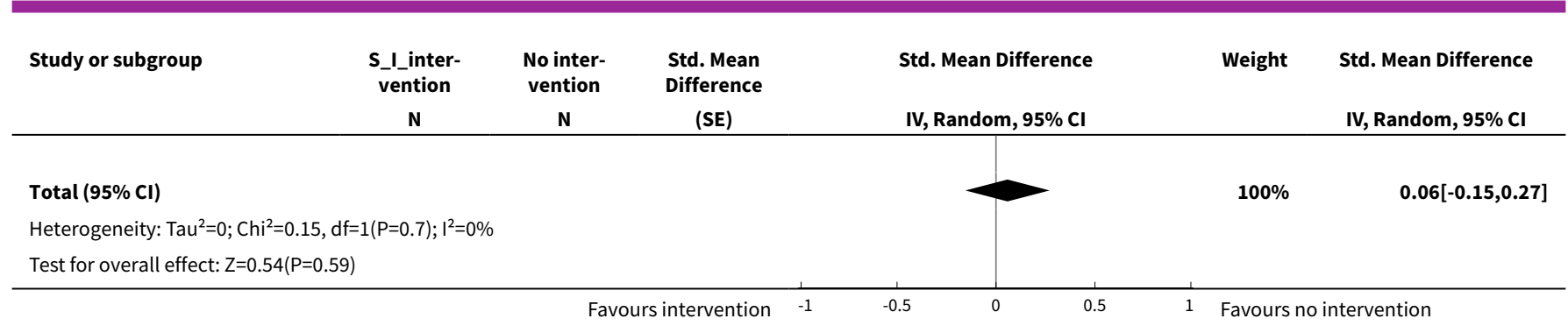

\section{Analysis 1.22. Comparison 1 Any parent/family intervention vs no intervention/ standard care, Outcome 22 Subgroup_>12 months_Alcohol use_Volume.}

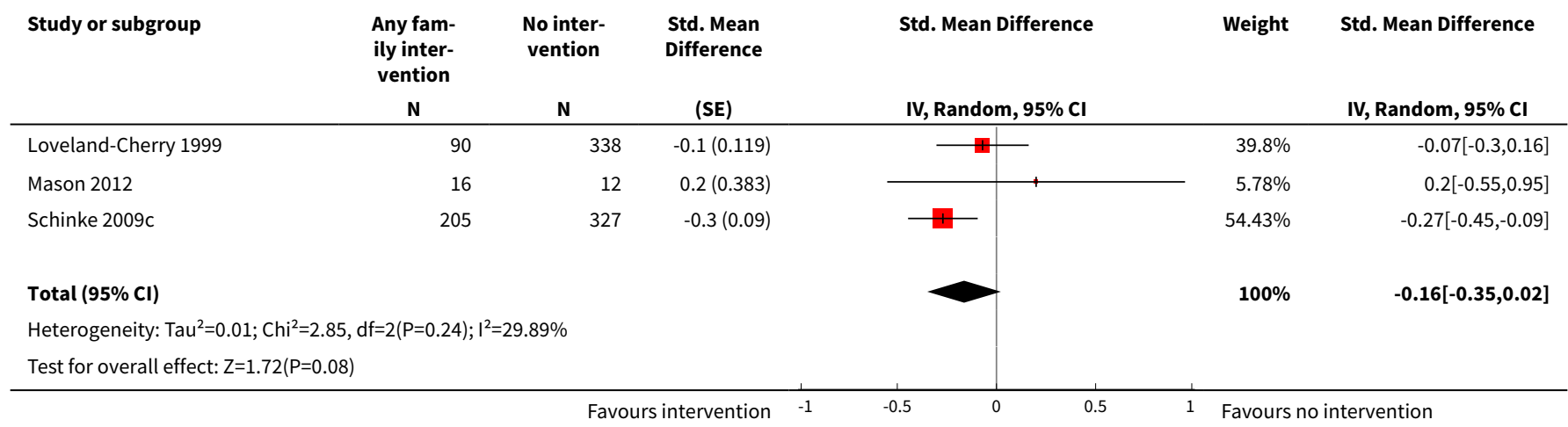

Analysis 1.23. Comparison 1 Any parent/family intervention vs no intervention/ standard care, Outcome 23 Subgroup_ethnicity_minority_Alcohol use_Volume.

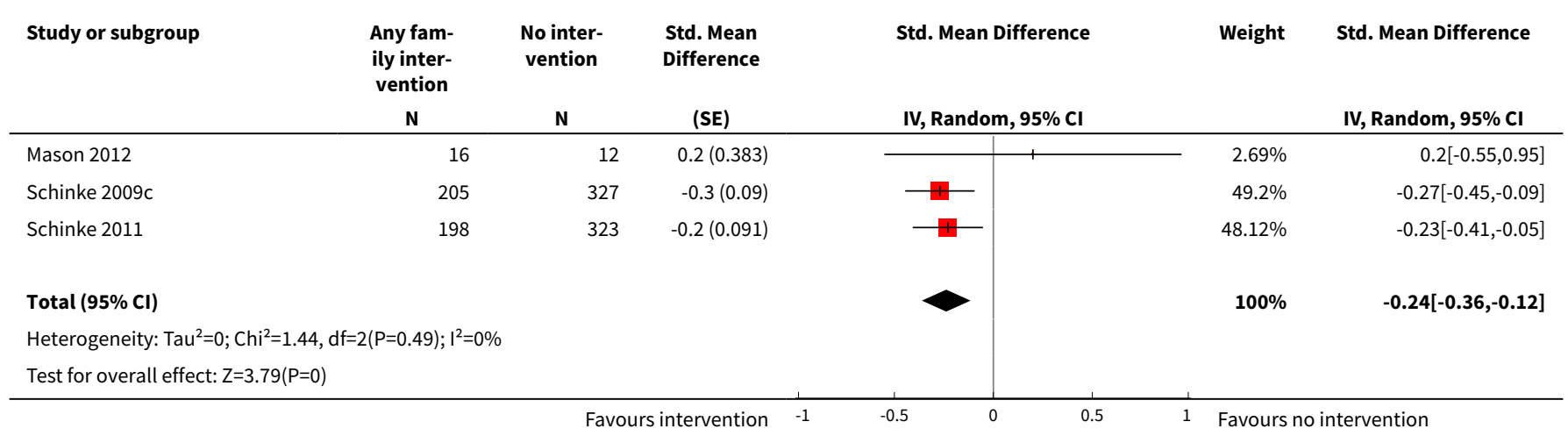


Analysis 1.24. Comparison 1 Any parent/family intervention vs no intervention/ standard care, Outcome 24 Subgroup_ethnicity majority/caucasian_Alcohol use_Volume.

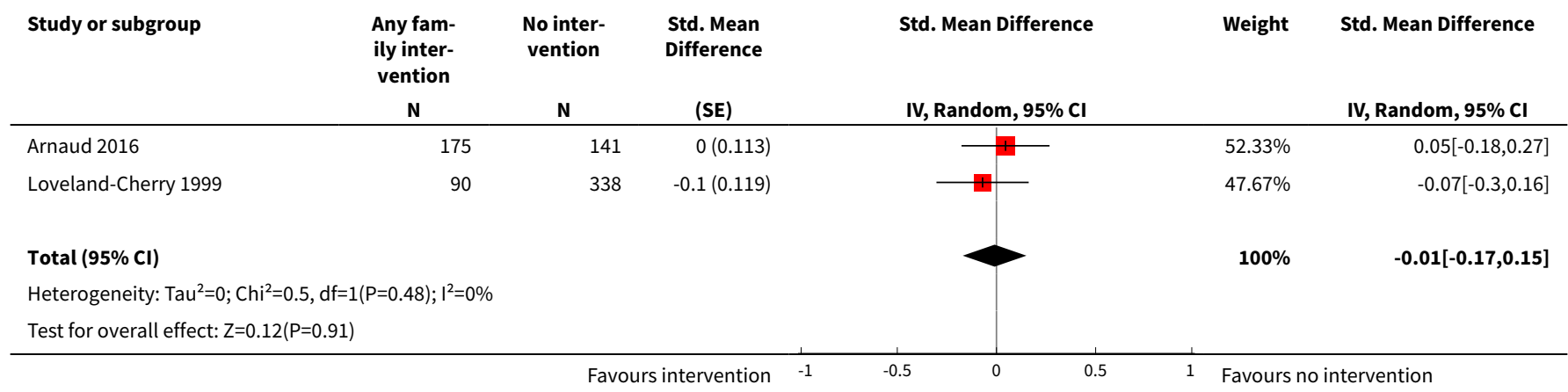

Analysis 1.25. Comparison 1 Any parent/family intervention vs no intervention/ standard care, Outcome 25 Subgroup_female only_Alcohol use_Volume.

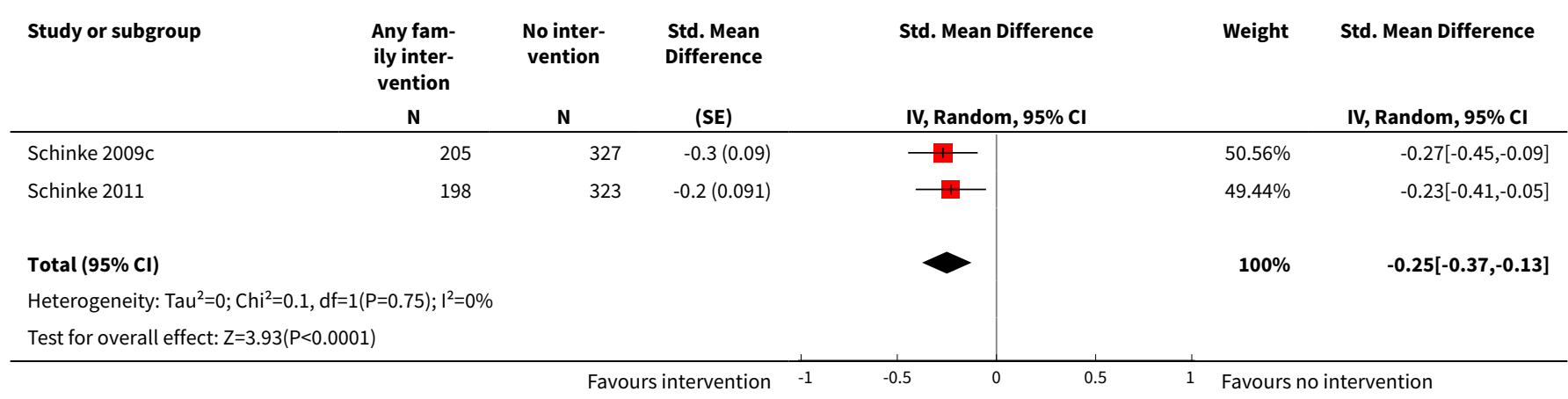

Comparison 2. Family-based and adolescent intervention vs intervention with young people alone

\begin{tabular}{lllll}
\hline Outcome or subgroup title & $\begin{array}{l}\text { No. of } \\
\text { studies }\end{array}$ & $\begin{array}{l}\text { No. of } \\
\text { partici- } \\
\text { pants }\end{array}$ & Statistical method & Effect size \\
\hline 1 Alcohol use_prevalence & 4 & 5640 & Std. Mean Difference (Random, 95\% Cl) & $-0.39[-0.91,0.14]$ \\
\hline 2 Alcohol use_frequency & 4 & 915 & Std. Mean Difference (Random, 95\% Cl) & $-0.16[-0.42,0.09]$ \\
\hline $\begin{array}{l}\text { 3 Sensitivity_RoB_Alcohol } \\
\text { use_prevalence }\end{array}$ & 2 & 3891 & Std. Mean Difference (Random, 95\% Cl) & $-0.61[-1.84,0.63]$ \\
\hline $\begin{array}{l}\text { 4 Subgroup_universal_Alcohol } \\
\text { use_prevalence }\end{array}$ & 3 & 5351 & Std. Mean Difference (Random, 95\% Cl) & $-0.44[-1.08,0.20]$ \\
\hline $\begin{array}{l}\text { 5 Sensitivity_RoB_Alcohol } \\
\text { use_frequency }\end{array}$ & 3 & 832 & Std. Mean Difference (Random, 95\% Cl) & $-0.21[-0.50,0.08]$ \\
\hline $\begin{array}{l}\text { 6 Subgroup_universal_Alcohol } \\
\text { use_frequency }\end{array}$ & 2 & 596 & Std. Mean Difference (Random, 95\% Cl) & $-0.30[-0.68,0.07]$ \\
\hline
\end{tabular}




\begin{tabular}{lllll}
\hline Outcome or subgroup title & $\begin{array}{l}\text { No. of } \\
\text { studies }\end{array}$ & $\begin{array}{l}\text { No. of } \\
\text { partici- } \\
\text { pants }\end{array}$ & Statistical method & Effect size \\
\hline $\begin{array}{l}7 \text { Subgroup_indicated_Alcohol } \\
\text { use_frequency }\end{array}$ & 2 & 319 & Std. Mean Difference (Random, 95\% Cl) & $0.01[-0.21,0.23]$ \\
\hline
\end{tabular}

Analysis 2.1. Comparison 2 Family-based and adolescent intervention vs intervention with young people alone, Outcome 1 Alcohol use_prevalence.

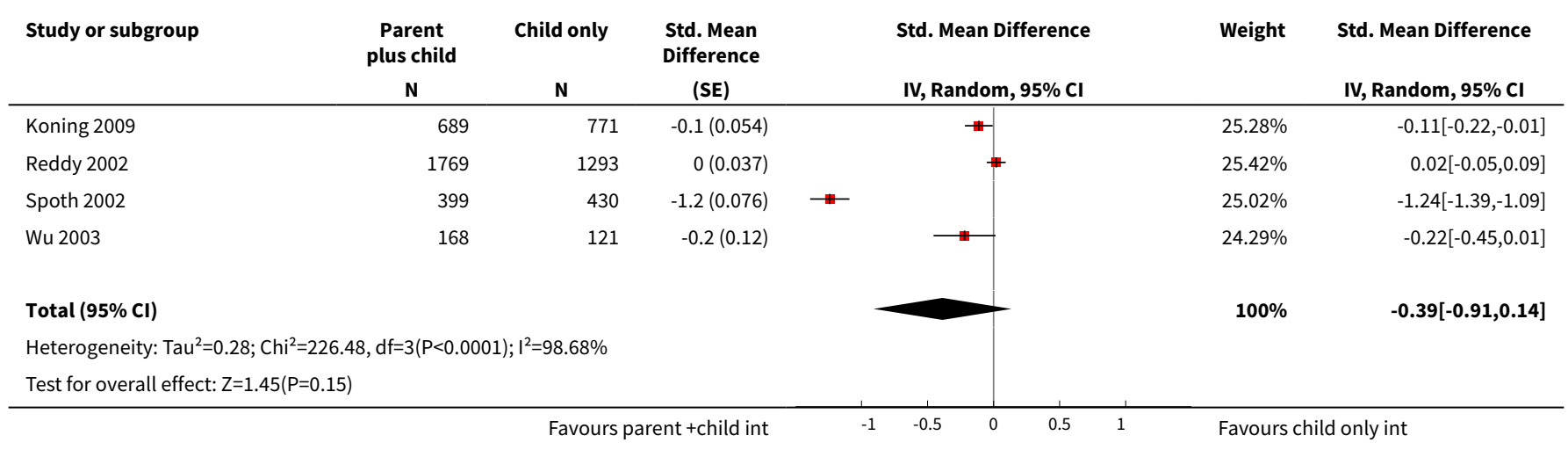

Analysis 2.2. Comparison 2 Family-based and adolescent intervention vs intervention with young people alone, Outcome 2 Alcohol use_frequency.

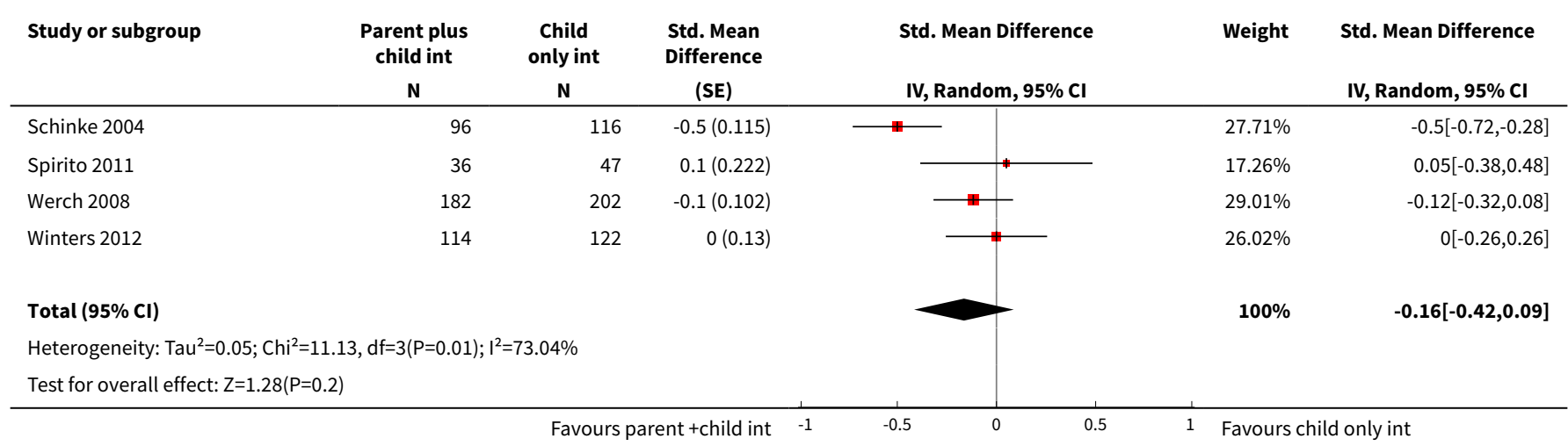

Analysis 2.3. Comparison 2 Family-based and adolescent intervention vs intervention with young people alone, Outcome 3 Sensitivity_RoB_Alcohol use_prevalence.

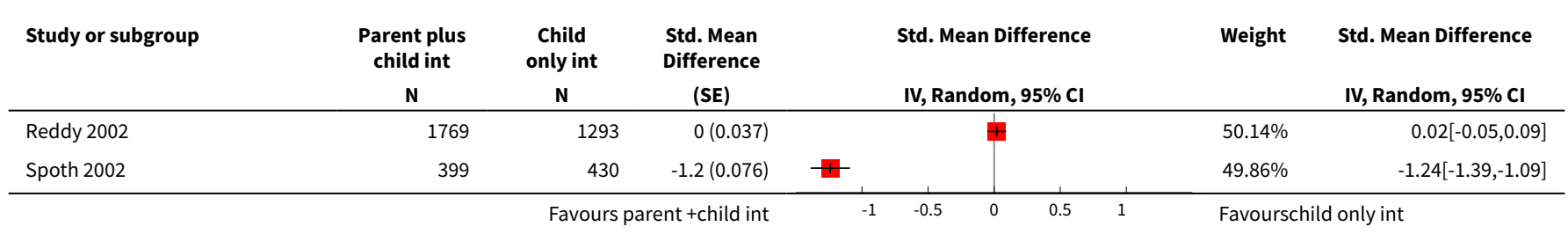




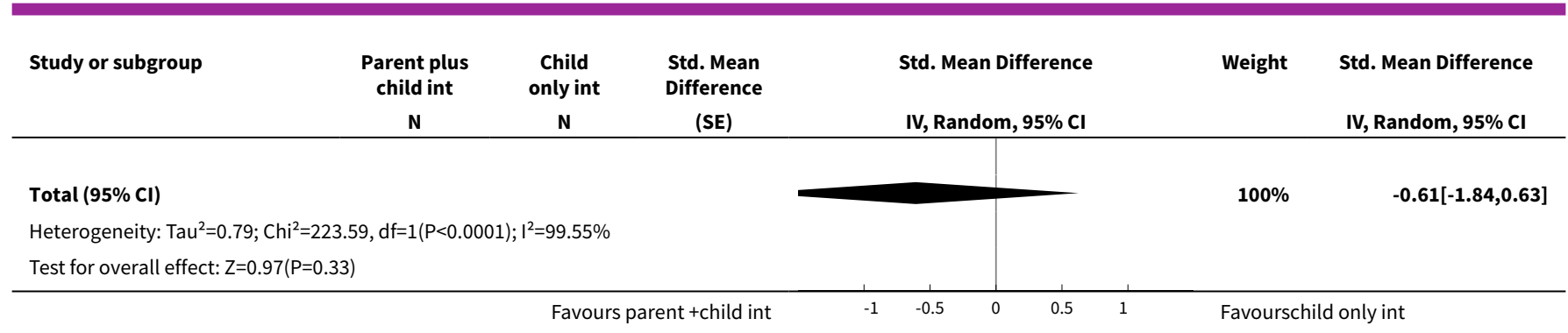

Analysis 2.4. Comparison 2 Family-based and adolescent intervention vs intervention with young people alone, Outcome 4 Subgroup_universal_Alcohol use_prevalence.

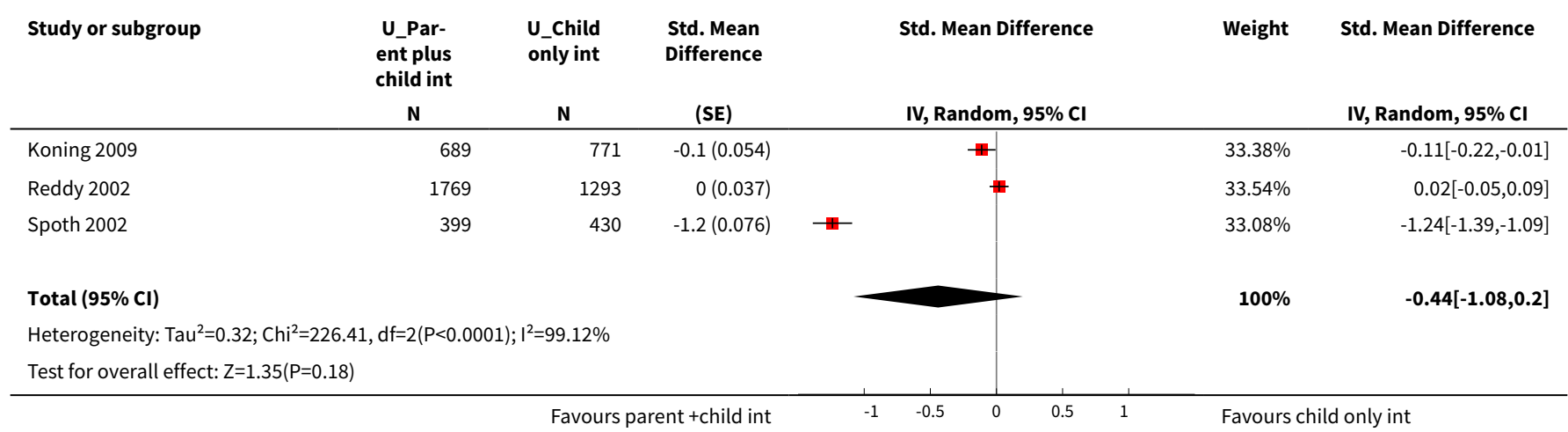

Analysis 2.5. Comparison 2 Family-based and adolescent intervention vs intervention with young people alone, Outcome 5 Sensitivity_RoB_Alcohol use_frequency.

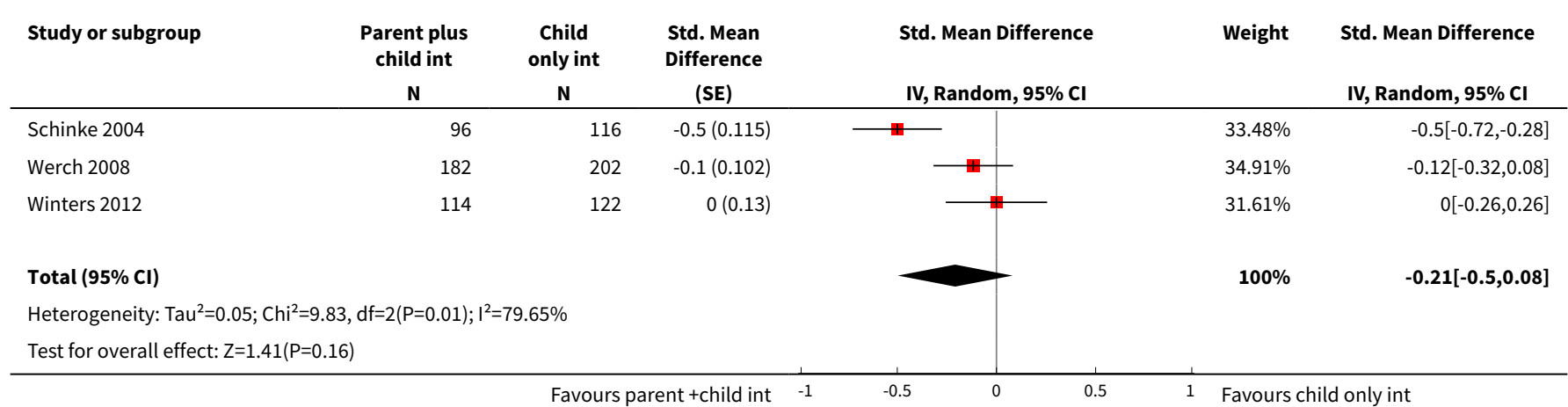

Analysis 2.6. Comparison 2 Family-based and adolescent intervention vs intervention with young people alone, Outcome 6 Subgroup_universal_Alcohol use_frequency.

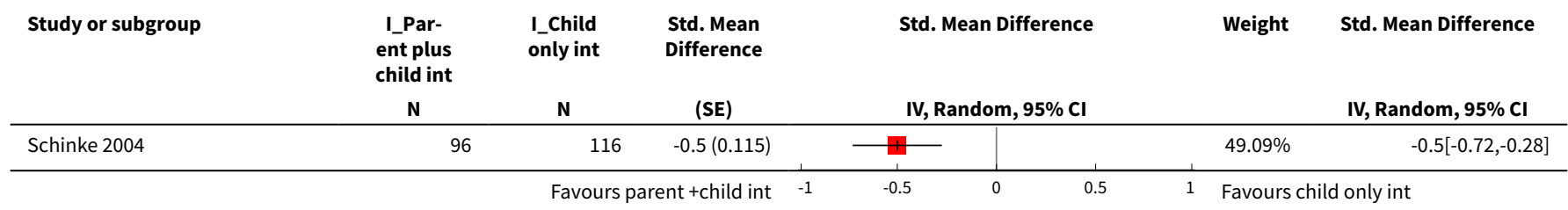




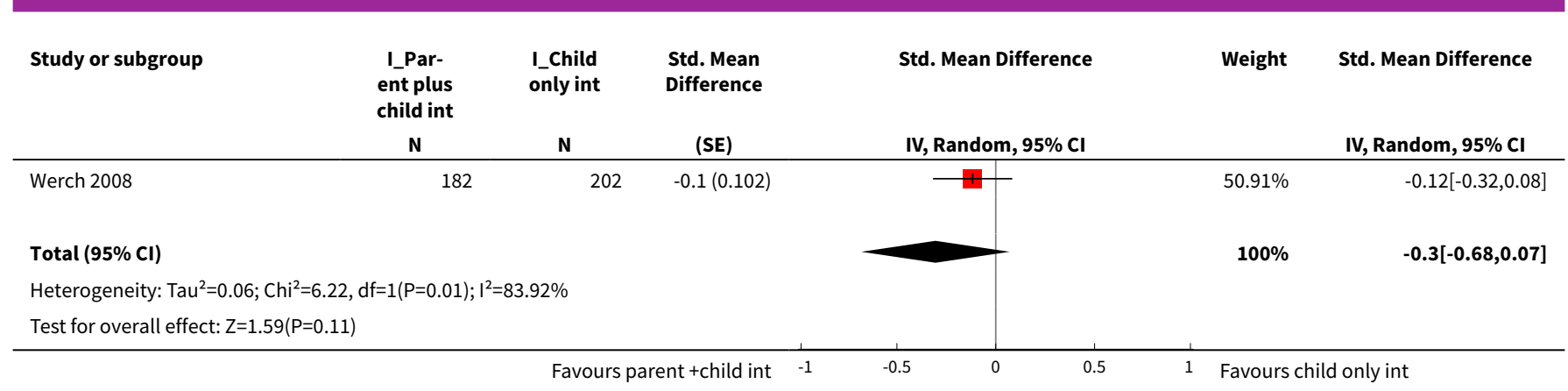

\section{Analysis 2.7. Comparison 2 Family-based and adolescent intervention vs intervention with young people alone, Outcome 7 Subgroup_indicated_Alcohol use_frequency.}

\begin{tabular}{|c|c|c|c|c|c|c|}
\hline Study or subgroup & $\begin{array}{c}\text { I_Par- } \\
\text { ent plus } \\
\text { child int } \\
N\end{array}$ & $\begin{array}{c}\text { I_Child } \\
\text { only int } \\
\text { N }\end{array}$ & $\begin{array}{c}\text { Std. Mean } \\
\text { Difference } \\
\text { (SE) }\end{array}$ & IV, Random, 95\% CI & Weight & IV, Random, 95\% CI \\
\hline Spirito 2011 & 36 & 47 & $0.1(0.222)$ & - & $25.71 \%$ & $0.05[-0.38,0.48]$ \\
\hline Winters 2012 & 114 & 122 & $0(0.13)$ & & $74.29 \%$ & $0[-0.26,0.26]$ \\
\hline Total $(95 \% \mathrm{Cl})$ & & & & & $100 \%$ & $0.01[-0.21,0.23]$ \\
\hline \multicolumn{7}{|c|}{ Heterogeneity: $\mathrm{Tau}^{2}=0 ; \mathrm{Chi}^{2}=0.04, \mathrm{df}=1(\mathrm{P}=0.85) ; \mathrm{I}^{2}=0 \%$} \\
\hline \multicolumn{7}{|c|}{ Test for overall effect: $\mathrm{Z}=0.11(\mathrm{P}=0.91)$} \\
\hline
\end{tabular}

\section{APPENDICES}

\section{Appendix 1. Search strategies}

\section{Explanation of symbols:}

.tw OVID Databases - searches across the title and abstract fields

adj OVID Databases - adjacency searching. For example, "adj5" will retrieve the nominated words within 5 words of each other, in any order near CENTRAL, and ERIC - adjacency searching. For example, "near/5" will retrieve the nominated words within 5 words of each other, in any order

/ OVID Databases - a slash appearing after a search word/phrase indicates search within the subject heading field

* All Databases - truncation symbol - will retrieve all words beginning with the set of letters appearing before the symbol

exp OVID Databases - indicates the search has been exploded to include all sub-topics

tw OVID databases - searches within the title and abstract fields

ti, ab CENTRAL - searches within the title and abstract fields

mp OVID Databases - searches across all text fields

\section{MEDLINE search strategy:}

1 Randomized Controlled Trial/

2 Controlled Clinical Trial/ 
3 Random Allocation/

4 Double-Blind Method/

5 Single-Blind Method/

6 clinical trial/

7 ((singl ${ }^{\star}$ or doubl ${ }^{\star}$ or trebl ${ }^{\star}$ or tripl $\left.{ }^{\star}\right)$ adj5 (blind ${ }^{\star}$ or mask $\left.\left.^{\star}\right)\right)$.tw.

8 Placebos/

9 placebo*.tw.

10 trial.tw.

11 random*.tw.

12 groups.tw.

13 Research Design/

141 or 2 or 3 or 4 or 5 or 6 or 7 or 8 or 9 or 10 or 11 or 12 or 13

15 exp Alcohols/ad, ae, to [Administration \& Dosage, Adverse Effects, Toxicity]

16 exp Alcohol Drinking/

17 Alcoholism/

18 Alcoholic Intoxication/

19 exp Alcoholic Beverages/

20 alcohol $^{*}$.tw.

21 drink $^{*}$. tw.

22 drunk $^{*}$. tw.

23 intoxicat ${ }^{\star}$. tw.

24 binge ${ }^{\star}$. tw.

25 Alcoholics/

2615 or 16 or 17 or 18 or 19 or 20 or 21 or 22 or 23 or 24 or 25

27 adolescent/ or exp child/ or exp infant/

28 Students/

29 (youth* or juvenile* or adolescen $^{\star}$ or teen ${ }^{\star}$ or schoolchild* or girl ${ }^{\star}$ or boy ${ }^{\star}$ or minor ${ }^{\star}$ ).tw.

30 (young* adj2 (adult* or people or person $\left.\left.{ }^{\star}\right)\right) . t w$.

31 (early adj2 adult $\left.^{\star}\right)$.tw.

3227 or 28 or 29 or 30 or 31

33 intervention ${ }^{\star}$. tw.

34 educat $^{\star}$. tw.

35 promot $^{\star}$. tw.

36 adverti ${ }^{\star}$. tw.

37 campaign $^{\star}$. tw.

Family-based prevention programmes for alcohol use in young people (Review) 
38 (mass adj2 media).tw.

39 (prevention adj5 (primary or secondary or universal or selective or target* or indicated)).tw.

40 Health Education/ or Patient Education as Topic/ or Education/

4133 or 34 or 35 or 36 or 37 or 38 or 39 or 40

4214 and 26 and 32 and 41

43 (animals not (humans and animals)).sh.

4442 not 43

\section{Embase Classic + Embase search strategy:}

1 randomized controlled trial/

2 controlled clinical trial/

3 randomization/

4 double blind procedure/

5 single blind procedure/

6 clinical trial/

7 ((singl ${ }^{\star}$ or doubl ${ }^{\star}$ or trebl* or tripl $\left.{ }^{\star}\right) \operatorname{adj5}\left(\right.$ blind $^{\star}$ or mask $\left.\left.^{\star}\right)\right)$. tw.

8 placebo/

9 placebo*.tw.

10 trial.tw.

11 random*.tw.

12 groups.tw.

13 Research Design.mp. or methodology/

141 or 2 or 3 or 4 or 5 or 6 or 7 or 8 or 9 or 10 or 11 or 12 or 13

15 exp alcohol derivative/ae, ad, do, to [Adverse Drug Reaction, Drug Administration, Drug Dose, Drug Toxicity]

16 alcohol/ae, ad, do, to [Adverse Drug Reaction, Drug Administration, Drug Dose, Drug Toxicity]

17 drinking behavior/

18 alcoholism/

19 alcohol intoxication/

20 exp alcoholic beverage/

21 alcohol $^{*}$. tw.

22 drink $^{*}$. tw.

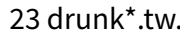

24 intoxicat*.tw.

25 binge ${ }^{\star} . t w$.

2615 or 16 or 17 or 18 or 19 or 20 or 21 or 22 or 23 or 24 or 25

27 adolescent/ or exp child/ or exp infant/

Family-based prevention programmes for alcohol use in young people (Review) 
28 student/

29 (youth $^{\star}$ or juvenile ${ }^{\star}$ or adolescen ${ }^{\star}$ or teen ${ }^{\star}$ or schoolchild ${ }^{\star}$ or girl ${ }^{\star}$ or boy ${ }^{\star}$ or minor ${ }^{\star}$ ).tw.

30 (young* adj2 (adult* or people or person $\left.{ }^{\star}\right)$ ).tw.

31 (early adj2 adult $\left.^{\star}\right)$.tw.

3227 or 28 or 29 or 30 or 31

33 intervention ${ }^{\star}$. tw.

34 educat $^{\star}$.tw.

35 promot $^{\star}$. tw .

36 adverti*.tw.

37 campaign $^{\star}$. tw.

38 (mass adj2 media).tw.

39 (prevention adj5 (primary or secondary or universal or selective or target* or indicated)).tw.

40 patient education/ or education/ or health education/

4133 or 34 or 35 or 36 or 37 or 38 or 39 or 40

4214 and 26 and 32 and 41

43 (animal not (humans and animal)).sh.

4442 not 43

\section{PsycINFO search strategy:}

1 Clinical Trials/

2 Random Sampling/

$3\left(\left(\right.\right.$ singl ${ }^{\star}$ or doubl $^{\star}$ or trebl ${ }^{\star}$ or tripl $\left.l^{\star}\right) \operatorname{adj5}\left(\right.$ blind $^{\star}$ or mask $\left.\left.^{\star}\right)\right)$. tw.

4 placebo/

5 placebo*.tw.

6 trial.tw.

7 random ${ }^{\star}$. tw.

8 groups.tw.

9 Research Design.mp. or exp Experimental Design/

101 or 2 or 3 or 4 or 5 or 6 or 7 or 8 or 9

$11 \exp$ ALCOHOLS/

12 exp Alcohol Drinking Patterns/

13 ALCOHOLISM/

14 exp Alcohol Intoxication/

15 exp Alcoholic Beverages/

16 alcohol* $^{*}$.tw.

17 drink $^{\star}$. tw.

Family-based prevention programmes for alcohol use in young people (Review) 
18 drunk $^{*}$. tw

19 intoxicat ${ }^{\star}$. tw.

20 binge ${ }^{\star}$. tw.

2111 or 12 or 13 or 14 or 15 or 16 or 17 or 18 or 19 or 20

22 exp STUDENTS/

23 (youth ${ }^{\star}$ or juvenile ${ }^{\star}$ or adolescen ${ }^{\star}$ or teen ${ }^{\star}$ or schoolchild ${ }^{\star}$ or girl ${ }^{\star}$ or boy ${ }^{\star}$ or minor ${ }^{\star}$ ).tw.

24 (young* adj2 (adult* or people or person $\left.\left.{ }^{\star}\right)\right) . t w$.

25 (early adj2 adult*).tw.

2622 or 23 or 24 or 25

27 intervention ${ }^{\star}$. tw.

28 educat*.tw.

29 promot $^{\star}$. tw.

30 adverti $^{*}$. tw.

31 campaign ${ }^{\star}$. tw.

32 (mass adj2 media).tw.

33 (prevention adj5 (primary or secondary or universal or selective or target* or indicated)).tw.

34 exp EDUCATION/ or exp HEALTH EDUCATION/

3527 or 28 or 29 or 30 or 31 or 32 or 33 or 34

3610 and 21 and 26 and 35

37 limit 36 to human

\section{MEDLINE In-Process \& Other Non-Indexed Citations search strategy:}

1 Randomized Controlled Trial/

2 Controlled Clinical Trial/

3 Clinical Trial/

4 ((singl ${ }^{\star}$ or doubl* or trebl* or tripl* $\left.{ }^{\star}\right) \operatorname{adj} 5\left(\right.$ blind $^{\star}$ or mask $\left.\left.{ }^{\star}\right)\right)$. tw.

5 placebo*.mp.

6 trial.mp.

7 random ${ }^{\star} . \mathrm{mp}$.

8 groups.mp.

9 Research Design.mp.

101 or 2 or 3 or 4 or 5 or 6 or 7 or 8 or 9

11 alcohol $^{\star} \cdot \mathrm{mp}$.

12 drink* $^{\star} . \mathrm{mp}$.

13 drunk $^{*} . \mathrm{mp}$.

14 intoxicat ${ }^{\star} . \mathrm{mp}$.

Family-based prevention programmes for alcohol use in young people (Review) 
15 binge ${ }^{\star} . \mathrm{mp}$.

\section{1 or 12 or 13 or 14 or 15}

17 Student $^{\star} . \mathrm{mp}$.

18 (youth* ${ }^{\star}$ or juvenile ${ }^{\star}$ or adolescen ${ }^{\star}$ or teen ${ }^{\star}$ or schoolchild ${ }^{\star}$ or girl ${ }^{\star}$ or boy ${ }^{\star}$ or minor ${ }^{\star}$ ).mp.

19 (young* adj2 (adult* or people or person $\left.\left.{ }^{\star}\right)\right) . m p$.

20 (early adj2 adult*).mp.

2117 or 18 or 19 or 20

22 intervention*.mp.

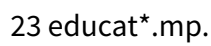

24 promot*$^{\star} \cdot \mathrm{mp}$.

25 adverti $^{\star} \cdot \mathrm{mp}$.

26 campaign ${ }^{\star} . \mathrm{mp}$.

27 (mass adj2 media).mp.

28 (prevention adj5 (primary or secondary or universal or selective or target* or indicated)).mp.

2922 or 23 or 24 or 25 or 26 or 27 or 28

3010 and 16 and 21 and 29 (human limit not applied to Medline in Process records)

\section{CENTRAL search strategy:}

\#1MeSH descriptor: [Alcohols] explode all trees

\#2 MeSH descriptor: [Alcohol Drinking] explode all trees

\#3 MeSH descriptor: [Alcoholism] this term only

\#4 MeSH descriptor: [Alcoholic Intoxication] this term only

\#5 MeSH descriptor: [Alcoholic Beverages] explode all trees

\#6 alcohol*:ti,ab

\#7 drink ${ }^{\star}:$ ti,ab

\#8 drunk*:ti,ab

\#9 intoxicat*:ti,ab

\#10 binge* $:$ ti,ab

\#11 MeSH descriptor: [Alcoholics] this term only

\#12 \{or\#1-\#11\}

\#13 MeSH descriptor: [Child] explode all trees

\#14 MeSH descriptor: [Infant] explode all trees

\#15 MeSH descriptor: [Adolescent] this term only

\#16 MeSH descriptor: [Students] this term only

\#17 (youth* or juvenile* or adolescen* or teen ${ }^{\star}$ or schoolchild* or girl ${ }^{\star}$ or boy ${ }^{\star}$ or minor $^{\star}$ ):ti,ab

\#18 (young* near/2 (adult* or people or person $\left.{ }^{\star}\right)$ ):ti,ab

Family-based prevention programmes for alcohol use in young people (Review) 
\#19 (early near/2 adult*):ti,ab

\#20 \{or \#13-\#19\}

\#21 intervention*:ti,ab

\#22 educat*:ti,ab

\#23 promot*:ti,ab

\#24 adverti*:ti,ab

\#25 campaign*:ti,ab

\#26 (mass near/2 media):ti,ab

\#27 (prevention near/5 (primary or secondary or universal or selective or target or indicated)):ti,ab

\#28 MeSH descriptor: [Education] explode all trees

\#29 MeSH descriptor: [Health Education] this term only

\#30 MeSH descriptor: [Patient Education as Topic] this term only

\#31 \{or \#21-\#30\}

$\# 32$ and \#12, \#20, \#31\}

ERIC search strategy:

\section{Separate searches in title, abstract, and subject headings fields}

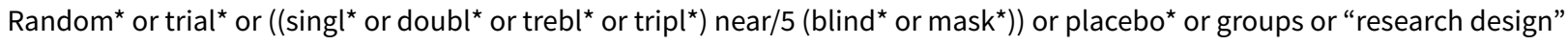

Alcohol $^{*}$ or drink ${ }^{\star}$ or drunk* or intoxicat* or binge*

youth $^{\star}$ or juvenile* or adolescen* or teen ${ }^{\star}$ or schoolchild ${ }^{\star}$ or girl ${ }^{\star}$ or boy ${ }^{\star}$ or minor ${ }^{\star}$ or (young near/2 (adult* or people or person $\left.^{\star}\right)$ ) or (early near/2 adult ${ }^{\star}$ )

intervention ${ }^{\star}$ or educat ${ }^{\star}$ or promot ${ }^{\star}$ or adverti ${ }^{\star}$ or campaign ${ }^{\star}$ or (mass near/2 media) or (prevention near/5 (primary or secondary or universal or selective or target ${ }^{\star}$ or indicated))

\section{ICTRP search strategy:}

- Simple search - does not work as search engine strips away search sets

- Advanced search - form is setup to search in specific fields/elements

Condition field - Alcohol* or drink* or drunk* or intoxicat* or binge*

Intervention field - intervention ${ }^{\star}$ or educat* or promot* or adverti ${ }^{\star}$ or campaign ${ }^{\star}$ or (mass and media) or (prevention and (primary or secondary or universal or selective or target ${ }^{\star}$ or indicated))

\section{ClinicalTrials.gov search strategy - used Advanced Search screen. Truncation not permitted}

- (Alcohol OR alcohols OR alcoholic OR alcoholism OR drink OR drinks OR drunk OR drunks OR intoxicate OR intoxicated OR binge OR binges OR Binger)

- (Youth OR youths OR juvenile OR juveniles OR adolescence OR adolescent OR adolescents OR teen OR teenager OR teenagers OR schoolchild OR schoolchildren OR girl OR girls OR boy OR boys OR minor OR minors OR young OR early adult OR early adults)

\section{GOOGLE SCHOLAR search strategy - 260 character field length limit}

Searched for:

(alcohol* or drunk ${ }^{\star}$ or intoxicated ${ }^{\star}$ or binge ${ }^{\star}$ or drunk ${ }^{\star}$ ) and (youth ${ }^{\star}$ or juvenile* or adolescent ${ }^{\star}$ or teen* or school child* or girl* or boy $^{\star}$ or minor ${ }^{\star}$ or (young and (adult ${ }^{\star}$ or people ${ }^{\star}$ or person $\left.{ }^{\star}\right)$ ) or (early and adult ${ }^{\star}$ ) 


\section{Appendix 2. Criteria for risk of bias assessment}

\begin{tabular}{lll}
\hline Item & Judgement & Description \\
\hline $\begin{array}{l}\text { 1. Random se- } \\
\text { quence genera- } \\
\text { tion (selection } \\
\text { bias) }\end{array}$ & Low risk & $\begin{array}{l}\text { The investigators describe a random component in the sequence generation process } \\
\text { such as: random number table; computer random number generator; coin tossing; shuf- } \\
\text { fling cards or envelopes; throwing dice; drawing of lots; minimisation. }\end{array}$ \\
\cline { 2 - 3 } & High risk & $\begin{array}{l}\text { The investigators describe a non-random component in the sequence generation process } \\
\text { such as: odd or even date of birth; date (or day) of admission; hospital or clinic record } \\
\text { number; alternation; judgement of the clinician; results of a laboratory test or series of } \\
\text { tests; availability of the intervention. }\end{array}$ \\
\cline { 2 - 3 } & Unclear risk & $\begin{array}{l}\text { Insufficient information about the sequence generation process to permit judgement of } \\
\text { low or high risk. }\end{array}$ \\
\hline
\end{tabular}

2. Allocation con- Low risk
cealment (selec-
tion bias)

Investigators enrolling participants could not foresee assignment because 1 of the followtion bias) ing, or an equivalent method, was used to conceal allocation: central allocation (including telephone, web-based randomisation); sequentially numbered, opaque, sealed envelopes.

High risk Investigators enrolling participants could possibly foresee assignments because 1 of the following methods was used: open random allocation schedule (e.g. a list of random numbers); assignment envelopes without appropriate safeguards (e.g. envelopes were unsealed or nonopaque, envelopes were not sequentially numbered); alternation or rotation; date of birth; any other explicitly unconcealed procedure.

Unclear risk Insufficient information to permit judgement of low or high risk; this is usually the case if the method of concealment is not described or is not described in sufficient detail to allow a definitive judgement.

\begin{tabular}{lll}
$\begin{array}{l}\text { 3. Blinding of } \\
\text { participants and } \\
\text { providers (perfor- } \\
\text { mance bias) }\end{array}$ & Low risk & $\begin{array}{l}\text { Blinding of participants and key study personnel ensured, and unlikely that blinding } \\
\text { could have been broken. }\end{array}$ \\
\cline { 2 - 3 } & High risk & $\begin{array}{l}\text { No blinding or incomplete blinding, and the outcome is likely to be influenced by lack of } \\
\text { blinding. }\end{array}$
\end{tabular}

Blinding of key study participants and personnel attempted, but likely that the blinding could have been broken, and the outcome is likely to be influenced by lack of blinding.

\begin{tabular}{lll}
\cline { 2 - 3 } $\begin{array}{l}\text { 4. Blinding of out- } \\
\text { come assessor } \\
\text { (detection bias) }\end{array}$ & Low risk & $\begin{array}{l}\text { Insufficient information to permit judgement of low or high risk. } \\
\text { broken. }\end{array}$ \\
\cline { 2 - 3 } & High risk & $\begin{array}{l}\text { No blinding of outcome assessment, and the outcome measurement is likely to be influ- } \\
\text { enced by lack of blinding. }\end{array}$ \\
& $\begin{array}{l}\text { Blinding of outcome assessment, but likely that the blinding could have been broken, and } \\
\text { the outcome measurement is likely to be influenced by lack of blinding. }\end{array}$ \\
\cline { 2 - 3 } & Unclear risk & Insufficient information to permit judgement of low or high risk. \\
\hline $\begin{array}{l}\text { 5. Incomplete out- } \\
\text { come data (attri- } \\
\text { tion bias) for all } \\
\text { outcomes except }\end{array}$ & Low risk & No missing outcome data. \\
& $\begin{array}{l}\text { Reasons for missing outcome data unlikely to be related to true outcome (for survival da- } \\
\text { ta, censoring unlikely to be introducing bias). }\end{array}$
\end{tabular}


(Continued)

retention in treatment or dropout
Missing outcome data balanced in numbers across intervention groups, with similar reasons for missing data across groups.

For dichotomous outcome data, the proportion of missing outcomes compared with observed event risk not enough to have a clinically relevant impact on the intervention effect estimate.

For continuous outcome data, plausible effect size (difference in means or standardised difference in means) among missing outcomes not enough to have a clinically relevant impact on observed effect size.

Missing data have been imputed using appropriate methods.

All randomised participants are reported/analysed in the group they were allocated to by randomisation irrespective of non-compliance and co-interventions (intention to treat).

High risk Reason for missing outcome data likely to be related to true outcome, with either imbalance in numbers or reasons for missing data across intervention groups.

For dichotomous outcome data, the proportion of missing outcomes compared with observed event risk enough to induce clinically relevant bias in intervention effect estimate.

For continuous outcome data, plausible effect size (difference in means or standardised difference in means) among missing outcomes enough to induce clinically relevant bias in observed effect size.

'As-treated' analysis done with substantial departure of the intervention received from that assigned at randomisation.

Unclear risk Insufficient information to permit judgement of low or high risk (e.g. number randomised not stated, no reasons for missing data provided; number of dropouts not reported for each group).

\section{Selective re- Low risk} porting (reporting bias)
The study protocol is available and all of the study's pre-specified (primary and secondary) outcomes that are of interest in the review have been reported in the prespecified way.

The study protocol is not available but it is clear that published reports include all expected outcomes, including those that were prespecified (convincing text of this nature may be uncommon).

High risk Not all of the study's prespecified primary outcomes have been reported.

One or more primary outcomes are reported using measurements, analysis methods, or subsets of the data (e.g. subscales) that were not prespecified.

One or more reported primary outcomes were not prespecified (unless clear justification for their reporting is provided, such as an unexpected adverse effect).

One or more outcomes of interest in the review were reported incompletely so that they cannot be entered in a meta-analysis.

The study report fails to include results for a key outcome that would be expected to have been reported for such a study.

Unclear risk Insufficient information to permit judgement of low or high risk.
No difference in important co-variates (e.g. gender, alcohol use) between study groups at baseline.

No risk of contamination of programme effects (e.g. randomisation at school level of geographically dispersed schools). 
(Continued)

High risk Baseline between study group imbalance in important co-variate/s such as gender or alcohol use.

Contamination of programme effects (e.g. clusters of students randomised to experimental or control programme within 1 school). mination.

\section{CONTRIBUTIONS OF AUTHORS}

CG led the review and contributed at each stage of review development and completion.

LW contributed to the design and methods of the review, screening of records for inclusion, and final report writing.

DF contributed to design and methods of the review, and to final report writing and interpretation of results.

AW contributed to data extraction from included studies, entry of data into RevMan, assessment of risk of bias, meta-analysis, GRADE and final report writing.

MK contributed to development of the review protocol, assessment of risk of bias of included studies, and final report writing.

$\mathrm{RH}$ contributed to the development of the review protocol and advised on assessment of risk of bias of included studies, meta-analysis, and GRADE. RH also assisted in final report writing.

JT, TS, and ES contributed to screening of studies, extraction of data from included studies, and assessment of risk of bias of included studies.

JW, SS, and JR contributed to the design and methods of the review, and to final report writing.

\section{DECLARATIONS OF INTEREST}

This work was conducted in partnership between researchers from the University of Newcastle (Australia), and Oxford Brookes University (UK).

DFs department has received funding from the alcohol industry for adapting and evaluating a family-based prevention programme - the ISFP. The adapted version was evaluated in large-scale randomised controlled trials in Wales, funded by the UK Medical Research Council, and in Poland, funded by the Polish National Bureau for Drug Prevention.

\section{SOURCES OF SUPPORT}

\section{Internal sources}

- University of Newcastle, Australia.

In kind, author time

- Oxford Brookes University, UK.

In kind, author time

- Australian Drug Foundation, Australia.

Financial support for review assistance

- Hunter New England Population Health, Australia.

Financial support for review assistance

\section{External sources}

- No sources of support supplied 


\section{DIFFERENCES BETWEEN PROTOCOLAND REVIEW}

Through the conduct of the review, several minor modifications were made to planned methodological processes.

The protocol stated that we would describe missing data and all forms of attrition for each included study in the 'Risk of bias' table, and we would discuss the extent to which missing data could impact the conclusions of the review. Also, it was stated that missing data would be treated according to whether data were 'missing at random' or 'not missing at random'. In relation to the former, the main option was to analyse available data and ignore missing data. In the review, we in fact included information about attrition in the characteristics tables and in most cases used data to which multiple imputation or other treatment of missing data had been applied.

The protocol also stated that we would assess risk of performance and detection bias separately for objective and subjective outcomes. All outcomes reported were in fact subjective (self-reported) measures, and as such no risk of bias assessments are reported in association with objective measures.

Also, the protocol stated that for data not missing at random (e.g. participants who do not experience positive outcomes failing to complete follow-up assessments), imputation will be used to generate replacement values. When imputing missing dichotomous data, we will assume that missing data are negative (e.g. the participant demonstrated high-risk behaviour). When imputing missing continuous data, we will use a 'last observation carried forward' approach. Some relevant studies may fail to provide summary data (e.g. standard deviations). When this occurs, we will, if possible, obtain these data by performing calculations outlined in the Cochrane Handbook for Systematic Reviews of Interventions (Higgins 2011). In the review, it was not necessary to impute missing data. Most included studies reported already have done so or have otherwise handled missing data. We minimised data manipulation to conversion of published data into standard form for analysis (SEM and CI).

\section{INDEX TERMS}

\section{Medical Subject Headings (MeSH)}

${ }^{\star}$ Family Health; *Family Therapy [methods]; *Program Evaluation; Alcohol Drinking [epidemiology] ["prevention \& control]; Prevalence; Randomized Controlled Trials as Topic

\section{MeSH check words}

Adolescent; Child; Child, Preschool; Humans 